NBS

Publi.

cations

NBSIR 80-1630

A பூ05 036983

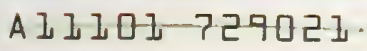

DETERMINATION OF MUTUAL COUPLING

BETWEEN CO-SITED MICROWAVE ANTENNAS

AND CALCULATION OF NEAR-ZONE ELECTRIC FIELD

C.F. Stubenrauch

A.D. Yaghjian

Electromagnetic Fields Division

National Engineering Laboratory

National Bureau of Standards

Boulder, Colorado 80303 



\section{DETERMINATION OF MUTUAL COUPLING BETWEEN CO-SITED MICROWAVE ANTENNAS AND CALCULATION OF NEAR-ZONE ELECTRIC FIELD}

C.F. Stubenrauch

A.D. Yaghjian

Electromagnetic Fields Division

National Engineering Laboratory

National Bureau of Standards

Boulder, Colorado 80303

June 1981

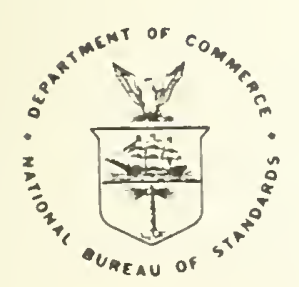

U.S. DEPARTMENT OF COMMERCE, Malcolm Baldrige, Secretary 

1. FURMULATION OF THE MUTUAL COUPLING BETWEEN TWO ANTENNAS......................

1.1 The Basic Coupling Formula (Transmission Integral)....................

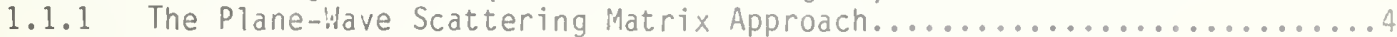

1.1.2 The Coupling Quotient in Terms of Far Field of Each Antenna........6

1.1.3 Coupling Quotient When the Roles of Transmitting and Receiving

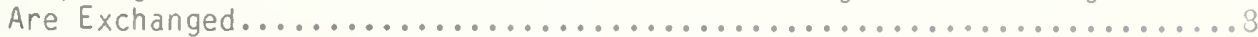

1.2 Eulerian Angle Transformations Describing the Arbitrary Orientation

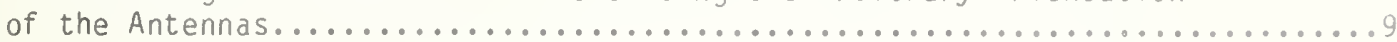

1.2.1 Rotational Transformations from $\left(k_{x}, k_{y}\right)$ to the Far-Field

Direction in the Fixed Coordinate System of Each Antenna............9

1.2.2 Vector Component Transformations Required to Compute the

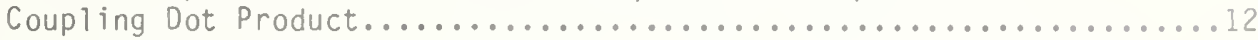

1.3 The Sampling Theorem, Limits of Integration, and Fast Fourier Transform.....15

1.3.1 The Point Spacing of $k_{x}$ and $k_{y}$ Required by the Sampling Theorem.....15

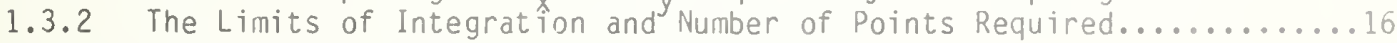

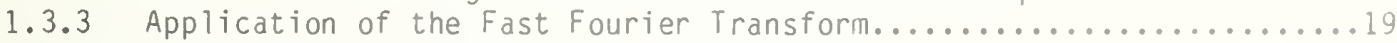

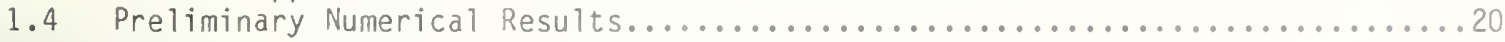

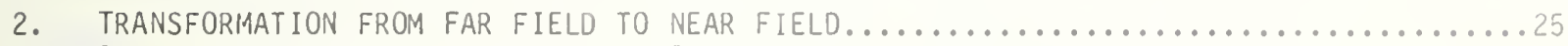

2.1 Relationship of Near-Field Intensities to Power Input and

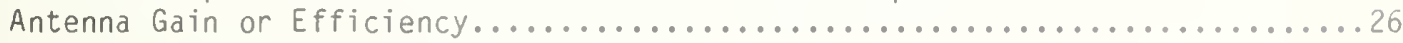

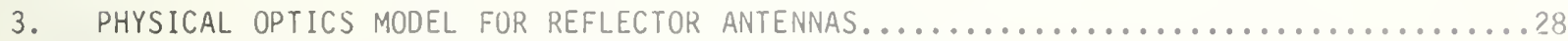

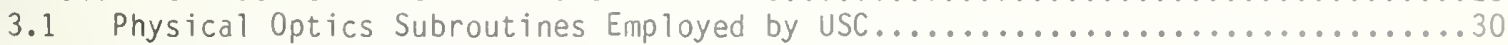

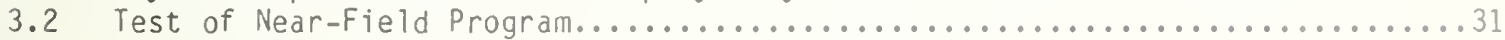

4. COMPARISON UF PHYSICAL UPTICS AND MEASURED FAR FIELDS.........................

5. COMPARISON OF PREDICTED AND MEASURED NEAR-FIELD COUPLING..................50

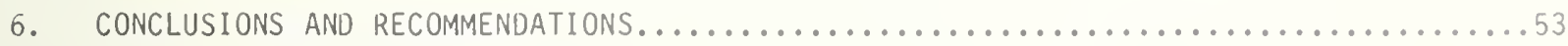

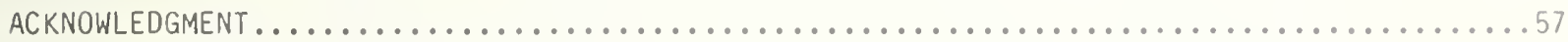

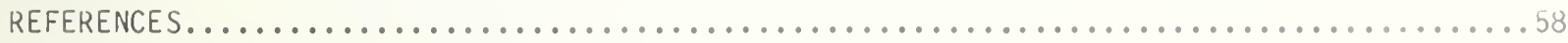

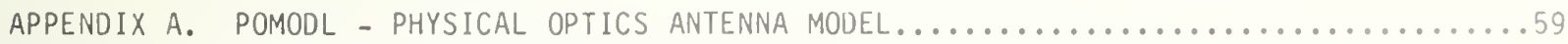

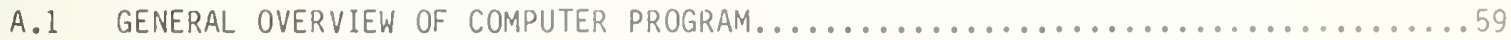

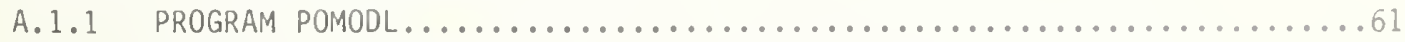

A.1.2 SUBROUTINE FAR2D(EPL, HPL, EY, NTHETA, NPHI, DATAX, IR $2 \times 2$, IC 2 TON) ........69

A.1.3 SUBROUTINE FFKXY (DATAY, NTHX2, NPHI, DATAX, IR $2 \times 2$, IC 2 TON $) \ldots \ldots \ldots \ldots \ldots 72$

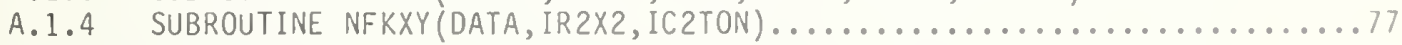

A.1.5 SUBROUTINE ETIOGAM(DATA $(1$, COL), NROW, NCOL, ICOL, ISGN, FLMDA, DELX,

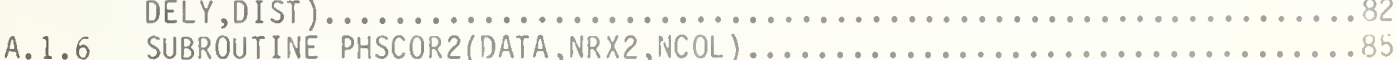

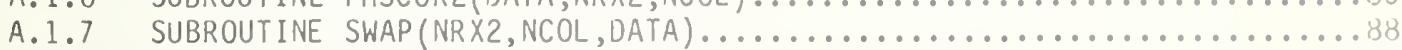

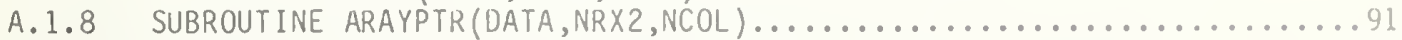

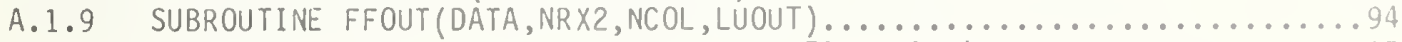

A.1.10 SUBROUTINE FOURT (DATA, NN, NDIIM, ISIGN, IFORIA, WORK) ..................

A.1.11 SUBROUTINE PARAB (FOD, DOL, BLOCK, DFOCUS, ACUSE, ACOSH, THETA, ETHETA,

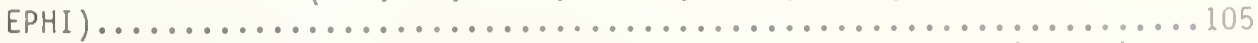

A.1.12 SUBROUTINE PLTI2OR(X,Y,XMAX, XIAIN, YMAX, YMIN, LAST, ISYMBOL, NO, MOST) .... I1.3

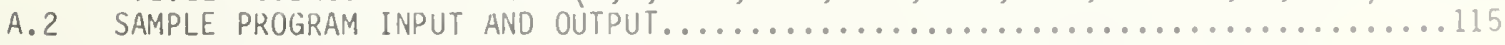

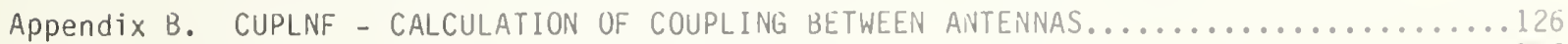

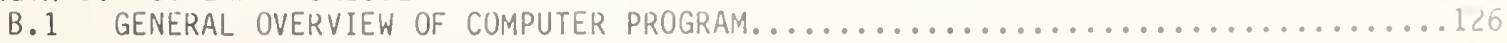




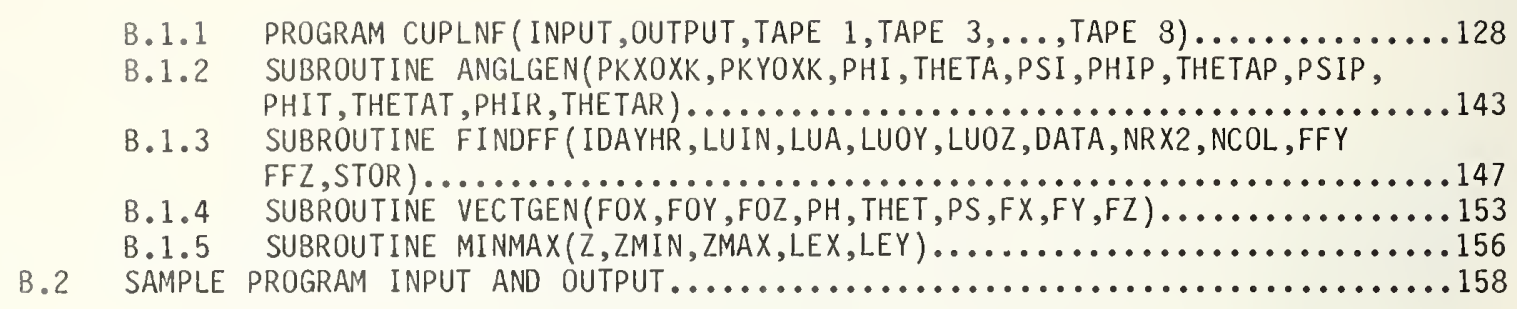

\section{LIST OF FIGURES}

Page

Figure 1. Coupling Schematic for two antennas $\left(0\right.$ and $0^{\prime}$ will be chosen at roughly the center of the radiating part of their respective antenna) $\ldots \ldots 5$

Figure 2. Definition of coordinates for the left antenna of figure $1 \ldots \ldots \ldots \ldots \ldots$

Figure 3. Definition of coordinate systems for the right antenna of figure $1 \ldots \ldots \ldots 13$

Figure 4. Physical interpretation for limits of integration. To a good approximation, only that portion of the spectrum within $\alpha$ is required to compute the

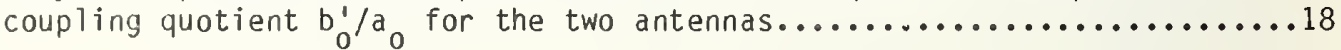

Figure 5. Hypothetical circular antennas directly facing each other

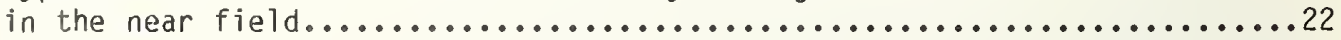

Figure 6. Coupling of circular antennas computed first using FFT integration, and then directly from far field along direction of separation..........23

Figure 7. Typical coupling curve for antennas skewed in their near field..........24

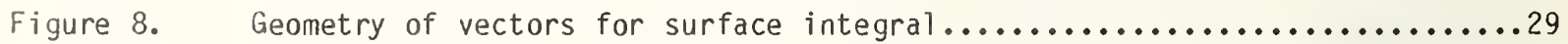

Figure 9a. Field strength in a uniformly illuminated aperture calculated using physical optics far fields. Dashed line indicates theoretical

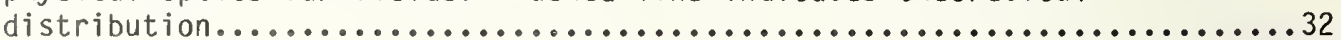

Figure 9b. Phase of field in a uniformly illuminated aperture calculated using

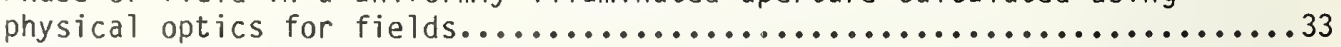

Figure 10a. Comparison of measured and calculated far-field patterns for antenna No. 1. E-plane cut, solid line - measured pattern, dashed line -

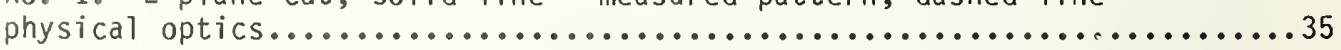

Figure 10b. Comparison of measured and calculated far-field patterns for antenna No. 1. H-plane cut, solid line - measured pattern, dashed line -

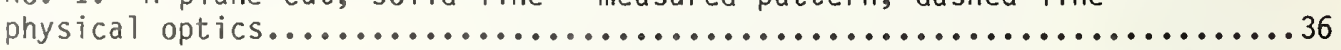

Figure 11a. Comparison of measured and calculated far-field patterns for antenna No. 2. E-plane cut, solid line - measured pattern, dashed line -

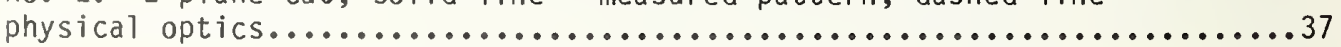

Figure 11b. Comparison of measured and calculated far-field patterns for antenna No. 2. H-plane cut, solid line - measured pattern, dashed line -

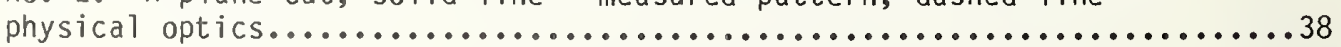


Figure 12a. Comparison of measured and calculated far-field patterns for antenna No. 3. E-plane cut, solid line - measured pattern, dashed line -

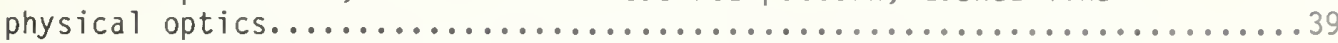

Figure 12b. Comparison of measured and calculated far-field patterns for antenna No. 3. H-plane cut, solid line - measured pattern, dashed line -

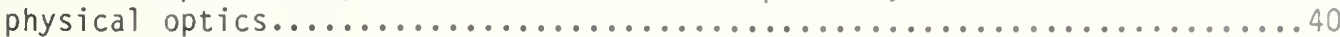

Figure 13. Comparison of measured and calculated far-field patterns for antenna No. 4. H-plane cut, solid line - measured pattern, dashed line -

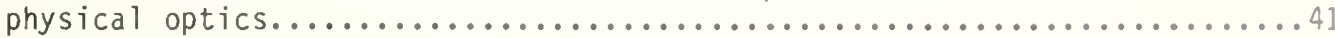

Figure 14. Comparison of effective current distribution used in physical optics and geometrical theory of diffraction calculations. (Uniform distribution assumed).

Figure 15. Diagram of multiple reflections involving feed structure............43

Figure 16a. Comparison of measured and calculated far-field patterns for antenna No. 1 with feed region covered with absorber. E-plane cut, solid curve measured pattern, dashed curve - physical optics...................44

Figure 16b. Comparison of measured and calculated far-field patterns for antenna No. 1 with feed region covered with absorber. H-plane cut, solid curve measured pattern, dashed curve - physical optics..................45

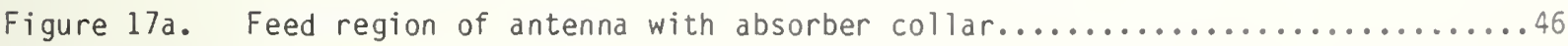

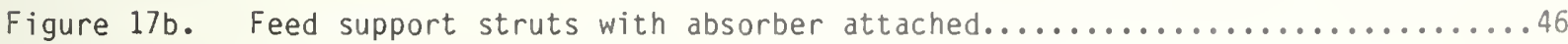

Figure 18a. Comparison of measured and calculated far-field patterns for antenna No. 1 with feed region covered with absorber. E-plane cut, solid curve measured pattern, dashed curve - physical optics..................47

Figure 18a. Comparison of measured and calculated far-field patterns for antenna No. 1 with feed region covered with absorber. H-plane cut, solid curve measured pattern, dashed curve - physical optics..................48

Figure 19. Photograph of experimental set up for measuring coupling between two reflector antennas...........................................

Figure 20. Schematic showing relative orientations of antennas for the

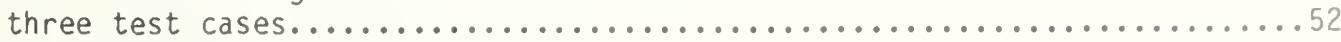

Figure 21. Mutual coupling between 1.2 meter reflector antennas.

Case 1: $\theta_{r}=0^{\circ}, \theta_{t}=0^{\circ}$. Solid lines indicate envelope of measured

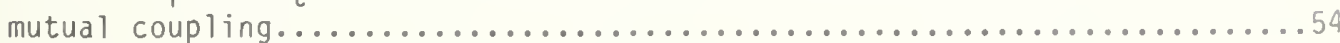

Figure 22. Mutual coupling between 1.2 meter reflector antennas.

Case 2: $\theta_{r}=15^{\circ}, \theta_{t}=0^{\circ}$. Solid lines indicate envelope of measured

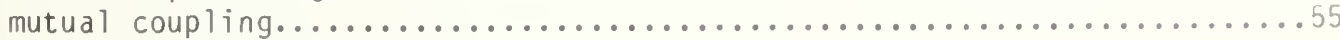

Figure 23. Mutual coupling between 1.2 meter reflector antennas.

Case 3: $\theta_{r}=15^{\circ}, \theta_{t}=20^{\circ}$. Solid lines indicate envelope of measured

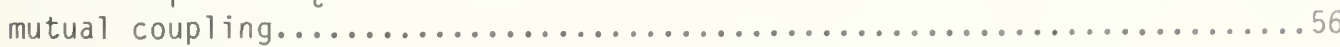





\section{DETERMINATION OF MUTUAL COUPLING BETWEEN CO-SITED MICROWAVE ANTENNAS AND CALCULATION OF NEAR-ZONE ELECTRIC FIELD}

\section{By}

C. F. Stubenrauch and A. D. Yaghjian

The theory and computer programs which allow the efficient computation of coupling between co-sited antennas given their far-field patterns are developed. Coupling between two paraboloidal reflector antennas is computed using both measured far-field patterns and far-field patterns which were obtained from a physical optics (PO) model. These computed results are then compared to the coupling measured directly on an outdoor antenna range. Far fields calculated using the $\mathrm{PO}$ model are compared to those obtained from transformed near-field measurements for several reflector antennas. Theory and algorithms are also developed for calculating near-field patterns from far fields obtained from the P0 model. Documentation of the near-field and coupling computer programs is presented in the appendices. Conclusions and recommendations for future work are included.

Key words: Co-sited antennas; coupling; far fields; near fields; physical optics; plane-wave spectrum; reflector antennas.

\section{INTRODUCTION}

This report discusses work done at the National Bureau of Standards (NBS) concerning problems related to the prediction of mutual coupling between antennas and the prediction of antenna near fields. In addition, comparisons for several paraboloidal reflector antennas were made between far-field patterns obtained from near-field measurements and those which were predicted using a physical optics (PO) model for the antennas.

A consequence of the scattering matrix theory of antennas and antenna-antenna interactions developed at NBS over the past 20 years [1] is that mutual coupling and near fields can be calculated provided the plane-wave spectra for the antenna or antennas are known. The essential, propagating part of a spectrum is related by a simple expression to the antenna's far-field pattern which may be determined, e.g., through model computation, direct far-field measurements, or transformed near-field measurements. For engineering studies of co-sited coupling or antenna near fields, expressing the quantities of interest in terms of the far fields proves especially convenient. In many cases the measured patterns are unavilable. Because it is possible to predict these patterns by employing a suitable model, part of the work described herein discusses the capability of a particularly convenient and efficient model: the physical optics computer program obtained from the University of Southern California (USC). 
Formulations of the mutual coupling problem in terms of antenna far fields are well known [7]; however, calculations using frevious theories have been deficient because of the large amounts of computation time and data required. In this work, it is shown that the functions to be integrated can be made band-limited; and thus the sampling theorem can be employed to determine the required point spacing, rather than the more usual trial-and-error method of testing convergence. Further, it is shown that the evaluation of mutual coupling requires only the far fields lying within the mutually subtended angles of the antennas. As a result of these improvements in the theory, an efficient program for calculating mutual coupling was written.

Section 1 of this report details the theory which allows rapid calculation of the mutual coupling between two antennas without restrictions on the separation distances. Section 2 discusses the specific problem of obtaining the near fields of an antenna given the far-field pattern. The PO model for reflector antennas is briefly discussed in section 3 as is the particular model employed. Far-field patterns which were predicted by the PO model and far-field patterns obtained from near-field measurements of actual antennas are compared in section 4. In section 5 coupling values measured directly in the laboratory are compared to those predicted from the theory of section 1 employing both modeled and measured far-fields. Conclusions and recommendations are given in section 6 .

The appendices describe the computer programs which perform the coupling and near-field calculations. Appendix A discusses and documents POMODL, a program which uses a PO model to calculate the far-field pattern for a reflector antenna and which calculates from this pattern the near-field distribution on a specified plane. The predicted far field also provides output for use as input by the program CUPLNF (described in sec. 1 and documented in Appendix B) which calculates the mutual coupling between two arbitrarily located and oriented antennas from their far-field patterns. 
The plane-wave scattering matrix (PWSM) description of antennas, introduced by Kerns at the NBS, forms an ideal theoretical framework on which to base the determination of mutual coupling between two collocated antennas. In fact, the basic PWSM formula required for the determination of the coupling between two antennas has existed for nearly twenty years [1]. However, before the existing formulas could be translated into a convenient program which computed coupling efficiently, three important tasks needed to be accomplished:

1) The Kerns coupling formula or transmission integral, as he calls it, was originally written in terms of the appropriate plane-wave spectrum for each antenna. For our purposes, we wanted to express the near-field mutual coupling in terms of the far field of each antenna (assuming reciprocal antennas) because usually the far field most conveniently characterizes an antenna and is most efficiently computed from, e.g., a PO-GTD (physica) optics and/or geometrical theory of diffraction) program or from near-field measurements. This task, although straightforward, requires careful attention to the details of definition of the far field, the plane-wave spectrum, and the reciprocity for each antenna.

2) The far fields of each antenna are usually expressed in a Cartesian coordinate system fixed in each antenna. To compute coupling for an arbitrary separation and orientation of two antennas, the coupling formula requires an integration of the dot product of the two vector far-field patterns in reoriented coordinate systems. Thus, task two consisted of expressing the reoriented coordinates of each antenna in terms of the Eulerian angles from the preferred or fixed coordinates in which the far field of the antenna was given. In addition, a similar transformation had to be applied in order to compute the dot product of the two vector far-field patterns. Again this task was fairly straightforward, yet rather tedious.

3) Finally, even though tasks (1) and (2) above recast the coupling or transmission integral in terms of the far fields of each antenna expressed in the preferred coordinate system fixed in each antenna, repeated evaluation of the double integrals (actually a double fourier transform) would require a prohibitive amount of computer time for electrically large microwave antennas unless the sampling theorem and FFT (fast Fourier transform) algorithm could be applied effectively. However, the application of the sampling theorem to these double Fourier transforms requires a sample spacing which, in general, is so small that repeated evaluation even by means of the FFT still becomes prohibitive. Moreover, the required sample spacing becomes smalier with increasing separation distance between antennas. Thus, the third major task was to discover a way to reduce drastically the computer time needed to evaluate the final form of the double integrals expressing the mutual coupling between two antennas.

The details of these three tasks and their accomplishment are described in the following three major sections $(1.1,1.2,1.3)$. 


\subsection{The Basic Coupling Formula (Transmission Integra1)}

This section begins with the transmission integral derived by Kerns [1] for the coupling of two antennas (when multiple reflections are neglected) in terms of the transmitting and receiving spectra of the respective antennas. The receiving antenna is assumed reciprocal, and its receiving spectrum is written in terms of its transmitting spectrum through the reciprocity relations. The transmitting spectrum of each antenna is then expressed in terms of the antenna's far electric field, which in turn yields a transmission integral or coupling formula in terms of the dot product of the vector far fields of each antenna. Finally, reciprocity is invoked for both antennas to prove that the mutual coupling is essentialiy the same when the roles of transmission and reception are exchanged.

\subsubsection{The Plane-Wave Scattering Matrix Approach}

Consider an arbitrary antenna transmitting with $e^{-i \omega t}$ time dependence to the left of an arbitrary receiving antenna, as shown in figure 1. The antennas may have arbitrary separation and orientation. Assume that only one mode propagates in the waveguide feed to each antenna. ${ }^{1}$ The incident waveguide mode coefficients for the left antenna are labeled $a_{0}$ and $b_{0}$ respectively, and for the right antenna, $a_{0}^{\prime}$ and $b_{0}^{\prime}$ respectively. The reflection coefficients of the right (receiving) antenna and its passive termination are denoted by $\Gamma_{0}^{\prime}$ and $\Gamma_{L}^{\prime}$ respectively.

The quantity $b_{0}^{1} / a_{0}$, which we shall call the coupling quotient, is a measure of how much signal couples into the receiving antenna per unit input into the transmitting antenna. If the same type of waveguide feeds each antenna and the receiving waveguide is terminated in a perfectly matched 1oad, $\left|b_{0}^{1} / a_{0}\right|^{2}$ equals the amount of power coupled to the receiving antenna per unit power incident to the transmitting antenna. (This power ratio expressed in decibels is commonly referred to as the insertion loss ratio.) Thus, $b_{0}^{1} / a_{0}$ is indeed the major parameter of interest in determining mutual interference between antennas.

The transmission integral which gives the coupling quotient in terms of transmitting and receiving plane-wave spectra of the respective antennas can be found directly from Kerns [1b]:

$$
\frac{b_{0}^{\prime}}{a_{0}}=\frac{1}{1-\Gamma_{L}^{\prime} \Gamma_{0}^{\top}} \int_{-\infty}^{\infty} \int_{-\infty}^{\infty} \underline{s}_{02}^{\prime}(\underline{K}) \cdot \underline{s}_{10}(\underline{K}) e^{i \gamma d} d \underline{K},
$$

where $\underline{s}_{10}(\underline{K})$ and $\underline{s}_{02}^{\prime}(\underline{K})$ are the "complete" transmitting and receiving spectra defined with respect to plane waves traveling in the common $\underline{k}$ direction but with phase reference to the

\footnotetext{
If more than one mode propagates in one or both of the feeds, this analysis can be applied for each possible transmit-receive pair of modes; and thus the analysis can be applied to "out-of-band" coupling.
} 


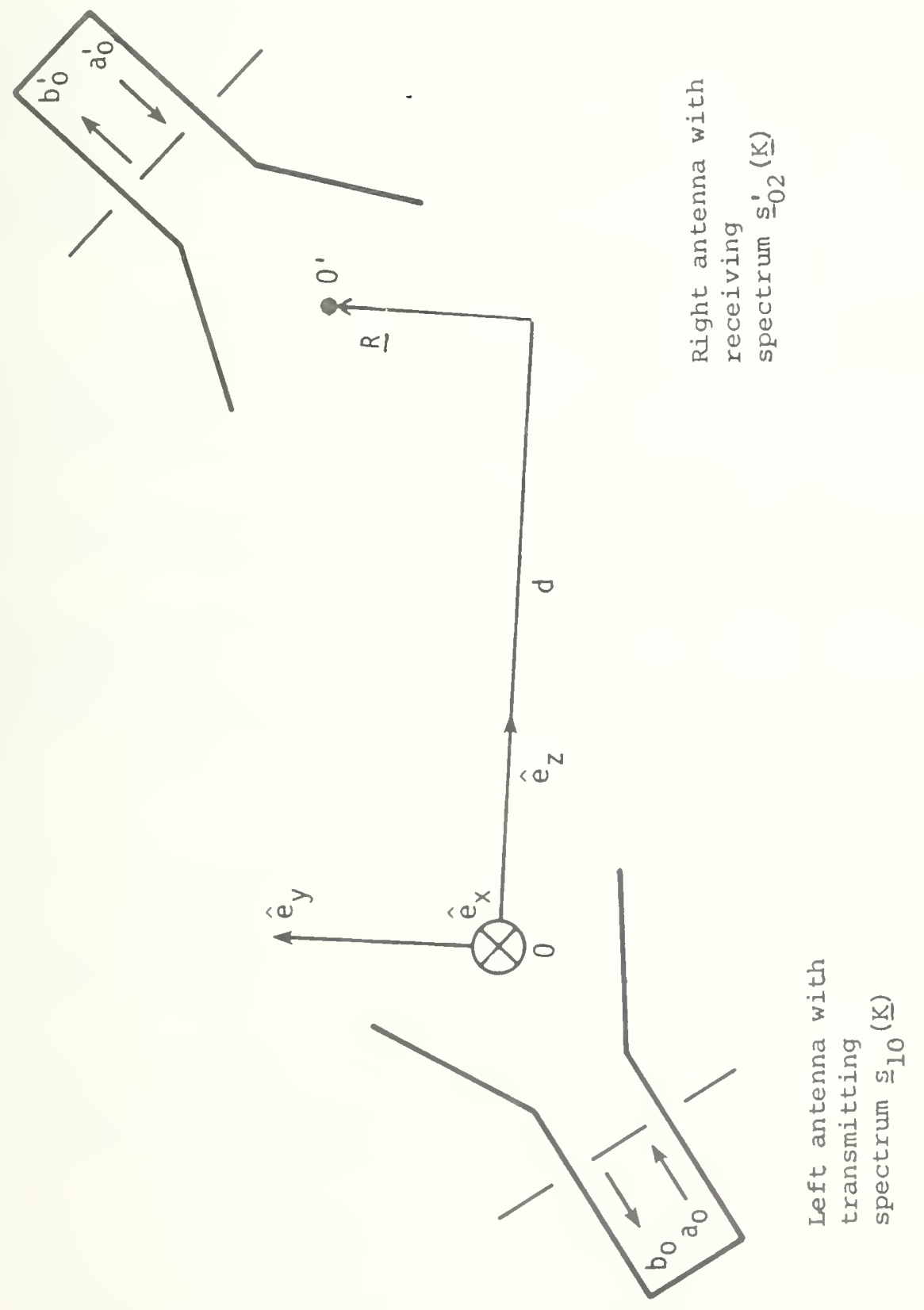

Figure 1. Coupling Schematic for two antennas 10 and $0^{\prime}$ will be chosen at roughly the center of the radiating part of their respective antenna). 
origins 0 and $0^{\prime}$ of the left (transmitting) and right (receiving) antennas respectively. The $z$ axis is chosen to run from 0 to $0^{\prime}$, with the distance $d=00^{\prime}$ and the $x-y$ axes perpendicular to the $z$ axis at 0 (see fig. 1). $\underline{k}=k_{x} \hat{e}_{x}+k_{y} \hat{e}_{y}$ is the transverse part of the propagation vector $\underline{k}=\underline{k}+\gamma \hat{e}_{z}\left(k=\frac{2 \pi}{\lambda}\right.$, where $\lambda$ is the wavelength $)$, and $\gamma=\left(k^{2}-k^{2}\right)^{1 / 2}$ is taken positive real for $k<k$ and positive imaginary for $k>k$. dK is shorthand notation for the double differential $\mathrm{dk}_{\mathrm{x}} \mathrm{dk} \mathrm{k}_{\mathrm{y}}$.

Equation (1) is an exact result from Maxwell's equation for two linear antennas operating with $e^{-i \omega t}$ time dependence in free space, when multiple reflections between the antennas are neglected. (In other words, the $b_{0}^{1} / a_{0}$ computed from eq (1) neglects power which enters the receiver after having been reflected from receiving antenna to transmitting antenna and back one or more times.) No other restrictive assumptions are involved. For example, the antennas may be lossy or even nonreciprocal.

Of course, eq (1) cannot be used to evaluate $b_{0}^{\prime} / a_{0}$ unless the spectra $\underline{s}_{02}^{\prime}$ and $\underline{s}_{10}$ are determined explicitly in terms of commonly measured or computed characteristics of the antenna. Toward this end, both spectra and eq (1) are recast in the next subsection in terms of the far electric fields of the antennas.

\subsubsection{The Coupling Quotient in Terms of Far Field of Each Antenna}

As a preliminary to expressing eq (1) in terms of the far fields of the antennas, assume that the receiving antenna contains no nonreciprocal devices or material so that its receiving functions $\underline{s}_{02}^{\prime}$ are related to $i$ ts transmitting functions $\underline{s}_{20}^{\prime}$ by the simple reciprocity formula [1b],

$$
\eta_{0}^{\prime} \underline{s}_{02}^{\prime}(\underline{K})=\frac{\gamma}{k Z_{0}} s_{20}^{\prime}(-\underline{K})
$$

A11 quantities in eq (2) have been defined in the previous section except the impedance of free space $z_{0}$ and $\eta_{0}^{\prime}$, which is the characteristic admittance of the propagated mode in the feed waveguide of the right (receiving) antenna of figure 1.

Substitution of $\underline{s}_{02}^{\prime}$ from eq (2) into eq (1) gives,

$$
\frac{b_{0}^{\prime}}{a_{0}}=\frac{\left(1-\Gamma_{L^{\prime}}^{\prime} \Gamma_{0}^{\prime}\right)^{-1}}{k Z_{0} \eta_{0}^{\prime}} \int_{K<k} \gamma \underline{s}_{20}^{\prime}(-\underline{K}) \cdot \underline{s}_{10}(\underline{K}) e^{i j d} d \underline{K} .
$$

Note that the integration limits in eq (3) have been made finite by eliminating the integration over the evanescent part of the spectra (included in the original infinite limits of eq (1)), thereby leaving only the radiating part of the spectra. This is permissible for all nonsuper-reactive antennas which are separated by a distance greater than a wavelength or so, i.e., if the antennas are outside each other's reactive field zone [2]; 
and if the contribution from the integration in eq (3) near the critical point $k=k$ is negligible, as is usually the case.

A major advantage of the PWSM techniques is that the radiating part of the spectrum of an antenna is proportional to the vector far field $\underline{E}(\underline{r})_{r \rightarrow \infty}$ of the antenna. Specifically, if $\underline{f}(\underline{r})$ refers to the normalized, complex far-electric-field pattern of the left (transmitting) antenna of figure 1 , i.e.,

$$
\underline{f}(\underline{r}) \equiv \frac{r e^{-j k r}}{a_{0}} \underline{E}(\underline{r})_{r \rightarrow \infty},
$$

then the radiating spectrum, $\underline{s}_{10}(\underline{k}), k<k$, is related to the complex far-field pattern by the disarmingly simple proportionality [1b],

$$
\underline{s}_{10}(\underline{k})=\frac{i}{\gamma} \underline{f}(\underline{k})
$$

Although $\underline{f}$ is shown as a function of $\underline{r}$ in eq (4), we know that the complex far-field pattern is a function only of the direction of $\underline{r}$; and thus $\underline{f}(\underline{k})$ in eq (5) is also only a function of the direction of $\underline{k}$ which is determined solely by the relative size of $k_{x}$ and $k_{y}$, the integration variables of eq (3).

Similarly, the radiating spectrum, $\underline{s}_{20}^{1}, k<k$, for the right (receiving) antenna in figure 1 can be written in terms of the normalized, complex, far-electric-field pattern $\underline{f}^{\prime}$ of that antenna:

$$
\underline{s}_{20}^{\prime}(-\underline{k})=\frac{i}{\gamma} \underline{f}^{\prime}(-\underline{k})
$$

where, as in eq (4), $\underline{f}^{\prime}$ is defined in terms of the far-electric-field $\underline{E}^{\prime}(\underline{r})_{r \rightarrow \infty}$ of the right antenna when it is radiating:

$$
\underline{f}^{\prime}(\underline{r}) \equiv \frac{r e^{-j k r}}{a_{0}^{\prime}} \underline{E}^{\prime}(\underline{r})_{r-\infty} .
$$

Substitution of the spectra from eqs (6) and (7) into eq (3) produces the coupling quotient for two antennas as a double integral over the dot product of the complex farelectric-field patterns of the antennas:

$$
\frac{b_{0}^{\prime}}{a_{0}}=-C^{\prime} \iint_{k<k} \frac{\underline{f}^{\prime}(-\underline{k}) \cdot \underline{f}(\underline{k})}{\gamma} e^{i \gamma d} d \underline{k} .
$$

In eq (8), $C^{\prime}$ is a consolidated notation for the "mismatch factor" $\left(1-\Gamma_{L}^{1} \Gamma_{0}^{1}\right)^{-1} / k Z_{0} \eta_{0}^{\prime}$. 


\subsubsection{Coupling Quotient When the Roles of Transmitting and Receiving are Exchanged}

The coupling quotient $b_{0}^{1 / a_{0}}$ in eq (8) is a measure of the signal which is received by the passively terminated antenna on the right side of figure 1 when an input mode of unit amplitude is applied to the transmitting antenna on the left. A natural and important question is what will be the coupling to the left antenna when the right antenna transmits at the same frequency and the left antenna is terminated in a passive load. Specificaliy, what is the expression for $b_{0} / a_{0}^{1}$ and how is it related to $b_{0}^{1} / a_{0}$ of eq (8).

The answer to this question can be obtained immediately by retracing the steps in the derivation of eq (8) but with the left antenna in figure 1 receiving and the right antenna transmitting. So doing, yields an expression for $b_{0} / a_{0}^{\prime}$ very similar to eq (8).

$$
\frac{b_{0}}{a_{0}^{\prime}}=-C \int_{K^{\prime}<k} \int_{\gamma^{\prime}} \frac{\underline{f}\left(-\underline{k}^{\prime}\right) \cdot \underline{f}^{\prime}\left(\underline{k}^{\prime}\right)}{\gamma^{\prime}} e^{i \gamma^{\prime} d} d \underline{k}^{\prime},
$$

where the "mismatch factor" $C$ is defined as before,

$$
C=\left(1-\Gamma_{L} \Gamma_{0}\right)^{-1} / k Z_{0} n_{0}
$$

$\Gamma_{0}$ and $\Gamma_{L}$ are now the reflection coefficients to the antenna on the left and its passive termination, respectively. And $n_{0}$ is now the characteristic admittance of the propagated mode in the waveguide feed to the left antenna. Because $\hat{e}_{z^{\prime}}=-\hat{e}_{z}$, we can choose $\hat{e}_{y^{\prime}}=\hat{e}_{y}$ and $\hat{e}_{x^{\prime}}=-\hat{e}_{x^{\prime}}$. Then changing the dummy integration variables in eq (9) from $k_{x}^{\prime}$ and $k_{y}^{\prime}$ to $k_{x}$ and $-k_{y}$ shows that the integration in eq (9) is identical to eq (8), i.e.,

$$
\frac{b_{0}}{a_{0}^{\prime}}=-c \int_{K<k} \int_{\frac{f}{(k}(\underline{k}) \cdot \underline{f^{\prime}(-\underline{k})}}^{\gamma} e^{i \gamma d} d \underline{k} .
$$

Comparing eqs (8) and (17), we see that the two coupling quotients, $b_{0}^{1} / a_{0}$ and $b_{0} / a_{0}^{\prime}$, are related merely through a constant factor, i.e.

$$
c^{\prime} \frac{b_{0}}{a_{0}^{1}}=c \frac{b_{0}^{\prime}}{a_{0}} \text {. }
$$

This means that if the coupling between two antennas is measured or computed with one of the antennas transmitting and the other receiving, the coupling, when the roles of transmitting and receiving are reversed, is also known (through ea (12)). A separate measurement or computation need not be done. Use of ea (12), of course, requires knowledae of the reflection coefficients and input admittances of each antenna contained in the definitions of $C$ and $C^{\prime}$. 
As a check, eq (12) was also derived directly from the "system two-port" equations describing the two antennas, by applying the Lorentz reciprocity theorem [1b] and knowing that multiple reflections between the antennas are being neglected. It can be further proven that if scattered fields are also negligibly received by the transmitting antenna, then the available power at the receiving antenna per unit input power to the transmitting antenna is the same when the rules of receiving and transmitting are reversed.

\subsection{Eulerian Angle Transformations Describing the Arbitrary Orientation of the Antennas}

From a quick look at eq (8), it might be concluded that the analysis required to compute the coupling between two antennas is essentially finished. All we need to do is compute or measure the vector far-field patterns of each antenna, take their dot product, and perform the double integration on a computer.

Unfortunately, a major problem, ignored so far, is the fact that the far-field pattern of an antenna is given with respect to a Cartesian coordinate system which is fixed in the antenna and which is not, in general, aligned with the Cartesian system shown in figure 1 to which the far-field patterns $\underline{f}(\underline{k})$ and $\underline{f}^{\prime}(-\underline{k})$ in eq $(8)$ are referenced. Thus, to use eq (8), it is mandatory that the far-field direction in the coordinate system fixed in each antenna corresponding to a given $\left(k_{x}, k_{y}\right)$ in eq (8) be determined explicitly. Moreover, to evaluate the dot product $\underline{f}^{\prime} \cdot \underline{f}$, the rectangular components of $\underline{f}$ and $\underline{f}^{\prime}$ in the $x-y-z$ system of figure 1 must be expressed in terms of the rectangular components of the coordinate systems fixed in the antennas.

Fortunately, all these necessary transformations can be accomplished by specifying the Eulerian angles required to align the axes fixed in each antenna with the $(x, y, z)$ axes chosen in figure 1 , as the following two subsections explain.

\subsubsection{Rotational Transformations from $\left(k_{x}, k_{y}\right)$ to the Far-Field Direction} in the Fixed Coordinate System of Each Antenna

Assume the left antenna in figure 1 has a fixed coordinate system with rectangular axes $\left(x_{A}, y_{A}, z_{A}\right.$ centered at 0$)$ in which the normalized far-electric-field pattern is given in terms of the spherical angles $\phi_{A}$ and $\theta_{A}$, as shown in figure $2 a$. That is, we have at our disposal, obtained from either measurement or computation, the vector far-field pattern $\underline{f}\left(\phi_{A}, \theta_{A}\right)$ as a function of $\phi_{A}$. and $\theta_{A}$.

Let $(\phi, \theta, \psi)$ be the Eulerian angles needed to rotate the $\left(x_{A}, y_{A}, z_{A}\right)$ axes in line with the $(x, y, z)$ coupling axes of figure 1 . Specifically, as shown in figure 2b, rotate an angle $\phi(0 \leq \phi<2 \pi)$ about the positive $z_{A}$ axis, thereby changing the direction of $x_{A}$ and $y_{A}$ but not $z_{A}$. Then rotate an angle $\theta(0 \leq \theta \leq \pi)$ about the new positive $y_{A}$ axis, thereby changing the direction of $z_{A}\left(\right.$ to $z$ ) and again $x_{A}$ but not $y_{A}$. ( $\phi$ and $\theta$ are the usual spherical angles.) Finally, rotate an angle $\psi(0 \leq \psi<2 \pi)$ about the positive $z$ axis to align the new $x_{A}$ and $y_{A}$ axes with $x$ and $y$. These are fairly common definitions of Eulerian angle rotations found in a number of textbooks such as reference [3]. 


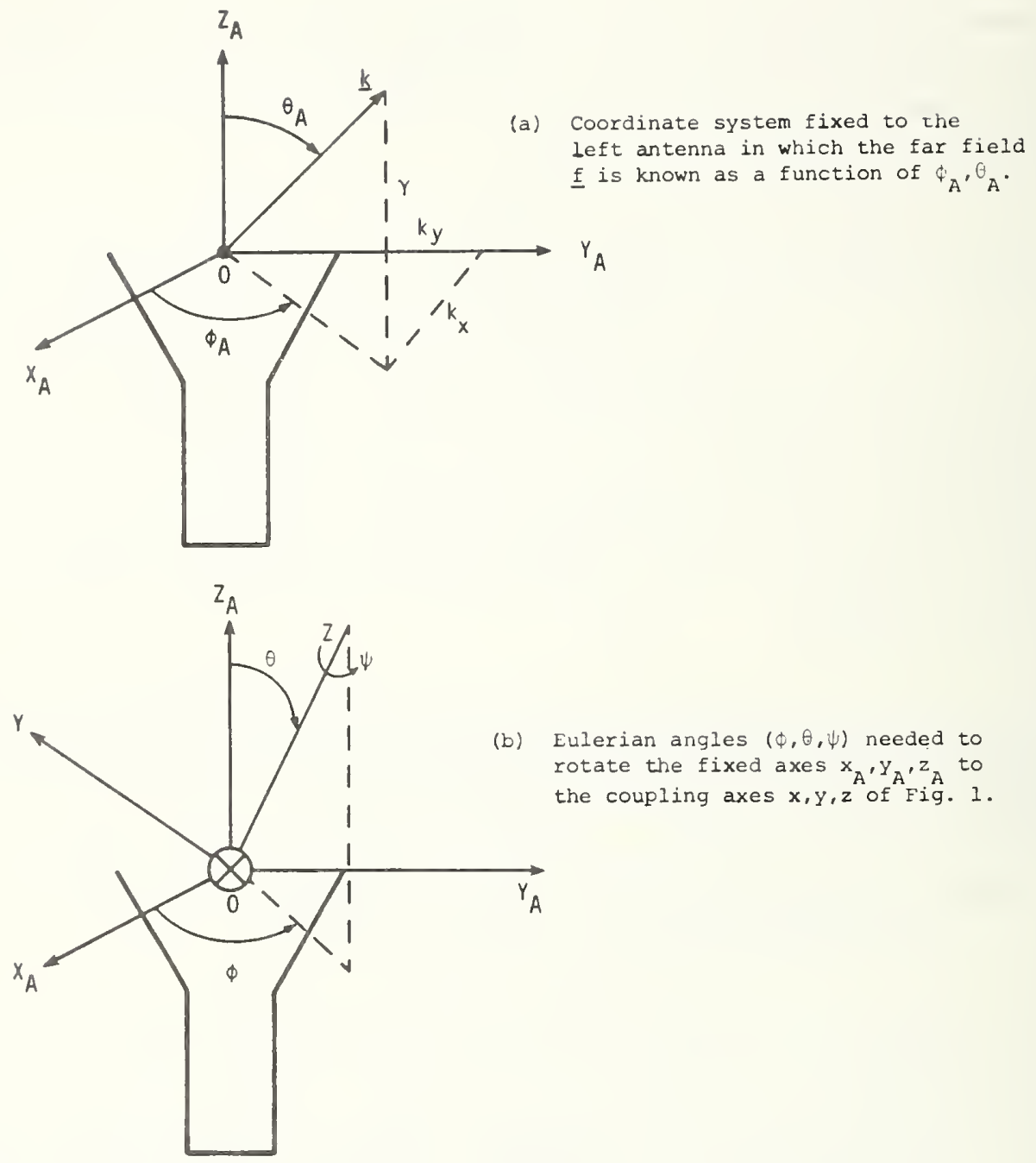

Figure 2. Definition of coordinates for the left antenna of figure 1. 
To understand the transformation needed to evaluate eq (8), note in eq (8) that $f$ and $f^{\prime}$ are written as functions of $\underline{k}_{=} k_{x} \hat{e}_{x}+k_{y} \hat{e}_{y}+\gamma \hat{e}_{z}$ or, in other words, as functions of $k_{x}$ and $k_{y}$ because $\gamma$ is determined from $k_{x}$ and $k_{y}$. However, we are given as known (measured or computed) $\bar{f}$ as a function of $\phi_{A}$ and $\theta_{A}$, not $k_{x}$ and $k_{y}$. Consequently, to evaluate eq (8) numerically, a transformation is needed which will convert $\left(k_{x}, k_{y}\right)$ to $\left(\phi_{A}, \theta_{A}\right)$ under the given Eulerian angles $(\phi, \theta, \psi)$ defining the $x_{A}-y_{A}-z_{A}$ system with respect to the $x-y-z$ system. This Eulerian transformation, which is a straightforward, rather lengthy, linear transformation found in a number of textbooks [3], wi11 not be derived here but simply stated in the form useful for our purposes of evaluating eq (8).

Before actually writing the required expression for $\phi_{A}$ and $\theta_{A}$, the antenna on the right side of figure 1 should also be discussed because it will require a similar transformation to convert $k_{x}$ and $k_{y}$ to the spherical angles of its preferred system. That is, if the far-field pattern $\underline{f}^{\prime}$ of this right antenna is known (measured or computed) in terms of spherical angles $\phi_{p}$ and $\theta_{p}$ with respect to $\left(x_{p}, y_{p}, z_{p}\right)$ axes fixed to the antenna (and centered at $\left.0^{\prime}\right)$, then $\left(\phi_{p}, \theta_{p}\right)$ are needed as functions of $\left(k_{x}, k_{y}\right)$ in order to evaluate $\underline{f}^{\prime}(-\underline{k})$ in eq (8) (see fig. 3). (An important point to remember is that $\underline{f}^{\prime}(-\underline{k})$ denotes the value of the far-field pattern in the -k direction.) ATso, as shown in figure 3 , let $\phi^{\prime}, \theta^{\prime}$, and $\psi^{\prime}$ denote the Eulerian angles which rotate the $\left(x_{p}, y_{p}, z_{p}\right)$ axes fixed in the right antenna parallel to the $((-x), y,(-z))$ coupling axes of figure 1 .

Both transformations, from $\left(k_{x}, k_{y}\right)$ to $\left(\phi_{A}, \theta_{A}\right)$ and $\left(\phi_{p}, \theta_{p}\right)$, are similar and can be written explicitly as:

$$
\cos \left(\begin{array}{l}
\theta_{A} \\
\theta_{p}
\end{array}\right)=-\sin \left(\begin{array}{l}
\theta^{\prime} \\
\theta^{\prime}
\end{array}\right) \cos \left(\begin{array}{l}
\psi^{\prime} \\
\psi^{\prime}
\end{array}\right) \frac{k_{x}}{k} \pm \sin \left(\begin{array}{l}
\theta^{\prime} \\
\theta^{\prime}
\end{array}\right) \sin \left(\begin{array}{l}
\psi \\
\psi^{\prime}
\end{array}\right) \frac{k_{y}}{k}+\cos \left(\begin{array}{l}
\theta^{\prime} \\
\theta^{\prime}
\end{array}\right) \frac{\gamma}{k}
$$

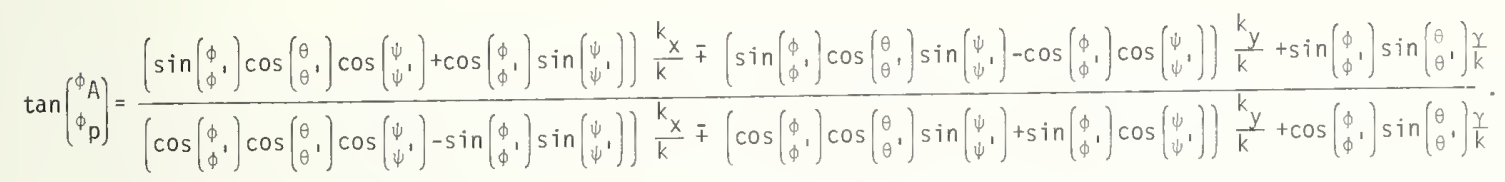

The top signs in eqs (13) go with $\left(\phi_{A}, \theta_{A}\right)$, the bottom with $\left(\phi_{p}, \theta_{p}\right)$. Equations (13) Took rather cumbersome at first sight, yet computationally they are quite manageable because they involve only sines and cosines of the Eulerian angles and linear dependence upon $k_{x}, k_{y}$, and $\gamma$ (which equals $\left.\sqrt{k^{2}-\left(k_{x}^{2}+k_{y}^{2}\right)}\right)$. The computer program merely contains a subroutine which yields $\left(\phi_{A}, \theta_{A}\right)$ and $\left(\phi_{p}, \theta_{p}\right)$ from eqs (13) when given the Euterian angles $(\phi, \theta, \psi),\left(\phi^{\prime}, \theta^{\prime}, \psi^{\prime}\right)$, and $\left(k_{x}, k_{y}\right)$ as input.

With the transformations of eqs (13), eq (8) can now be expressed in terms of $\left(\theta_{A}, \phi_{A}\right)$ and $\left(\phi_{p}, \theta_{p}\right)$ : 


$$
\frac{b_{0}^{\prime}}{a_{0}}=-C^{\prime} \int_{K<k} \int_{k} \frac{f^{\prime}\left(\phi_{p}, \theta_{p}\right) \cdot \underline{f}\left(\phi_{A}, \theta_{A}\right)}{\gamma} e^{i \gamma d} d \underline{K} .
$$

\subsubsection{Vector Component Transformations Required to Compute the Coupling Dot Product}

In the previous subsection a transformation was written that yielded $f$ and $\underline{f}^{\prime}$ in eq (14) as functions of the spherical angles $\left(\phi_{A}, \theta_{A}\right)$ and $\left(\phi_{p}, \theta_{p}\right)$ in which the far-field patterns were measured or computed. Sti11, a method is needed to compute the dot product $\underline{f}^{\prime} \cdot \underline{f}$, because the components of $\underline{f}$ and $\underline{f}^{\prime}$ are given in terms of unit vectors of the $\left(x_{A}, y_{A}, z_{A}\right)$ and $\left(x_{p}, y_{p}, z_{p}\right)$ coordinate systems fixed respectively in the left and right antennas of figure 1. And these two sets of unit vectors have relative directions which depend also on the Eulerian angles $(\phi, \theta, \psi)$ and $\left(\phi^{\prime}, \theta^{\prime}, \psi^{\prime}\right)$.

A convenient way to evaluate $\underline{f}^{\prime} \cdot \underline{f}$ is to first write $\underline{f}$ and $\underline{f}^{\prime}$ in the $(x, y, z)$ and $\left(x^{\prime}, y^{\prime}, z^{\prime}\right)$ rectangular components respectively shown in figures 2 and 3 ,

$$
\begin{aligned}
& \underline{f}=f_{x} \hat{e}_{x}+f_{y} \hat{e}_{y}+f_{z} \hat{e}_{z} \\
& f^{\prime}=f_{x^{\prime}}^{\prime} \hat{e}_{x^{\prime}}+f_{y^{\prime}}^{\prime} \hat{e}_{y^{\prime}}+f_{z^{\prime}}^{\prime} \hat{e}_{z^{\prime}} .
\end{aligned}
$$

Because by definition,

$$
\hat{e}_{x^{\prime}}=-\hat{e}_{x^{\prime}}, \hat{e}_{y^{\prime}}=\hat{e}_{y^{\prime}} \text {, and } \hat{e}_{z^{\prime}}=-\hat{e}_{z^{\prime}} \text {, }
$$

the dot product becomes

$$
\underline{f}^{\prime} \cdot f=-f_{x^{\prime}}^{\prime} f_{x}+f_{y^{\prime}}^{\prime} f_{y}-f_{z^{\prime}}^{\prime} f_{z} .
$$

Next, we express the rectangular components of eq (17) in the rectangular components with respect to the fixed axes $\left(x_{A}, y_{A}, z_{A}\right)$ and $\left(x_{p}, y_{p}, z_{p}\right)$, again through the appropriate Eulerian transformation. In matrix notation

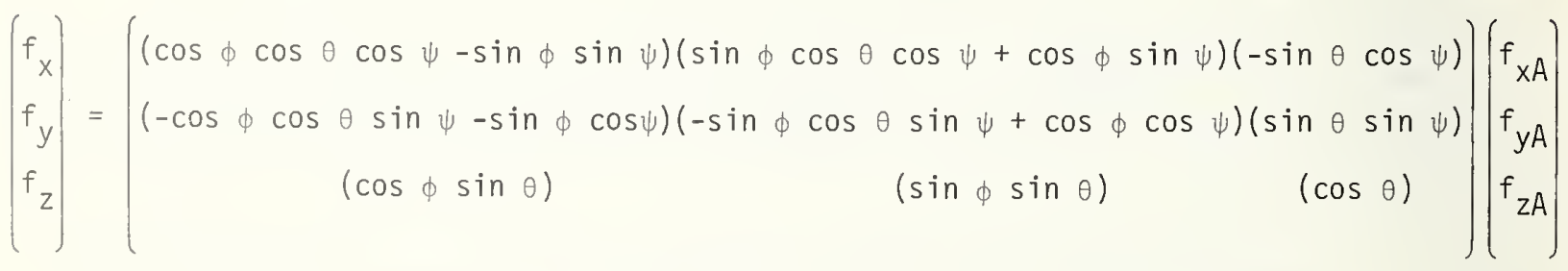




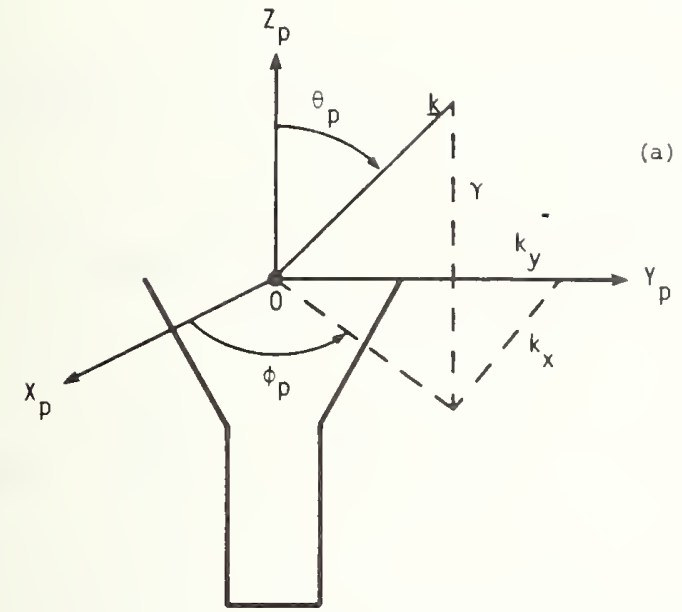

(a) Coordinate syster fixed to the right antenna in which the far field $\underline{f}^{\prime}$ is known as a function of $\psi_{p}, \theta_{p}$

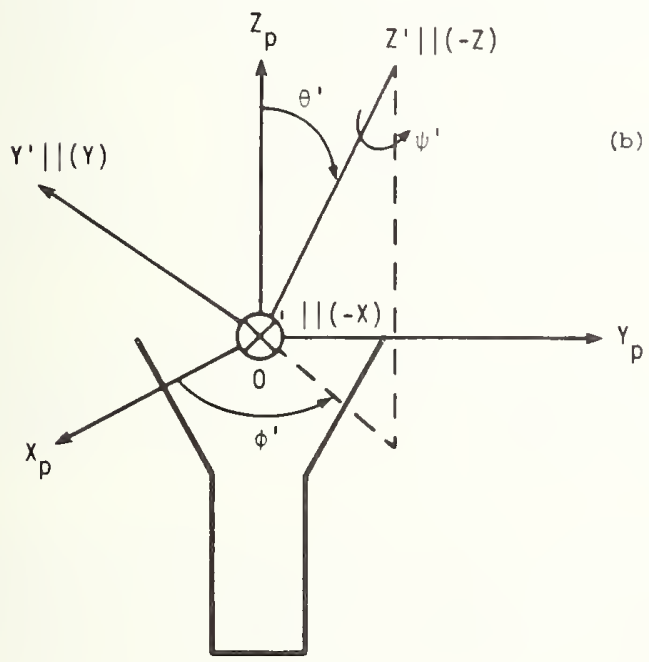

b) Eulerian angles ( $\left.\varphi^{\prime}, \theta^{\prime}, \psi^{\prime}\right)$ needed to rotate the fixed axes $x, y, z$ to the coupling axes $x^{\prime}, y^{\prime}, z^{\prime}$, which are in the direction of the $(-x),(y),(-z)$ axes of $\mathrm{Ei}$. 1.

Figure 3. Definition of coordinate systems for the right antenna of figure 1. 
The counterpart equation for $\left(f_{x^{\prime}}^{\prime}, f_{y^{\prime}}^{\prime}, f_{z^{\prime}}^{\prime}\right)$ is the same as eq (18) but with $\left(\phi^{\prime}, \theta^{\prime}, \psi^{\prime}\right)$ and $\left(f_{x p}^{\prime}, f_{y p}^{\prime}, f_{z p}^{\prime}\right)$ replacing $(\phi, \theta, \psi)$ and $\left(f_{x A}, f_{y A}, f_{z A}\right)$, respectively. It should also be noted that the $x, y$, and $z$ components of the far field are not independent because there is no radial component of far field. Using $f_{A}$, for an example, the rectangular components are related by $\cos \phi_{A} \sin \theta_{A} f_{x A}+\sin \phi_{A} \sin \theta_{A} f_{y A}+\cos \theta_{A} f_{z A}=0$.

If the far-field components $\left(f_{x A}, f_{y A}, f_{z A}\right)$ for the left antenna and $\left(f_{x p}^{\prime}, f_{y p}^{\prime}, f_{z p}^{\prime}\right)$ for the right antenna of figure 1 are known, eq (18) and its counterpart equation yield $\left(f_{x}, f_{y}, f_{z}\right)$ and $\left(f_{x^{\prime}}^{\prime}, f_{y^{\prime}}^{\prime}, f_{z^{\prime}}^{\prime}\right)$ in terms of the given Eulerian angles. In turn, eq (17) yields the dot product $\underline{f}^{\prime} \cdot \underline{f}$. Again, the computer program which computes the double integral (14) need only contain a simple subroutine to evaluate eq (18), and the dot product $\underline{f}^{\prime} \cdot f$ is immediately computable from eq (17).

One other set of transformations often proves useful, however. Usually, the far field of an antenna is given not in terms of rectangular components but in terms of spherical components. If the far-electric-field pattern of the left and right antennas of figure 1 are known in terms of $\left(f_{\theta A}, f_{\phi A}\right)$ and $\left(f_{\theta p}, f_{\phi p}\right)$ respectively, then the rectangular components are related to these spherical components by the spherical angles. Specifically,

$$
\left(\begin{array}{c}
f_{x A} \\
f_{y A} \\
f_{z A}
\end{array}\right)=\left(\begin{array}{ccc}
-\sin \phi_{A} & \cos \theta_{A} \cos \phi_{A} \\
\cos \phi_{A} & \cos \theta_{A} \sin \phi_{A} \\
0 & -\sin \theta_{A}
\end{array}\right)\left(\begin{array}{c}
f_{\phi A} \\
f_{\theta A}
\end{array}\right)
$$

The counterpart equation giving $\left(f_{x p}^{\prime}, f_{y p}^{\prime}, f_{z p}^{\prime}\right)$ as functions of $\left(f_{\phi p}^{\prime}, f_{\theta p}^{\prime}\right)$ is formed from eq (19) merely by replacing $\left(\phi_{A}, \theta_{A}\right)$ in the matrix with $\left(\phi_{p}, \theta_{p}\right)$.

In surmary, if $\left(f_{\phi A}, f_{\theta A}\right)$ and $\left(f_{\phi p}^{\prime}, f_{\theta p}^{\prime}\right)$ are the known far-electric-field patterns in the fixed coordinate systems of the left and right antennas of figure 1 , respectively, eq (19) and its counterpart transform these spherical components to rectangular components. Equation (18) and its counterpart transform these rectangular components in the fixed systems to rectangular components in the coupling $(x, y, z)$ or $\left(x^{\prime}, y^{\prime}, z^{\prime}\right)$ coordinates. Final1y, eq (17) yields the required dot product from the transformed components.

These transformations must be done for each $\left(k_{x}, k_{y}\right)$ within the limits of integration needed to evaluate eq (14). Moreover, eqs (13) must be evaluated for each $\left(k_{x}, k_{y}\right)$. Fortunately, the nature of the integrals in eq (14) allows the application of the sampling theorem and fast Fourier transform, as well as the limits of integration to be reduced inversely proportional to $d$. These topics, which enable the efficient computer evaluation of the mutual coupling quotient, are covered in the following section. 
1.3. The Sampling Theorem, Limits of Integration, and Fast Fourier Transform

This section shows how the sampling theorem converts the double integration in eq (14) to a double summation which can be summed using the fast Fourier transform (FFT) algorithm. In addition, the effective limits of integration are shown to reduce inversely proportional to $d$, the separation distance $00^{\prime}$ between the two antennas.

\subsubsection{The Point Spacing of $k_{x}$ and $k_{y}$ Required by the Sampling Theorem}

Equation (14) represents the coupling quotient for the two antennas positioned in figure 1. If the antenna on the right side of figure 1 is displaced by a vector $\underline{R}$ perpendicular to the $z$ axis, the integrand in eq (14) changes only by the phase factor $\exp (i \underline{k} \cdot \underline{R})=\exp \left(i k_{x} x+i k_{y} y\right)$. That is, eq (14) can be written more general1y as

$$
\frac{b_{0}^{\prime}(\underline{R}, d)}{a_{0}}=-C^{\prime} \iint_{K<k} \frac{f^{\prime}\left(\phi_{p}, \theta_{p}\right) \cdot \underline{f}\left(\phi_{A}, \theta_{A}\right)}{\gamma} e^{i \gamma d} e^{i \underline{K} \cdot \underline{R}} d \underline{K} .
$$

The sampling theorem [4] could be applied to convert the double Fourier transform in eq $(20)$ to a double Fourier series, if $b_{0}^{\prime}(\underline{R}, d)$ were zero outside a finite $|\underline{R}|=R_{0}$. Now $b_{0}^{\prime}(R, d)$ behaves as $1 / \sqrt{R^{2}+d^{2}}$ as $R \rightarrow \infty$, and thus, strictly speaking, will never vanish for finite $R_{0}$. However, if we choose $R_{0}>d, b_{0}^{\prime}$ is small and the "aliasing" error introduced by using the sampling theorem should be small, especially near $\underline{R}=0$, even though $b_{0}^{\prime}$ is not strictly "band limited" (i.e., zero outside a finite range).

In view of the decay of $b_{0}^{\prime}$ with $R$, choose

$$
R_{0}=B d,
$$

where $B$ is a number much greater than 1. (Computations show that in practice, a $B$ no larger than 1 or 2 is often sufficient for the accurate calculation of $b_{0}^{\prime}(\underline{R}, d)$ near $\underline{R}=0$ from eq (23) below. For larger $\underline{R}$, greater $B$ is generally required. Also, $R_{0}$ should never be smaller than about the sum of the diameters of the two antennas.) The sampling theorem applied to eq (20) then requires a sample spacing no larger than

$$
\frac{\Delta k_{x}}{k}, \frac{\Delta k_{y}}{k}=\frac{\lambda}{2 B d},
$$

in order to convert eq (20) to the double summation,

$$
\frac{b_{0}^{\prime}(\underline{R}, d)}{a_{0}}=-C^{\prime} \Delta k_{x} \Delta k_{y} \sum_{m=-M}^{M} \sum_{\ell=-L}^{L} \frac{f^{\prime}\left(\phi_{p}^{l m}, \theta_{p}^{l m}\right) \cdot \underline{f}\left(\phi_{A}^{l m}, \theta_{A}^{l m}\right)}{\gamma_{l m}} e^{i \gamma_{\ell m} d} e^{i \underline{k}_{\ell \dot{m}} \underline{R}},
$$

where

$$
\frac{\underline{k}_{\ell m}}{k}=\frac{\ell \lambda}{2 B d} \hat{e}_{x}+\frac{m \lambda}{2 B d} \hat{e}_{y}
$$


and $\ell, m$ are integers which range to cover the limits of integration $\left|\underline{k}_{\ell m}\right|<k$ (i.e., $\left.L, M \simeq \frac{2 B d}{\lambda}\right)$.

The beauty of eq (23) is not only that the integrals have been converted to summations, which can be performed on a computer, but also that the summation is ideally suited for evaluation by means of the FFT algorithm, which decreases the running time considerably when the coupling quotient over a range of $\underline{R}$ is desired.

\subsubsection{The Limits of Integration and Number of Points Required}

The number of points required to compute the double summation of eq (23) is approximately $(2 B d / \lambda)^{2}$ for each separation $(\underline{R}, d)$ and orientation of the antennas. For $d / \lambda$ of appreciable size, the number of points can become so large that the computer time required to evaluate eq (23) over a range of $\underline{R}$, even using the FFT, can become exorbitant. For example, if $d=10$ meters and $\lambda=3 \mathrm{~cm}$, choosing a typical value of $B=2$ yields $(2 B d / \lambda)^{2}=$ $1.8 \times 10^{6}$ terms to be summed for each separation and orientation of the antennas. Fortunately, however, it can be shown that the effactive limits of integration, i.e., $M$ and $L$ in eq (23), can be reduced inversely proportional to the separation distance $d$ to keep the total number of summation points bounded to a manageable number regardless of the value of the separation d between antennas.

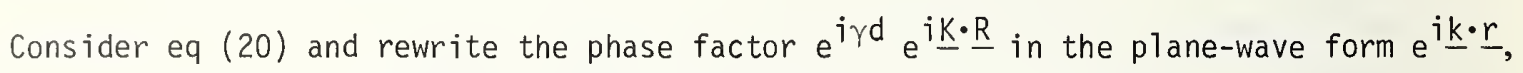
where $\underline{r}=\underline{R}+\mathrm{de}_{\mathrm{z}}$. For $r$ much larger than the dimension of either antenna, the function $e^{i k \cdot r}$ oscillates more rapidly than the oscillations of the far-field pattern dot product $\underline{f}^{\prime} \cdot \underline{f}$, except when $\underline{k}$ is in the directions approximately parallel to $\underline{r}$. This means that the integration in eq (20) will essentially cancel to zero except for the contribution near $\underline{k}$ equal to $r$, provided the contribution from near the critical point $k=k$ is negligible, as is usually the case. In particular, a more thorough analysis of the integration in eq (20) reveals that in order to compute the coupling quotient for values of $|\underline{R}|$ between 0 and $R$, only the part of the spectrum defined by

$$
\frac{K}{k}<\frac{R}{r}+\frac{\left(D+D^{\prime}\right)}{r}, \quad\left(r>R+D+D^{\prime}\right)
$$

contributes significantly to the integration (under the assumed provision of negligible contribution from the end critical point). The quantities $D$ and $D^{\prime}$ in the inequality (25) refer to the overall dimension of each of the antennas except when $D$ and/or $D$ ' is less than $2 \lambda$, in which case $D$ and/or $D^{\prime}$ is set equal to $2 \lambda .{ }^{2}$ For example, if each antenna were an electrically large, circular aperture type of radiator, $D$ and $D$ ' would be their respective diameters; but if one or the other of the antennas were a short dipole, its effective diameter would be set equal to $2 \lambda$. Of course, nearly all microwave antennas have dimensions much greater than $2 \lambda$.

\footnotetext{
Equation (25) assumes implicitly that the origins 0 and $0^{\prime}$ for the two antennas by which $r$
} is defined $\left(r=00^{\prime}\right)$ are chosen near the physical centers of their respective antennas. 
For $R \ll\left(D+D^{\prime}\right)$, i.e., coupling along the $z$ axis as shown in figure 1 , the criterion (25) reduces to simply $k / k<\frac{D+D^{\prime}}{d}$, and the limits of integration in eq (20) become

$$
k<\frac{k\left(D+D^{2}\right)}{d},\left(d>D+D^{\prime}>>R\right) .
$$

As d gets much larger than the sum of the overall dimensions of the two antennas, eq (26) shows that the effective limits of integration become much less than the original $k<k$. This means that the summation limits $L$ and $M$ of eq (23) reduce to

$$
L, M \simeq \frac{2 B\left(D+D^{\prime}\right)}{\lambda}
$$

The result (27), which holds for all separation distances for fixed $B$, is significant. It implies that the number of terms in the summation which evaluates the coupling quotient depends only on the electrical size of the antennas and not on the separation distance of the antennas. We will now show as a result of this reduction in effective limits of integration that the $\Delta k_{x}, \Delta k_{y}$ sample spacing can be increased beyond that of eq (22) to an interval independent of the separation distance d until d reaches the mutual Rayleigh distance; and thus the summation limits $L$ and $M$ can be decreased with increasing $d$ below the values given by eq (27).

Physically, eq (26) has a very simple interpretation. Referring to figure 4, it says that to a good approximation, for ordinary antennas larger than a couple of wavelengths across, only that portion of the plane-wave spectrum within the sheaf of angles mutually subtended by the smallest spheres circumscribing the radiating part of both antennas (including feeds, struts, edges and all other parts of the antenna which radiate significantly) is required to compute the coupling quotient. Thus, if the coupling quotient is desired only near $R=0$, i.e.,

$$
R<<\left(D+D^{\prime}\right),
$$

the integration limits in eq (20) need extend only over $K$ given by criterion (26). In other words, the spectrum can be set equal to zero outside the mutually subtended angle of figure 4. This means that the coupling quotient $b_{0}^{\prime}(R, d)$ computed from the limited integrations will no longer be equal, even approximately, to the actual coupling quotient for $R$ greater than about $\left(D+D^{\prime}\right)$, but will in fact become zero more rapidly beyond $\left(D+D^{\prime}\right)$. Specifically, a more detailed analysis shows that limiting the range of integration to $K\left\langle k\left(D+D^{\prime}\right) / d\right.$ also artifically band-limits the coupling quotient to

$$
R_{0}=\text { larger of }\left\{\begin{array}{c}
B\left(D+D^{\prime}\right) \\
\frac{B \lambda d}{\left(D+D^{\prime}\right)}
\end{array}\right\} \text {. }
$$

From eq (22), the sampling theorem spacing is then 

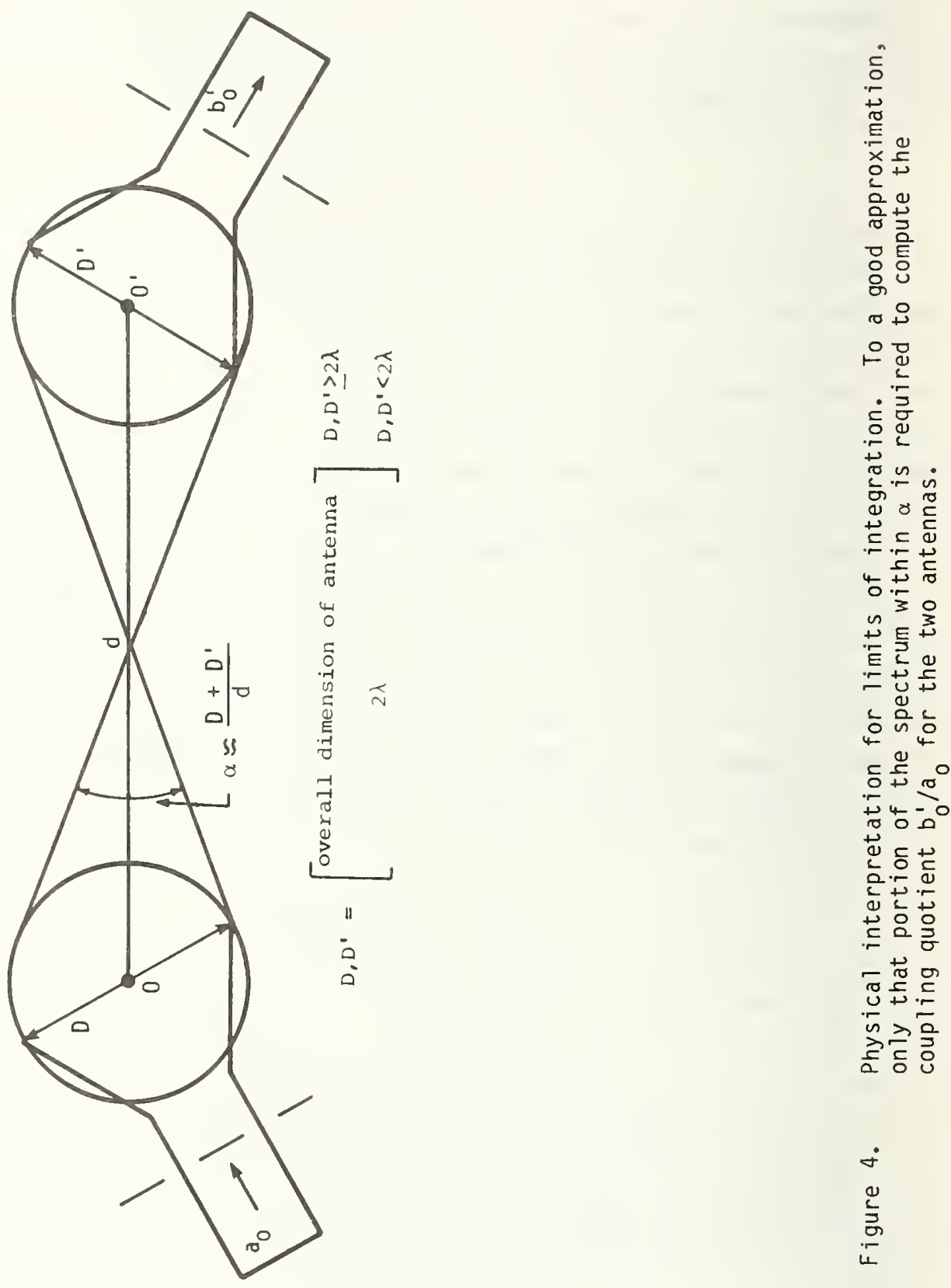

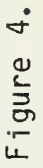




$$
\frac{\Delta k}{k}, \frac{\Delta k}{k}=\text { smaller of }\left\{\begin{array}{c}
\frac{\lambda}{2 B\left(D+D^{\prime}\right)} \\
\frac{\left(D+D^{\prime}\right)}{2 B d}
\end{array}\right\} \text {, }
$$

and from this equation and eq (26), the summation limits become

$$
L, M \simeq \text { 1arger of }\left\{\begin{array}{c}
\frac{2 B\left(D+D^{\prime}\right)^{2}}{\lambda d} \\
2 B
\end{array}\right\} \text {. }
$$

Note that when the separation d becomes larger than the "mutual Rayleigh distance," $\left(D+D^{\prime}\right)^{2} / \lambda$, only a few (2B) points of integration are required, as one might expect from physical intuition because only the near-axis pläne waves contribute to the coupling as the far field is approached.

\subsubsection{Application of the Fast Fourier Transform}

As mentioned above, eq (23) is amenable to computation by means of the efficient algorithm often referred to as the fast Fourier transform (FFT) [5]. The particular FFT algorithm we use is called FOURT and was written by Norman Brenner of MIT Lincoln Laboratories. FOURT, like all FFT algorithms, requires the summation in eq (23) to be written in a specific form, namely

$$
\frac{b_{0}^{\prime}(\underline{R}, d)}{a_{0}}=-C^{\prime} e^{-i k\left(a_{1} x+b_{1} y\right)} \frac{\left(a_{1}+a_{2}\right)\left(b_{1}+b_{2}\right)}{N_{1} N_{2}} \sum_{j_{1}=1}^{N_{1}} \sum_{j_{2}=1}^{N_{2}} A\left[j_{1}, j_{2}\right] e^{2 \pi i}\left(\frac{\left(j_{1}-1\right)\left(m_{1}-1\right)}{N_{1}}+\frac{\left(j_{2}-1\right)\left(m_{2}-1\right)}{N_{2}}\right)
$$

The definition of the various parameters in eq (32) in terms of quantities defined previously can probably be best understood by referring back to eq (20). As usual, $C^{\prime}$ is the mismatch factor (defined after eq $(8)$ ), and $(x, y)$ are the components of the transverse vector $\underline{R}$. The real numbers $\left(a_{1}, a_{2}\right)$ and $\left(b_{1}, b_{2}\right)$ define the limits of integration on $k_{x}$ and $k_{y}$; specifically,

$$
\begin{aligned}
& -a_{1} \leq \frac{k_{x}}{k} \leq a_{2} \\
& -b_{1} \leq \frac{k_{y}}{k} \leq b_{2} .
\end{aligned}
$$

$N_{1}$ and $N_{2}$ are the number of terms in the $k_{x}$ and $k_{y}$ summations respectively, and are equal to $(2 \mathrm{M}+1)$ and $(2 \mathrm{~L}+1)$ defined under eq (23). (In light of the discussion leading to eqs (26) and (31), for $\underline{R}$ near zero, $a_{1}, a_{2}, b_{1}$, and $b_{2}$ will all lie within a circle of radius $k\left(D+D^{\prime}\right) / d\left(d>D+D^{\prime}\right)$ in the $k_{x} k_{y}$ plane; and $N_{1}$ and $N_{2}$ need be no larger than about twice the $L, M$ given in eq (31).) The exponential immediately following $C^{\prime}$ in eq (32) arises from making the summation indices range only over positive integers.

In eq (32) the FFT will compute the double summation for the following values of $x$ and $y$ : 


$$
\begin{aligned}
& x=\frac{\left(-N_{1} / 2+m_{1}-1\right) \lambda}{\left(a_{1}+a_{2}\right)} \\
& y=\frac{\left(-N_{2} / 2+m_{2}-1\right) \lambda}{\left(b_{1}+b_{2}\right)},
\end{aligned}
$$

where

$$
\begin{aligned}
& m_{1}=1,2, \ldots, N_{1} \\
& m_{2}=1,2, \ldots, N_{2} .
\end{aligned}
$$

Finally, the matrix $A\left(j_{1}, j_{2}\right)$ in eq (32) needs defining:

$$
A\left(j_{1}, j_{2}\right)=\frac{k^{2}}{\gamma} \underline{f}^{\prime}\left(\phi_{p}, \theta_{p}\right) \cdot \underline{f}\left(\phi_{A}, \theta_{A}\right) e^{i \gamma d}(-1)^{j_{1}+j_{2}} \text {, }
$$

where $\left(\phi_{p}, \theta_{p}\right)$ and $\left(\phi_{A}, \theta_{A}\right)$ are determined from the transformations (13) for given Eulerian angles and $\left(k_{x}, k_{y}\right)$, which are defined in terms of $\left(j_{1}, j_{2}\right)$ by,

$$
\begin{aligned}
& \frac{k_{x}}{k}=\frac{\left(a_{1}+a_{2}\right)}{N_{1}}\left(j_{1}-1\right)-a_{1} \\
& \frac{k_{y}}{k}=\frac{\left(b_{1}+b_{2}\right)}{N_{2}}\left(j_{2}-1\right)-b_{1} .
\end{aligned}
$$

The $(-1)^{j_{1}+j_{2}}$ factor in eq (36) arises from requiring the algorithm FOURT to yield the coupling quotient directly for every value of $x$ and $y$ without the need of "rearranging." The $z$ component $\gamma$ of the propagation vector is, of course, determined from $k_{x}$ and $k_{y}$ through a simple relation, which for completeness will be repeated here:

$$
\gamma=\sqrt{k^{2}-k_{x}^{2}-k_{y}^{2}}
$$

The dot product $\underline{f}^{\prime} \cdot \underline{f}$ is also computed as explained in section 1.2.2.

In short, eq (32) for the coupling quotient between two antennas is ready for efficient evaluation on the computer using the FFT algorithm FOURT.

\subsection{Preliminary Numerical Results}

In order to build confidence in the computer program which was written to evaluate coupling products from eq (32), the far fields of two hypothetical antennas were inserted into the program. The hypothetical antennas were linearly polarized (in $x$ direction), uniform, circular aperture antennas for which the complex far-field patterns are well known in terms of simple analytic expressions involving the first-order Bessel function [6]. The radius and operating frequency of the antennas could be chosen arbitrarily along with their mutual orientation and separation. 
One check performed on the program is displayed graphically in figure 5, which shows the coupling quotient for two identical antennas facing each other in their very near field. Here the coupling should be very high, actually approaching unity when the antennas are directly aligned, as figure 5 confirms. (It should be mentioned that the curve in fig 5 and those in figs 6 and 7 took no more than a few seconds to compute.)

A second check of the computer program involves computing the coupling when the antennas are separated by a large enough distance for coupling to take place mainly between the far fields along the direction between the antennas. As mentioned in section 1.3.2., this critical distance which we call the "mutual Rayleigh distance" can be shown to be approximately $\left(D+D^{\prime}\right)^{2} / \lambda$. In figure 6 the coupling between the antennas is computed at this mutual Rayleigh distance for the antennas by two methods--first, by the FFT integration of eq (32), and then directly from the far-field coupling along the direction of separation. The close agreement between the two results again imbues confidence in the correctness of the coupling computer program.

Finally, figure 7 shows a typical coupling curve for two antennas skewed in the near field of each other. Note that a small lateral displacement appreciably less than an antenna diameter can make a $20 \mathrm{~dB}$ or more change in coupling.

In summary, the results of these and numerous other sample computations with hypothetical circular antennas yielded reasonable curves in every case; thus, we entered the experimental stage of the program, confident of the reliability of the computer program. 


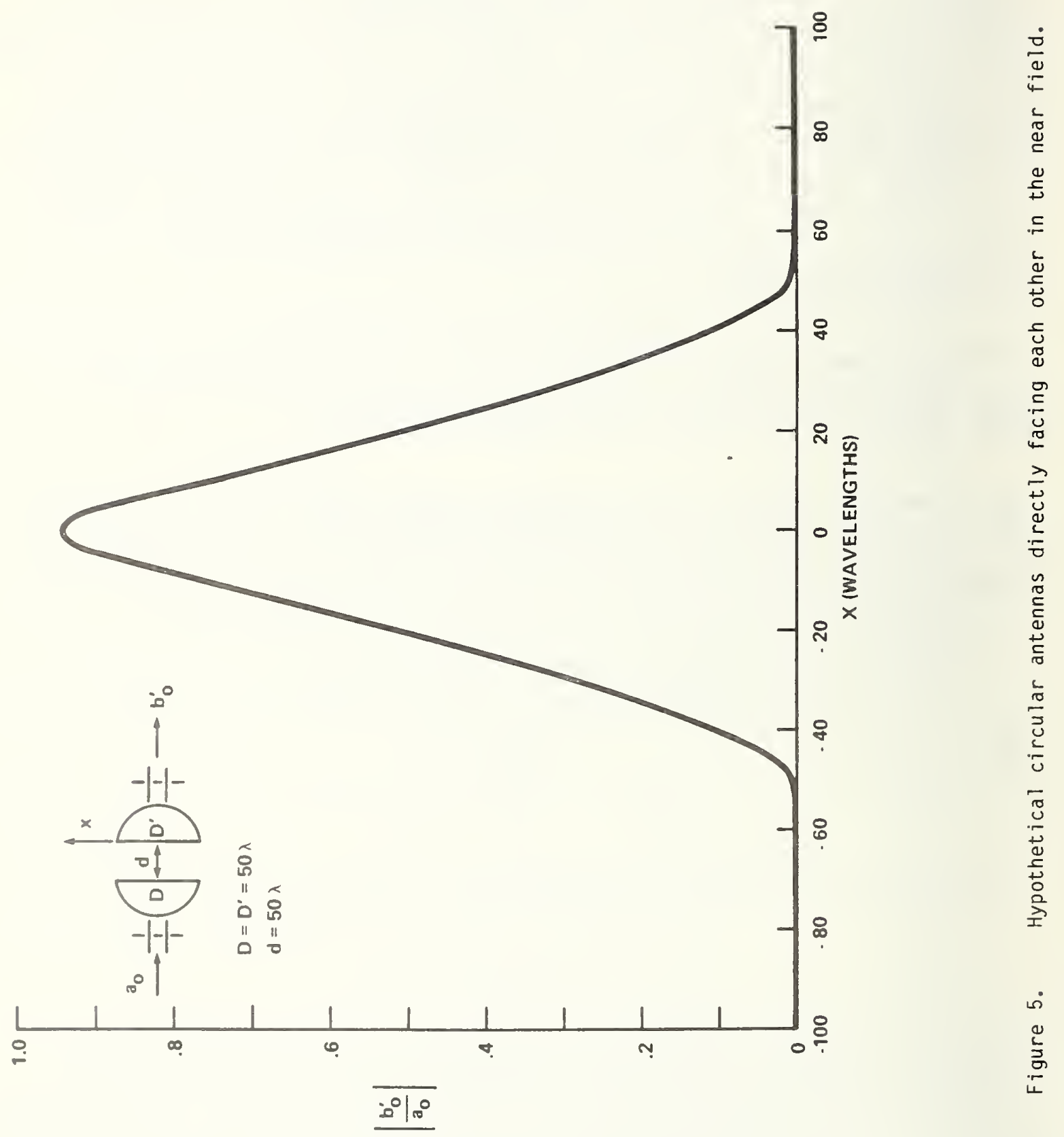



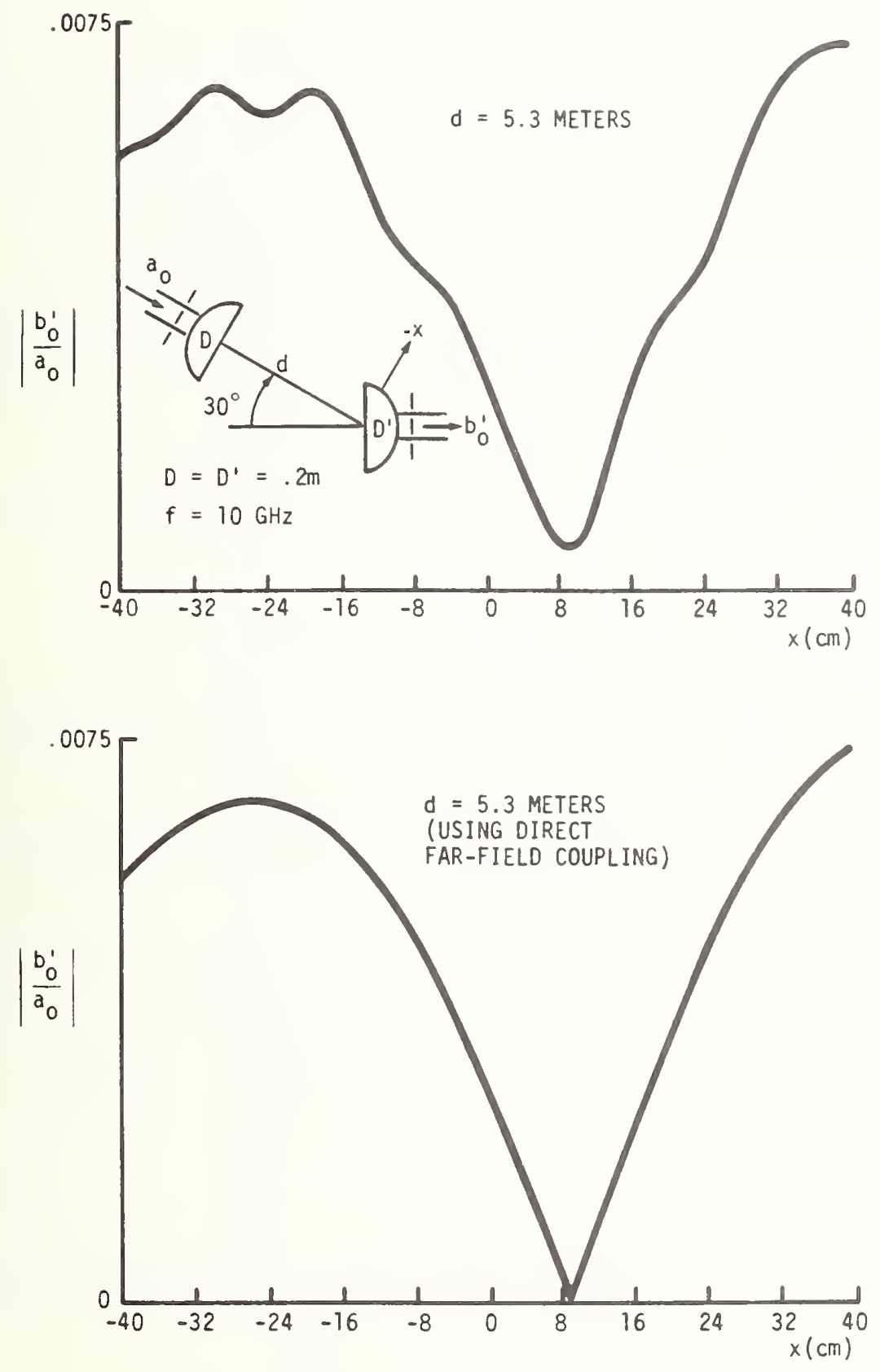

Figure 6. Coupling of circular antennas computed first using FFT integration, and then directly from far field along direction of separation. 


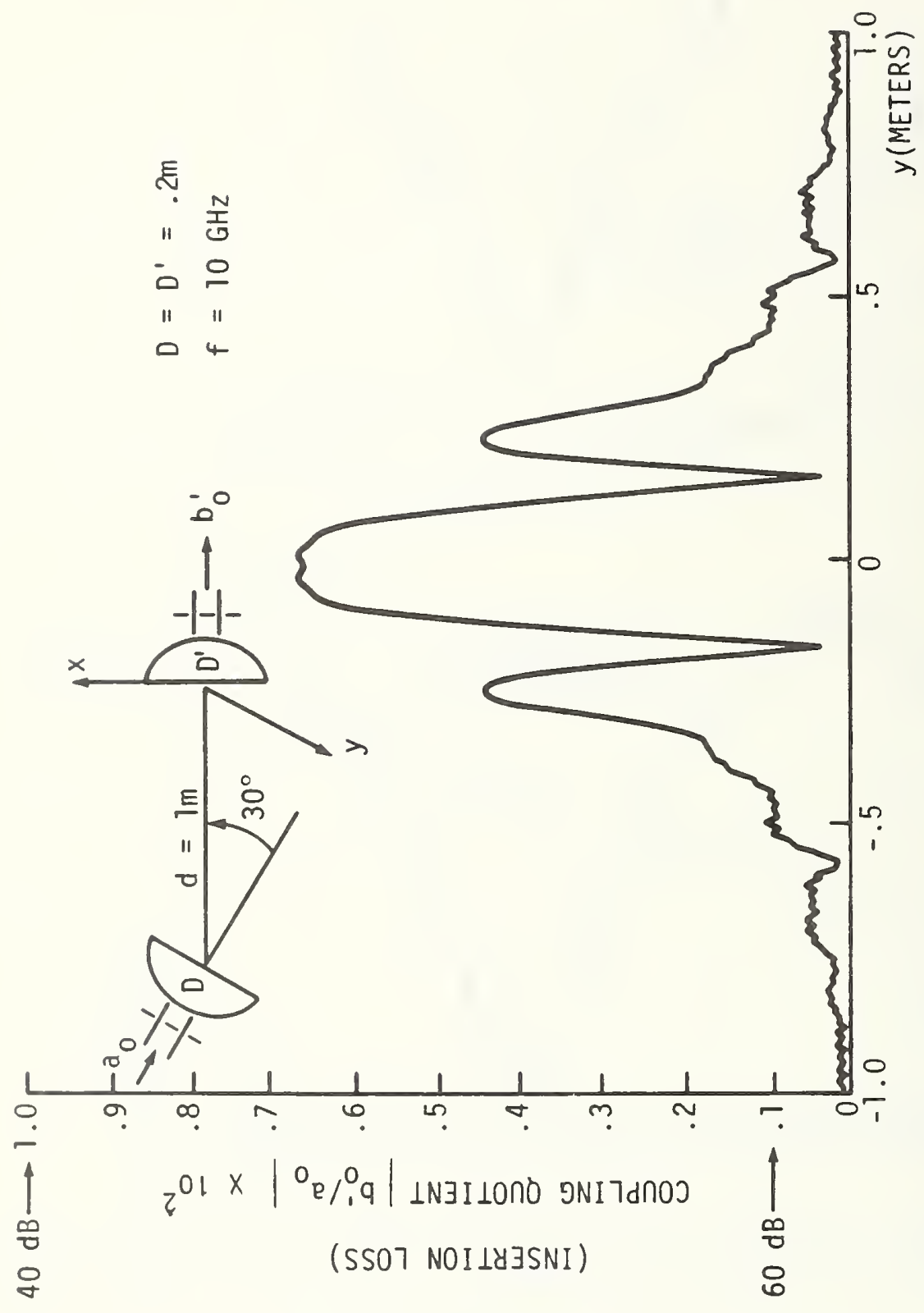

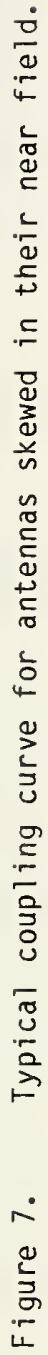




\section{TRANSFORMATION FROM FAR FIELD TO NEAR FIELD}

This section details the theory which underlies the transformation from far field to near field. As in the case of coupling between antennas, the techniques are based on the scattering matrix theory of antennas developed at NBS. A brief review of the points applicable to the calculation of near fields is presented here. For a more thorough discussion, see Kerns [1b].

We consider a finite antenna system which is located between the planes $z=z_{1}$ and $z=z_{2} ; z_{1}<z_{2}$. The fields to the right -of plane $z_{2}$ can he expressed by $a$ superposition of $\mathrm{plane}$ waves in the following form

$$
\underline{E}(\underline{r})=\frac{1}{2 \pi} \iint_{-\infty}^{\infty}\left[\underline{b}(\underline{K}) e^{i|\gamma| z}+\underline{a}(\underline{K}) e^{-i|\gamma| z}\right] e^{i \underline{K} \cdot \underline{R}} d \underline{K},
$$

where

$\underline{b}(\underline{K})$ is the spectral density function for plane waves travelling to the right (outgoing);

$\underline{a}(\underline{K})$ is the spectral density function for plane waves travelling to the left (incoming);

$\underline{k}=k_{x} \hat{e}_{x}+k_{y} \hat{e}_{y}$ is the transverse propagation vector;

$\gamma=\left(k^{2}-k_{x}^{2}-k_{y}^{2}\right)^{1 / 2}=\left(k^{2}-k^{2}\right)^{1 / 2}$ is positive real or imaginary.

$k^{2}=\omega^{2} \mu \varepsilon ;$ and

$d \underline{k}=d k_{x} d k_{y} \cdot$

Each plane wave is specified by its propagation vector

$$
\underline{\underline{k}} \pm k_{x} \hat{e}_{x}+k_{y} \hat{e}_{y} \pm r \hat{e}_{z}=\underline{k} \pm r \hat{e}_{z} \text {. }
$$

Further, each component satisfies the transversality relation

$$
\underline{k}^{+} \cdot \underline{b}=0 ; \quad \underline{k}^{-} \cdot \underline{a}=0 \text {. }
$$

We note that eq (1) indicates a Fourier transform relation exists between the electric field and the spectrum. 
A surprisingly simple relationship exists between the far-field radiation from a finite antenna and its spectrum, as noted in section 1.1.2, and is given by

$$
\underline{E}^{r}(\underline{r})=-i \gamma \underline{b}(\underline{R} k / r) e^{i k r} / r \text {. }
$$

Hence, knowledge of the far-field pattern immediately permits calculation of the spectrum, from which we can calculate the near-field pattern at any point using eq (39).

For our purposes here, we consider an antenna radiating into free space; hence, there are no waves travelling left for $z>z_{2}$. Thus, $\underline{a}(\underline{k}) \equiv 0$ and eq (39) becomes

$$
\underline{E}(r)=\frac{1}{2 \pi} \iint_{-\infty}^{\infty} c \frac{\underline{E}^{r}(\underline{r})}{\gamma} e^{i|\gamma| z} e^{i \underline{K} \cdot \underline{R}} d \underline{\underline{k}} .
$$

$C_{1}$ has been introduced as a constant which normalizes the magnitude of the far field. It will be evaluated in the following section.

\subsection{Relationship of Near-Field Intensities \\ to Power Input and Antenna Gain or Efficiency}

The constant $C_{1}$ will be determined by the power input to the antenna and the intrinsic properties of the antenna itself. We will let the property be the antenna gain as it is the one most often measured or specified. In the case of a reflector antenna, with $\underline{E}(\underline{r})$ determined by a mathematical model, we use the physical size and efficiency to provide the appropriate normalization.

Recall that, for a single antenna radiating into free space

$$
\begin{aligned}
\underline{E}(\underline{r}) & =\frac{1}{2 \pi} \iint_{-\infty}^{\infty} \underline{b}(\underline{K}) e^{i|\gamma| z} e^{i \underline{K} \cdot \underline{R}} d \underline{K} \\
& =\frac{a_{0}}{2 \pi} \iint_{-\infty}^{\infty} \underline{s}_{10}(\underline{K}) e^{i|\gamma| z} e^{i \underline{K} \cdot \underline{R}} d \underline{K} .
\end{aligned}
$$

Further, as shown by Kerns, the gain of an antenna is given by 


$$
G(\underline{k})=\frac{4 \pi y_{0} r^{2}\left|\underline{s}_{10}(\underline{k})\right|^{2}}{\eta_{0}\left(1-\Sigma_{0} p^{2}\right)}
$$

where, as in section $1, y_{0}=1 / Z_{0}$ is the admittance of free space, $\eta_{0}$ is the characteristic admittance of the feed mode, and $\Gamma_{0}$ is the antenna input reflection coefficient.

Now we are interested in normalizing our calculation to the gain in a single direction. This is usually the boresight or "on axis" direction (though in the case of a monopulse difference pattern we may need to specify the gain in a different direction.) For the antennas and models considered in this study, however, the boresight direction corresponds to the peak of the main lobe and thus makes a convenient normalization point. Solving for $\underline{s}_{10}(\underline{K}=0)$ in terms of the boresight gain and substituting into eq (42) gives

$$
\underline{E}(\underline{r})=\frac{a_{0}}{2 \pi} \sqrt{\frac{\eta_{0}\left(1-\left|\Gamma_{0}\right|^{2}\right) G(0)}{4 \pi \gamma_{0} k^{2}}} \int \underline{\hat{s}}_{10}(\underline{k}) e^{i|r| z} e^{i \underline{k} \cdot \underline{R}} d \underline{k},
$$

where

$$
\underline{\hat{s}}_{10}(\underline{K})=\frac{\underline{s}_{10}(\underline{K})}{\left|\underline{s}_{10}(0)\right|}
$$

Now, for an antenna connected to a source which delivers an average power input $P_{0}$, we have

$$
P_{0}=\frac{1}{2} \eta_{0}\left(\left|a_{0}\right|^{2}-\left|b_{0}\right|^{2}\right)
$$

but because $b_{0}=\Gamma_{0} a_{0}$

$$
P_{0}=\frac{1}{2} \eta_{0}\left|a_{0}\right|^{2}\left(1-\mid \Gamma_{0} p^{2}\right) .
$$

Substituting this into eq (44) gives

$$
\underline{E}(\underline{r})=\frac{1}{2 \pi} \sqrt{\frac{P_{0} G(0)}{2 \pi Y_{0} k^{2}}} \int \underline{\hat{s}}_{10}(\underline{K}) e^{i|\gamma| z} e^{i \underline{K} \cdot \underline{R}} d \underline{\underline{K}} .
$$


For the case of an antenna pattern determined from a model, we may estimate the gain of the antenna from its physical size and assumed efficiency. The receiving cross section $\sigma$, can be related to its physical area by the expression

$$
\sigma=\eta A,
$$

where

$$
\begin{aligned}
& \eta=\text { aperture efficiency } \\
& A=\text { physical area of the antenna. }
\end{aligned}
$$

Further, for a reciprocal antenna, gain and receiving cross section are related by

$$
G=\frac{4 \pi \sigma}{\lambda^{2}} .
$$

Finally, for a circular antenna we have

$$
G=\eta \pi^{2} d_{\lambda}^{2},
$$

where $d_{\lambda}=\frac{d}{\lambda}$ is the diameter expressed in wavelengths.

\section{PHYSICAL OPTICS MODEL FOR REFLECTOR ANTENNAS}

In order to calculate the radiated fields of a reflector antenna, it is necessary to employ some sort of approximate theory because an exact solution is essentially impossible to complete. Of several approximate theories, the one most appropriate for prediction of the antenna is main beam and near sidelobes is physical optics (PO). For farther out sidelobes, better results can usually be obtained from asymptotic theories such as the geometrical theory of diffraction (GTD).

The model employed in this work was physical optics and the basic theory will be discussed here. Several good references are available on the subject of physical optics. Here, we follow the development of Rusch $[8,9]$.

As is well known, the fields in space can be calculated if all currents are known. A general expression for these fields can be written in terms of the free-space dyadic Green's function [10]. This expression is quite complicated if we want to calculate fields at any point. However, if we desire only "far-field" expressions, considerable simplification can be made. 
We consider an arbitrary conducting surface $S$ with surface current density $\underline{J}_{S}$, as illustrated in figure 8 .

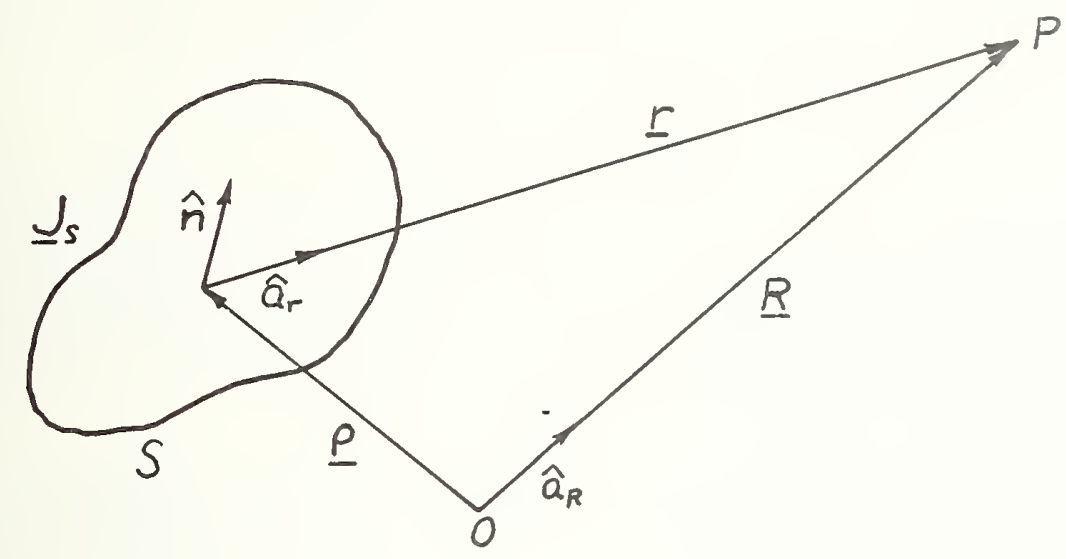

Figure 8. Geometry of vectors for surface integral.

Here, 0 is the origin of the reference coordinate system, $P$ is the field point, $\underline{R}$ is a vector which locates $P$ in the reference system, and $\hat{a}_{R}$ is a unit vector parallel to $\underline{R}$. The integration point is located by the vector $\varrho$, while the vector $\underline{r}$ designates the location of $P$ with respect to the integration point and $\hat{a}_{r}$ is a parallel unit vector.

Now, under the usual far-field assumptions $r \gg \lambda$ and $\mid \rho h_{\max } \ll R$ or $r$, we can write the electric field at $P$ as

$$
\underline{E}(\underline{R})=\frac{i \omega \mu_{0}}{4 \pi} \frac{e^{i k R}}{R} \int_{S}\left[\underline{J}_{S}-\left(\underline{J}_{S} \hat{a}_{R}\right) \hat{a}_{R}\right] e^{-i k \underline{\rho} \cdot \hat{a}_{R}} d S .
$$

This expression can be evaluated relatively easily using numerical techniques, provided that $\underline{J}_{S}$ is known. The crux of the problem, then, is the evaluation of $\underline{J}_{S}$.

A useful approximate theory for obtaining $\underline{J}_{S}$ is PO. Simply stated, P0 approximates the surface currents with those that are obtained by the assumption of a local plane-wave reflection field, i.e.,

$$
\underline{J}_{S}=2\left[\hat{n} \times \underline{H}_{i n c}\right] \text {, }
$$

where $\hat{n}$ is the unit normal to the surface and $\underline{H}_{i n c}$ is the incident magnetic field.

Numerical evaluation of the two-dimensional integral in eq (46) can be time consuming for many cases. The size of the cell required to obtain a given accuracy with the numerical integration scheme decreases as the observation point moves off axis, and may 
approach a small fraction of a wavelength. Thus, we see that calculation of the fields off axis for a large aperture antenna requires a large number of points. Further, the near-field calculations which are to be performed using the far-field patterns require a large number of individual far-field calculations.

In order to arrive at a practical model, some simplifications must be employed. The model, which is employed by the USC programs, assumes that the reflector is axially symmetric. This assumption allows the performance of the azimuthal integration in eq (46) analytically, thus reducing drastically the number of points required in the integration. Details of this simplification may be found in Rusch [8].

Another consequence of the assumption of axial symmetry is that a complete far-field pattern ( $i . e .$, specification for all values of $\emptyset$ ) requires that the field be calculated only in the E- and H-planes, i.e., $\emptyset=\pi / 2$ and 0 , respectively. The field at any point $(R, \theta, \emptyset)$ is given by

$$
\underline{E}(R, \theta, \emptyset)=\frac{e^{i k R}}{R}\left[E_{E}(\theta) \sin \emptyset \hat{a}_{\theta}+E_{H}(\theta) \cos \emptyset \hat{a}_{\emptyset}\right] .
$$

For the purposes of this study, we require the rectangular components of the antenna pattern, which are given by

$$
\begin{gathered}
\underline{E}=\frac{e^{i k R}}{R}\left[E_{E}(\theta) \cos \theta-E_{H}(\theta)\right] \cos \emptyset \sin \emptyset \hat{a}_{x} \\
+\left[E_{E}(\theta) \cos \theta \sin ^{2} \emptyset+E_{H}(\theta) \cos ^{2} \emptyset\right] \hat{a}_{y}-E_{E}(\theta) \sin \theta \sin \emptyset \hat{a}_{z} .
\end{gathered}
$$

\subsection{Physical Optics Subroutines Employed by USC}

The subroutines used to compute the PO fields of the paraboloidal reflector antennas were written by Prof. W. V. T Rusch, of the University of Southern California and obtained at a short course, Reflector Antenna Theory and Design, given in June 1976.

The subroutine package will calculate far-field patterns for an axially symmetric reflector antenna which has a circular blockage on axis caused by the feed. Further, it allows the feed pattern to be specified in the E- and H-planes independently to control the reflector illumination function.

Three options are available for the feed pattern. These are: uniform illumination, dipole illumination, and $\cos ^{n} \theta^{\prime} i l l u m i n a t i o n$ where $\theta^{\prime}$ is the angle measured from the feed axis. For this case, the feed patterns in the $\mathrm{E}$ - and $\mathrm{H}-\mathrm{pl}$ anes are given by 


$$
\begin{aligned}
& E_{E}=\cos ^{n^{E^{\prime}}} \theta^{\prime} \\
& E_{H}=\cos ^{n^{H}} \theta^{\prime} .
\end{aligned}
$$

Other parameters of the antenna which are required as input include focal length to diameter ratio, fractional diameter blockage, diameter in units of wavelength, and axial position of the feed relative to the focal point of the reflector.

The subroutines use a Romberg type of algorithm to perform the necessary integrations. This is an adaptive algorithm in the sense that it selects the necessary interval size based on a required accuracy. The result is a rapidly executing program, because advantage can be taken of the fact that rather large increments can be used near the main beam, thus reducing time to compute the far fields for these points.

If the integration routine is unable to achieve the required accuracy, either because of accumulated round-off error or because the integration range cannot be sufficiently subdivided, an appropriate error flag is set. This condition is noted in the program output, so that this data may be deleted in further calculations. Further discussion of these errors occurs in the program description.

\subsection{Test of Near-Field Program}

In order to check the operation of the near-field transformation in conjunction with the far-field PO model, a test case consisting of a 52-wavelength, uniformly 111 uminated aperture was run. Near fields were calculated in the aperture plane from the far fields calculated using P0, and were compared with the original uniform distribution. Results are shown in figure 9. As can be seen, the calculated results agree well with the uniform distribution. Note that the scale is electric field in volts/meter, not relative field in $d B$. Total variation from the original distribution is $+1.1 \mathrm{~dB},-0.55 \mathrm{~dB}$.

The ripple can be attributed to several causes. Since the PO program encounters round-off error problems for angles which lie too far off boresight, the far field must be truncated beyond a critical angle. For this example, the truncation occurred at an angle of 10.2 degrees, which was also chosen because it was a null position. Even so, eight sidelobes were included in the far-field pattern, the last one having an amplitude of about $-40 \mathrm{~dB}$ relative to the main beam. The spacing of far-field points also affects the ripple to some extent. Here, there were about 10 points per sidelobe. Finally, evanescent modes were neglected because of the point spacing chosen in $k$-space. The results do indicate that useful near fields can be calculated from the model for this case. 


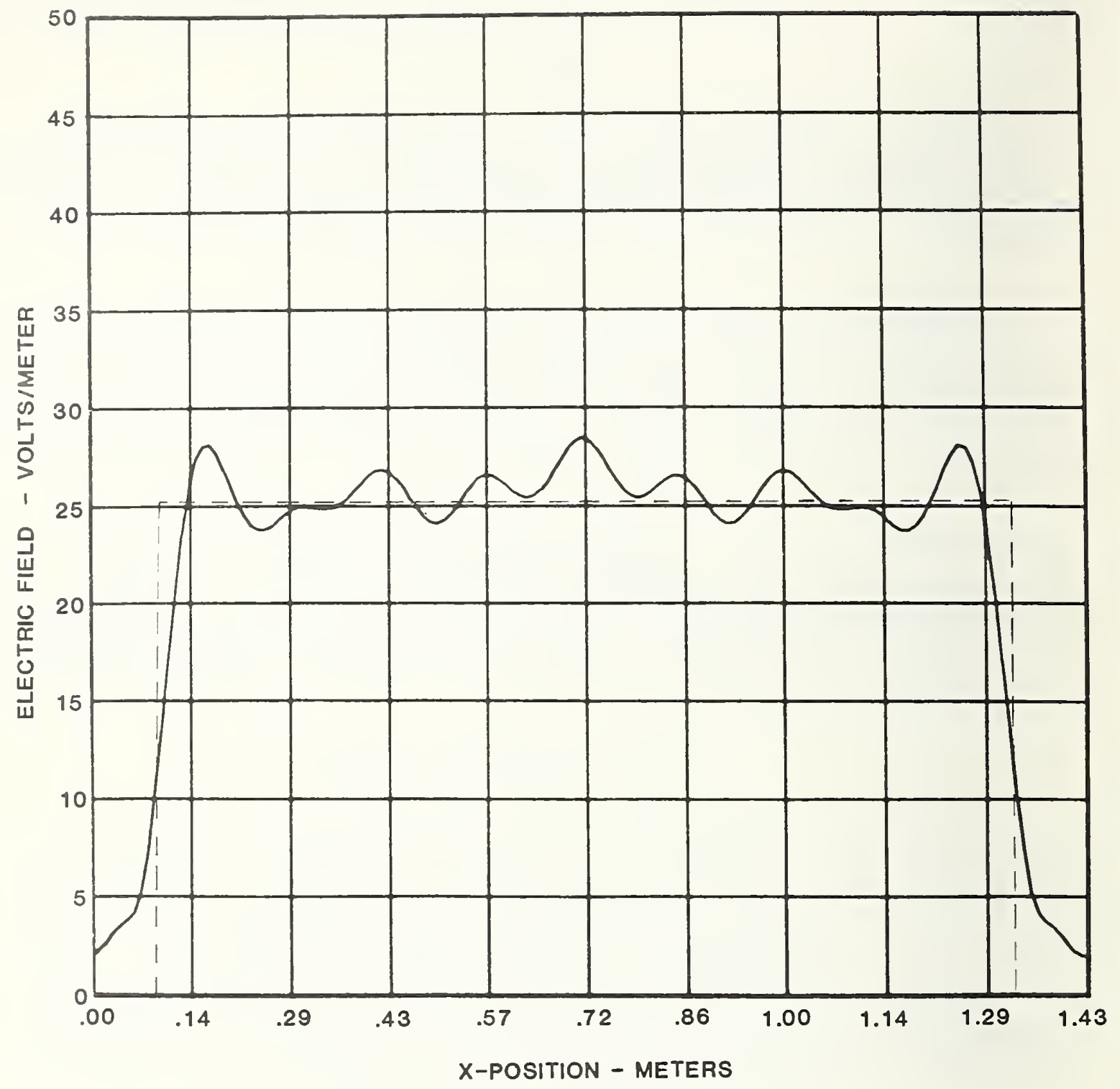

Figure 9a. Field strength in a uniformly illuminated aperture calculated using physical optics far fields. Dashed line indicates theoretical distribution. 


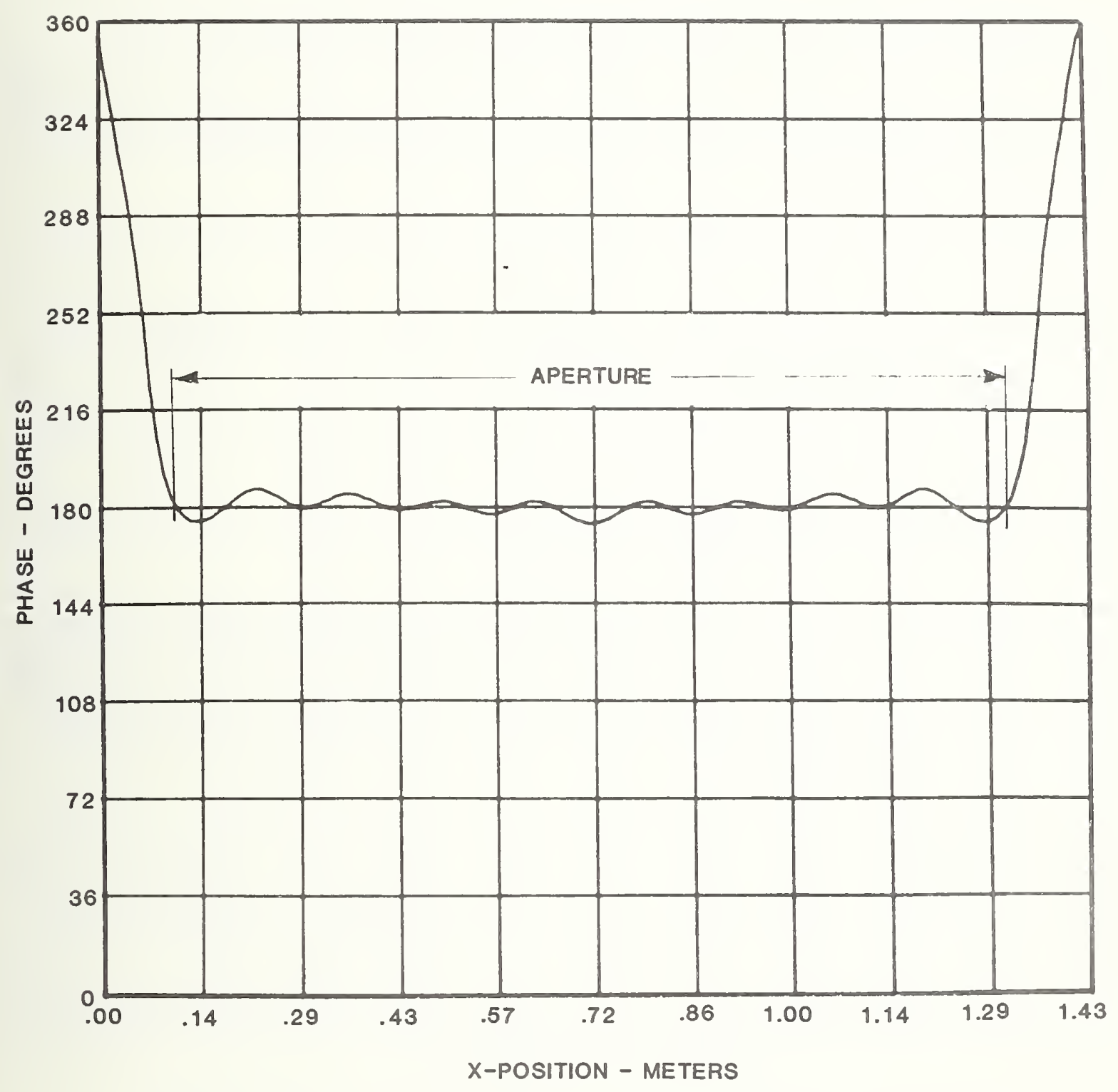

Figure 9b. Phase of field in a uniformly illuminated aperture calculated using physical optics for fields. 


\section{COMPARISON OF PHYSICAL OPTICS AND MEASURED FAR FIELDS}

As noted in section 3, the PO model represents an approximation to the true fields generated by the reflector antenna. Because of the approximations involved, it was considered desirable to compare the results obtained using a PO model to actual measured far-field patterns. Four cases were considered, and some additional experimental work was done in one case to attempt to determine the cause of observed discrepancies. The four cases are listed in table 4.1 .

TABLE 4.1

$\begin{array}{cccccccc}\text { Antenna } & \begin{array}{c}\text { Frequency } \\ \text { GHz }\end{array} & \begin{array}{c}\text { Diameter } \\ m(\lambda)\end{array} & \begin{array}{c}\text { Fractional } \\ \text { Aperture } \\ \text { Blockage }\end{array} & & n^{E} & n^{H} & \begin{array}{c}\text { Measured } \\ \text { Gain } \\ \mathrm{dB}\end{array} \\ 1 & 4.0 & 1.22(16.25) & .164 & 1.57 & 1.72 & 29.66 \\ 2 & 4.0 & 1.22(16.25) & .164 & 1.02 & 1.07 & 28.34 \\ 3 & 12.73 & 1.22(51.8) & .143 & 1.09 & 1.09 & 40.70 \\ 4 & 57.5 & .45(87.5) & .120 & & 1.10 & 46.3\end{array}$

Each antenna had an essentially circular blockage at the feed, and each had three support struts. Antennas 1,2, and 3 were essentialiy identical, being built by the same manufacturer, the only difference being in the feed. The feeds of antennas 1 and 2 were adjusted in the NBS near-field facility to obtain optimum focus and coincidence of electrical and mechanical axes.

The adjustment procedure consisted of moving the feed axially and laterally in order to obtain a minimum near-field phase curvature (focus adjustment) and a near-field phase with no linear component (boresight adjustment). It should be noted that for antennas 1 and 2, at least, it was not possible to obtain a flat phase front in both E-and H-planes. A compromise adjustment was made. Thus, either the E- or $\mathrm{H}$-plane pattern can be somewhat improved, but only at the expense of a worse pattern in the other plane. It is not known whether the problem exists in the case of antennas 3 and 4 , as these antennas had been previously measured at NBS and were not available for further experimentation.

In order to determine the parameters $n^{E}$ and $n^{H}$ for antenna 3, the dimensions of the feed were obtained and the patterns estimated using standard horn theory. For antenna 4, a cassegrain antenna, the near-field data obtained were used to estimate the parameters when the antenna was calibrated at NBS. For antennas 1 and 2, the feed patterns were measured on a far-field range before the feeds were installed on the reflector.

The far-field patterns for these antennas are shown in figures 10 to 13, with the PO predicted patterns superimposed. We note that, in general, the agreement between the 


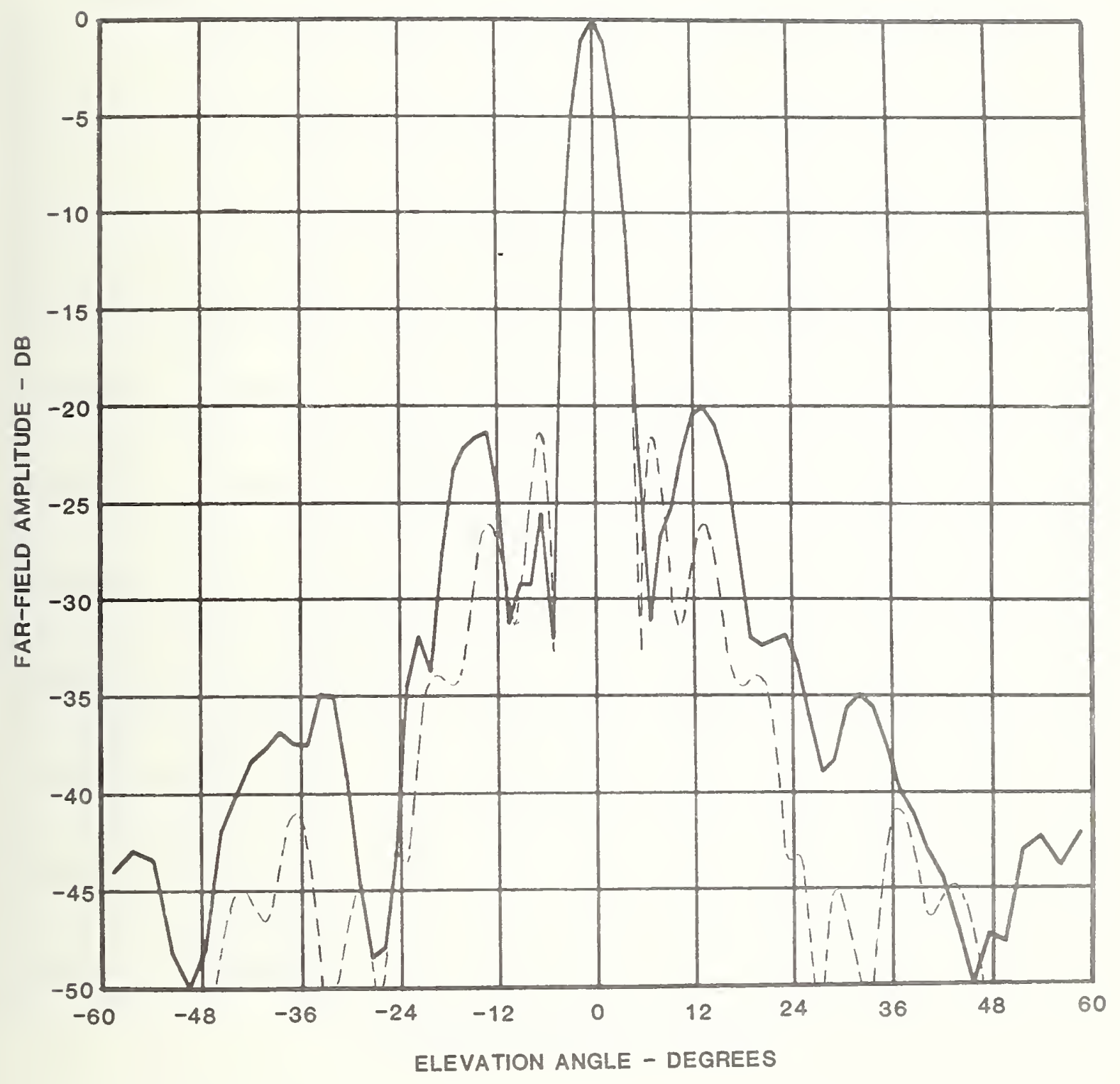

Figure 10a. Comparison of measured and calculated far-field patterns for antenna No. 1. E-plane cut, solid line - measured pattern, dashed line physical optics. 


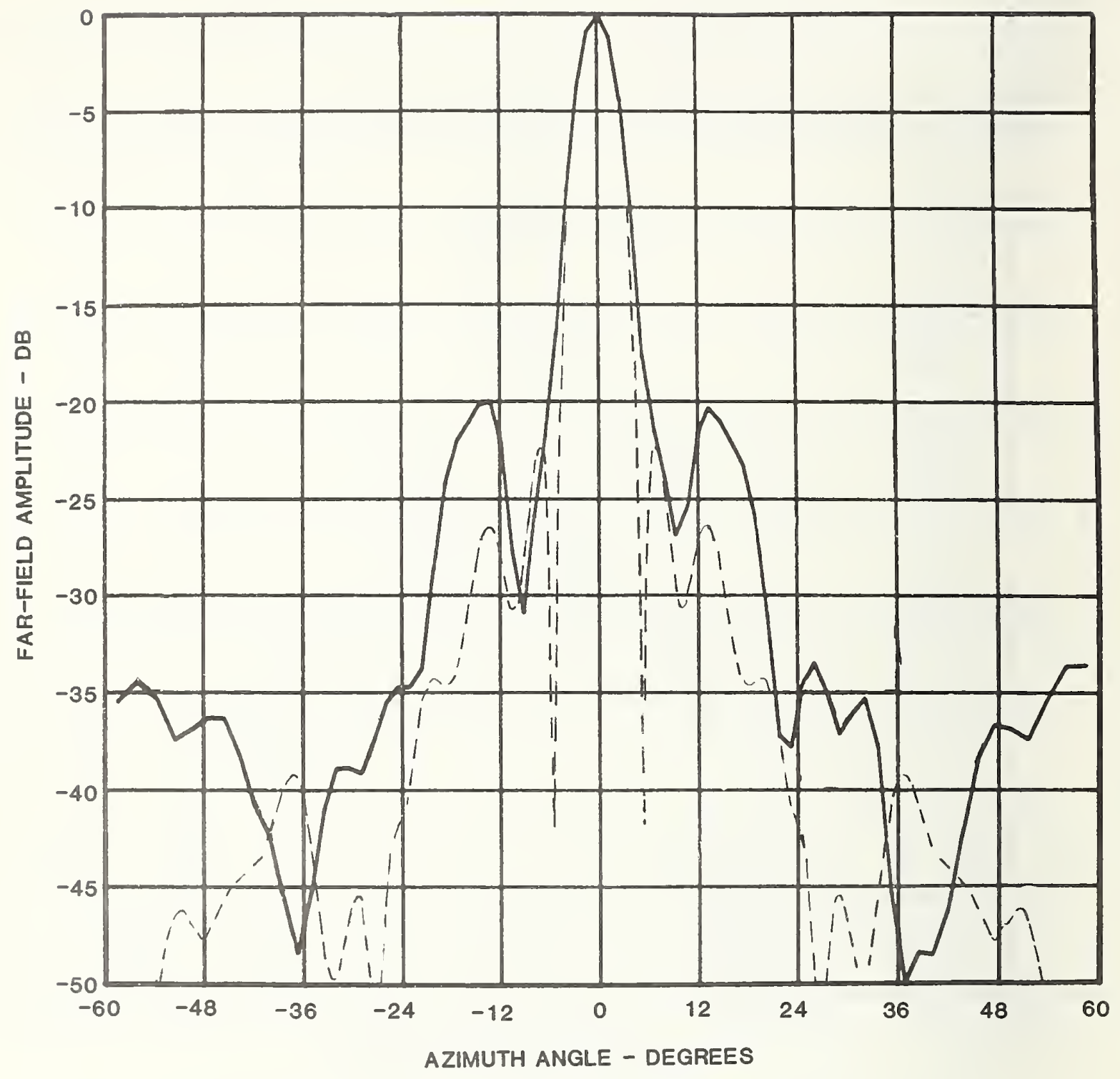

Figure 10b. Comparison of measured and calculated far-field patterns for antenna No. 1. H-plane cut, solid line - measured pattern, dashed line physical optics. 


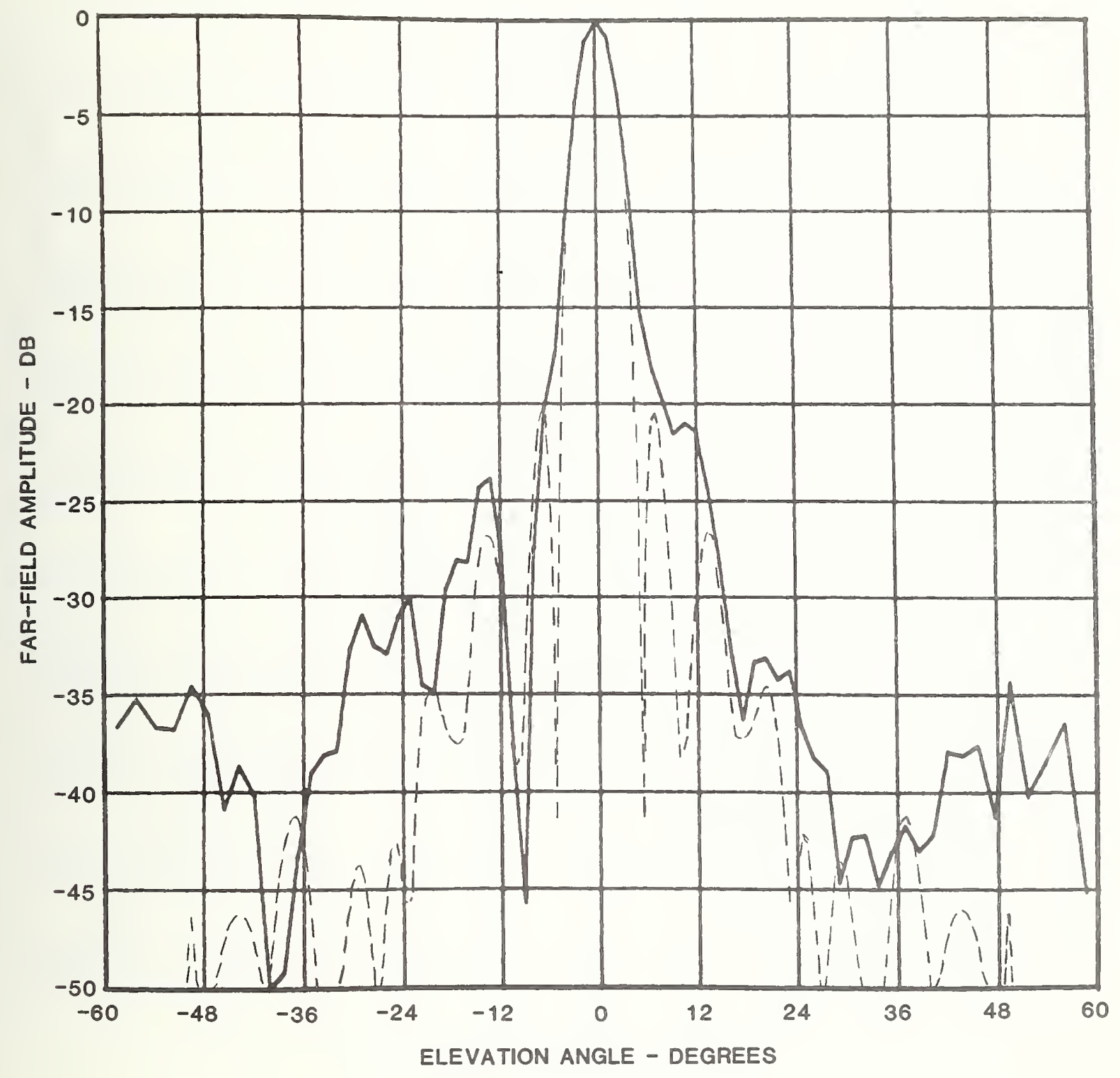

Figure 11a. Comparison of measured and calculated far-field patterns for antenna No. 2. E-plane cut, solid line - measured pattern, dashed line physical optics. 


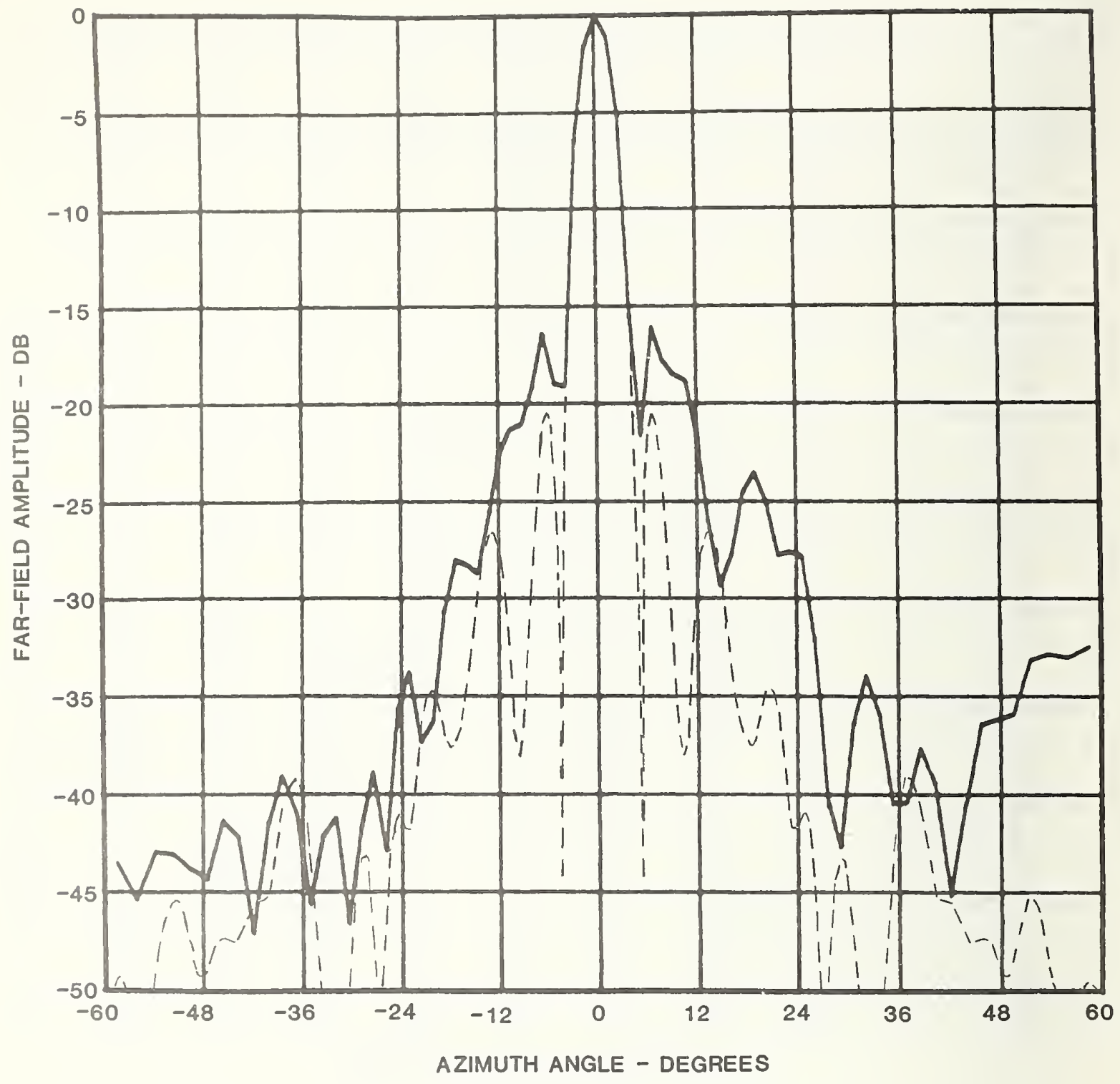

Figure 11b. Comparison of measured and calculated far-field patterns for antenna No. 2. H-plane cut, solid line - measured pattern, dashed line physical optics. 


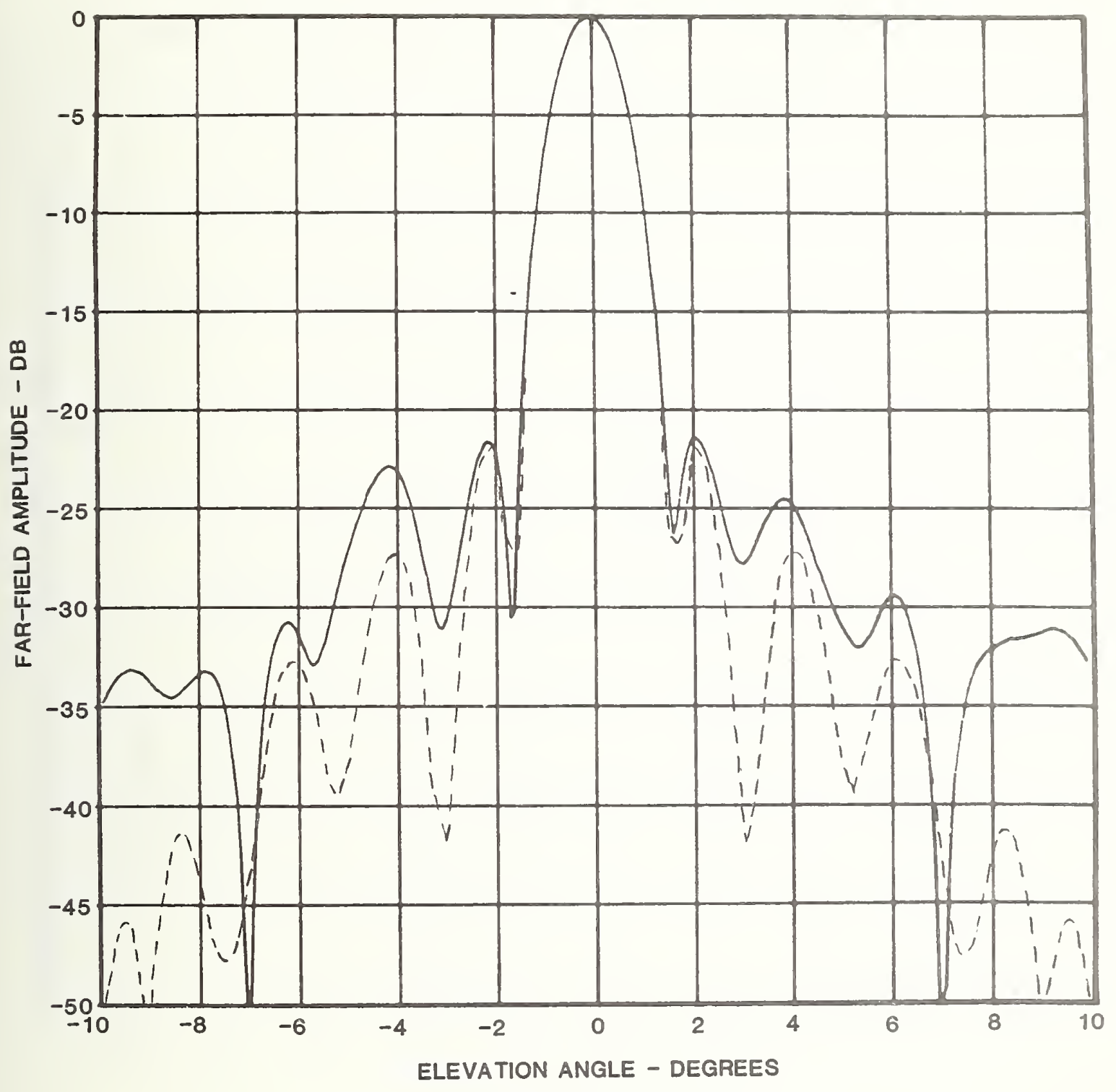

Figure 12a. Comparison of measured and calculated far-field patterns for antenna No. 3. E-plane cut, solid line - measured pattern, dashed line physical optics. 


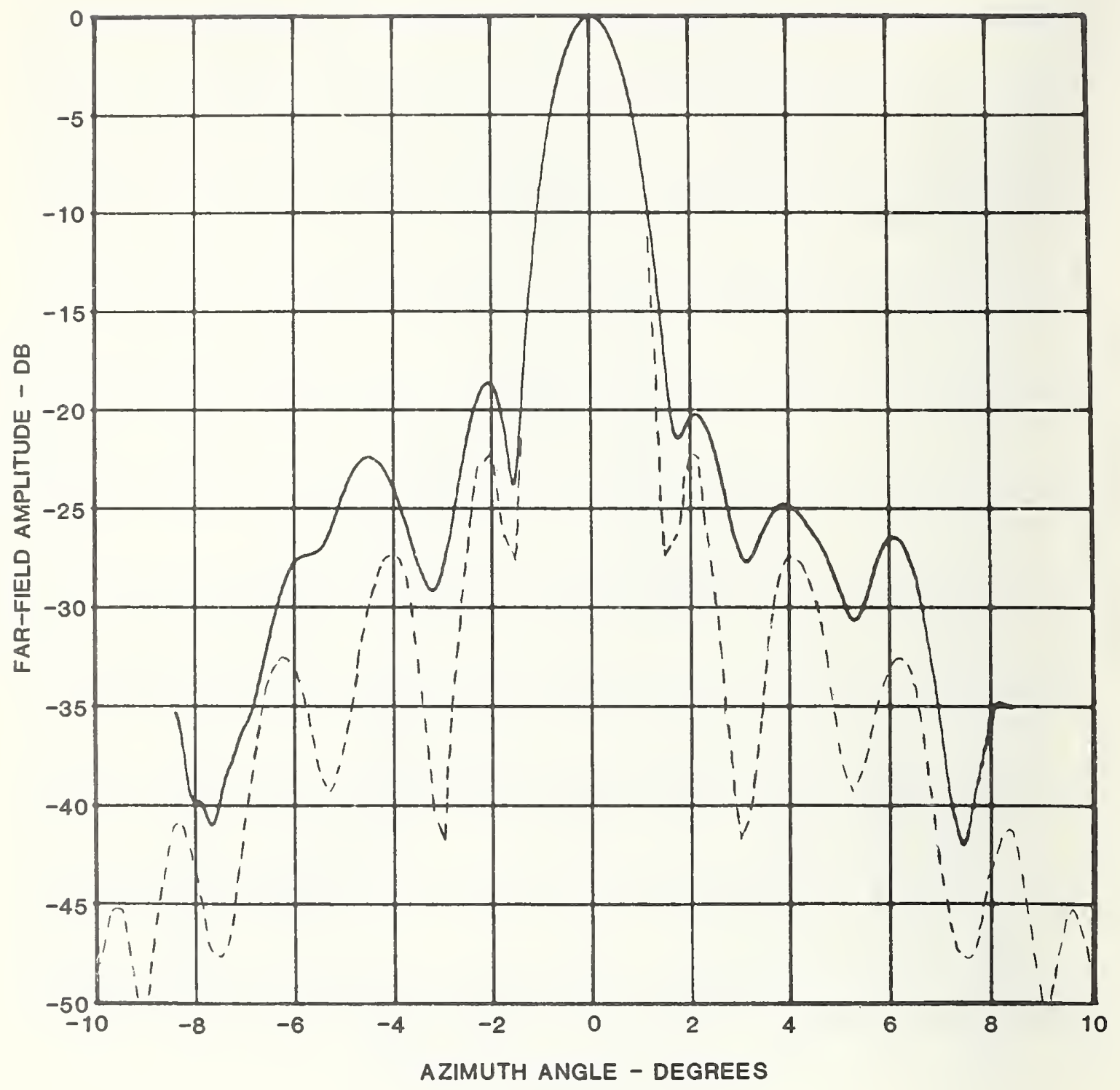

Figure 12b. Comparison of measured and calculated far-field patterns for antenna No. 3. H-plane cut, solid line - measured pattern, dashed line physical optics. 


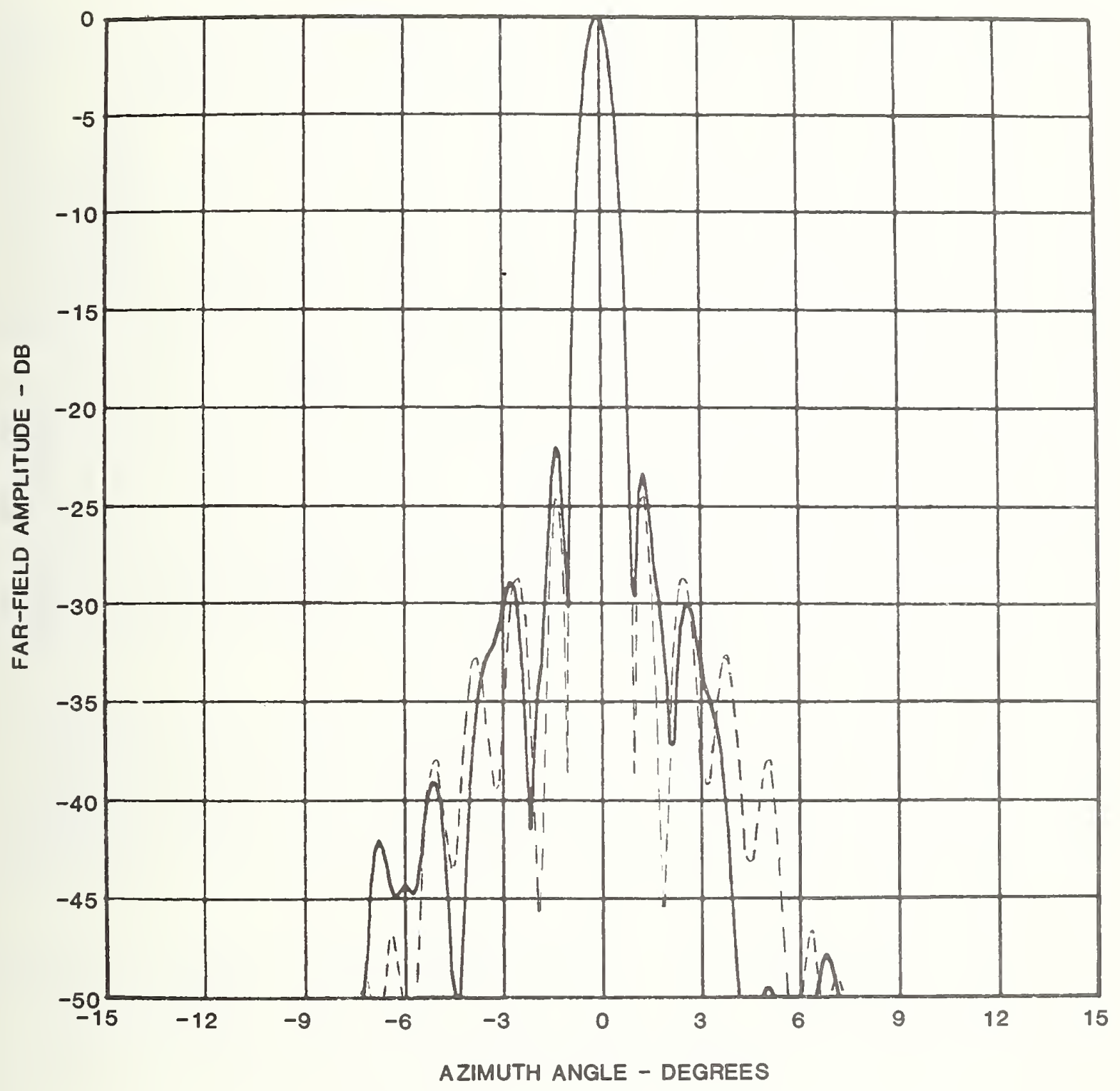

Figure 13. Comparison of measured and calculated far-field patterns for antenna No. 4. H-plane cut, solid line - measured pattern, dashed line physical optics. 
PO computations and measurements improves as the diameter to wavelength ratio increases; and further, by comparing 1 and 2, we note that a higher value of edge illumination seems to allow a better prediction.

Several possible explanations for the discrepancies exist. These can be grouped into five categories: edge effects, diffraction by struts, aperture blockage effects, back and sidelobe radiation from the feed, and violation of the assumed circular symmetry.

The first of these arises because of the sharp discontinuity in current which occurs at the edge of the reflector surface. The effect of this discontinuity is imperfectly accounted for by the PO model. In order to better describe edge effects, it is necessary to employ the geometrical theory of diffraction (GTD) or similar asymptotic theories to predict more accurately the sidelobes generated by these edge effects. To clearly see the difference between the edge as described by PO and GTD, it is useful to consider the "effective" currents which are used. These are illustrated in figure 14. We note that, in both cases, there is a sharp discontinuity in current density at the edge of the reflector surface. The GTD model includes the effect of the singularity in the current at an edge. GTD models usually assume a sharp edge. However, the antennas used in this study were made with a rolled edge as is common; and thus the normal GTD theory will not apply. The effect of the edge singularity manifests itself more as the angle off boresight increases. It is thus assumed that the use of PO rather than GTD is not significant in explaining the observed discrepancies.

The remaining processes are more likely candidates for the observed discrepancies. While blockage is taken into account, diffraction from the feed structure is not. In addition, because of the structure of the particular antennas used, multiple reflections between the feed structure and the reflector surface are likely to occur. An approximate cross section is shown in figure 15 .

In order to test the multiple reflection hypothesis, the feed support plate was lined with rf-absorbing material, and near-field scans were again taken. The resulting far fields are shown in figure 16. Note that the agreement between the P0 model and measured far fields is better. This suggests that at least part of the problem is in neglecting multiple reflections between the feed housing and the reflector.

The struts were now covered as shown in figure 17 to try to minimize diffraction by them. Results of this test showed an increase in the discrepancy between experiment and theory as shown in figure 18. However, this should not be taken to mean that strut reflection is negligible because, as can be noted in the photograph, there is significantly more blockage for rays travelling off axis than in the uncovered strut case. A better method for determining the strut diffraction effect experimentally would be to support the feed with dielectric material and measure patterns in this configuration. The asymetry observed in the E-plane pattern is an indication of significant strut effects. 

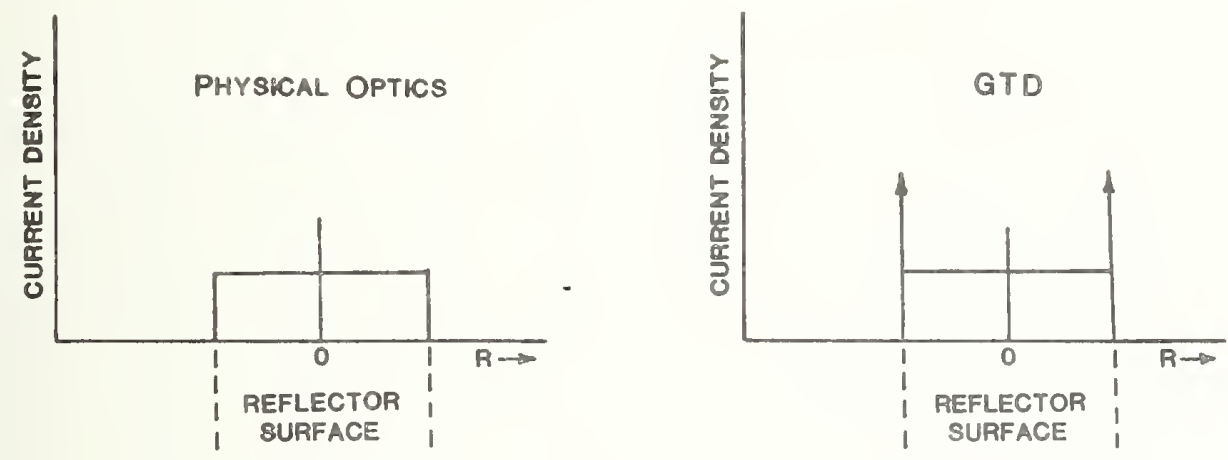

Figure 14. Comparison of effective current distribution used in physical optics and geometrical theory of diffraction calculations. (Uniform distribution assumed).

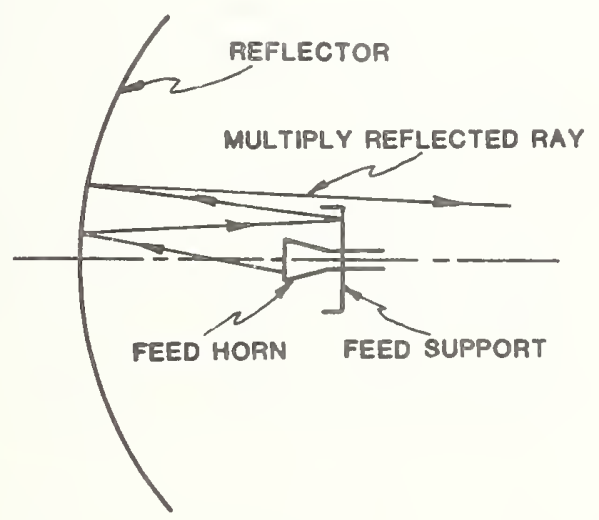

Figure 15. Diagram of multiple reflections involving feed structure. 


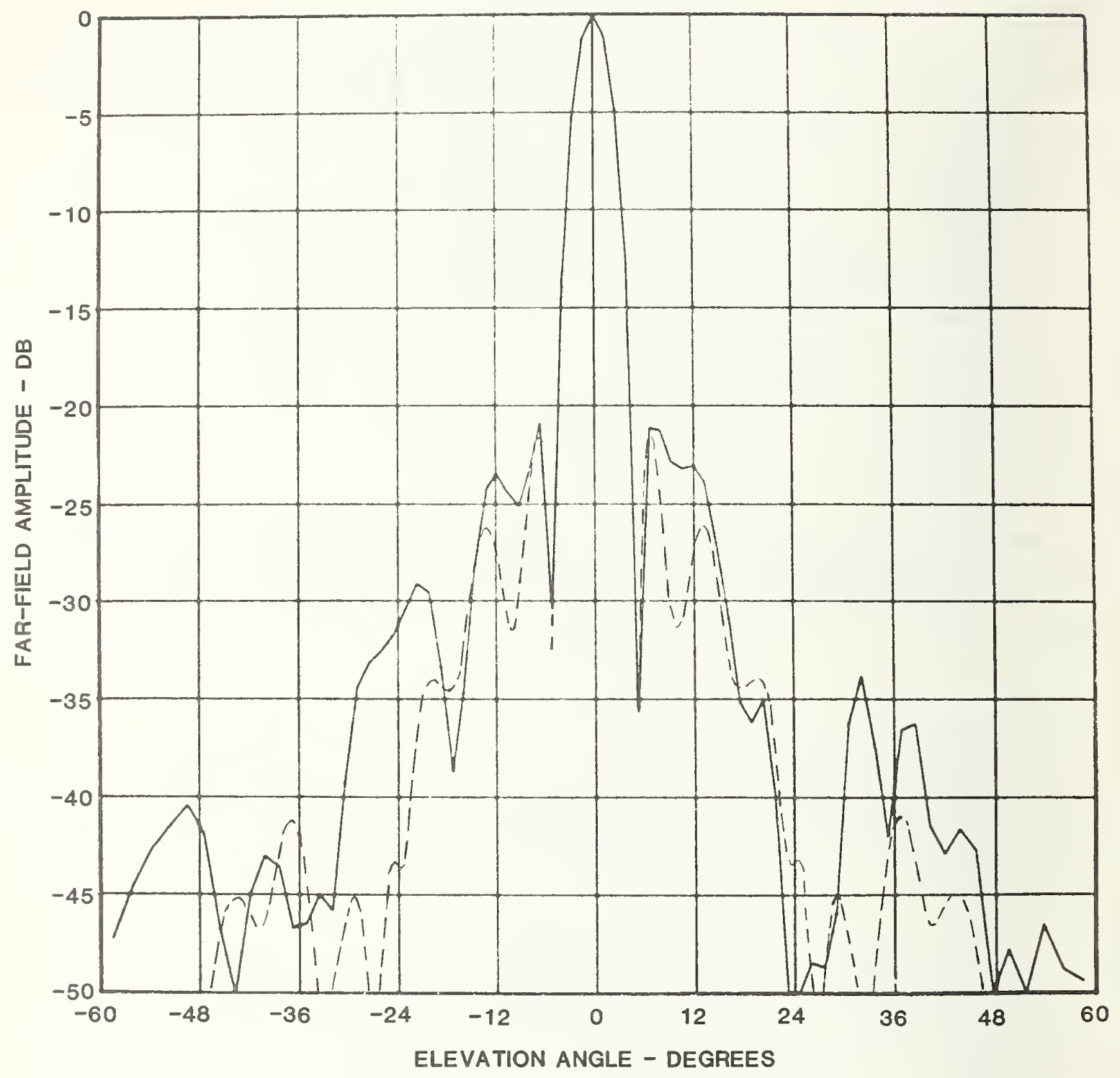

Figure 16a. Comparison of measured and calculated far-field patterns for antenna No. 1 with feed region covered with absorber. E-plane cut, solid curve measured pattern, dashed curve - physical optics. 


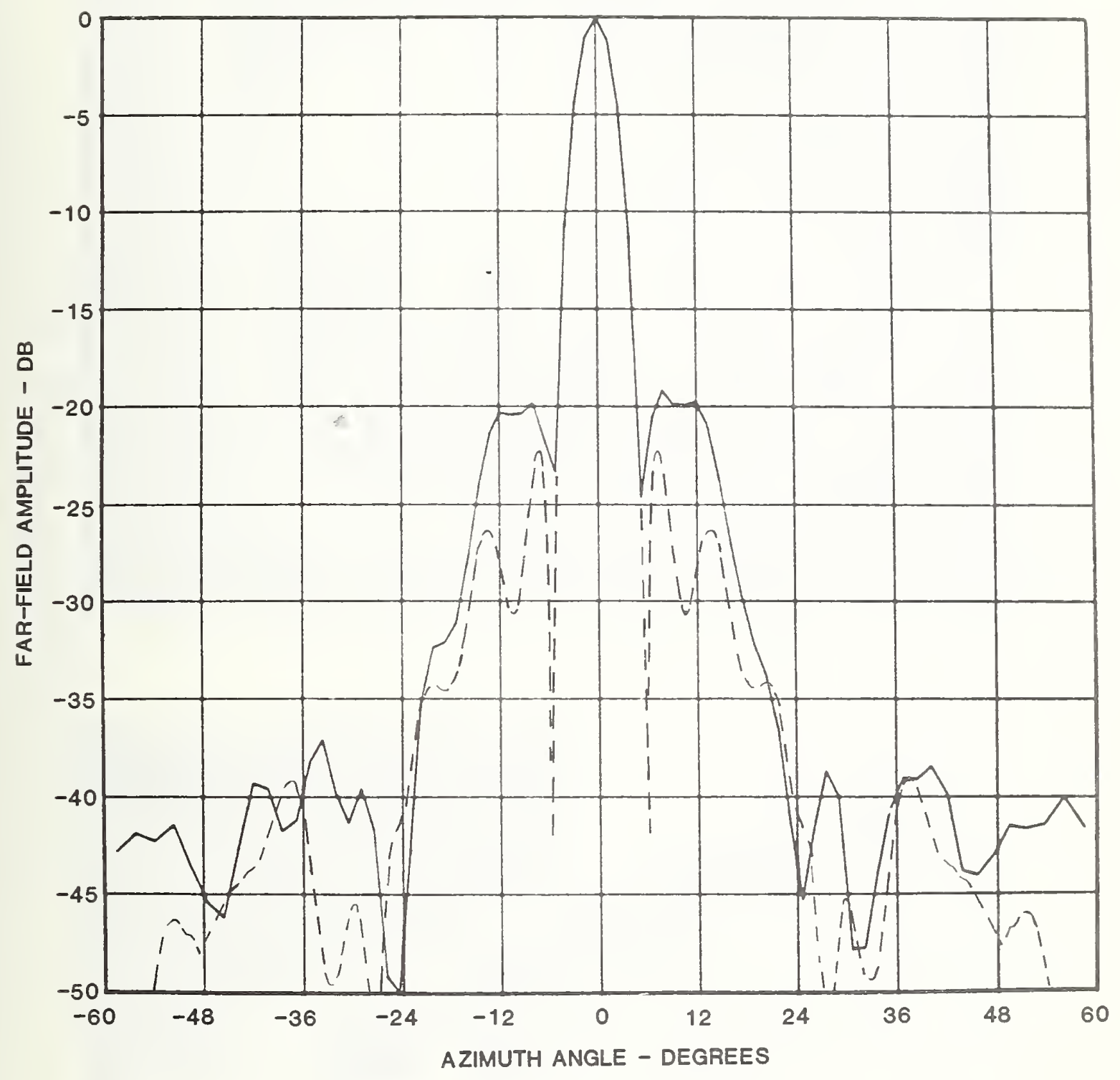

Figure 16b. Comparison of measured and calculated far-field patterns for antenna No. 1 with feed region covered with absorber. H-plane cut, solid curve measured pattern, dashed curve - physical optics. 


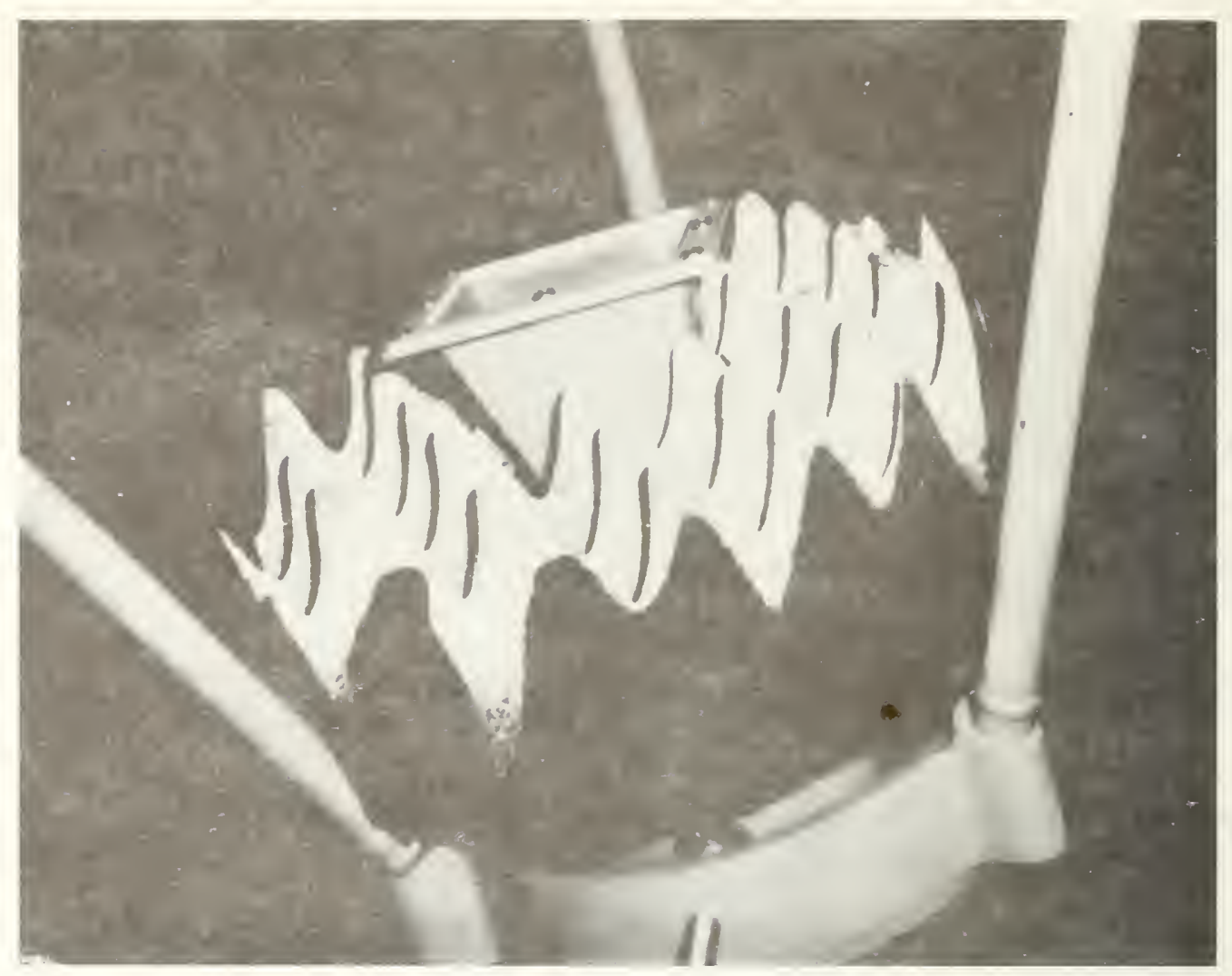

Figure 17a. Feed region of antenna with absorber collar.

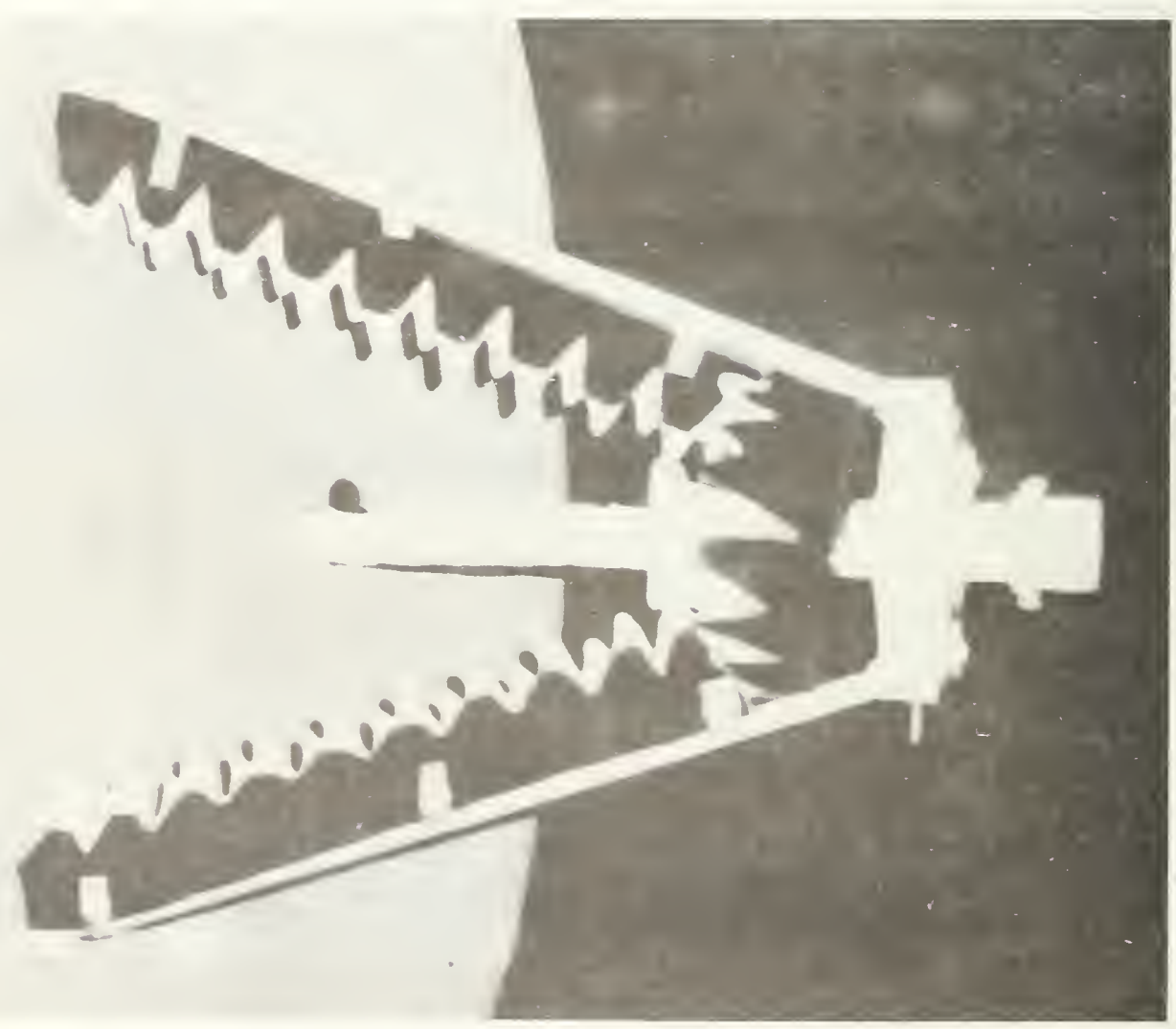

Figure 17b. Feed support struts with absorber attached. 


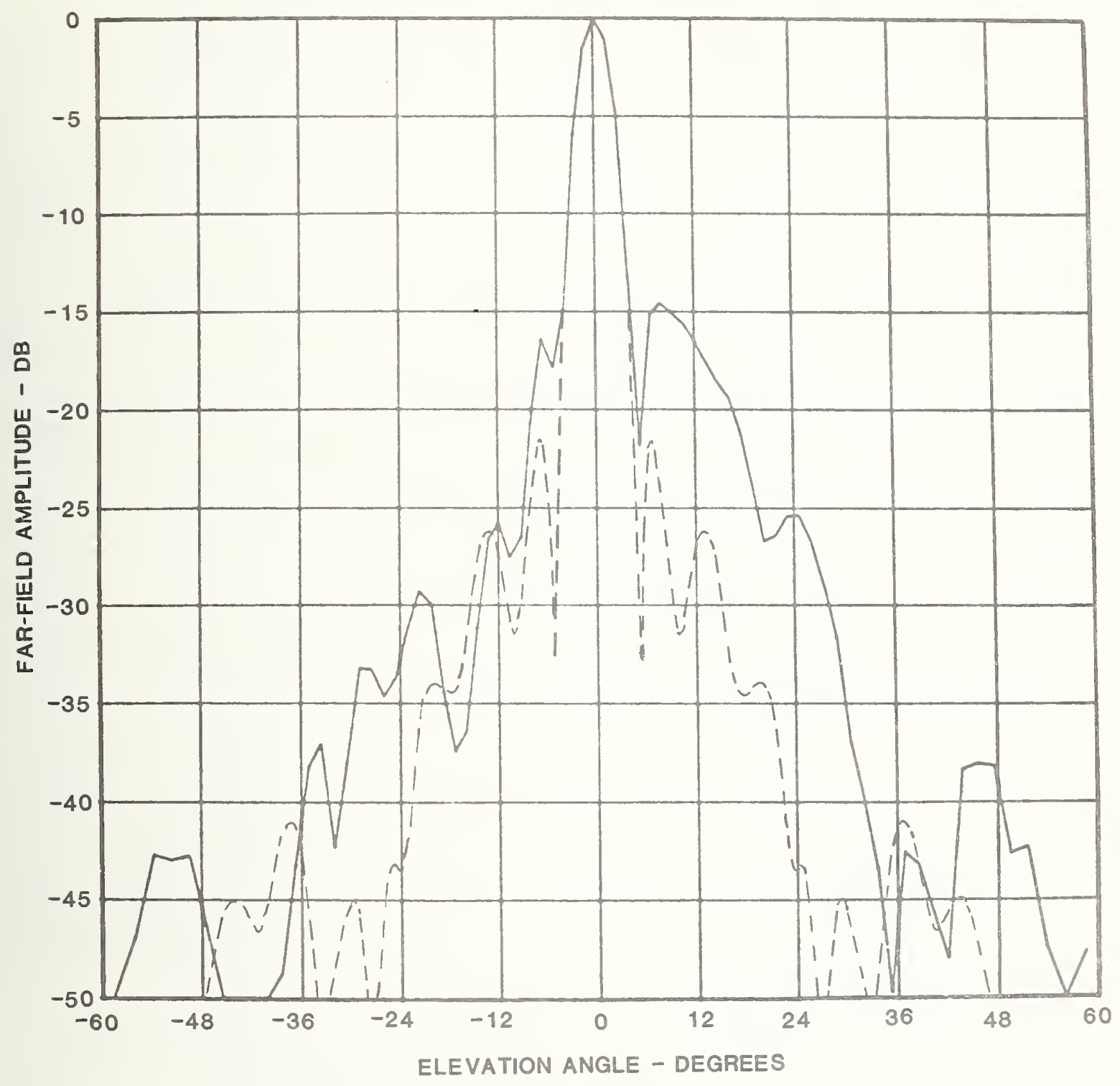

Figure 18a. Comparison of measured and calculated far-field patterns for antenna No. 1 with feed region covered with absorber. E-plane cut, solid curve measured pattern, dashed curve - physical optics. 


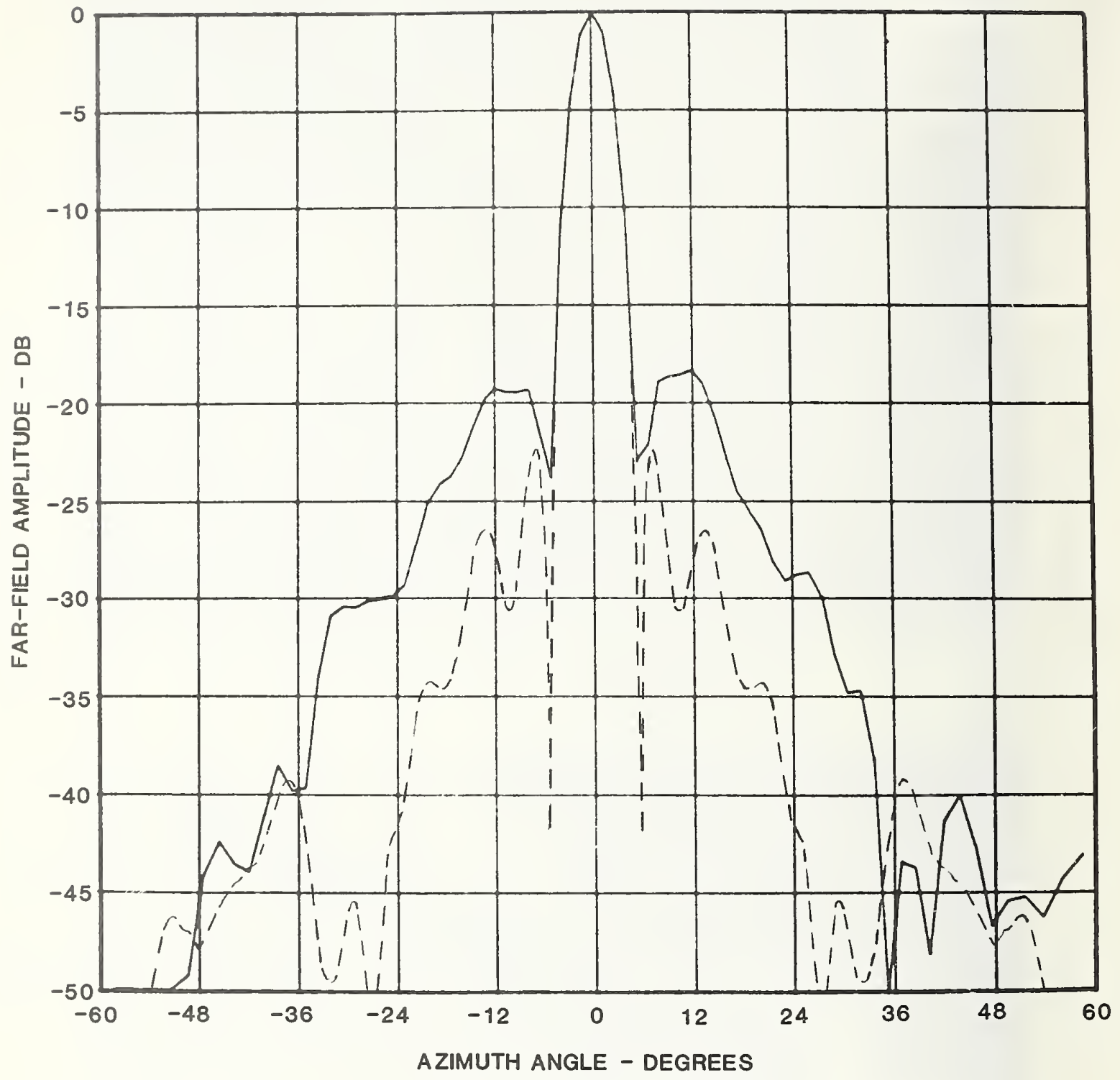

Figure 18a. Comparison of measured and calculated far-field patterns for antenna No. 1 with feed region covered with absorber. H-plane cut, solid curve measured pattern, dashed curve - physical optics. 
Backlobe radiation from the feed antenna is not considered. It is difficult to estimate the magnitude of this effect. While patterns were taken for the feeds of antennas number 1 and 2, it is in a completely different mounting structure when in place in the antenna; and, as a result, the pattern in the rear hemisphere for the feed will not give any valid data about its back lobes.

The following general conclusions can be stated concerning the usefulness of this particular PO model.

1. The model appears to give better results for larger $D / \lambda$ ratios.

2. Sidelobe positions are fairly accurately predicted for the first few sidelobes.

3. The magnitudes of the predicted sidelobes can be as much as several dB off for small $(\langle 50 \lambda)$ antennas.

4. A contributor to the observed differences in the case of antenna 1 (and also 2 because its construction was the same) is multiple reflections between the reflector surface and feed structure.

5. For far sidelobe regions (beyond 4 or 5 lobes) it appears that a better model such as a PO-GTD combination should be employed.

6. A model which takes struts into account would be useful.

Because the theoretical model is used to predict near-zone fields and coupling, it is useful to consider the effect of discrepancies between the modeled and actual fields on the prediction of near-fields and coupling.

For determination of the near-field radiation in front of the antenna, it is expected that the sidelobe discrepancies will have a negligible effect. The major source of error will occur because the true gain is not known and must be estimated. The current P0 model will not give results in the region far off boresight or in the back direction.

The coupling results will be affected by the sidelobe region, however. Calculation of the coupling depends on that portion of the far field of each antenna which is subtended by the other; hence, the sidelobe structure is important. Because the locations of the sidelobes are accurately predicted, the basic structure of the coupling as a function of 
relative position of the two antennas will be retained. Any errors in the magnitude of the far field predicted by PO will be carried over into the coupling ratio.

\section{COMPARISON OF PREDICTED AND MEASURED NEAR-FIELD COUPLING}

In order to utilize the near-field coupling program (CUPLNF) to predict actual near-field coupling, the two C-Band reflector antennas (numbers 1 and 2 of table 4.1) which were modeled using $\mathrm{PO}$, were set up to measure the near-field coupling directly for various relative orientations and separations. The frequency of operation was $4.0 \mathrm{GHz}$ which gives

a diameter of 16.25 wavelengths and a combined or mutual Rayleigh distance $\left(D_{1}+D_{2}\right)^{2} / \lambda$ of about 80 meters.

The antennas were mounted on movable wooden towers at a height of about 7 meters above the ground. The coupling was measured as a function of separation distance for separations ranging from 1 to 8.5 meters and for three relative orientations of the antennas. This procedure also gives a measure of the level of multiple reflections between the antennas (which are neglected in the calculations).

A photograph of the experimental setup is shown in figure 19, and figure 20 illustrates schematically the three relative orientations employed.

For cases two and three, the angle of the receiving antenna was varied over approximately a $\pm 4^{0}$ range at a fixed separation of 3.5 meters to test the coupling as a function of angle.

Small angles were deliberately chosen for two reasons. Because the PO model used here does not perform well in the sidelobe region, the measurements must be restricted to small angles so the model can successfully predict coupling from boresight. Further, the planar scan data yields far fields which are valid only to about $45^{\circ}$ to $50^{\circ}$, and, this too, limits the angles. For wider angle coverage, nonplanar scanning techniqes such as cylindrical or spherical would prove useful.

It should be noted that in case 1 , the primary source of coupling is the interaction of the main lobes of the two antennas. Case 2 corresponds to the main lobe of the transmitting antenna interacting with the sidelobes of the receiving antenna. In case 3 , the sidelobes of each antenna interact with each other.

Calculation of the coupling between the antennas was carried out for five separations in the range 1.5 to 7.5 meters for each case measured. Far fields used as input were from two sources. The experimentally determined far fields obtained from transformation of near-field data were used in one set of calculations, and the far fields obtained from the model using the adapted USC PO subroutines were used in the other calculations. 


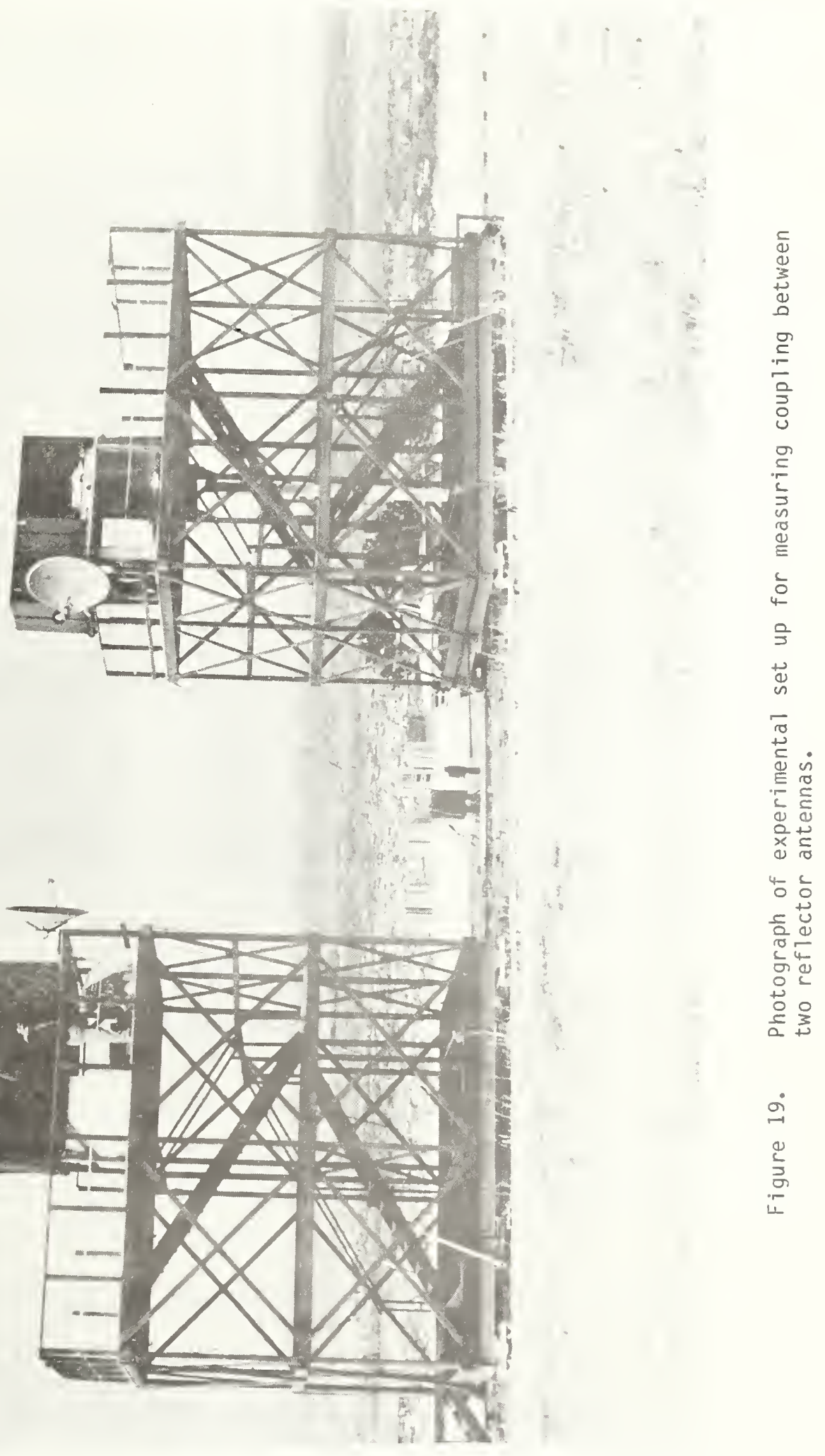




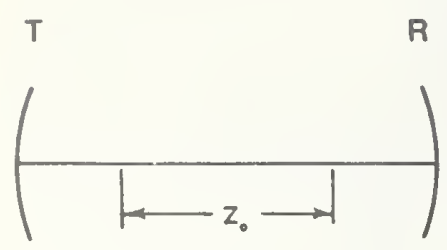

CASE 1

$$
\begin{array}{ll}
\phi_{t}=0^{\circ}, & \phi_{r}=0^{\circ} \\
\theta_{t}=0^{\circ}, & \theta_{r}=0^{\circ} \\
\psi_{t}=0^{\circ}, & \psi_{r}=0^{\circ}
\end{array}
$$

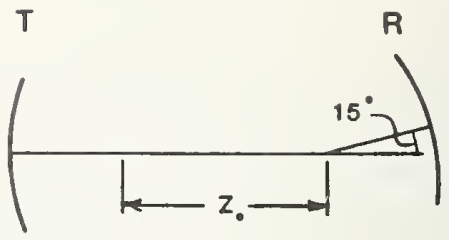

CASE 2

$$
\begin{array}{ll}
\phi_{t}=0^{\circ}, & \phi_{r}=180^{\circ} \\
\theta_{t}=0^{\circ}, & \theta_{r}=15^{\circ} \\
\Psi_{t}=0^{\circ}, & \Psi_{r}=180^{\circ}
\end{array}
$$

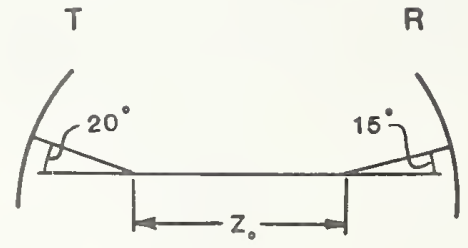

CASE 3

$$
\begin{array}{ll}
\phi_{t}=0^{\circ}, & \phi_{r}=180^{\circ} \\
\theta_{t}=20^{\circ}, & \theta_{r}=15^{\circ} \\
\psi_{t}=0^{\circ}, & \psi_{r}=180^{\circ}
\end{array}
$$

Figure 20. Schematic showing relative orientations of antennas for the three test cases. 
The results of the three cases are shown in figures 21 to 23 . In each case, the envelope of the measured data is shown, rather than the actual data, which consists of approximately sinusoidal oscillations of period $\lambda / 2$ superimposed on the data which arise because of multiple reflections between the two antennas.

We note fairly good agreement between the measured data and that predicted using measured far fields except in the case of the $\left(00,15^{0}\right)$ data. This disagreement wi 11 be discussed shortly. In the $\left(0^{0}, 0^{0}\right)$ case, the prediction using actual far-field data is approximately $0.5 \mathrm{~dB}$ low, and follows the shape of the average of the measured data very wel1. In the $\left(20^{\circ}, 1^{\circ}\right)$ case, we again ob-serve fairly good agreement between the shape of the predicted and measured curves with an average error of about $2 \mathrm{~dB}$. As in the case of the measurement of low sidelobes, this error is not unacceptable. It might be expected that a greater error would occur when the sidelobes are interacting because of their complicated structure and resultant sensitivity to orientation. While every effort was made in the experimental procedure to ensure accurate positioning, the accuracy was probably no better than $1 / 2^{\circ}$ about a 71 three axes.

We now discuss the $\left(0^{0}, 15^{\circ}\right)$ case where agreement is not good. Here, we suggest that slight misalignment may be the primary cause. In the rotation performed at a separation of 3.5 meters, a peak of $-25.2 \mathrm{~dB}$ occurred at about $12.0^{\circ}$. Calculations show that a peak in the predicted coupling occurs at an angle of 12.40 with a magnitude of $-26.4 \mathrm{~dB}$. Predicted and observed nulls also occur at about $20^{\circ}$ to $22^{\circ}$, though the magnitude comparison of the null depth is not so good. Because of multiple reflections and multipath and because the cross-polarized component is not included in the calculations, null comparisons cannot be expected to be so good as that observed at relative maxima. It would thus appear that the discrepancy at $\left(0^{0}, 15^{\circ}\right)$ can be explained by a small error in orientation.

\section{CONCLUSIONS AND RECOMMENDATIONS}

Programs and subroutines were written to calculate near fields of reflector antennas and to calculate mutual coupling between antennas whose separation and orientation are arbitrary. The basic data required for these calculations are the twó-dimensional complex far-field patterns of the antennas involved.

Documentation for the programs including listings and sample input and output are given in Appendices $A$ and $B$.

It was seen that the coupling program provides good results if proper far fields are used as input data. When a model such as the physical optics discussed here is employed, the coupling program fails to adequately predict the coupling for off-axis directions. 


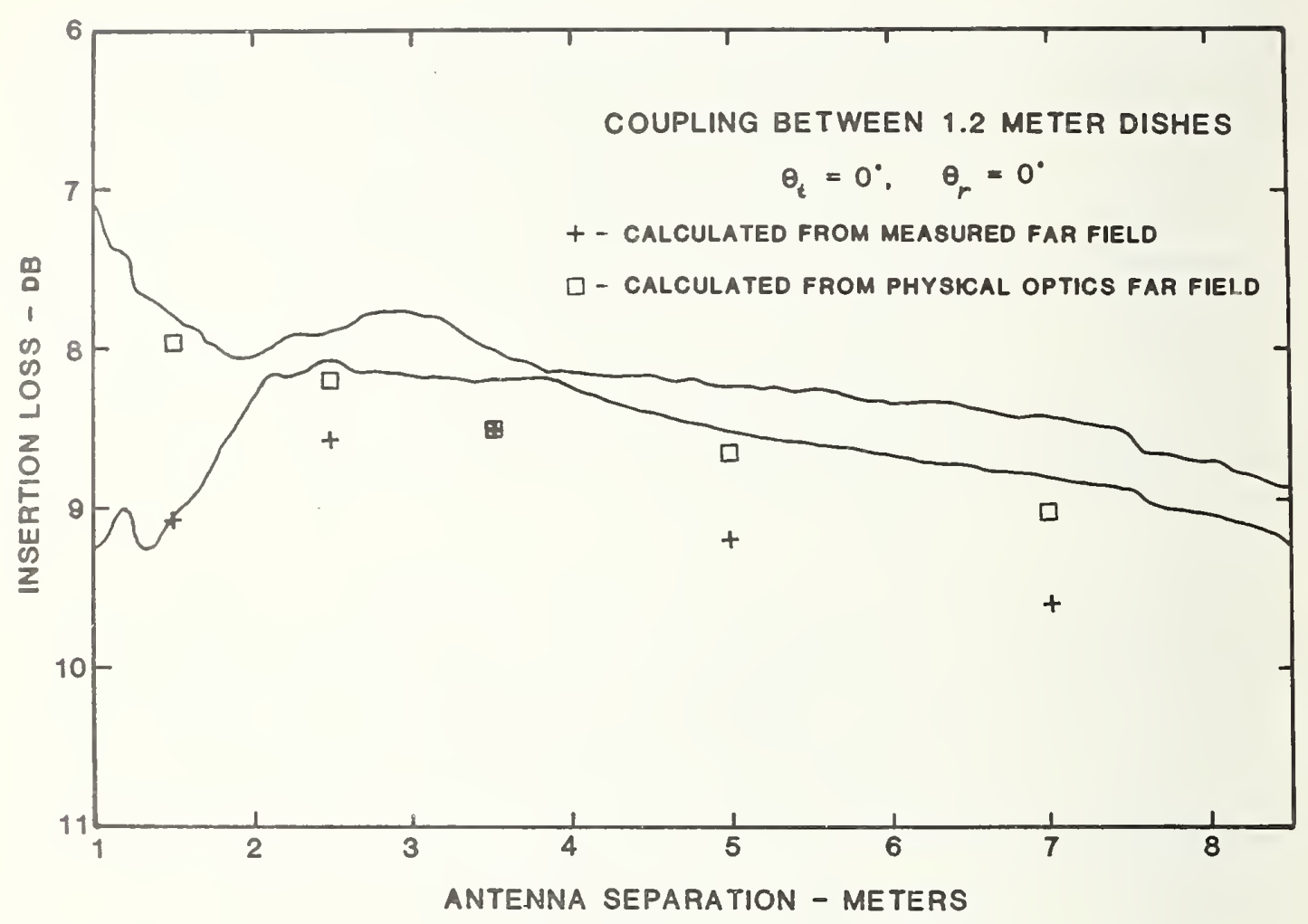

Figure 21. Mutual coupling between 1.2 meter reflector antennas.

Case 1: $\theta_{r}=0^{\circ}, \theta_{t}=0^{\circ}$. Solid lines indicate envelope of measured mutual coupling. 


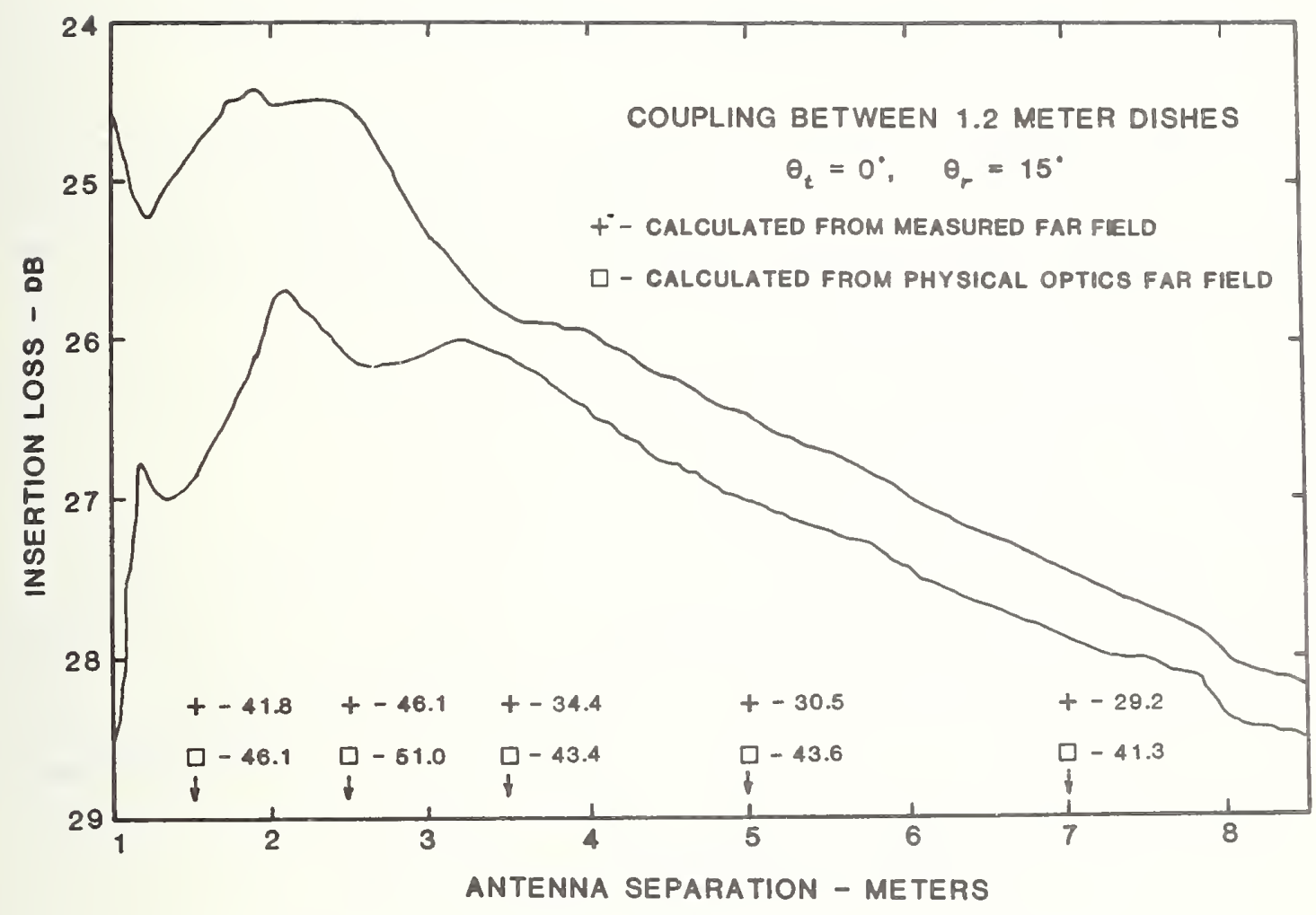

Figure 22. Mutual coupling between 1.2 meter reflector antennas. Case 2: $\theta_{r}=15^{\circ}, \theta_{t}=0^{\circ}$. Solid lines indicate envelope of measured mutual coupling. 


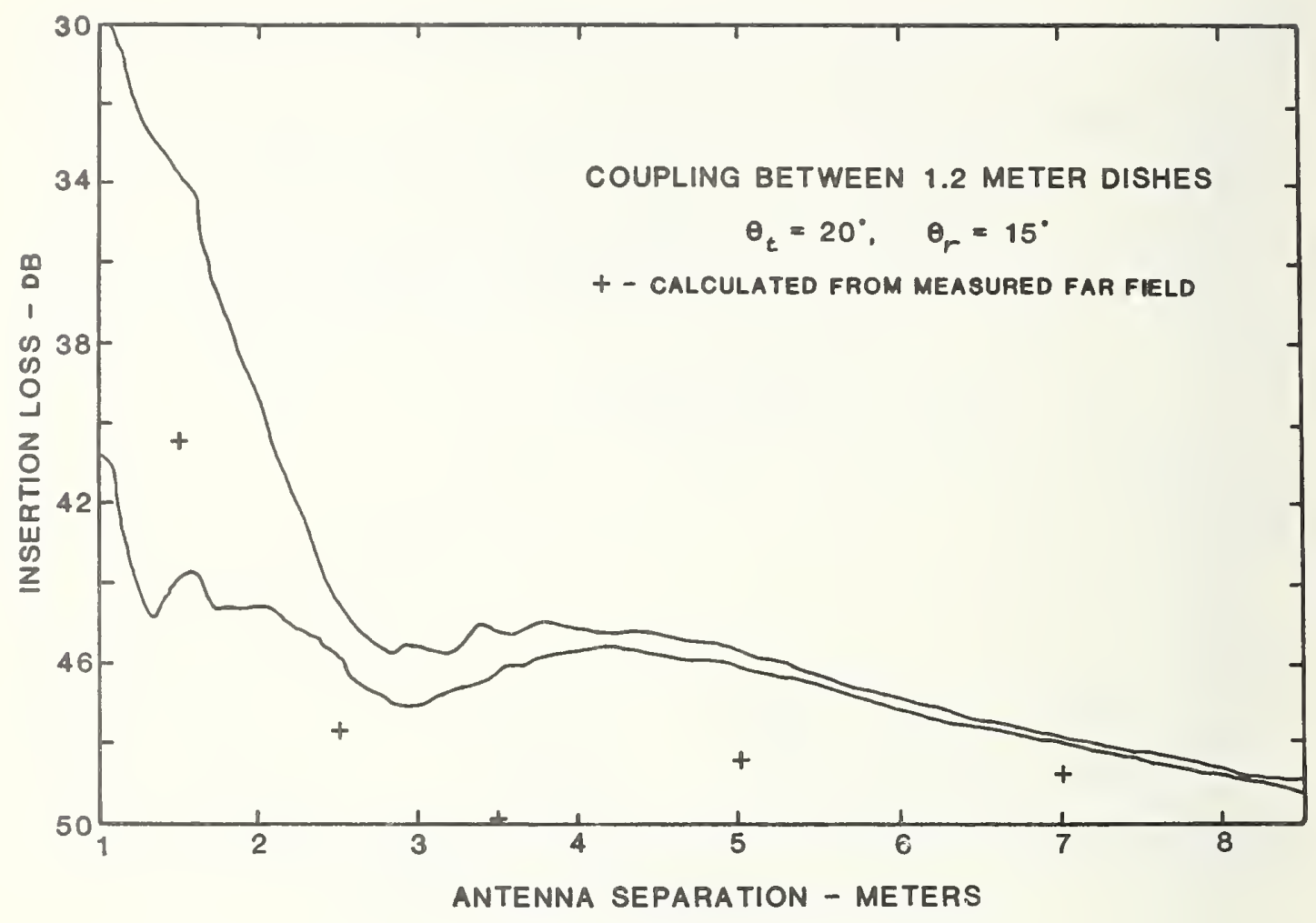

Figure 23. Mutual coupling between 1.2 meter reflector antennas. Case 3: $\theta_{r}=15^{\circ}, \theta_{t}=20^{\circ}$. Solid lines indicate envelope of measured mutual coupling. 
Several areas would appear to be worth pursuing. Certainly better models can be obtained. For the types of data required (complete two-dimensional, far-field patterns), a two-dimensional integration PO model is probably not practical. For this type of approach each far-field point would require a two-dimensional rather than a one-dimensional numerical integration. Further, because no symmetry is assumed, all needed far-field points must be computed rather than only the $\mathrm{E}$ - and $\mathrm{H}$-plane cuts. Because of these considerations, the computation of the complete pattern by a model which requires two-dimensional integration appears to be impractical. An alternative would be to calculate the main beam and first few sidelobes with PO, and use a GTD analysis for points farther off axis. Such a combination would use the best features of each technique.

A second alternative would be to reformulate the PO model in terms of aperture fields rather than surface currents. This approach would allow efficient computations using the FFT.

Contrasted with the above is the question of whether application might permit the use of less sophisticated models which would give upper-bound values for the desired quantities. Note that regardless of the sophistication of the model employed, certain antennas of a given type may fail to perform as predicted because of unit-to-unit variations. These variations have been observed to be as large as the discrepancies observed between measured and modeled fields for certain types of antennas.

With this in mind, we suggest two alternatives to the use of a sophisicated model. First, a catalog of measured far fields for antenna types in use could be compiled and these data used in coupling or near-field calculation. It would probably be necessary to measure several samples in order to determine expected unit-to-unit variations. An alternate approach would be to employ an envelope type of far-field pattern, such as the amplitude pattern that the CCIR recommended (32-25 $\log \theta$ function), if a reasonable phase function is also included.

It is recommended that these approaches be studied to determine if, in fact, they can give useful results.

\section{ACKNOWLEDGMENT}

The physical optics computer program was supplied by Prof. W. V. T. Rusch of the University of Southern California. Near-field measurements were performed by Mr. D. P. Kremer, who also assisted in the mutual coupling measurements. Helpful discussions with Dr. R. C. Baird, Mr. A. C. Newe11, and Prof. Rusch are also acknowledged. 


\section{REFERENCES}

[1a] Kerns, D. M., and Dayhoff, E. S., Theory of diffraction in microwave interferometry, J. Res. Nat. Bur. Stand., 64B, 1-13 (Jan. -March 1960).

[1b] Kerns, D. M., Plane-wave scattering-matrix theory of antennas and antenna-antenna interactions: Formulation and applications, J. Res. Nat. Bur. Stand, 80B, 5-51 (Jan.-March 1976).

[2] Yaghjian, A. D., The reactive and far-field boundaries for arbitrary antennas derived from their quality factor, National Radio Science Meeting (USNC/URSI), University of Colorado at Boulder (Jan. 9-13, 1978).

[3] Ames, J. S., and Murnaghan, F. D., Theoretical Mechanics, Ch. II (Ginn, Boston, Massachusetts, 1929).

[4] See, e.g., Cathey, W.T., Optica] Information Processing and Holography, Ch. 2 (John Wiley \& Sons, Inc., New York, N.Y.. 1974).

[5] See, e.g., the June 1967 issue of the IEEE Trans. on Audio and Electroacoustics.

[6] See, e.g., Johnson, C. C., Field and Wave Electrodynamics, section 10.5 (McGraw-Hill, New York, N.Y., 1965).

[7] Hu, Ming-Kuei, Near zone power transmission formulas, IRE Convention Record, $\underline{6}$, Pt. 8, 128-138 (1958).

[8] Rusch, W. V. T., Reflector antennas, in Numerical and Asymptotic Techniques in Electromagnetics, R. Mittra, Ed. (Springer-Verlag, New York, N.Y., 1975).

[9] Rusch, W. V. T., Course notes for short course, Reflector Antenna Theory and Design; University of Southern California, Los Angeles, California (July 1976).

[10] Tai, C-T, Dyadic Green's Functions in Electromagnetic Theory (Intext, New York, N.Y., 1972). 


\section{APPENDIX A. POMODL - PHYSICAL OPTICS ANTENNA MODEL}

This appendix includes detailed documentation of the program which models reflector antennas using physical optics and, at the user's option, calculates a two-dimensional far-field pattern for use by CUPLNF and also calculates near-field patterns on a specified plane. Each subroutine is documented individualiy, except for those which were obtained from other institutions and used unaltered, in which case only a brief description and listing is included. The final section of the appendix includes a sample input deck and a sample program output.

\section{A.1 GENERAL OVERVIEW OF COMPUTER PROGRAM}

The program POMODL and its associated subroutines are described in detail in the following subsections. The flow chart below is presented in order to give the reader an overview of the operation of the program package. 


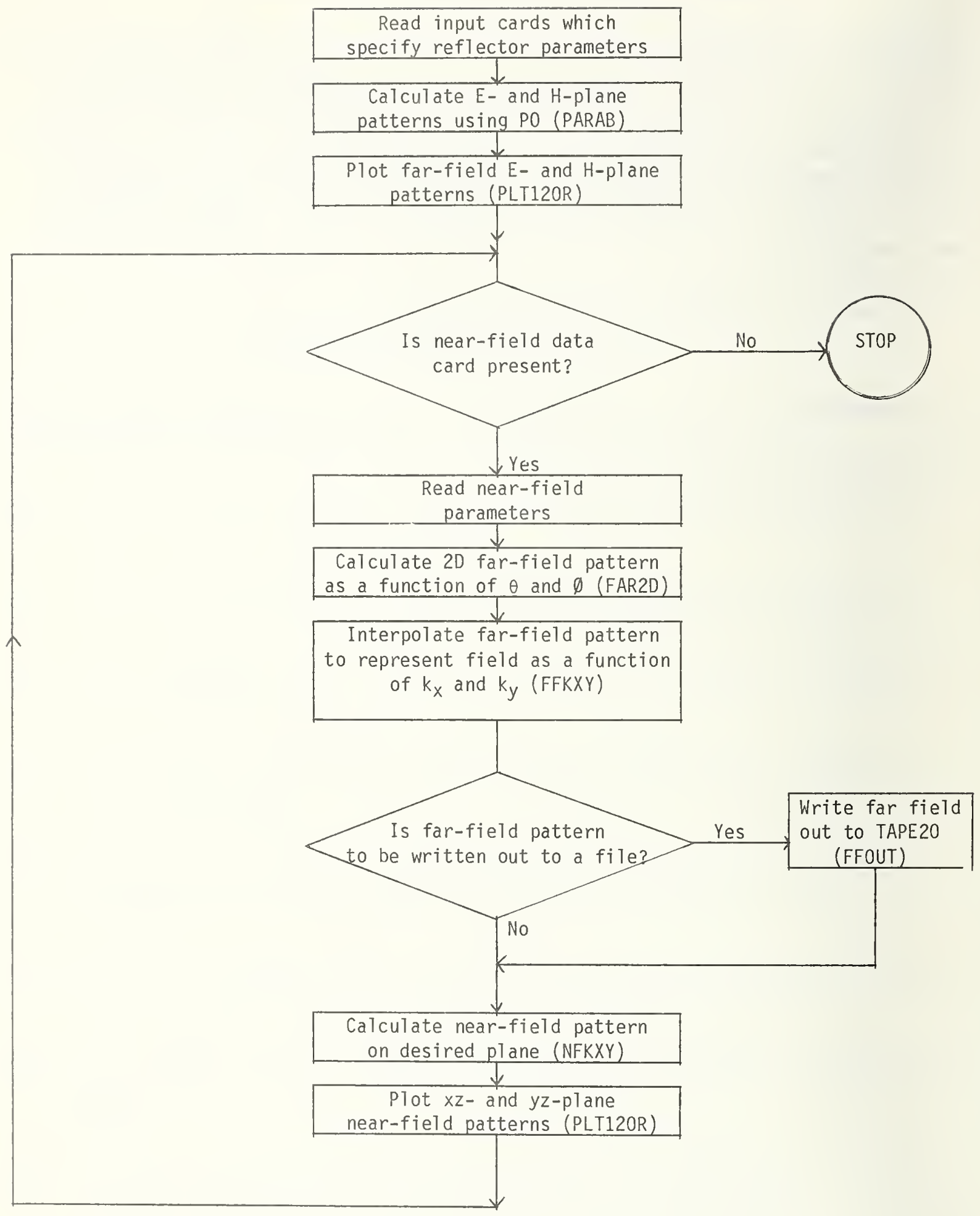




\section{A.1.1 PROGRAM POMODL}

PURPOSE:

To control input, output and flow of far-field calculation and transformation to near field.

\section{GENERAL DISCUSSION:}

This subroutine is a modified and extended version of SUBROUTINE PDRIVE written by Professor W. V. T. Rusch of the University of Southern California (USC). This subroutine reads data cards which specify the physical parameters of a paraboloidal reflector antenna and the parameters of the desired near-field patterns. It is basically a driver program for the USC PO subroutine PARAB and the subroutines which perform the far- to near-zone transformation.

The program produces plots and tables for far field in the E- and H-planes and nearfield cuts on a plane or planes perpendicular to the axis of symmetry of the reflector. In addition, the program calculates the near field on the complete plane and stores it in an array. This data may be obtained by a minor program modification. The far field presented in a table of values at equally spaced increments in $\left(k_{x}, k_{y}\right)$ space is also available at the user's option.

Because the techniques used require a substantial amount of computer core, it is recommended that the DIMENSION and COMMON statements specifying the size of arrays EY and DATAX be changed to suit the problem considered. Minimum size for EY is $2 \times$ (number of points to be calculated in $\theta$-direction) $x$ (number of points in $\emptyset$ direction). For DATAX, the size must be at least $2 \times$ (number of near-field points in $x$-direction) $x$ (number of near-field in $y$-direction). Because arrays EY and DATAX are not directly used by the main program but are dimensioned only for storage allocation purposes, they may be dimensioned as single dimensioned arrays whose sizes are greater than or equal to the values specified above.

\section{INPUT CARDS}

The input card deck consists of two groups of cards. The first five cards must be included in every run and specify the parameters of the antenna being modeled and the ranges and increments for the far field.

The second group of cards specifies the desired parameters of the near field to be calculated. If no cards of this group are present (i.e. only five input cards), only the E- and H-plane far-field patterns will be calculated and plotted. The near-field 
parameters are specified by a single card. Near fields for planes lying at different z-distances can be calculated by including multiple cards.

In addition, it is possible to specify that the far-field array which is calculated at evenly spaced points in $\left(k_{x}, k_{y}\right)$, space may be written out to logical unit 20 for use as input data for the mutual coupling program CUPLNF.

The following is a list and description of the data cards.

\section{Group I}

Card 1 Col. 1-40 This card contains alphanumeric information, usually the name and telephone extension of the person submitting the job.

Card 2 Col. 1-80 An alphanumeric identifier which is used to identify the case being studied. It appears as headings of tables and plots and on identification records of output files.

Card 3

This card specifies antenna parameters. All numbers on this card must have the decimal point explicitly specified.

Co1. 1-10 FOD - the F/D ratio for the reflector.

Co1. 11-20 FOL - the diameter in wavelengths of the reflector.

Co1. 21-30 BLOCK - the feed blockage as a fraction of the reflector diameter.

Co1. 31-40 DFOCUS - amount of axial defocussing in wavelengths, positive defocussing if the feed is beyond the focal point.

Col. 41-50 ACOSE-E-plane illumination factor.

If $A C O S E<-100$. aperture is uniformly illuminated.

-100. $\leq$ ACOSE < 0 . feed is a y-directed electric dipole.

ACOSE $\geq 0 . E-p l a n e$ feed pattern is $\cos \operatorname{ACOSE}(\pi-\theta)$ 
Col. $51-60$

Col. $61-70$

Card 4

Card 5

Group II

Card 6
ACOSH - H-plane illumination factor.

If $\mathrm{ACOSH} \geq 0$. $\mathrm{H}-\mathrm{pl}$ ane feed pattern is $\cos A C O S H(\pi-\theta)$.

FREQ - frequency in $\mathrm{GHz}$.

This card specifies parameters related to the far-field pattern calculated from PO. Except as noted, decimal points must be explicitly specified.

Col. 1-10 THETHF - initial value of theta - degrees.

Co1. 11-20 DTHETA - theta increment - degrees.

Col. 21-30 PHIF - initial value of phi - degrees.

Col 31-40 DLPH - phi increment - degrees.

Co1. 41-45 NTHETA - number of theta points desired, no decimal point, right justified in field.

This card gives data which allow calculation of magnitude of near electric field.

Col. 1-10 PIN - power input to antenna, a blank in field gives default value of 1.0 watt.

Co1. 11-20 EFF - assumed aperture efficiency of antenna in percent, a blank in field gives default value of 100 percent.

,This card specifies the parameters of the near field which is to be calculated. This card may be repeated to calculate near fields on different planes. If card 6 is omitted, only a far field will be computed and plotted.

Co1. 1-10 DELX - near field $x$-increment in meters.

Co1. 11-20 DELY - near-field $y$-increment in meters. 


\begin{abstract}
Co1. 21-30 DIST - distance from focal point of antenna reflector to near-field plane in meters.

Col. 31-40 Blank - field not used.

Co1. 41-45 IR2TON - number of y points desired in near field, no decimal point specified, right justified in field.

Co1. 46-50 IC2TON - number of $x$ points desired in near field, no decimal point specified, right justified in field.
\end{abstract}

\title{
OUTPUT
}

A copy of typical output for the program is included in section A.2. A table of input parameters is given first followed by the E- and H-plane far-field patterns for the antenna. Page printer plots for the E- and H-plane are then included.

The near-field parameters are then printed in a table giving the $x$ - and $y$-near-field centerline cuts. Finally, the amplitude and phase of the near-field centerline cuts are plotted.

\section{SYMBOL DICTIONARY:}

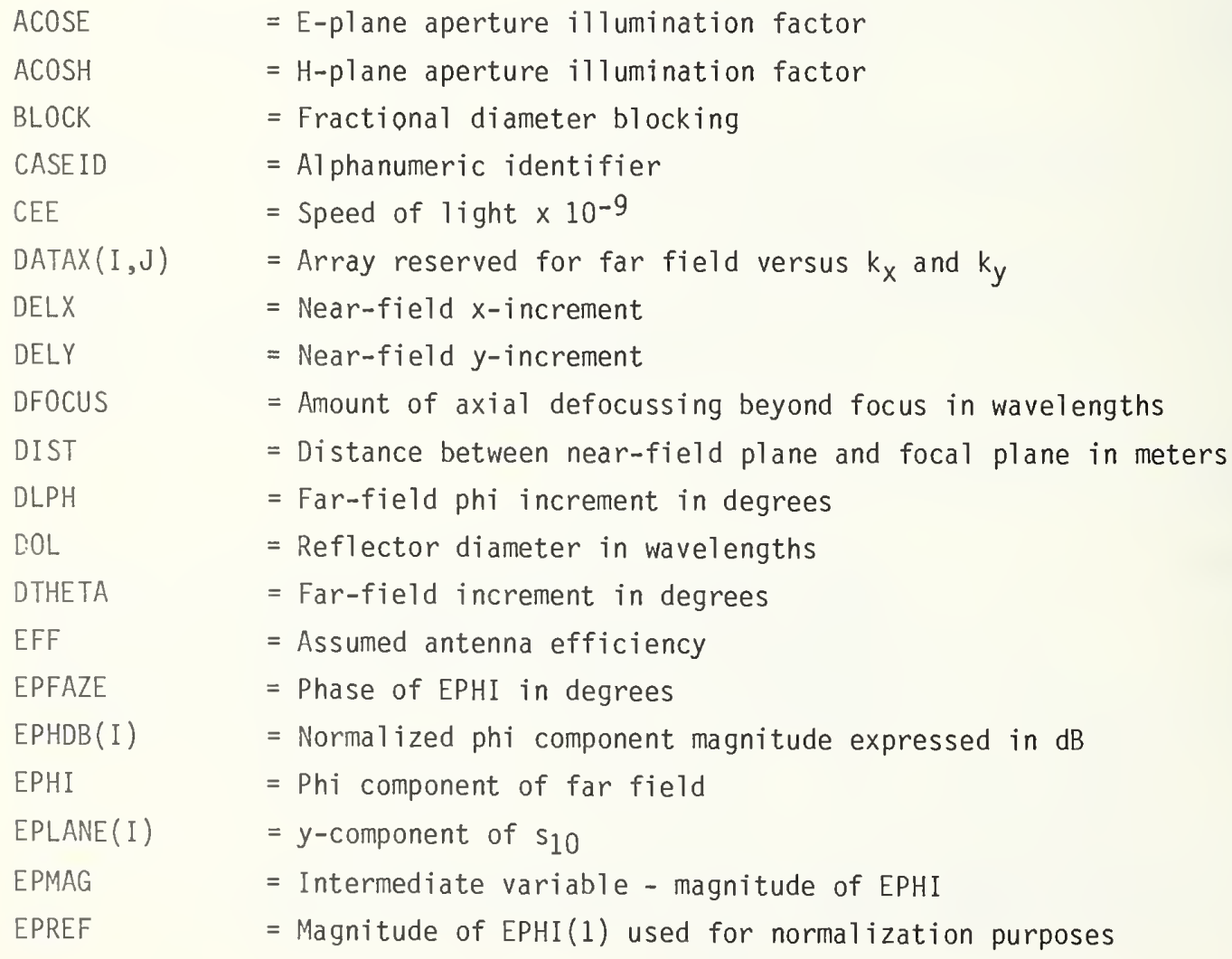




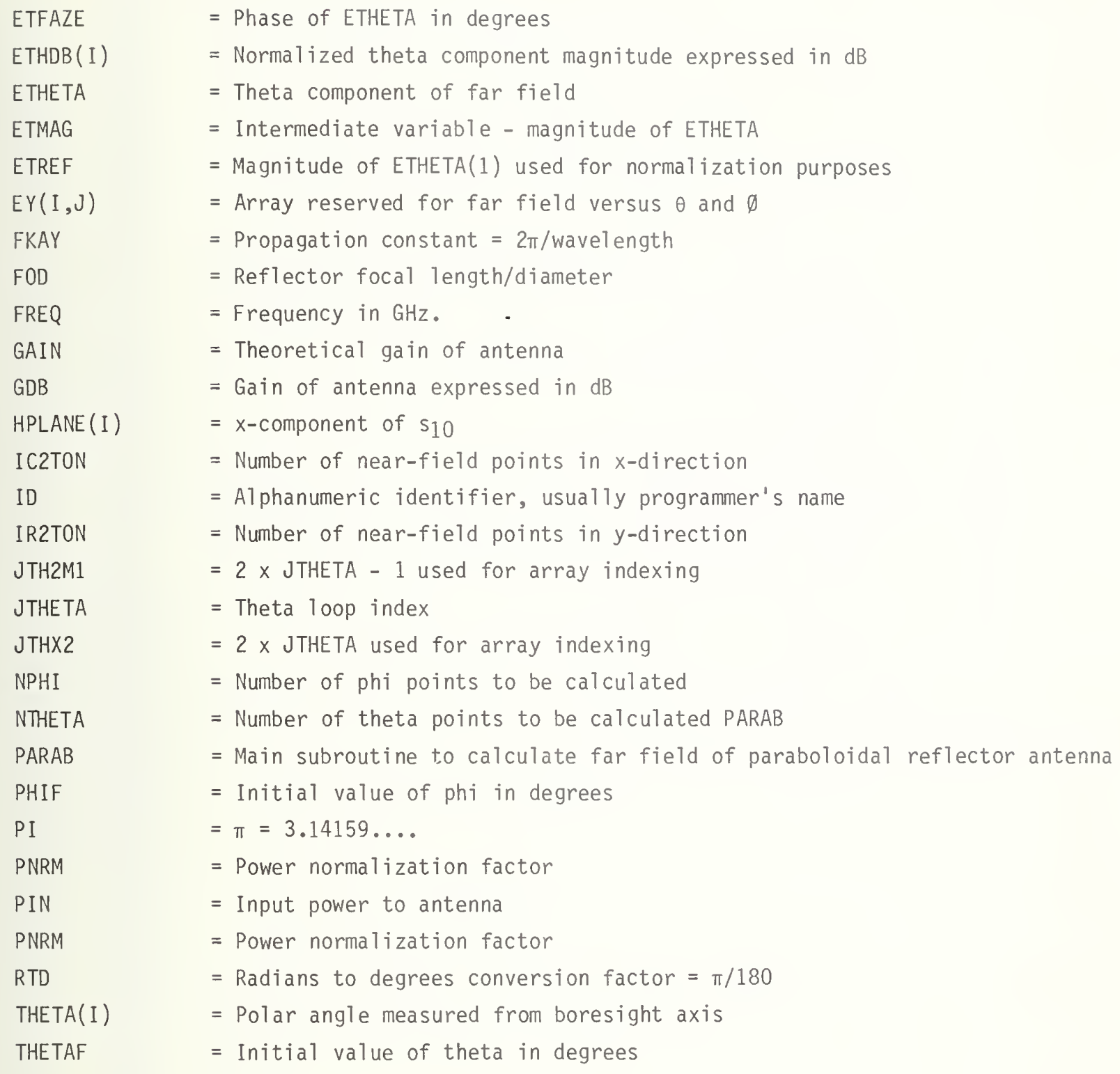

COMMON BLOCKS:

The labeled common used in POMODL is described below with a list of routines in which it is used. The variables are defined in the symbol dictionary.

COMMON /CNTRL/ DTHETA, DLPH, DELX, DELY, FREQ, DIST, PNRM

Routines using /CNTRL/: POMODL, FAR2D, FFKXY, NFKXY 


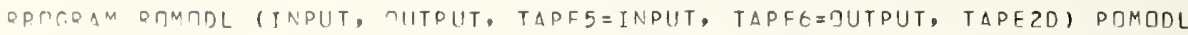

CDIVFQ DQCOPAY FQQ PFYSICAL OPTICS SUAPRUTINE PAPAB, WRITTFN BY PDIFFSSTQ W. V. T. RUSCH TF THE UNIVEPSITY OF SOUTHFRN CALIFIRNIA, WHICF INCLUDES CAPABILITY DF CALCULATING NFAP FIELDS IN A SPEC.IFIEN PLANF.

7-AXTC IS AXIS OF SYMNETRY DCINTING AWAY FPOM PARARMLOID. X IS PTLAD ANGLE THETA-PRIME MEASUPED FROM THE POSITIVE-T AXIS. $x=$ DI IS THF CIRFCTIMN TF THE REFLECTCR VFPTEX.

$X Q$ IS THF PDLAR ANGLE THFTA-DOUBLE-PPIME MEASIIPED FROM THE RCSITIVF T-AXIS WTTH THE CFFOCUSFD FFFO AS OPIGIN.

THE FTFLOS TF THE FEE $\triangle$ PE THF FIELES DF A CIPCULAR APEPTUQF. FXCITFO IN THF M=I $\triangle 7$ IMUTHAL MODE. THF F-PLANE IS THE YZ-PLANE ANT THE F-PLANE IS THF XT-PLANE. THE CDMPLEX ROLAR PATTERNS AI(TP) AND DI(TP) ARE SUCH THAT MOST DF THE PJWER IS DAOI $\triangle T E O$ TOWAFC. THE PFFLECTOD $\triangle N O$ VFQY LITTLE PDWER IS QAOTATFD INTI THF HALF-SPACF XP.LT.PI/?. FURTHEPMOPF, TO ASSIJDE CONTINLITY DF THE FIFLD WHEN XP $I P I$, IT IS NECESSARY $T H A T D I(P I)=-A I(P I)$.

$F \Gamma O=$ OFFLFCTRQ $F / \cap$

$\cap C L=D F F L F C T \cap R$ กIAMFTFQ IN WAVFLFNGTHS

BLOCK = FOACTIONAL DIA ETER BLCCKING POMODL POMODL PQMODL POMODL PIMODL $P \cap M O D L$ POMODL POMODL POMODL POMCDL PחNOOL POMODL POMODL POMODL POMOOL POMODL PDMDDL $P \cap M \cap D L$ POMODL PONCDL POMODL POMODL PCMODL POMODL MFPCUS = $\triangle M D U N T$ TF $\triangle X T A L$ DFFOCUSING BEYOND THE FICUS IN WAVELFNGTHPOMODL IF( $\triangle C \cap S F . L T \cdot(-1 C C . D))$ THF $\triangle P F R T I I R F$ IS UNIFORMLY ILLUMINATED IF(ACDSF.GE. (-100.0).AND.LT.D.D) THF FEFD IS Y-DIPECTED ELECTRIC POMODL CTOMLF

$[F(A P \cap S F . G F .0 .0) \Delta I=(C D S(P I-X P)) * \triangle C O S F, \times P . G E . P I / 2$ $=C, X P \cdot L T \cdot P I / 2$

$D I=-(C \cap S(P I-C P)) * \triangle C O S H, \times P, C E, P I / ?$ $=D, X P \cdot L T \cdot D T / ?$

$F Q F G=F Q F Q 1 J E N C Y$

THFT $\triangle F=$ INITTAL VALUE DF THFT $\triangle$, NEGRFES

OTHFTA = DIFFFPENTIAL VALUE COF THETA

DHIF $=$ INITIAL VALUF $T F$ DHI

DLPH = DIFFFPFNTIAL VALUF NF PHI

NTHFTA $=$ NUMBFR TF THETA VALUFS

PTN = DMWER INPUT TR ANTFNNA FOP NFAP-ZTNF FIFLC STRENGTH

FFF $=\triangle O F R T I M F$ FFFICIENCY RF ANTENNA

C.PNOLFX FTHFTA, EPHI

DIMENSICA FTHCA (2DD), FPHDB (2CO), THETA( $C \mathrm{CO})$

DIMENSITN EQLANE(4DO), HPLANF(4CO)

DIMENSTRN ID (4)

CEMMON EY(SCOO), DATAX(8192)

CTMMON /IDI CASFIR(R)

CIMMON /CNTRL/ MTHFTA, DLPH, DFLX, DELY, FRFO, DIST, PNRM

INPUT

PEAT $(5,5000)$ In

PQINT GOOI, ID

PFAD(5,5OOก) CASFID

DDMDOL

ROMODL

POMODL

POMODL

POMODL

PONODL

ROMODL

POMODL

POMODL

POMODL

POMODL

POMODL

POMODL

PIMODL

POMODL

PONODL

POMOOL

POMODL

POMOOL

POMODL

POMODL

POMODL

PDMODL

POMODL

POMODL

POMODL

POMODL

PCMODL

POMODL

POMODL

POMODL

WRITF $(5,6000)(\triangle A S E I D$

PFAD (5,5020)FCD, DCL, BLDCK, DFOCUS, ACPSF, ACOSH, FREQ

POMODL

PFAD (5, 5040) THFTAF, DTHFTA, PHIF, DLPH, NTHFTA

POMODL

PFAF( $5,50>0)$ RIN, FFF

IF (PIN. FG. O.) PIN $=1 . \mathrm{C}$

IF (FFF. FD. O.) EFF $=1$.

WRTTE $(h, 202 D) F C O, D C L$, RLOCK, DFOCUS, FRFO

IF ( $\triangle C D S E \cdot L T \cdot(-1 C \Gamma \cdot C))$ WITE $(6,6 C D 5)$

IF( $\triangle C \cap S E . G F .(-100.0) . \triangle N \cap . \triangle C M S F . L T .0 .0) W P I T F(6,6 C 1 D)$

IF (ACDSF. SF. 0.0)WRITF $(6,6015) \triangle C O S F, \triangle C C S H$

POMODL

POMODL

POMODL

PCNODL

POMODL

POMODL

PCMODL

POMODL

MISCFLLANEDUS

$P I=4.0 * \Delta T \Delta N(1.0)$

PTD $=180.01 \mathrm{PI}$

CFF $=.2097975$

PONONL

PDMODL

POMODL

POMODL

POMODL

POMCDL 
$F D R=10 * A L D G I O(G A I A)$

PNRM = SQRT (15.*RIN*CAIN/FKAY/FKAY)/PI

PDNODL

POMCDL

WRITF( GD, GD2) EFF辛ICC, GDB, PIN

PCM.CDL

ROMOOL

POMCDL

WRITF(h, h030)

ENTER THE THETA LECP

PTMTOL

POMODL

NC 10D JTHETA $=1$, NTHETA

PTMODL

PCNODL

THETA $(J T H E T A)$ THETAE + (JTHETA-1)*DTHETA

POMOOL

ETMAG = CARS (ETHETA)

IF(JTHETA. OD.1) FTREF = ETMAG

FTHDR (JTHETA) $=20.0$ ALOG10 (FTMAG/ETREF)

ETFAZE = - $\triangle T A N Z(A I M A C(E T H E T A), R E A L(E T H E T A)) * D T D$

FPMAG = CARS ( $F R H T)$

IF (JTHETA.EO.1) FRREF = ERM $\triangle E$

EPHDB (JTHETA) = 20.D*ALDGIO (EPMAG/EPREF)

EPFAZF = - ATAN2(AIMAF(EPHI), RFAL (EPHI))*RTD

PRINT A040, THETA (JTFFTA), ETMAS, ETHDB(JTHETA), ETFAZF, EPMAG,

IFPHDR (JTHETA), EDEAZE

JTHX? = JTHETA*2

$J T H M 1=J T H \times 2-1$

NCRMALIZF E TI 1. C AT THETA $=0$. ANO CALCULATE S10.

ER: ANF(JTH2M1) = CABS(FTHFTA)/ETREF/COS(THETA (JTHFTA $/ R T O$

FDL $\triangle N F(J T H X \geq)=F T F A T E / R T D$

HPLANF(JTH2M1) = CAFS (FRHT)/FTDFF/CCS(THFTA (JTHETA)/RTD)

HPLANE(JTHXZ) = EPFAZE/RTO CINTINIIF

PLOI E AND H-PLANE AMPLITUDES

PCMODL

PQMCDL

POMOOL

PCMCOL

POMODL

PCMCOL

PПMПOL

PחMCDL

PCMDOL

PCMCOL

POMCDL

POMODL

ROMCDL

PONOOL

PCMDDL

PCMOOL

POMOOL

POMOOL

PCMOOL

PCMNDL

PCMOOL

PCNOOL

POMONL

PCMODL

PCMCOL

CALI. PIT12DP(THFTA, ETHDR, HC, - $50 ., 0 .,-50 .$, NTHFTA, 1 H*, 1,1$) P C M M O L$ PRINT SOAO, CASEID, 1 CH E-PLAMIE

PCMODL

CALL RLT1?OR (THFTA, FPHDR, 60., -60, $0 \ldots,-50$, NTHFTA, 1H., 1, 1)PDMOOL PRINT SCBO, CASEIT, ICH HOPLANE

POMODL

$P C M O O L$

$P \cap M O D L$

READ TATA FTR NFAQ FIELD

OFLX = NFAD FIELO X-SRACING

NELY $=$ NFAR FIFLD Y-SPACING

CIST = Z-POSITICN CF NEAR FIFLD PLANE. (DIST = 0. IS FOCAL DLANE TE PAPARTLA)

MHMMY - NMT CUDPFNTLY USFO

ID?TCN = NUMREP CF POINTS IN Y-AODAY

IC $2 T \cap N=$ NUMRER DF PחINTS IN X-ARPAY

1 RFAD 5ח4O, OELX, DELY, DIST, DUMMY, IPZTCN, ICZTON IF $(F \cap F(5)) 20 n$, ?

2 RRINT 4O73, OELX*100., IC?TDN, DELY*100., IR2TIN, DIST*10D. C.ALL FAD?O IFPLANE, FPLANE, EY, NTHETA, NPHI, OATAX, IRZTCN*2, $\operatorname{II} 2 \operatorname{THN}$ Gr Tח 1

ENCC F[RNAT(RAID)

POMOOL

DOMOOL

PחMODL

PONODL

PONODL

RCMDOL

PกMDDL

POMODL

PINOOL

POMOOL

POMODL

RDMDOL

PCMOOL

PCMCDL

PDMOOL

$P \cap M \cap O L$

RDMOOL

PIMOOL

$P C M O D L$

PTNOOL

$50 ? 0$ FTRMAT(\&F10.0)

140

FTDNAT (4FIO.C, 2 I 5)

PDNCDL

\#\#, II, T7, Q AIC)

4001 F[PNAT(1H, a $A 10)$

SCO? FOOMAT IT16, \#ASCIJMEC FFFICIENCY = *, FI0.2, * PERCENT*, /,

RCMCDL

PTM DDL

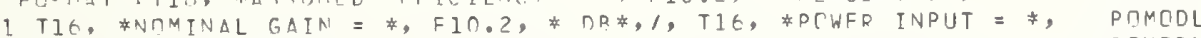

145

2 FIr.?, \# WATTS*, / /

$P C M D O L$

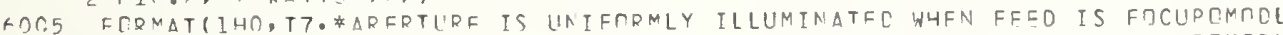
*SFD*, 1)

DIMCOL

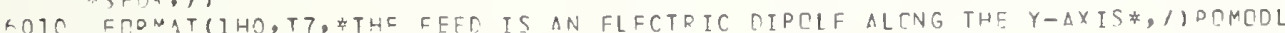

6015 FCPMAT(IHD,T7, XFFFD F-PIANE PATTFRN $=(\operatorname{COS}(Y)) \neq(\neq, F 5.2, \neq) \neq, 1, \quad P C M C D 1$ *T 12, $\neq H-P L A N F$ PATTFDN $\left.=-(C \cap S(Y)) * \frac{*}{*}(\neq, F 5,2, \neq) \neq, 1\right)$ PIMODL

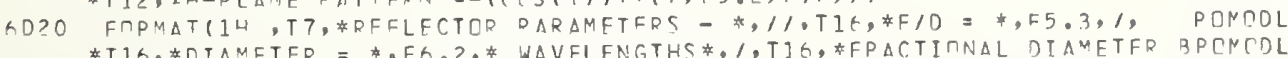

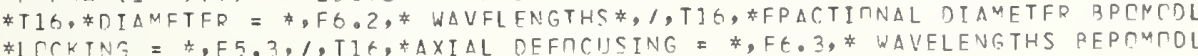




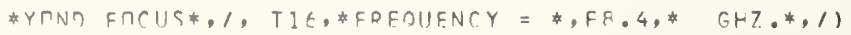

6030 F[RMATI ] HO, T30, \#F-PLANF\#,T62, *H-PLANE*, I,

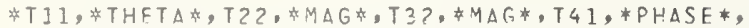

*T54, *MAG*, T64, \#MAG*,T73, *DHASF*, I,

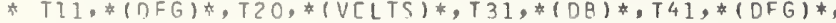

*T5?, * (VOLTS) * TE3, * (DR) *, T 7?, \#(DFG)*, /)

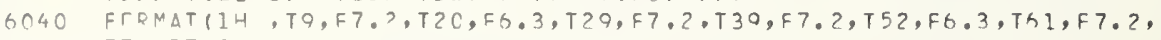
\& T T1, F 7.2)

EC60 FORMAT(///, * ECF FCUNC CN LU5*)

GCTO FDRMAT(//1, T7, *NEAR-FTELD PAPAMETFRS-\#, 1/,T1t, *X-SP $\triangle C I N G=*, \quad P O M C D L$

IFG.2, \# CM*. T5O, I6, \# PCINTS*, 1,TIE, *Y-SPACING =*, F6.?, \#CM\#, POMODL $2 T 50, I E$, PIINTS*, /, TIE, *DISTANCE FROM REFLECTOR FOCAL POINT = \#, DOMODL 2FR.2, * CM AWAY FRTM RFFLECTRQ SIIRFACE*,//I

FORO FIOMAT( $, 5 X, 8 A 1 C, E X, \triangle 10)$

END 


\section{A.1.2 SUBROUTINE FAR2D(EPL, HPL, EY, NTHETA, NPHI, DATAX, IR2X2, IC2TON)}

PURPOSE:

To produce a two dimensional array of far-field data given the E-and H-plane cuts for an axially symmetric antenna.

ARGUMENTS:

EPL is a complex vector containing the far-field, E-plane pattern of the antenna stored in amplitude phase form.

HPL is a complex vector containing the far-field, H-plane pattern of the antenna stored in amplitude-phase form.

EY is a complex array which contains a two-dimerisional, far-field array, arranged as a function of theta and phi.

NTHETA is the number of points in theta direction.

NPHI is the number of points in phi direction.

DATAX is not used in this subroutine, see FFKXY.

IR2X2 is twice the number of rows of data produced as a function of $k_{x}$ and $k_{y}$.

IC2TON is the number of columns of data in far-field array produced as a function of $k_{x}$ and $k_{y}$.

METHODS:

Circular symmetry is assumed in the antenna, hence, it is necessary to calculate the far-field pattern over one quadrant only. The subroutine calculates the main rectangular component of the far field which is given by

$$
E_{y}(\theta, \theta)=E_{y E}(\theta) \cos \theta \sin ^{2} \emptyset+E_{y H}(\theta) \cos ^{2} \emptyset
$$

where

$$
\begin{aligned}
& E_{y E}(\theta)=\text { Electric field in E-plane as a function of theta. } \\
& E_{y H}(\theta)=\text { Electric field in H-plane as a function of theta. }
\end{aligned}
$$

Under the assumption of circular symmetry, this subroutine calculates the far-field $y$ component as a function of $\theta$ and $\emptyset$ given the $E$ - and $H$-plane patterns $(\emptyset=0, \pi / 2)$ as a function of $\theta$.

This subroutine uses library functions: ATAN, COS, and SIN. 


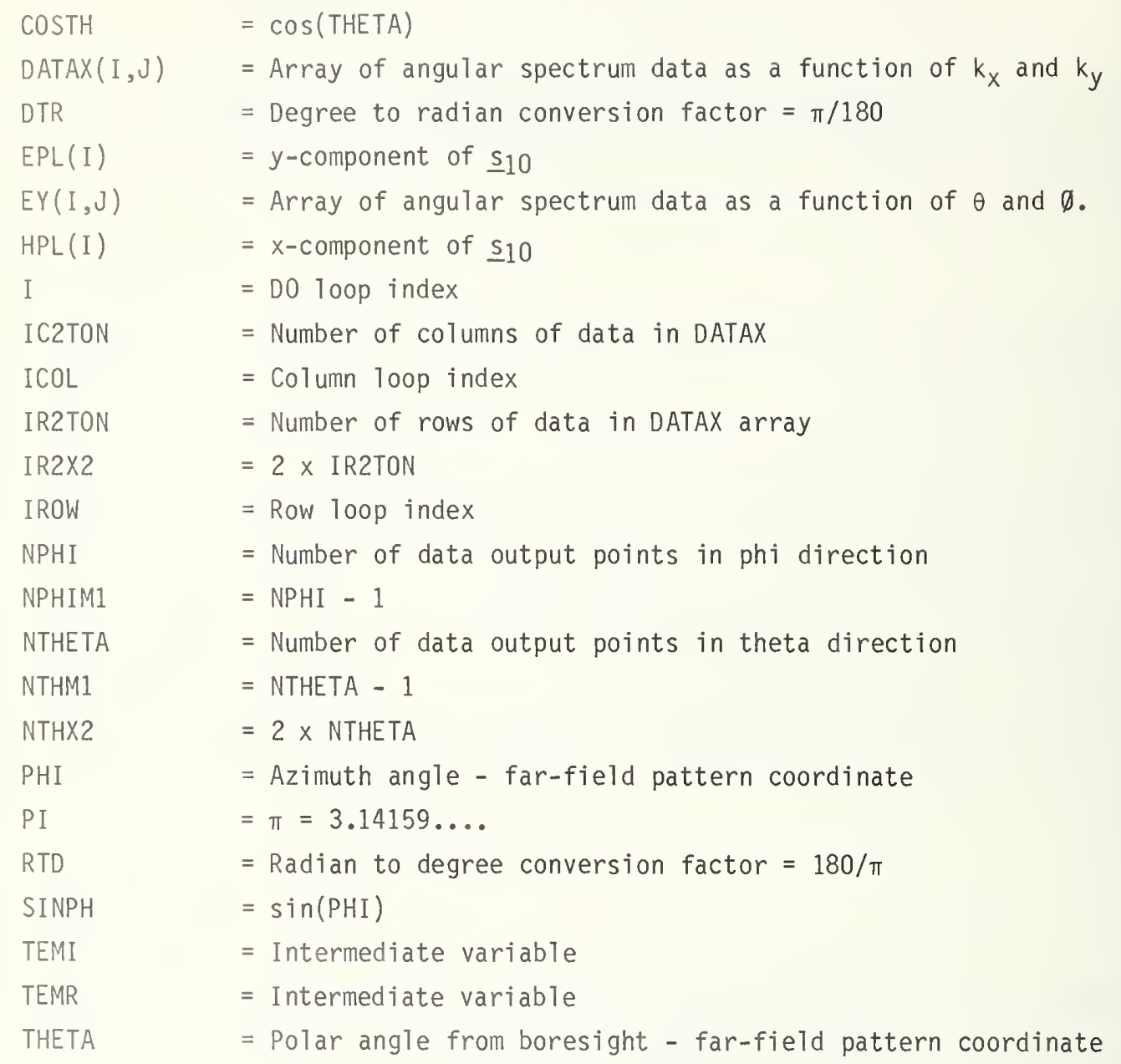


1

5

10

15

20

25

30

35

SURRTUTINF FAR2D(EPL, HPL, EY, NTHETA, NPHI, DATAX, IRZX2, IC 2TON)FAR 2D

C-

C-

c-

$\mathrm{C}-$

c-

C-

C-

C-

WHICH HAVF SEPARABLE FAR-FIELD PATTERNS.

DIMFNSIJN EDL(1), HPL (1), FY(NTHETA,NPHI), DATAX(IR2X2, IC RTON) COMMIN /CNTRL, OLTH, OLPH, DFLX, DFLY, FRFO, DIST, PNRM COMPLFX FY

$R I=4 * * A T A N(1$. NTH ${ }^{2}=2$ NTHETA
$F A R 2 D$

FAR 20

$F A R 20$

FAR 20

$F A R 2 D$

$F A R 2 D$

FAR 20

FAR 20

$F \triangle R 2 D$

FAR $2 D$

FAR 20

$F \triangle R 2 D$

FAR 20

$F \triangle R 20$

$F \triangle R 20$

$F \triangle R 2 D$

$F A R 20$

FAR 20

$F \triangle R 2 D$

$F A R 2 D$

$F A R \geq D$

FAR $2 D$

FAR 20

$F \triangle R ? D$

FAR $2 D$

FAR 20

$F A R ? D$

FAR $2 D$

FAR 20

FAR $2 D$

FAR 20

$F A R 20$

FAR 20

FAR 20

FAR $2 D$

FAR 20

FAR 20

$F \triangle R 20$ 
PURPOSE:

To produce an array of two-dimensional, far-field data which is equally spaced in the coordinates $k_{x}$ and $k_{y}$, given an array which is equally spaced in the coordinates $\theta$ and $\varphi$.

ARGUMENTS:

DATAY is a two-dimensional array of far-field values, expressed as a function of equally spaced $\theta$ and $\emptyset$ coordinates in the quadrant $0 \leq \emptyset \leq \pi / 2$. Complex far-field values are expressed with real and imaginary parts adjacent in storage, such. as FORTRAN IV stores them. Note, after execution, DATAY is expressed in polar form because of a call to ARAYRTP.

NTHX2 is twice the number of points in $\theta$ direction.

NPHI is the number of $\emptyset$ points in one quadrant.

DATAX is the output array of far-field points which are equally spaced in $k_{x}$ and $k_{y}$. Complex far-field values are expressed in polar form with amplitudes and phases stored in adjacent locations. This array contains far-field values of an entire hemisphere rather than a single quadrant as is the case for DATAY.

IR2X2 is twice the number of rows ( $k_{y}$ values) in the DATAX array.

IC2TON is the number of columns $\left(k_{x}\right.$ values) in the DATAX array.

\section{METHODS:}

FFKXY is basically an interpolation routine which fills each point in the DATAX array, by calculating the corresponding values of $\theta$ and $\emptyset$ locating the four nearest points corresponding to these values in the DATAY array. The value stored in DATAX is then a weighted average of these four points. The program assumes that the far-field input array is from a single quadrant such as produced by FAR2D, and produces a far-field output array over the entire hemisphere by reflecting about the lines $k_{x}=0$ and $k_{y}=0$.

Because the FFT is used to calculate the near-field distribution, it is necessary to have a far field which is sampled on equally spaced points in $k_{x}$ and $k_{y}$. Further, we chose the spacing so that the near-field spacing will satisfy the sampling theorem criteria. Thus, the far-field increments $k_{x}$ and $k_{y}$ are fundamentally related to 
the near-field spacing which is specified and transmitted into the subroutine via common CNTRL. Relationship between $k_{x}, k_{y}$, the far-field increment, and $\delta_{x}$, $\delta_{y}$, the near-field spacings, are,

$$
\Delta k_{x}=\frac{2 \pi}{\delta_{x} N x}, \Delta k_{y}=\frac{2 \pi}{\delta_{y}^{N} y}
$$

Beginning at the center $\left(k_{x}=k_{y}=0\right)$ of the DATAX array, the value of $\theta$ and $\emptyset$ corresponding to $k_{x}$ and $k_{y}$ are calculated. These are given by

$$
\begin{aligned}
\theta & =\cos ^{-1} \sqrt{1-k_{x}^{2} / k^{2}-k_{y}^{2} / k^{2}} \\
& =\tan ^{-1}\left(k_{y} / k_{x}\right)
\end{aligned}
$$

The indices corresponding to the four elements in the DATAY array that lie closest to the value of $\theta$ and $\emptyset$ are computed. A linear two-dimensional interpolation is then performed using these four points in order to compute the value desired. The interpolation is performed on the amplitude and phase, not on the real and imaginary parts of the DATAY array.

Care must be exercised in interpolating the phase, because the phase is only given modulo $360^{\circ}$. This causes errors in performing the interpolation when the phase function makes a jump between two points in question unless a correction is applied to one of the phases. In this subroutine, three of the four phases are reset to lie on the same cycle as the reference phase by testing to see that the absolute value of the phase difference between the point in question and the reference is less than $180^{\circ}$. This procedure is valid provided that the far-field data points are spaced closely enough. A reasonable requirement would be to have at least 4 or 5 far-field points in an angular range of a sidelobe, a requirement which is met anyway if a sufficiently smooth pattern is produced.

The interpolation is performed by taking a weighted average of the amplitude or adjusted phases of the form surrounding points, the weighting of an individual point being inversely proportional to its distance from the point in question.

\section{SYMBOL DICTIONARY:}

$\begin{array}{ll}\text { C(I) } & =\text { Coefficients used to calculate } k_{x} \text { and } k_{y} \text { from near-field spacing } \\ \text { CEE } & =\text { Speed of light } \times 10^{-9} \\ \text { D33J1 } & =\text { Intermediate variable used in phase test } \\ \text { D43J } & =\text { Intermediate variable used in phase test } \\ \text { D43J1 } & =\text { Intermediate variable used in phase test }\end{array}$




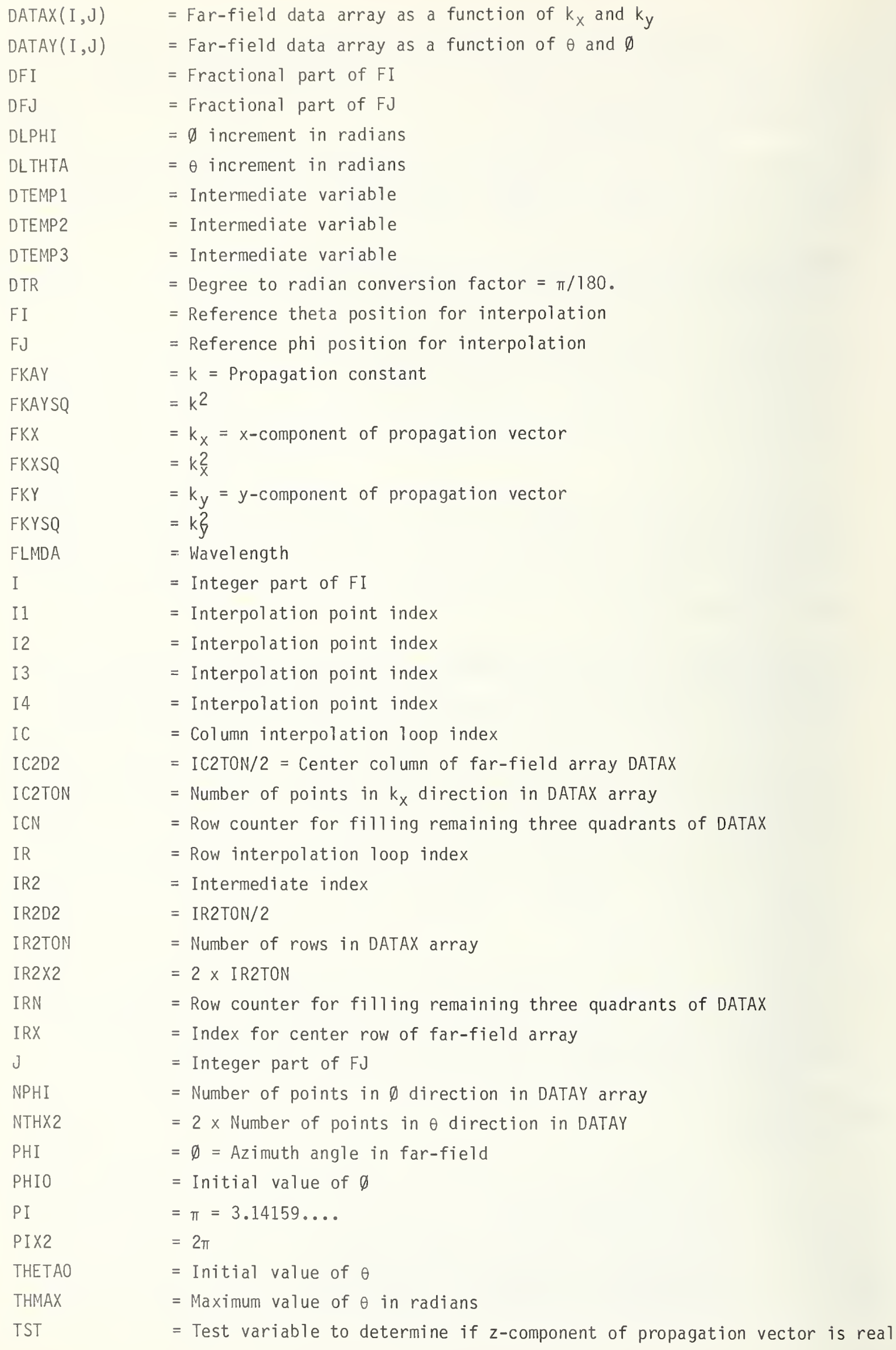

$=k_{x}=x$-component of propagation vector$$
=k_{x}^{2}
$$$$
=k_{y}=y \text {-component of propagation vector }
$$$$
=k^{2}
$$$$
\text { = Wavelength }
$$$$
\text { = Integer part of FI }
$$$$
\text { = Interpolation point index }
$$$$
\text { = Interpolation point index }
$$$$
\text { = Interpolation point index }
$$$$
\text { = Interpolation point index }
$$$$
\text { = Column interpolation loop index }
$$$$
\text { = IC2TON/2 = Center column of far-field array DATAX }
$$$$
=\text { Number of points in } k_{x} \text { direction in DATAX array }
$$$$
\text { = Row counter for filling remaining three quadrants of DATAX }
$$$$
\text { = Row interpolation loop index }
$$$$
\text { = Intermediate index }
$$$$
\text { = IR2TON/2 }
$$$$
=\text { Number of rows in DATAX array }
$$$$
=2 \times \text { IR2TON }
$$$$
\text { = Row counter for filling remaining three quadrants of DATAX }
$$$$
\text { = Index for center row of far-field array }
$$$$
\text { = Integer part of } \mathrm{FJ}
$$$$
=\text { Number of points in } \emptyset \text { direction in DATAY array }
$$$$
=2 \times \text { Number of points in } \theta \text { direction in DATAY }
$$$$
=\varnothing=\text { Azimuth angle in far-field }
$$$$
\text { = Initial value of } \emptyset
$$$$
=\pi=3.14159 \ldots \text {. }
$$$$
=2 \pi
$$$$
\text { = Initial value of } \theta
$$$$
\text { = Maximum value of } \theta \text { in radians }
$$$$
\text { = Test variable to determine if z-component of propagation vector is real }
$$ 
CTMMTN /CNTRL, DLTH, CLPH, DFLX, DFLY, FPFD, CIST, PNRM

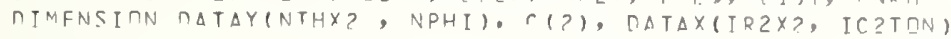

LIIIUT $=20$

IP $2 T \cap N=I R ? \times 21$ ?

$P I=4 . * \triangle T A N(1) \quad \$ P I X ?=$.$? ? P I$

$C F F=. ? 997925$ \& FLMIA $=$ CFF/FDFO

DTO = DIIIRO.

$F K A Y=D I \times 2 / F L M D A \quad F K \Delta Y S Q=F K \Delta Y * F K \Delta Y$

THFTO $=$ ?

THMAX $=(N T H X ? / 2-2)$ D T THTA

$r(I)=R[X>1(\cap F L X \pm I C ? T \cap N !)$

CALI. ARAYRTP(CATAY, NTHX?, NPHI)

$T C A !=0$

DC 61 IC $=$ IC 2D2, IC2TL

$F K X=C(1) \neq(1 C-I C .2[2)$

$I P N=0$

$O C$ OL IR = IRZLZ, IRZTON

IF ? $=[Q \$ 2-1$

$F K Y=(12) \notin(I D-I R 2 C ?) \quad$ क $\quad F K Y S Q=F K Y * F K Y$

$T S T=F K A Y S O-F K X S O-F K Y S O$

IF (TST, LT, O.) GC TC AO

THETA $=\Delta C$ OS ( $(S Q R T(F K \Delta Y S O-F K X S O-F K Y S Q)) / F K \Delta Y)$

IF (THFTA GT. THMAX) ED TD 80

IF (FKY. LT. O.) THFTA $=$-THFTA

IF (FKX .FO. C. . ANR. FKY.FO. C.) GOTC+3

PHI $=A T A N Z(F K Y, F K Y)$

40

GI Tח 44

62 PHI $=0$.

64 IF $(P H I \cdot L T \cdot 0) \quad P H I=.P H I+P I$

45

r- INTFORTLATF DATAY ADRAY TI PPROUCF CATAX AFPAY WHICH IS EOIJALLY

C- SPACEL IN KX AND KY.

$\mathrm{C}-$

C FIND THF INDICFS FIR THE INDIJT DATA WHICH IDFNTIFY THF CDORDINATES

C SLIOSEST TO THE DESIPED THETA AND PHI VALUES. INTFPPCLATE TO FINO THF

C DRTRF PATTFPN $\triangle T$ THF DFSIDFT POINT.

50 c

$F I=((T H E T \Delta-T H E T O) / D L T H T A)+1.0$

$F J=((P H I-R H I 0) / C(P H I)+.99999999$

IF(PHI. FO. O.) FJ $=1$.

$I=F I$

$\mathrm{J}=\mathrm{FJ}$

$D F I=F I-I$

OFJ $\mathrm{F}=\mathrm{FJ}-\mathrm{J}$

I $]=? * I-I$

$\mathrm{I} 2=\mathrm{I} 1+$ ?

I $3=?+I$

$[4=[\underline{x}+$ ?

$F K X Y$

FFKXY

FFKXY

FFKXY

FFKXY

FFKXY

FFKXY

FFKXY

FFKXY

FFKXY

FFKXY

FFKXY

FFKXY

FFKXY

FFKXY

FFKXY

FFKXY

FFKXY

FFKXY

FFKXY

FFKXY

FFKXY

FFKXY

FFKXY

FFKXY

FFKXY

FFKXY

FFKXY

FFKXY

FFKXY

FFKXY

FFKXY

FFKXY

FFKXY

FFKXY

FFKXY

FFKXY

FFKXY

FFKXY

FFKXY

FFKXY

FFKXY

FFKXY

FFKXY

FFKXY

FFKXY

FFKXY

FFKXY

FFKXY

FFKXY

FFKXY

FFKXY

FFKXY

FFKXY

FFKXY

FFKXY

FFKXY

FFKXY

$I D X=I R P \cap ? \div 2-1$

FFKXY

FFKXY

FFKXY

FFKXY

¿ DFTFRMINF $\triangle M P$ AT (THETA,PHI) BY WFIGHTEN AVER

FFKXY

FFKXY

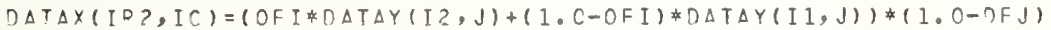

FFKXY

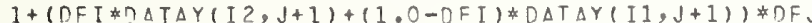

\section{$(I 3, J)$}

c

C RESFT PHASE AT (I4,J) IF NFCESSAPY SD THAT THF ABSOLUTE VALIJ DF T43J 
IF $(\cap 43 \mathrm{~J} \cdot G \mathrm{~T} \cdot 180 . \mathrm{C})$

$1 \Gamma T F M D I=\cap \triangle T \Delta Y(I 4, \mathrm{~J})-3 \in \mathrm{C} . \mathrm{C}$

IF $(\cap 43 \mathrm{~J} \cdot L F .180 .0 . \Delta N D .043 \mathrm{~J} \cdot G F \cdot-190.0)$

$1 \cap T E M D I=\cap \triangle T \triangle Y(I 4, \mathrm{~J})$

IF (n4 J J.LT. -190.0$)$

] กTFMP $1=D \triangle T A Y(I 4, J)+3 A \cap . \Omega$

$r$ RFSFT PHASF IT $(I 3, J+1)$ IF NFCFSSARY SO THAT THF ABSOLUTF VALUE DF c. $033 \mathrm{JI}$ TS LFSS THAN 1月O.O

$n 23 J 1=\cap A T A Y(I 3, J+1)-\Gamma A T A Y(I,, J)$

IF $\{\cap 23 \mathrm{~J}] .\{\mathrm{T}, 190.0\}$

or

RT TMD $=\triangle A T Y(I 3, J+1)-3 h \cap \cdot 0$

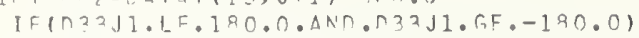

$1 D T F M D ?=7 \triangle T \triangle Y(I 3, J+1)$

$I F(\cap 23 J 1 . L T \cdot-1$. C.0)

$1 \cap T+N E 2=D \Delta T \Delta Y(I 3, J+1)+340.0$

C.5

COMTTNIIE

$I C N=I C N+1$

G 1 CINTINIE

ARMIINA THFTA, DHI.

GL TD 90

OC CONTINUE Gr Tि 100 GT TO 100

LCO CCNTINIIF

$\cap 43 \mathrm{~J} I=2 \Delta \mathrm{A} \Delta Y(\mathrm{I} 4, \mathrm{~J}+1)-\mathrm{CAT} \Delta Y(\mathrm{I} 3, \mathrm{~J})$

IF $(042 \mathrm{~J}) \cdot \mathrm{GT}, 1 \mathrm{A0.0)}$

I0 TFMP $3=$ D $\triangle T A Y(I 4, \mathrm{~J}+1)-360.0$

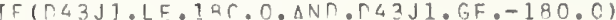

NTFMP2 $=$ DATAY $([4,1+1)$

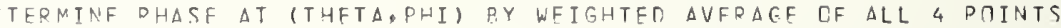

$D \Delta T A X(I D 2+1, I C)=(D F I * C T F M P I+(1.0-D F I)$ \#ATAY $(I 3, J)) *(1.0 \rightarrow D F J)$

$1+(D F I * n T F M P Z+(1.0-D F I) * D T F M P 2) * D F J$

IF $(F K Y, L T .0 .0) \Gamma \Delta T \Delta X(I R ?+I, I C)=D \Delta T A X(I R Z+1, I C)-1$ AO. 0

ATAX(IQ?+I, IC $)=A M \cap D(C \Delta T \Delta X(I P 2+I, I C)+360 . C)$

IF ( I $\Delta T \Delta \times(I R 2+1, I C), L T .0 .0) \quad D \Delta T \Delta \times(T P ?+I, I C)=0 \Delta T \Delta X(I R 2+1, I C)+360.0$

Rก तAT $\triangle \times([0), I C)=0$.

$C A T A X(I R 2+I, I C)=C$.

IF $(T C O N)-$ ICN $.1 \mathrm{~F}, \mathrm{C}) \mathrm{GO} T \mathrm{TC} 102$

IF (IDY - IQN LF. C) GO TO 101

DATAX(IRX - IRN,IC) = OATAX(IPZ,ICZOZ - ICN $=$

$10 A T A X(I R X-I R N, I C 2 D 2-I C N)=D A T A X(I R 2, I C)$

DATAX(IRX - IRN + I,IC) $=$ DATAX(IR2+1,IC2D2-ICN) =

DATAX(IRY - IRN + I,IC2D? - ICN $=0 \triangle T A X(I R 2+I+I C)$

$1 C I D A T A X(I R ?$, ICZD? - ICN) = DATAX(IRZ, IC,

$\cap A T \Delta X(I R ?+1, I C 2 D 2-I C N)=D \Delta T \Delta X(I R 2+1, I C)$

102 IF (IQY - IRN LE, O) ER TO 100

CATAX(TQY - IPN,IC) = DATAX (IR?,TC)

$D A T \Delta X(I R X-I R N+I, I C)=D \Delta T \Delta X(I R 2+1, I C)$

C WRITF FAR-FIFLD DUT INN IINIT LUOUT

CALI FFחIT (DATAX, IR2X2, ICZTחN, LUחUT)

CALCULATE MEAR-FIFLD

CALL NFKXY( ח $\triangle T \Delta X$, IR $2 \times 2$, I $\triangle 2 T O N)$

JRO? FTRMAT (IF, (TF, 1DFI2.4))

2001 FRPMAT (1HO)

PFTLION!

FND 


\section{A.1.4 SUBROUTINE NFKXY(DATA, IR2X2, IC2TON)}

PURPOSE:

To calculate an array of near-field electric field values for an antenna given an array expressing the far-field radiation pattern of the antenna.

\section{ARGUMENTS:}

DATA - A two-dimensional array which on entry contains one component of the far-field radiation pattern of an antenna expressed in polar form as a function of equaliy spaced $k_{x}$ and $k_{y}$ coordinates. The amplitudes and phases are stored in adjacent locations in memory. On exit, this array contains the near-field pattern in polar form as a function $x$ and $y$.

IR2X2 is twice the number of rows ( $k_{y}$ or $y$ values) in the array DATA.

IC2TON is the number of columns in the array DATA.

METHODS:

The expression evaluated in this subroutine is basically eq (45) repeated below.

$$
E_{y}(\underline{r})=\frac{1}{2 \pi} \sqrt{\frac{P_{0} G(0)}{2 \pi Y{ }_{0} k^{2}}} \int s_{10}(\underline{k}) e^{i|\gamma| z} e^{i \underline{K} \cdot \underline{R}} d \underline{k} .
$$

The quantity $\mathrm{s}_{10}(\underline{K})$ is the normalized transmitting coefficient and is given in terms of the far field by

$$
s_{10}(\underline{K})=\frac{\underline{E}(\theta, \theta)}{\gamma E_{y}(0)}
$$

This quantity is multiplied by the power normalization factor in front of the integral and stored in the input array on entry to the program. The integral is converted into a discrete Fourier transform (DFT) and evaluated using the FFT algorithm. The resulting summation is,

$E_{y}(\underline{r})=\frac{1}{2 \pi} \sqrt{\frac{P_{0} G(0)}{2 Y_{0} k^{2}}} \frac{\Delta K_{x} \Delta k_{y}}{E_{y}(0)} \sum_{i=-N}^{N} \sum_{j=-M}^{M} \frac{E(i, j)}{\gamma_{i j}} e^{i \gamma_{i j} z^{i K_{i j}}} e^{R_{i j}}$ 
The subroutine ETOIGAM is called for each column in order to multiply the input data by

$$
e^{i \gamma_{i y} z}
$$

This array DATA is then converted from polar to rectangular form using subroutine ARAYPTR. The Fourier transform is then performed using FFT routine FOURT and the results converted to polar form using subroutine ARAYRTP.

The results of the FFT must be corrected in two ways because of the nature of the FFT algorithm and the indexing system used in FORTRAN. First, the summation indices must be changed to 1 to $2 N(M)+1$, rather than $-N$ to $N$, as in eq (1). Second, the output is in a range 0 to $2 \pi$ rather than $-\pi$ to $\pi$. The first is equivalent to a shift in origin, and, by the shifting theorem of Fourier analysis, produces a linear phase shift after transformation to the near field. The second effect causes the center of the near field to be located at the point $(1,1)$ in the output array and the negative and $x$ - and $y$-positions are in the outer portions of the array. The output data are rearranged in order to place the center of the near field at the center of the array. This is accomplished using subroutine SWAP. The phase shift is corrected in PHSCOR2.

The data in corrected form now reside in array DATA. Printer plots are produced using subroutine PLT120R. This subroutine uses library functions, ATAN and subroutines ARAYPTR, ETOIGAM, FOURT, SWAP, and PHSCOR2.

\section{SYMBOL DICTIONARY:}

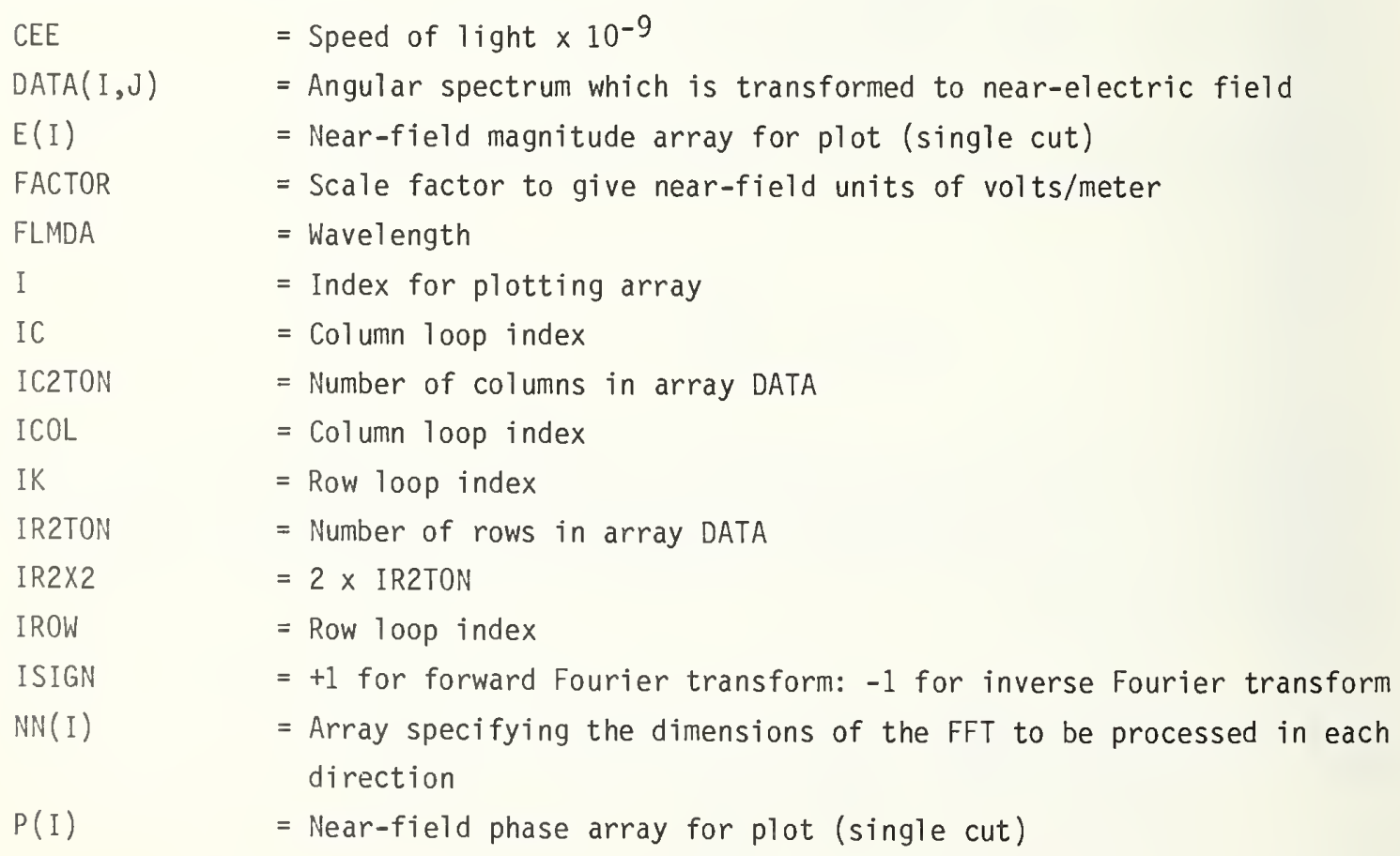


PI

PIX2

RTD

$X(I)$

XMAX

XMIN $=\pi=3.14159 \ldots$.

$=2 \pi$

= Radian to degree conversion factor $=180 / \pi$

$=x$-coordinate array used in near-field plots

= Maximum value of $x$ for plots

= Minimum value of $x$ for plots 
CALL DLTI?OR(X, E, XMAX, XMIN, 10., 0., IC 2TON, IH*, 1, 1) PRINT ?OO3, C.SSEIN, 1OHY-7. RLANF, 1OHAMDLITUDF

C $\triangle L L$ PLTIPOR (Y, E, YMAX, YMIN, 10., O., IC?TON, 1H*, 1, I) ODINT 2003 . CASFID, 1 CHX-Z OLANF, BOHAMRLITLDE

CALL DLTIZOR(Y, D, YMAX, YMIN, 360., 0., IC2TON, 1H*, 1, 1) PRTNT ?OO3, CASFIO, 10HX-? PLANF, IOHPHASF 
QETIIRN

NFKXY

PחC, - CONAT1/1/,TE4, \#CENTERLINE DATA*, /1, T37, *X-Z PLANE*, T97, \#Y-Z PLANFKXY IVF*, 1, T22, *X*,T40,*AMD*,T50,*PHASE*,T82,*Y*,T100, AMP*, T119, *PHASENFKXY ?*)

NFKXY

NFKXY

NFKXY

DNO? FCOMATITA, GF 20.4

20 C F FPMAT $(1.5 x, 9 \Delta 10,5 x, 2 \Delta 10)$

NFKXY

80

FNn 


\section{A.1.5 SUBROUTINE \\ ETOIGAM(DATA ( 1 , ICOL) , NROW, NCOL, ICOL, ISGN, FLMDA, DELX,DELY,DIST)}

\section{PURPOSE:}

To multiply each element of complex array DATA by the factor exp(+ird).

\section{ARGUMENTS:}

DATA is a two-dimensional complex array in polar form whose magnitude and phase are adjacent in storage.

NROW is the number of rows in array DATA.

NCOL is the number of columns in array DATA.

ICOL is column number of the data to be operated on.

ISGN $= \pm 1$ depending on whether DATA is to be multiplied by $\exp ( \pm i \gamma d)$.

FLMDA operating wavelength.

DELX $x$-increment of desired near-field data.

DELY $y$-increment of desired near-field data.

DIST spacing between antenna reference point and desired near-field plane.

\section{METHODS:}

The subroutine does not employ complex arithmetic. It is assumed that the numbers in array DATA are the magnitude and phase stored in adjacent locations. If DATA contains complex data in real and imaginary form, a call to ARAYRTP must be made prior to the call to ETOIGAM. The pertinent relationships are

$$
\begin{aligned}
\text { DATA } & =\text { DATA } e^{i \gamma d} \\
\gamma & =\sqrt{k^{2}-k_{x}^{2}-k_{y}^{2}} \\
k & =\omega \sqrt{\mu \varepsilon} \\
k_{x} & =k \sin \theta \cos \emptyset \\
k_{y} & =k \sin \theta \sin \emptyset .
\end{aligned}
$$

Because DATA is assumed to be in magnitude, argument form, we calculate

$$
\operatorname{ARG}(D A T A)=A R G(D A T A)+\gamma d
$$

for $\gamma$ real, and

$$
\operatorname{MAG}(D A T A)=\operatorname{MAG}(D A T A) \operatorname{Exp}(-\gamma d)
$$

for $\gamma$ imaginary

$\gamma$ is computed from the row and column positions of the data elements. 


$$
\begin{aligned}
& k_{y}=\frac{2 \pi(\text { IROW }-N R O W / 2)}{N R O W \cdot \Delta_{y}} \\
& k_{x}=\frac{2 \pi(\text { ICOL }-N C O L / 2)}{N C O L \cdot \Delta_{x}}
\end{aligned}
$$

Array DATA is assumed to correspond to points equally spaced in $k_{x}, k_{y}$ with $k_{x}=k_{y}=0$ being the center point of the array.

SYMBOL DICTIONARY:

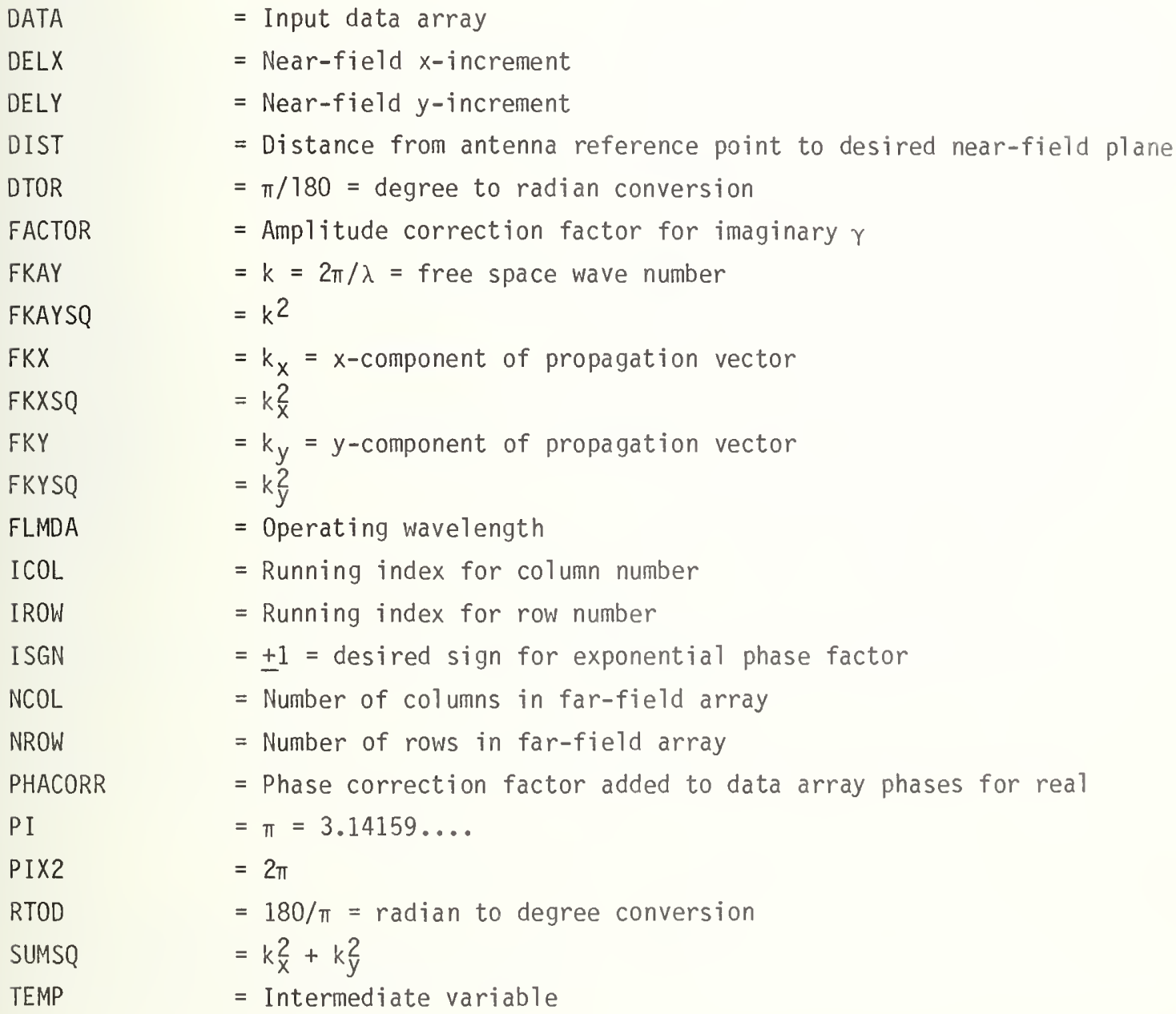


RTก = $100 . / \mathrm{PI}$

$\cap T \cap R=1 .$, NTIO

$F K X=P I X 2$ (ICกL - (NFOL / 2) , DELX / NCOL

$\mathrm{FK} \times S ?=F K X \neq \$$

FTCIGAM

ETOIGAM

ETOIGAM

ETOIGAM

ETOIGAM

ETDIGAM

ETOI GAM

ETOI GAM

ETOI GAM

ETOIGAM

ETOIGAM

FTOI GAM

IF (NIRTW.LT. 1) GO TO 13J

OR $1>0$ IRTW = I, NRTW

FKY $=$ PIX? \# (IRIW-(NRDW/2)) / DELY, NROW

ETOIGAM

ETOI GAM

FKYSO = FKY *

ETOIGAM

SIIMSO $=F K X S O+F K Y S G$

?

PHACADR $=0.0$

F $\triangle C T C^{Q}=1.0$

c

IF (SIIMSO. GT. FKAYSC) GO TO $10 C$

25

OHACDRR = ISGN \# CORT (FKAYSO - SUMSO) \# DIST \# RTOD

ETOIGAM

FTUIGAM

ETOI GAM

ETO I GAM

ETOIGAM

ETOIGAM

ETOIGAM

ETOI GAM

ETOIGAM

FT CIGAM

EIUIGAM

ETOIGAM

ETO I GAM

ETRI GAM

CTIIGAM

ETOIGAM

ETOIGAM

ETOIGAM

ETOIGAM

ETOIGAM

ETOI GAM

ETOIGAM

ETO IGAM

ETOIGAM

ETOIGAM

ET OI GAM

1500 FחDMAT (1X, 4I10, 5F12.3, /1)

1510 FIRMAT (1X, ?T5. 8F1?.3)

RFTUPN

ETOIGAM

$$
\text { END }
$$




\section{A.1.6 SUBROUTINE PHSCOR2(DATA, NRX2, NCOL)}

PURPOSE:

To correct the phase of the near-field data which arises because the reference point of the FFT algorithm is the point $(1,1)$ rather than the center of the far-field array.

ARGUMENTS:

DATA is a two-dimensional array containing the complex near-field data in polar form. Amplitude and phase in degrees are located adjacent in storage.

NRX2 is twice the number of rows in the array DATA.

NCOL is the number of columns.

METHODS:

As shown by the shifting theorem, a shift in coordinates in one domain introduces a linear phase shift in the transformed domain. This subroutine corrects for the phase shift which occurs as a result of the different reference points of far-field pattern and the FFT algorithm. The shift added because of this change of origin is

$$
e^{i(a x+b y)}
$$

where $a$ and $b$ are the shifts in far-field origin in the $k_{x}$ and $k_{y}$ directions respectively and $x$ and $y$ are the coordinates of the specific near-field point.

The subroutine adds a phase shift equal to

$$
-180^{\circ}\left[\left(\frac{\mathrm{NCOL}-2}{\mathrm{NCOL}}\right)(\mathrm{ICOL}-1)+\left(\frac{\mathrm{NROW}-2}{\mathrm{NROW}}\right)(\mathrm{IROW}-1)\right]
$$

to the phase of each complex number in the array in order to compensate for the above shift. It is assumed in this factor that the center of the far-field pattern lies at (NROW/2,NCOL/2).

An additional phase of $90^{\circ}$ is added to each element in order to allow the near-field phase to be conveniently plotted in the range $0^{\circ}-360^{\circ}$.

This subroutine uses intine functions FLOAT and INT. 
SYMBOL DICTIONARY:

= Phase correction for column ICOL

C2

= Phase correction for row IROW

CONST1

= Column phase increment

CONST2

= Row phase increment

DATA

= Input data array

ICOL

= Column loop index

I02

$=\mathrm{IROW} / 2$

IROW

= Row loop index

NCOL

= Number of columns in array DATA

NRX2

= Twice the number of rows in array DATA

TEMP

= Intermediate variable, the corrected phase at point (I02, ICOL) 
1

$2 n$

$? 5$
SURRTUTTNF PHSCOR2(OATA, NRX?. NCDL)

CIMFNSITN DATACNRX2, NCRL)

NROW = NRX? / ?

C

CINST1 = $190 . * F L M A T(N C M L-2) / F L D A T$ (NCOL) CחNST? = -18O.*FLחAT(NRחW - ?)/FLOAT(NROW) IF (NCRL \&T. I) GO Tח 130

DC $100 \mathrm{TCOL}=1$, NCCL

$C_{1}=$ CMNST1 $\#(I C O L-1)$

IF (NRX? $L T \cdot 2)$ GO TO 110

Dก 100 IROW = ?, NRX?, ?

$I \cap 2=[R \cap W /$,

$\mathrm{C} 2=$ CINST? * (IT? - 1$)+\mathrm{C} 1$

TEMP $=$ DATA (IROW, ICQL $+C 2+90$.

TEMP = TFMR - INT(TFMD, 360.)*360.

IF (TEMP $:$ T $: 0.1$ TEMP $=$ TEMP +360.

DATA(IDחW, TCOL) $x$ TFND

100 CONTINUF

110 CINTINIJF

130

CINTINUIF

c

RFTIIRN

FND
PHSC OR 2

PHSCOR2

PHSCOR 2

PHSCOR 2

RHSCOR 2

PHSCOR?

PHSC QR 2

PHSCDR?

PHSCOR 2

PHSCOR2

PHSCOR2

PHSCOR 2

PHSCOR 2

PHSCOR?

RHSCOR?

PHSCOR 2

PHSCOR 2

PHSCOR 2

PHSCOR 2

PHSCRR?

PHSCOR ?

PHSCOPZ

PHSCOR2

PHSCOR2

PHSCOR2

PHSCOR 2
1
2
3
4
5
6
7
8
9
0
1
2
3
4
5
6
7
8
9
20
21
22
23
24
25
26 
PURPOSE:

To perform the rearrangement of data necessary to place center of near field at center of near-field data array.

\section{ARGUMENTS:}

NRX2 is twice the number of rows in the array DATA.

NCOL is the number of columns in the array DATA.

DATA is an array containing the near-field pattern of an antenna which is to be rearranged.

\section{METHODS:}

The FFT algorithm fundamentally takes data over a range of $0-2 \pi$ and transforms them into a domain of $0-2 \pi$. Suitable scaling is employed to fit the far-field (angular spectrum) and near field ( $x-y$ position) into these ranges. The negative portion of the $x-y$ range occurs from $\pi$ to $2 \pi$. Thus, to have a continuous near field at $x, y=0$, the data are rearranged.

The rearrangement is done in $\mathrm{place}$, the rearranged array replacing the original one in core, requiring only three temporary storage locations. The rearrangement takes place in two steps. First, the edges of the array are moved to the center and the center to the edges by columns. The process is then repeated by rows.

The array DATA contains complex numbers which may be in either polar or rectangular form. This routine does not use complex arithmetic.

\section{SYMBOL DICTIONARY:}

$\begin{array}{ll}\text { DATA } & =\text { Complex array to be rearranged } \\ \text { ICOL } & =\text { Column loop index } \\ \text { ICPNC } & =\text { Intermediate subscript } \\ \text { IROW } & =\text { Row loop index } \\ \text { IRPNR } & =\text { Intermediate subscript } \\ \text { NCM1 } & =\text { NCOL }-1 \\ \text { NCOL } & =\text { Number of columns in DATA } \\ \text { NCO2 } & =\text { NCOL } / 2 \\ \text { NROW } & =\text { Number of rows of compTex data } \\ \text { NRX2 } & =2 \cdot \text { NROW }=\text { dimension of DATA in row directio. } \\ \text { NR2M2 } & =\text { NRX2 }-2\end{array}$


= Intermediate variable

TEMP 1

= Intermediate variable

TEMP2

= Intermediate variable 
S-MIVTNG FIEFS IF ARRAY TR CENTER AND VICE VERSA RY COLUMNS

IF (NRY?. LT, 1$)$ GO TO 220

ก 200 TROH = 1 , NRX?

IF INCM? . LT. I) GM TR 210

In $2 \cap 0$ TCOL $=1$, NCח 2

$I C P N C=I C \cap L+N C O Z$

$T F M P=D A T A(I R \cap W, I C P N C)$

OATA (IR OW, ICPNC) = DATA (IROW, JCOL)

$2 \cap 0$ ПATA(IR ПW. ICCL) = TFMP

5] $O$ CINTINIIF

?DO CONTTNIIF 


\section{A.1.8 SUBROUTINE ARAYPTR(DATA, NRX2, NCOL)}

\section{PURPOSE:}

To convert a two-dimensional complex array from polar form to rectangular form or from rectangular form to polar form (ENTRY ARAYRTP).

\section{ARGUMENTS:}

DATA is a two-dimensional complex array whose real and imaginary parts are adjacent in storage, such as FORTRAN IV places them. On exit, DATA contains adjacent amplitudes and phases.

NRX2 is twice the number of rows in DATA.

NCOL is the number of columns in DATA.

\section{ENTRY POINT:}

ARAYRTP performs rectangular to polar conversion.

\section{METHODS:}

This subroutine does not use complex arithemtic. However, array DATA is stored in the same fashion as is required by FORTRAN IV for complex numbers. Thus, while the subroutine operates on a complex array, complex FORTRAN functions are not used.

\section{ARAYPTR}

DATA(IROW,ICOL) contains magnitude of complex number.

DATA(IROW+1, ICOL) contains phase of complex number, expressed in degrees.

$\operatorname{Re}(D A T A)=\mid$ DATA $\mid \cos ($ ANGLE $(D A T A))$.

$\operatorname{Im}($ DATA $)=\mid$ DATA $\mid \sin (\operatorname{ANGLE}($ DATA $))$.

2. ARAYRTP

DATA(IROW, ICOL) contains real part of the complex number.

DATA(IROW+1, ICOL) contains imaginary part of the complex number.

$$
\begin{aligned}
& \mid \text { DATA } \mid=\sqrt{[\operatorname{Re}(\text { DATA })]^{2}+[\operatorname{Im}(\text { DATA })]^{2}} . \\
& \text { ARG }(\text { DATA })=\tan ^{-1}\left[\frac{\operatorname{Im}(\text { DATA })}{\operatorname{Re}(\text { DATA })}\right] \times 180^{\circ} / \pi .
\end{aligned}
$$

This subroutine uses library functions, SIN, COS, ATAN2, and SQRT. 
SYMBOL DICTIONARY

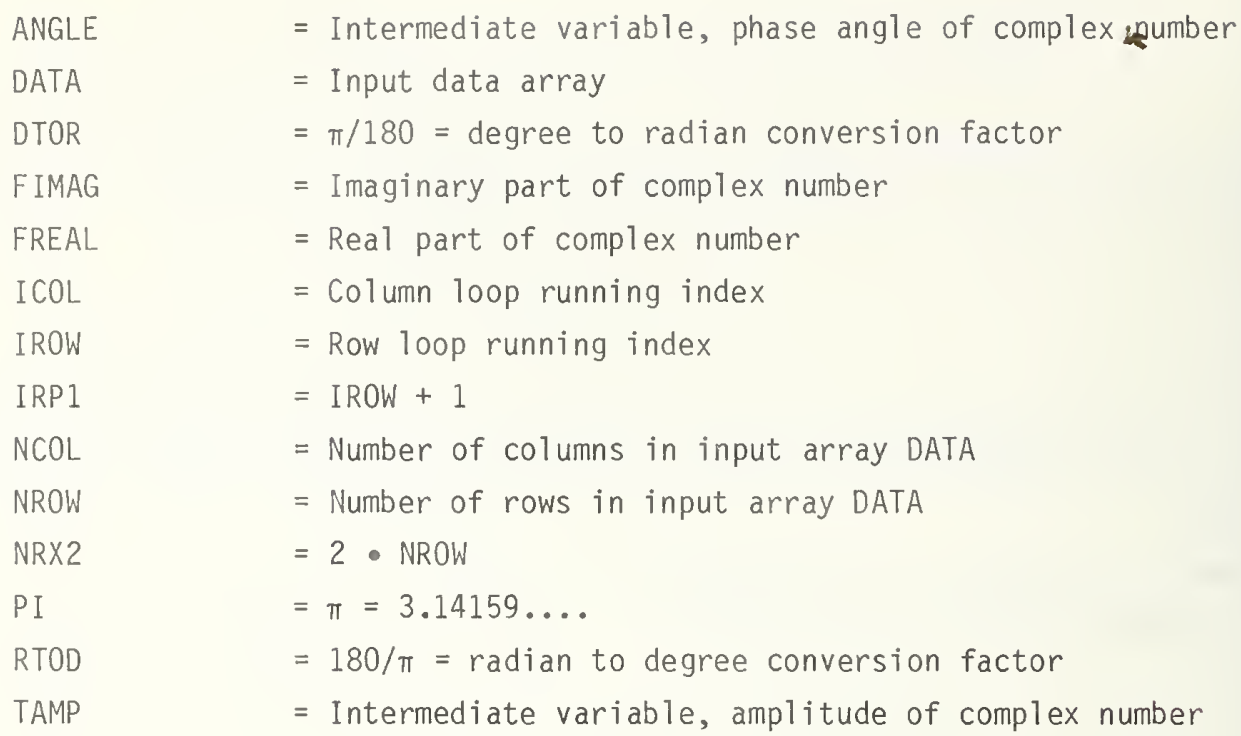


5

$P I=3.1415026536$

DTOD = 1 RO, PI

ПTחD $=1 ., D T \cap \Gamma$

$1 ?$

15

2า

100

117

$1 ? n$

$1 ?$

$r$

TF (venl. LT, I) G T T 130

I) IPC IOAL $=1$, NCOL

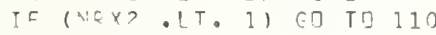

$\Pi \cap 1 \cap 0$ I $\cap N=1, V P \times 2,2$

$I P P I=I P \cap \mathrm{W}+1$

$T \angle N D=M A T A(I P \cap W, I C[L)$

$A N C L F=2 A T A(I R D I, I C C L) \neq N T \cap R$

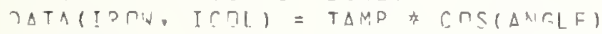

DATA (IRDI, TCML) = TAMP $\#$ SIN( $\triangle N G L E)$

CONTINUF

COMT TNIIE

Cmitinith

CLNTTUIIF

DFTIIPN

25

ENTRY $\triangle P \triangle Y R T R$

$D I=2.1415925536$

गTH = IPT., PI

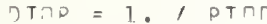

$3 n$

c

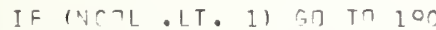

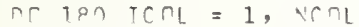

IF (Nox? :LT. I) SO TR 170

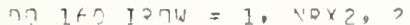

$T \& D T=T D \cap N+1$

FOFAL = NATA(IDNW, ICOL)

ETVAS $=2 \Delta T \Delta(I P D I, T C R L)$

DATA (IORA, TCCL) = SORT(FRFAL \# FRFAL + FIMAG \# FIMAG)

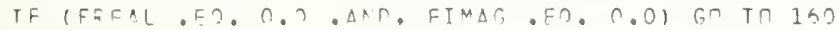

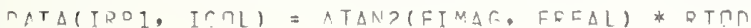

40

$1<0$

170

IRO

190

45
CANTIMUIT

CANT TN!IF

CONTTNIHF

CONTTNIIF

J $2 T+10 \mathrm{~N}$

Lan $\triangle R \Delta Y P T R$

$\triangle R A Y P T R$

$\triangle R \triangle Y P T P$

$\triangle P \triangle Y P T R$

$\triangle P \triangle Y P T R$

$\triangle D \triangle Y R T P$

$\triangle D \triangle Y P T R$

$\triangle R \triangle Y P T P$

$\triangle Q \triangle Y P T R$

AP $\triangle Y P T R$

$\triangle R \triangle Y P T R$

$\triangle R \triangle Y P T R$

APAYRTR

$\triangle Q \triangle Y P T R$

$\triangle R A Y P T P$

$\triangle R \triangle Y P T R$

$\triangle R \triangle Y P T R$

$\triangle Q A Y P T R$

$\triangle R \triangle Y P T R$

$\triangle D \triangle Y R T P$

$\triangle D \triangle Y R T R$

$\triangle R \triangle Y P T R$

$\triangle R \triangle Y P T R$

$\triangle R A Y P T R$

$\triangle D \triangle Y P T R$

$\triangle D A Y R T R$

$\triangle R \triangle Y P T R$

$\triangle D \triangle Y P T P$

$\triangle R A Y P T R$

$\triangle R \triangle Y R T R$

$\triangle O \triangle Y R T O$

$\triangle P \triangle Y D T P$

$\triangle P A Y R T R$

$\triangle R \triangle Y P T P$

$\triangle R \triangle Y R T R$

$\triangle R A Y R T R$

$\triangle R A Y R T D$

$\triangle R A Y R T R$

$\triangle R \triangle Y P T R$

$\triangle R \triangle Y R T R$

$\triangle R A Y P T R$

$\triangle Q \triangle Y Q T R$

ARAYPTR

$\triangle R A Y P T R$

ADAYPTD 
PURPOSE:

This subroutine writes the array DATA out to logical unit LUOUT. A header record is written as the first record of the file.

\section{ARGUMENTS:}

DATA is the array to be written out.

NRX2 is the number of floating point numbers in a row.

NCOL is the number of columns.

LUOUT is the logical unit on which file is to be written.

METHODS:

A file consisting of $\mathrm{NCOL}+1$ records is written on unit LUOUT. The first record is an identification (ID) record, and each of the NCOL rows in the array DATA is a record. The records are written using unformatted WRITE statements.

The ID record consists of ten ten-character words, these are listed below

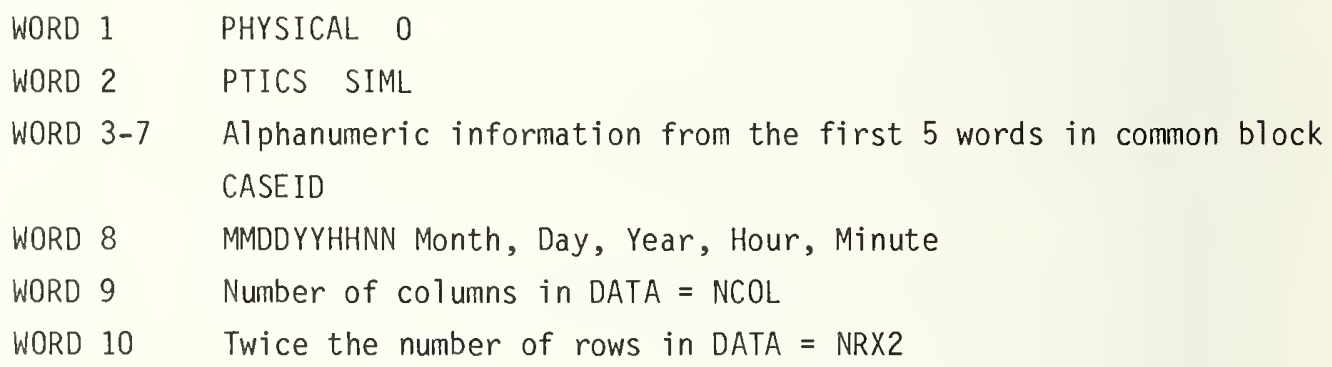

Words 1 through 8 are written in Hollerith format, and words 9 and 10 are written in integer (I) format. The ID record may be read with a (8A10,2I10) format. The date and time for word 8 are generated by calls to DATE and TIME and are thus the date and time when the output file was created.

The subroutine uses library functions DATE and TIME.

SYMBOL DICTIONARY:

$\begin{array}{ll}\text { CASEID } & =\text { Hollerith identification supplied from calling program } \\ \text { DATA } & =\text { Array to be written to unit LUOUT } \\ \text { DT } & =\text { Date information obtained from function DATE } \\ \text { DY } & =\text { Day of month }\end{array}$




$\begin{array}{ll}\text { HR } & =\text { Hour } \\ \text { I } & =\text { D0 loop index } \\ \text { IC } & =\text { Column loop index } \\ \text { ID } & =\text { Identification array } \\ \text { IR } & =\text { Row loop index } \\ \text { LUOUT } & =\text { Output logical unit number } \\ \text { MN } & =\text { Minute } \\ \text { MON } & =\text { Month } \\ \text { NCOL } & =\text { Number of columns in DATA } \\ \text { NRX2 } & =\text { Number of rows in DATA } \\ \text { SC } & =\text { Second } \\ \text { TM } & =\text { Time information obtained from function TIME } \\ \text { YR } & =\text { Year }\end{array}$


FND

FOU

FFOUT

FFOUT

FFOUT

FFOUT

FFOUT

FFOUT

FFOUT

FF CUT

FF CUT

FFOUT

FFOUT

FFOUT

FFOUT

FFOUT

FFOUT

FFOUT

FFOUT

FFCUT

FFOUT

FFOUT

FFOUT

FFOUT

FFCUT

FFOUT

FFOUT 


\section{A.1.10 SUBROUTINE FOURT(DATA, NN, NDIM, ISIGN, IFORM, WORK)}

PURPOSE:

To compute the discrete Fourier transform of the array DATA using the fast Fourier transform algorithm.

\section{ARGUMENTS:}

DATA is a multidimensional complex array whose real and imaginary parts are adjacent in storage, such as FORTRAN IV places them.

NN is an array giving the lengths of the array in each dimension.

NDIM is the number of dimensions of the array DATA, hence the number of elements in array NN.

ISIGN is +1 for a forward transform -1 for a reverse transform.

IFORM If al1 imaginary parts of the input array are zero (input array is real), set IFORM $=0$ to reduce running time by approximately 40 percent, otherwise set IFORM $=+1$.

WORK if all dimensions of DATA are not integral powers of 2, specify array WORK in calling routine with dimension greater than largest non $2^{k}$ dimension, otherwise set WORK $=0$.

METHODS:

Using the Fast Fourier transform algorithm, FOURT replaces the array DATA with its discrete Fourier transform given by

$$
\operatorname{TRANSFORM}(K 1, K 2, \ldots)=
$$

$\sum^{N N(1)} \sum^{N N(2)} \operatorname{DATA}(J 1, J 2) e^{i 2 \pi I S I G N}\left\{\frac{(J 1-1)(K 1-1)}{N N(1)}+\frac{(J 2-1)(K 2-1)}{N N(2)}+\ldots\right\}$

$$
\sum_{\mathrm{J} 1=1} \quad \sum_{\mathrm{J} 2=1}
$$

For a more complete description of the subroutine and its usage, see the comments included at the beginning of its listing or the supplementary comments by the programmer, Norman Brenner of MIT.

Uses external library functions COS, SIN, FLOAT, and MAXO.

Note: Brenner, Norman, "FOUR2 and FOURT program description," private communication, 1968. 


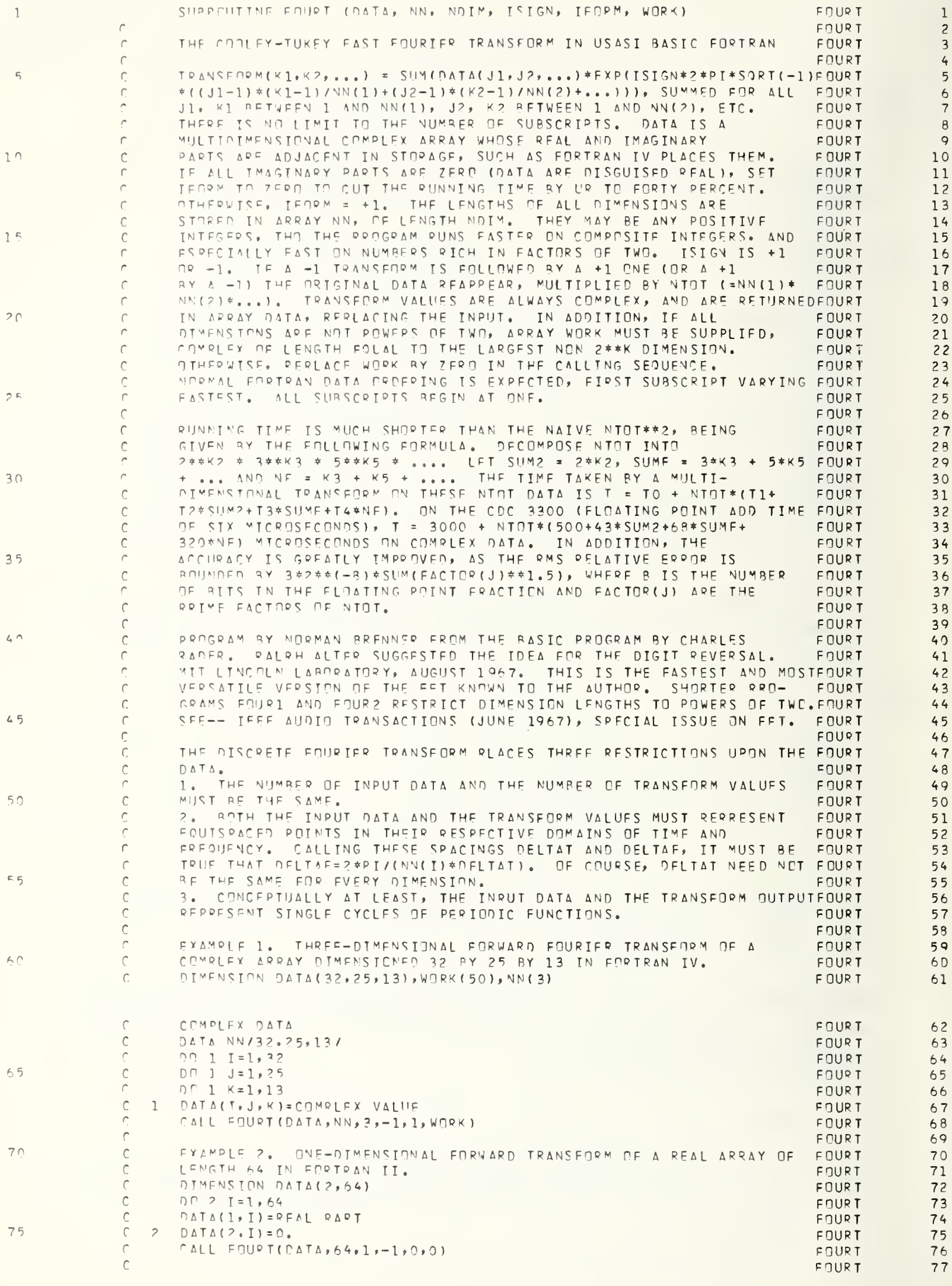


FOURT $\quad 78$

FIURT

FOURT 80

FOURT $\quad 81$

FIURT 82

FOURT 83

FDURT 84

FOURT 85

FDURT 86

FDURT 87

FIURT AB

FOURT 89

FRURT 90

FIURT 91

FTURT 92

FIURT 93

FIURT 94

FOURT 95

FIURT 96

FDURT 97

FOURT 98

FIURT 99

FOURT 100

FOURT 101

FOURT 102

FOURT 103

FOURT 104

FOURT 105

FOURT 106

FIURT 107

FOURT 108

FOURT 109

FOURT 110

FOURT 111

FIURT 11?

FIURT 113

FIURT 114

FOURT 115

FIURT 116

FOURT 117

FOURT 119

FIURT 119

FOURT 120

FOURT 121

FOURT 122

FIUURT 123

FIURT 124

FIURT 125

FEURT 126

FOURT 127

FIURT 128

FRURT 120

FDURT 130

FOURT 131

FOURT 132

FOURT 133

FOURT 134

FIURT 135

FTURT 135

137 


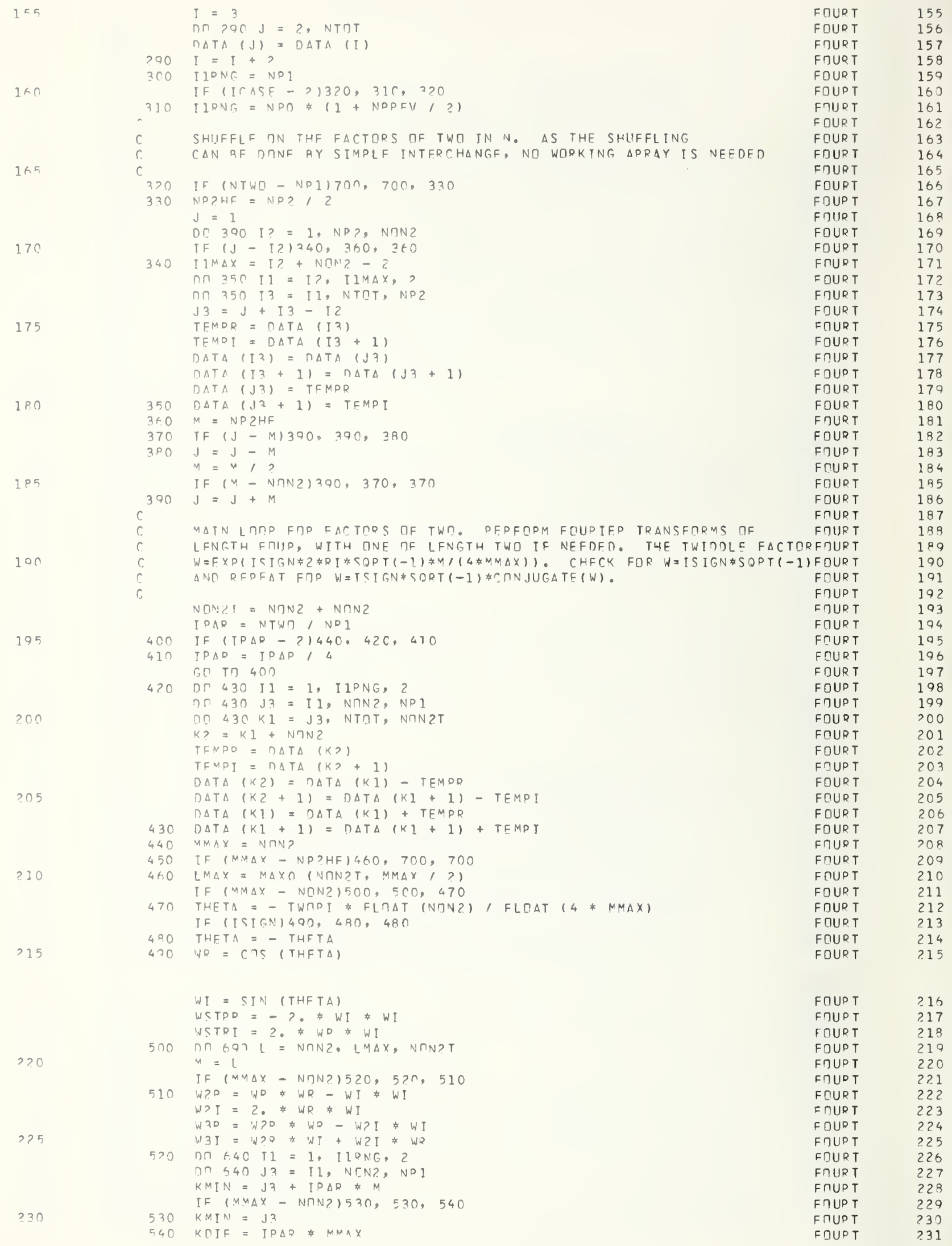


ASO TFMDE = WR

$690 W I=W I * W S T D R+T E M P R$ WSTPI + WI FOURT

C MAIN LIMP FIR FACTORS NTT EOUAL TO TWO. ADRLY THE TWIDOLE FACTOR $N=F \times P(I S I S N * 2 * P I * S Q Q T(-1) *(J 2-1) *(J 1-J 2) /(N P 2 * I F R I))$. THEN 


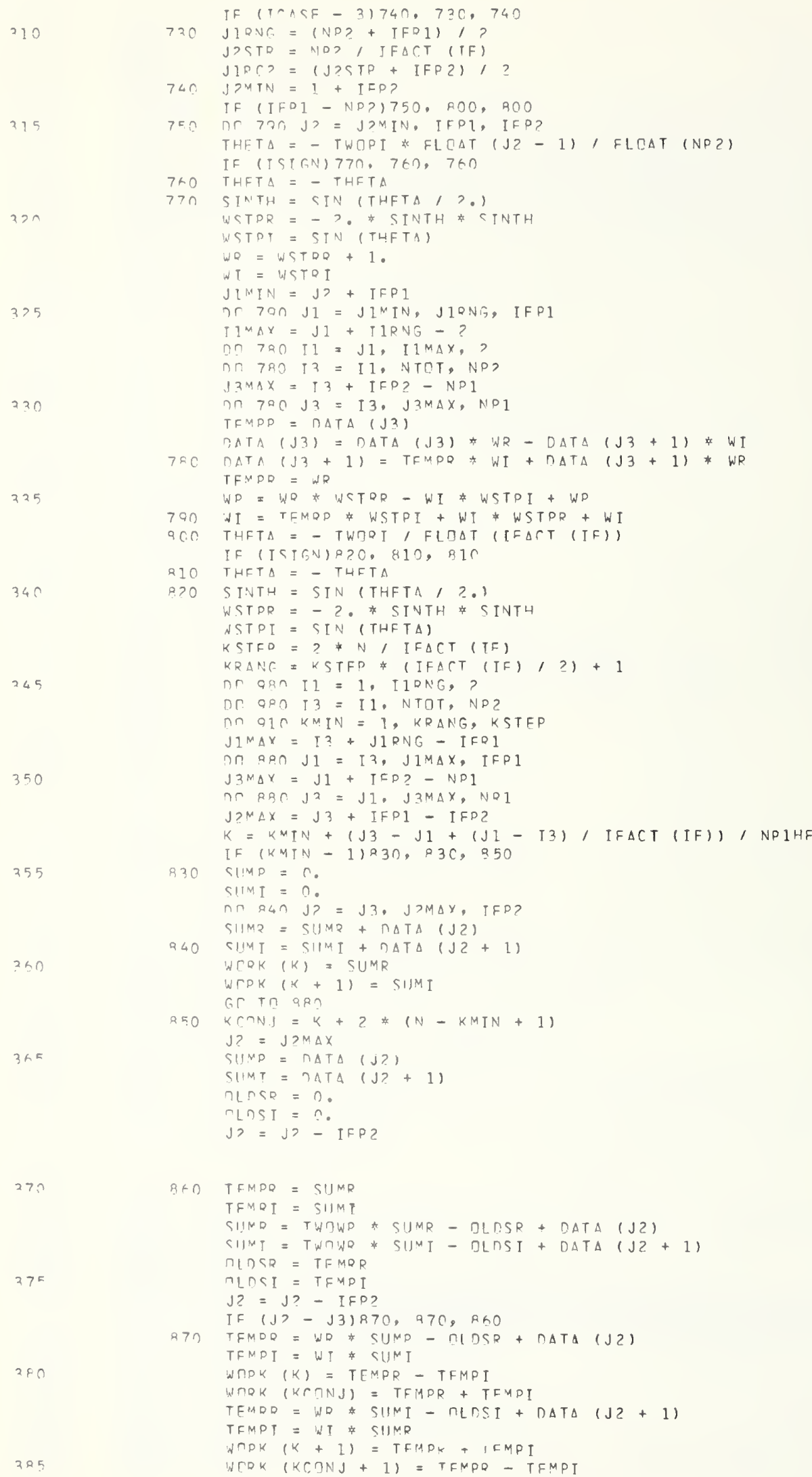


200

395

400

405

410

415

420

$4>5$

420

425

440

445

1030

450

455

$4 \in 0$
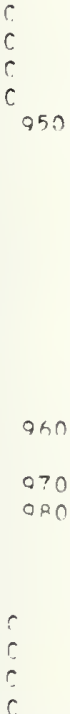

1000
RDO CONTINIIL

IF IKMTN - I)ROO, BQC, 900

ROO

$R D+1$

$W I=W C T R I$

G T T Q

$O C O$ TFMPR = WD

$W D=W R$ WSTDR - WI *WSTRI + WD

$W I=T F M D R * W S T R I+W I * W S T R R+W I$

$010 T W \cap W R=W R+W R$

IF (IA $15 E-3) 030,92 \mathrm{C}, 930$

020 IF (TFRI- NR2) $950,920,930$

$930 K=1$

$I ? M \Delta x=I 3+M P>-N D I$

Dח 940 I? = I3, I2MAX, NRI

DATA (I?) = WTRK (K)

$D T A(I 2+1)=W \cap R K(K+1)$

$040 k=k+?$

Gก Tก จRO

950

CCMPLFTE $\triangle$ RFAL TRANSFORM IN THE IST DIMENSIDN, N DDD, BY CONJUIGATF SYMMFTRIES AT EACH STAGF。

$J 3 M \Delta X=I^{2}+I F P Z-N D I$

$\cap \cap 97 \cap J 3=I 3, J 3 M A X, N P I$

$J 2 M A X=J 3+N R>-J P S T P$

ก0 $070 \mathrm{~J} 2=\mathrm{J}, \mathrm{J}, \mathrm{J} 2 \mathrm{M} \Delta \mathrm{X}, \mathrm{J}, \mathrm{JT} \mathrm{T}$

$J M A X=J ?+J 1 P G ?-I F D ?$

$J I C N J=J 3+J P M A X+J \geqslant S T R-J 2$

$\cap 0070 \mathrm{~J} 1=\mathrm{J} 2, \mathrm{~J} 1^{M} \Delta \mathrm{X}$, IFP?

$K=1+J 1-I^{2}$

DATA $(J 1)=W \cap R K(K)$

$\triangle A \cap(J)+1)=H \cap P K(K+1)$

IF (J1-J2) 1970,070, ahO

QRO DATA $\{J I C N J)=$ WORK (K)

ก ATA $(J T C N J+1)=-$ WחOK $(K+1)$

$0 ? 0 J 1 C N^{\prime} J=J 1 C N J-I F D 2$

ORO T CNTTNUF

$I F=I F+1$

$[F R]=[F P$ ?

IF (IFQ1 - NDI)Q9?, 990,700

Gon CO Tก (1260, 1180, 1260, 1000), ICASE

NHAIF $=\mathrm{N}$

$N=N+N$

THFTA $=-$ TWMPI / ELU:AT (V)

TF (ISTGN) 102ก, IOIO, 1C10

1010 THFTA $=-$ THFTA

1020 SINTH $=$ SIN (THFTA, ?.)

WSTRR $=-?$ \# SINTH * CINTH

$W S T R I=S T N(T H F T A)$

$N R=N C T D D+1$.

$N I=M S T D I$

$I M I N=3$

JMIN $=$ ? NHALF - 1

Gก T? 1050

$\mathrm{J}=\mathrm{JMTN}$

CC 1ח4n I = ININ, NTOT, NO?

SIJMD $=(\cap \Delta T \Delta$ (I) + ПATA (J)) / ?

SIINI $=(10 \Delta T(I+1)+\operatorname{CAT\Delta }(\mathrm{J}+1)) / 2$.

$\cap T F P=(O \Delta T \Delta(I)-O \Delta T A(J)) /$ ?

NIFT $=(\cap \Delta T \Delta(I+1)-\cap \Delta T \Delta(J+1)) / 2$.

TFMPR = WR * SUMI + WI * DIFP

$T F M D I=W I \leftarrow S U M I-W R \notin D I F D$

$O A T A(I)=$ SIMMR + TFNPR

$\cap A T A(I+I)=$ D IFI + TFNRI

DATA (J) = SUMR - TFMDD

$D \Delta T \Delta(J+1)=-$ DFI + TEMDI

$1 \Gamma 40 \mathrm{~J}=\mathrm{J}+\mathrm{NR}$ ?

IMIN $=I M I V+2$

$J M I N=J M I N-?$

$T F M P D=W D$

$W R=W R$ *WSTDR - WI \#WSTDI + WR

$W I=T F M P R$ *WSTPI + WI $W S T P R$ *WI

$105 \mathrm{C}$

IF (IMIN - JMIN) 1030, 1040, 1090

\begin{tabular}{|c|c|}
\hline F QUR T & 386 \\
\hline FDUDT & $3 R 7$ \\
\hline F OURT & 389 \\
\hline F OUR T & 389 \\
\hline FOUPT & 390 \\
\hline FOURT & 391 \\
\hline F CUR T & 392 \\
\hline FCURT & 303 \\
\hline FDURT & 394 \\
\hline FOUDT & 395 \\
\hline EחUR T & $39 \pi$ \\
\hline F OURT & 397 \\
\hline FIURT & 398 \\
\hline FOURT & 390 \\
\hline FOLPT & 400 \\
\hline$F \cap \cup R T$ & 401 \\
\hline FOURT & $40 ?$ \\
\hline FOURT & 403 \\
\hline$F \cap \cup R T$ & 404 \\
\hline F OUD T & 405 \\
\hline F CURT & 405 \\
\hline FOURT & 407 \\
\hline F RUP.T & 408 \\
\hline F IUR T & 409 \\
\hline FחURT & 410 \\
\hline F OUP T & 411 \\
\hline FOURT & 412 \\
\hline FOURT & 413 \\
\hline FDURT & 414 \\
\hline FTUPT & 415 \\
\hline FOURT & 416 \\
\hline FOURT & 417 \\
\hline FDURT & 413 \\
\hline FOUPT & 419 \\
\hline FRURT & 420 \\
\hline FRUPT & 421 \\
\hline FOUIRT & 422 \\
\hline FOLIRT & 423 \\
\hline FOURT & 424 \\
\hline FDURT & 425 \\
\hline F OUR T & 426 \\
\hline FחUR T & 427 \\
\hline F TUR T & $4 ? 8$ \\
\hline FOLIR T & 429 \\
\hline FOURT & 430 \\
\hline FDUPT & 431 \\
\hline F OUR T & 432 \\
\hline F CIUR T & 433 \\
\hline FIURT & 434 \\
\hline FOURT & 435 \\
\hline F QUR T & 436 \\
\hline FOURT & 437 \\
\hline FDURT & 438 \\
\hline$F \cap \cup R T$ & 430 \\
\hline$F O L I R T$ & 440 \\
\hline FOURT & 441 \\
\hline FQURT & $44 ?$ \\
\hline FOURT & 443 \\
\hline F RIJRT & 444 \\
\hline FOUR T & 445 \\
\hline FחUP T & $44 h$ \\
\hline FCURT & 447 \\
\hline FDURT & 448 \\
\hline FDUPT & 440 \\
\hline FCURT & 450 \\
\hline FCURT & 451 \\
\hline FOURT & 452 \\
\hline FOUP T & 453 \\
\hline$F \cap \| R T$ & 454 \\
\hline FחURT & 455 \\
\hline FOURT & 456 \\
\hline FDURT & 457 \\
\hline FOUR T & 458 \\
\hline FDURT & 450 \\
\hline FחUR T & 460 \\
\hline FחURT & 461 \\
\hline FOUR T & 462 \\
\hline
\end{tabular}




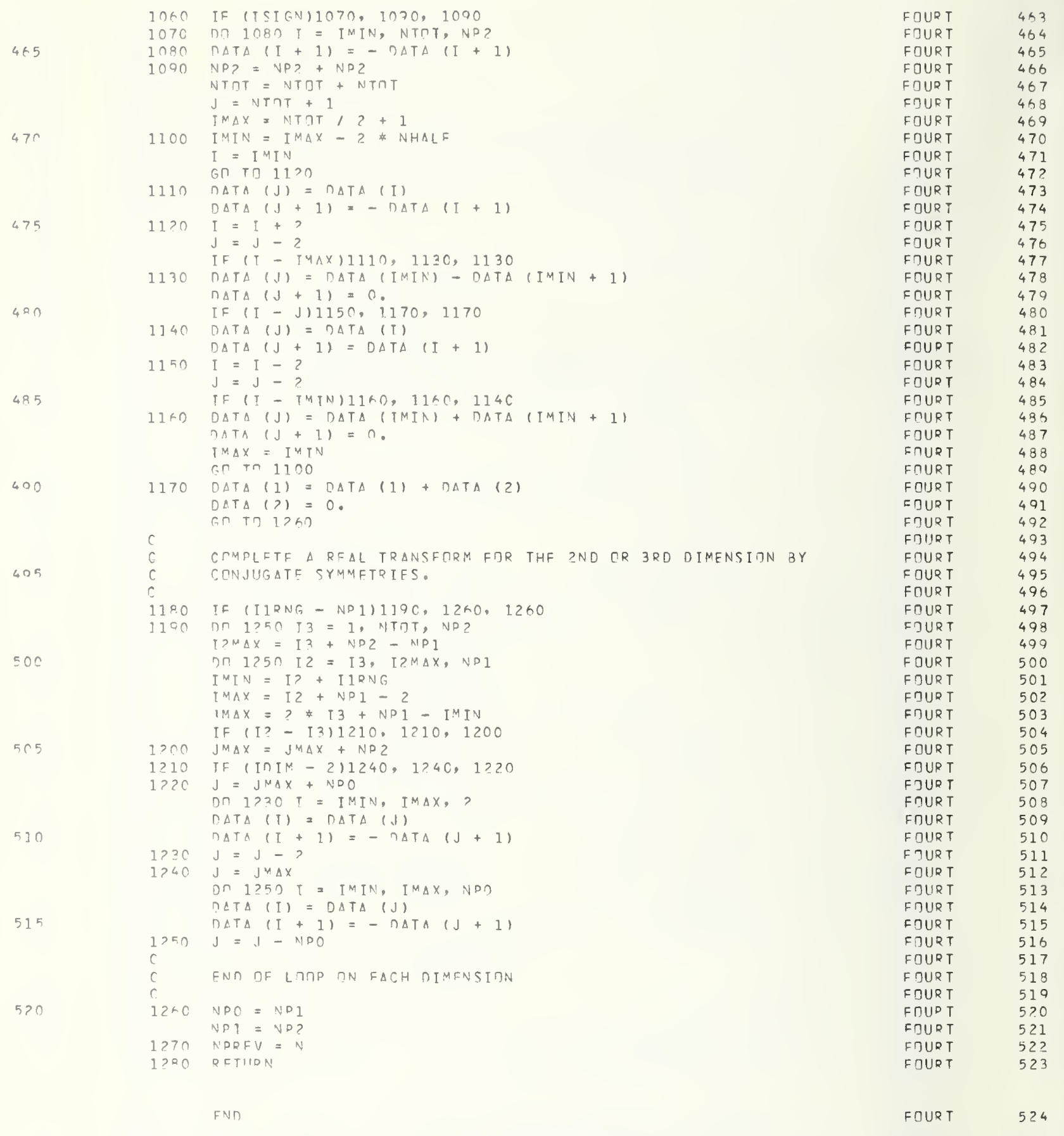




\section{A.1.11 SUBROUTINE \\ PARAB (FOD ,DOL, BLOCK, DFOCUS, ACOSE, ACOSH, THETA, ETHETA, EPHI)}

\section{PURPOSE:}

This subroutine calculates the E- and H-plane far electric field for an axially defocused, circularly symmetric, paraboloidal reflector antenna at a specified angle from the axis.

\section{ARGUMENTS:}

FOD is the focal length to diameter ratio for the reflector.

DOL is the diameter of reflector in wavelengths.

BLOCK is the fractional diameter blockage.

DFOCUS is the amount of axial defocusing in wavelengths (positive direction corresponds to feed beyond focal point).

ACOSE is the E-plane aperture 117 umination factor.

ACOSH is the H-plane aperture illumination factor. (NOTE: See discussion of POMODL for a more complete discussion of ACOSE and ACOSH.)

THETA is the angle from axis at which field values are desired in degrees. ETHETA is the electric field in E-plane.

EPHI is the electric field in H-plane.

\section{DISCUSSION:}

This and associated subroutines EPINT, ETINT, QATRC, and BESFUN were written by Professor W. V. T. Rusch of the University of Southern California. This discussion is intended to indicate the computations performed and is not a detailed description of the operation of the subroutines.

The subroutine uses PO as discussed in section 3 of the report. It is assumed that the antenna is rotationally symmetric, thus allowing very rapid execution.

Aperture illumination may be of three types: uniform, dipole, or $\cos ^{p} \theta^{\prime}$, where $\theta^{\prime}$ is the angle from the axis of the feed. These are selected with parameters ACOSE and ACOSH, and the E- and H-plane tapers are independently specified.

The integration is performed by subroutine QATRC. This subroutine has error flags which are set when the desired accuracy is not achieved either because of accumulated round-off errors or because the integration range could not be sufficiently subdivided. PARAB prints an error message indicating the type of error. These errors occur at larger values of THETA. Care should be taken to delete any far-field points known to be in error. 
This subroutine requires that functions ETINT, EPINT, and subroutines QATRC and BESFUN be supplied. In addition, library functions ATAN, COS, SIN, ATAN2, CEXP, SQRT, CABS, and inline functions CMPLX and ABS are employed. 
1

5

10

15

$>0$

25

30

35

$4 \mathrm{C}$

SUAROUTINF PAPABIFCD, CCL, BLDCK, NFOCUS, ACOSE, ACCSH, THETA, ETHETA, EPHPARAB * I)

PADIATICN PATTFRNS FRMM A DEFICIISFD PARABCLCID

$P \triangle R \triangle R$

PRDGRAMMER - W.V.T. RUSCH

$P \triangle R A B$

I6 MAJ 1074

MODIFTEN 1? MAY 1976

COMPLEX $\triangle(I X(11), R C M B, C M R L X, A I, D I, E T H E T A, E P H I$

C. OMMIN/OATA/FחL, P I, SINT, COST, DFOCSS, $\triangle C D S E E, \triangle C C S H H$

$P \triangle R A B$

PAPAB

$P A R A B$

EXTFDNAL FTTNT, EPINT

$P A P A B$

$P \triangle R A B$

OFOC SS = DF OCUS

$\triangle C \cap S F F=\triangle C \cap S E$

$\triangle C \cap S H H=\triangle C \cap S H$

$P I=4 . \cap * A T \Delta N(1.0)$

D $T P=P I / 180.0$

$P A P A B$

$P A R A B$

$P A R A B$

$P A R A B$

$P A R A B$

$P$ TO $=180.0 / P I$

$F C L=F \cap \cap+D D L$

$\Delta=2.0 * \triangle T A N(4.0 * F O D)$

$I F(R L \cap C K . L T .0 .0001) \quad R=P I^{-}$

$I F(R L O C K . G E \cdot C . C C O I) B=2.0 * \Delta T A N(4.0 * F O D / B L C C K)$

$P \triangle R A B$

$R A R A B$

PARAB

$P A R A B$

$P A R A B$

PAR\&B

COST = COS (THFTA DTR)

SINT = SIN(THFTA*DTR)

C. $\triangle L L O A T O C(A, R, 1 . O C-C 2,11, F T I N T, R \cap M R$, IER, $A U X)$

$P A R A B$

$P A R A B$

PAR $\triangle B$

IF (IER.EO. I) PPINT 1000

IF (IFR EQ.?) PPINT 1010

ETHETA $\approx C M P L \times(0.0,1.0) * 2.0 * P I * F O L * R O M B$

CALL OATRC (A, A, I.OF-C3, II, EPTNT, RTME, IER, $\triangle U X)$

IF (IFQ .FQ.2) PRINT 2010

IF (IFR. $F 2.1$ ) PPINT 2000

EPHI $=C M P L \times(0.0, I .0) * 2.0 * P I * F \cap L * R O M B \quad P A R A B$

RETIIRN

$P A R A B$

$P A R A B$

$P A P A B$

$P A R A B$

$P A R A B$

$P A R A B$

$P A P A B$

$\mathrm{C}$

1000 FIRMATI REDIIIRFD ACCURACY NOT ACHIFVED IN E-PLANF DHF TO ROUNDINPAPAR

IG FDROPS.*)

$P A R A B$

ICIO FRPMAT(* RFQUIPEN ACCUPACY NOT ACHIEVED IN E-PLANE DUE TI INSUFFIPARAB

ICIFNT NUMBER IF TNTFGRATIMN STEPS。*)

$P A P A B$

2000 FORMATI RFQUIRED ACCURACY NOT ACHIEVED IN H-PLANE DUE TO ROUNDINPARAB

16 FPR $\cap$ S * औ

$P A R A B$

2010 FIQMAT $\%$ REQUIRED ACCIIRACY NOT ACHIEVFD IN H-PLANE DUF TO INSUFFIPARAB

ICIFNT NUMAFD DF INTFGRATION STEPS.*) END

P ARAB

$P A R \triangle B$ 
SIIRPMIJTINF: OATRC

CIMPIFX VERSITN IF SSD-RחUTINE CATR, SEPT.7?, HS-J

$O \triangle T P C$

$D \triangle T R C$

$O \triangle T R C$

DESCPIDTION OF PARANETFRS

XL - THF LOWFR GOUNO DF THF INTERVAL.

GATRC

OATRC

XIJ - THF IJPREO RTLIND AF THE INTFRVAL.

FPS - THF UPPFR BOUND TF THE $\triangle R S T L U T F$ FRRDR.

ZATRC

QATRC

NกI M

- THF DIMFNSION DF THE AUXILIARY STORAGF ARRAY AIJX. N)IM-1 IS THE MAXIMAL NUMBFD TF BISECTIONS TF

QATRC

QATRC THF INTERVAL (XL, XIJ).

OATPC

QATRC

FCT - THE NAMF DF THF EXTERNAL FUNCTION SUBORJGRAM USED. GATRC

Y - THE PFSULTING $\triangle P R P M X I M A T I O N$ FCD THE IVTEGRAL VALUF.OATRC

IFR - $\triangle$ RFSULTING ERPMR DARAMETFR.

- AY AUXTLI ARY STOPAGF ARPAY WITH DIMENSION NOIY.

$Q A T P C$

2ATRC

PFM $1 R$ KS

FQDOR DARAMFTER IFD IS CMDFD IN THF FOLLOWING FCRY

IFR=? - IT ASS PISSIBLF TD RF $\triangle C H$ THF PEQUIRED ACCURACY. NT ERRDR

IFR=1 - IT IS IMPTSSIBLF T? PFACH THF REQUIRED ACCURACY PFCAUSE DF R TIINDING FRRDRS.

$\triangle \triangle T R C$

Q $\triangle T R C$

$O A T R C$

QATRC

QATRC

$\triangle \triangle T Q C$

IF $P=$ ?

- IT WAS IMROSSIRLF TO CHECK ACCURACY BFCAUSE NDIM IS LFSS THAN 5, TR THE REQUIRED ACCURACY COULD NOT BF PEACHED WITHIN NDIM-1 STEPS. NDIM SHCULD BF

$O \triangle T R C$ INCRFASFD.

SIJRR IITINFS $\triangle N O$ FUNCTION SURRDIGRAMS RFOUIRED

THF FXTFRNAL FUNCTION SURPROGRAM FCT(X) MUST AE CODED SYY

THE ISSER. ITS ARGUMENT X SHIUID NDT RE TESTRTYED.

METHกD

EVALIJATIחN TF Y IS JUNF BY MEANS TF TRAPFZDIDAL RULE IN

CINNFC,TIMN WITH RTMBERGS RPINCIRLE. TN RETURV Y CMNTAINS THF REST PISSIRLF APPR RXIMATION DE THF INTEGRAL VALUF AND VFCTMR ALIX THF UPWARD DIAGMNAL CF RTMBERG SCHEME.

CATRC

QATRC

$Q A T R C$

$\triangle \triangle T R C$

$\triangle A T P C$

QATRC

QATRC

QATRC

QATRC

QATRC

QATRC

$O A T R C$

QATRC

П MMPINFNTS $\triangle U X(I)(I=I, 2 \ldots$ IFND, WITH IEND LFSS THAN DR QATRC FQUIAL TO MIIMI RFCMMF ARPR IXIMATIONS TI INTFGRAL VALUF WITH OATRC TFRRFASING ACCLRACY ZY MULTIRLICATION WITH (XU-XL).

FחD QFFFOFNCF, SFF

(1) FILIRRI, DAS VFRFAHREN VIN RCMBFOG-STIEEEL-RAUER ALS SPFZIALFALL DFS ALLGFMEINFN PRINZIPS VCN RICHARISON, MATHFMATIK-TFCHNIK-WIRTSCHAFT, VחL.11, ISS.2 (1954), $P R .49-54$

OATRC

QATRC

$Q \triangle T R C$

QATPC

$Q \triangle T P C$

$O \triangle T R C$

(?) BAUFP, ALGOPITHM tO, CACM, VOL.4. ISS.6 (1961), RP.?55. OATRC

QATRC

- QATRC

$\triangle \triangle T R C$

QATRC

QATRC

CTMPLFX FCT, Y, SM, $\triangle U X(N \cap I M)$

$\triangle T R C$

$Q \triangle T R C$

$H=X U-X L$

1 I $F(4) ? .10$. ?

Q $\triangle T R C$

CATRC

QATRC

NOIM IS GOFATER THAN I AND H IS NIT FOUAL TCO.

$2 \quad H M=H$

$E=F R S / A R S(H)$

$\Gamma F L T ?=$ ?

$\mathrm{P}=1$.

$J \mathrm{~J}=1$

D) $7 I=?$, NDIM

$Y=A \| \times(1)$

NFLT $1=\cap F L T$ ?

$u n=\mu \omega$

QATRC

OATRC

$2 \triangle T P C$

QATRC

QATRC

Q $\triangle T R C$

Q $\triangle T R C$

$\triangle A T R C$

$\triangle \triangle T R C$

DATRC 
on

95

จา

05

$10 n$

105

110
$H H=.5$ A H H H

$D=.5 * D$

$X=X L+H 4$

$\varsigma M=(n, 0,0)$

$n \Gamma 3 \mathrm{~J}=1, \mathrm{~J} J$

$S N_{1}=S M+F C T(X)$

$3 x=x+40$

$\Delta(1 X(I)=.5 * \Delta U X(I-1)+P \neq S M$

C. ANFW APPROXIMATITN CF INTEGRAL VALIIF IS COMPUTEO BY MEANS $3 F$ TRAPFIDIDAL RIILE.

c

STADT TF PTMRERGS EXTPADOLATITN MFTHOD.

$0=1$.

$\mathrm{I} \mathrm{J}=\mathrm{I}-1$

$\Gamma+4 \mathrm{~J}=1, \mathrm{~J}$

$I I=I-J$

$0=0+0$

$c=c^{x}+2$

$4 \Delta 11 \times(I I)=\Delta 1) \times(I I+1)+(\Delta U) \times(I I+1)-\Delta 11 \times(I I)) /(0-1$.

FNO OF RIMRFRG-STFP

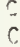

$\cap F(T)=C A R S\{Y-A U \times(1))$

IF $(T-5) 7,5,5$

5 IFIDELT?-5) I0,10, K

* IF(חELT?-חELT1) 7.1.1.1]

$7 \quad \mathrm{~J}=\mathrm{J} \mathrm{J}+\mathrm{J} \mathrm{J}$

- $Y F R=$ ?

9 $Y=H * \Delta \| \times(1)$

DETURN

10 I $F P=0$

G) $T \rightarrow 9$

11 I $F R=1$

$Y=H$ * $Y$

PFTUPN

FND

\begin{tabular}{|c|c|}
\hline$D \triangle T R C$ & 78 \\
\hline$Q \triangle T R C$ & 79 \\
\hline Q $\triangle T R C$ & 80 \\
\hline$O \triangle T R C$ & 81 \\
\hline$Q \triangle T R C$ & 82 \\
\hline QATRC & 83 \\
\hline$O \triangle T R C$ & 84 \\
\hline$O \triangle T R C$ & 85 \\
\hline CATRC & 86 \\
\hline OATRC & 87 \\
\hline$D \triangle T R C$ & 8 ค \\
\hline$O \triangle T R C$ & 89 \\
\hline$D \triangle T P C$ & 90 \\
\hline$O A T R C$ & 91 \\
\hline Q $\triangle T R C$ & 92 \\
\hline OATRC & 93 \\
\hline$D A T R C$ & 94 \\
\hline$D \triangle T R C$ & 95 \\
\hline OATRC & 96 \\
\hline OATRC & 97 \\
\hline Q $\triangle T P C$ & 98 \\
\hline DATRC & 99 \\
\hline$O \triangle T R C$ & 100 \\
\hline$D A T D C$ & 101 \\
\hline Q $\triangle T R C$ & 102 \\
\hline Q $\triangle T R C$ & 103 \\
\hline$Q \triangle T R C$ & 104 \\
\hline OATRC & 105 \\
\hline$O \triangle T R C$ & 106 \\
\hline Q $\triangle T R C$ & 107 \\
\hline$Q \triangle T R C$ & $10 B$ \\
\hline QATRC & 109 \\
\hline$\triangle A T R C$ & 110 \\
\hline$O \triangle T R C$ & 11 \\
\hline$D \triangle T P C$ & 112 \\
\hline
\end{tabular}


COMPLEX FUNCTIIN ETINT $(X)$

COMMONIDATA/FCL, PI, SINT, COST, DFOCUS, ACOSF, $\triangle C O S H$

5

C

NOTF THAT COSLP

MPLFX CEXP, CMPLX,AI, R1, HX

$\operatorname{SINX}=\operatorname{SIN}(X)$

$\cos x=\cos (x)$

QHIMVL $=2.0 * F \Pi L /(1.0-C O S X)$

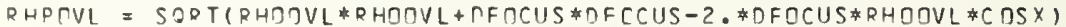

$R 1=$ RHODVL*SINX

$R 2=$ NFOCUS - RHCOVL $*$ COSX

$X P=P I-\triangle T A N Z(R 1, R 2)$

CSPMXP $=$ R2/RHDOVL

$F A Z F=2.0 * P I *(R H O C V L * C \cap S X * C O S T-Q H P Q V L)$

RFTA $=2.0 * P T * R H D C V L * S I N \times$ SINT

IF (BETA. GT.0.0) GC TC?

$A F S S O=1.0$

RFSSI $=0.0$

Q F S S $=0.0$

Gח TO 3

? CALL RFSFUN (BFT $\triangle, B J, 4)$

RFSSO = RJ (1)

RFSSI $=R \mathrm{~J}(2)$

AFSS2 $=$ aJ (3)

3 CONTINIIF

IF (ACDSF.GF. $(-100.0)$ GT TD 20

$\Delta 1=? .0 /(1.0+C S P M \times P)$

$01=-41$

GO TI 50

20 IF $\triangle \triangle C D S E . G F \cdot 0 . C) B O$ TO 40

$A 1=C S P M X P$

$01=-1.0$

60 Tก 50

$\Delta 1=\operatorname{CSPMXP**ACOSF}$

$01=-\operatorname{CSPM} \times P * \triangle C D S H$

50

CCNTINIJE

$H X=($ RHOTVL/RHPCVL) * $(A 1 * C O S T$ (RESSO-BFSS2)-DI*COST*(BESSO+BESS2)*FTINT

\#SIN $(X P-(x / 2.0)) / S I N(X / 2.0)-2.0 * C M P L \times(0.0,1.0) * \Delta I * R E S S I * C O S(x / 2.0) / E T I N T$

* SIN $(x / 2.0) * S I N T)$

$H X=H X /(1.0-C O S X)$

ETINT $=H X * S I N X * C E X P(C M D L X(0.0, F A Z F))$

RETIJRN

END
ET INT

ETINT

ETINT

ETINT

ETINT

FT IN T

ETINT

ETINT

E T IN T

ETIN

ETINT

ETINT

ETINT

ETINT

ETINT

ET INT

ETINT

ETIN T

E T INT

ET INT

ET INT

ETINT

ETINT

ETINT

ET INT

ET IN T

ETINT

ET IN T

ETINT

ETINT

ET I NT

E TINT

ETINT

ETINT

E T IN T

ETINT

ETINT

ETINT

ETINT 
1

5

10

15

20

25

30

35

40
C MMPLFX FUNCTION FPINT $(X)$

CDMMON/DATA/FCL, DI, SINT, COST, DF C US, $\triangle C O S F, \triangle C O S H$

DIMFNSION BJ $(1000)$

NITE THAT COS(PI-XP) = (PFDCUS-RHI COSX)/RHOPPIMF = 22/RHPOVL

CCMPLFX CEXP, CMPLX, $1, D 1, H X$

$\operatorname{SIN} X=\operatorname{SIN}(x)$

$\cos x=\cos (x)$

RHOCVL $=? .0$ FOL $/(1.0-C \cap S X)$

PHPDVL = SQRT (RHMIVL *RHOOVL+DFOCUS*DFCCUS-2. *DFOCUS*RHOOVL *COSX)

$R 1=$ R.MOTVL*SINX

Q $=$ DFOCUS - PHODVL $*$ COSX

$X P=P I-\triangle T A N 2(R 1, R 2)$

CSPMXD $=$ RZ/RHPRVL

$F \triangle Z F=2.0 * P I *(R H C C V L * C O S X * C O S T-R H P D V L)$

BFTA $=? .0 * P I * P H O O V L * S I N \times S I N T$

IF(AFTA.GT.0.0) GO TI 2

AESSO $=1.0$

$B E S S 1=0.0$

PESS? =0.0

Gก TD 3

2 $\triangle L L$ BFSFIIN ( $P F T A, R J, 4)$

RFSSO $=$ RJ $(1)$

RESS $1=B J(2)$

BFSS? = BJ(3)

3 CONTINIJF

[F(AT,ISF, GF. $(-100.0)) G 0$ TD 20

$\Delta 1=2.0 /(1.0+C$ SPMXP)

$D 1=-41$

$G[T] 50$

20 IE( ACOSE.GE.0.0)GO TO 40

$\Delta 1=\operatorname{CSPMXD}$

$D 1=-1 \cdot 0$

GL TT 50

40

$\Delta 1=\operatorname{CspM} \times D * \Delta \cos F$

50

$01=-\operatorname{CSPM} \times P * \# \triangle \mathrm{COSH}$

CONTI NUE

* 2.01)/ SIN $(x / 2.1)$

$H X=H X /(1.0-\operatorname{COS} X)$

EPINT $=4 X * S I N X * C F X P(C M P L X(0.0, F A Z E))$

RETURN

ENก
EPINT

EP INT

EPINT

EPINT

EPINT

EP INT

EPINT

EPINT

EP INT

EPINT

EPINT

EPINT

FPINT

EPINT

EPINT

EPINT

EPINT

EPINT

EPINT

EPINI

EPINT

EPINT

EPINT

EPIN T

EP INT

EP IN T

EP INT

EPINT

EPINT

EP INT

EP INT

EP INT

EP INT

EP I NT

EP INT

EPINT

EPINT

EP INT

EPINT

EP INT

EPINT 


\section{A.1.12 SUBROUTINE \\ $\operatorname{PLT12OR}(X, Y, X M A X, X M I N$, YMAX, YMIN, LAST , IS YMBOL, NO ,MOST)}

PURPOSE:

To make a page plot of array $Y$ versus array $X$.

ARGUMENTS:

$X=$ Array containing abscissa values of the function to be plotted.

$Y=$ Array containing ordinate values of the function to be plotted.

XMIN = Minimum abscissa value.

XMAX = Maximum abscissa value.

YMIN = Minimum ordinate value.

YMAX = Maximum ordinate value.

LAST = Number of points to be plotted.

ISYMBOL = A Hollerith variable containing the plotting symbol, e.g., to plot with the symbol "X" ISYMBOL = IHX.

NO = Number of plot on page.

MOST = Total number of plots to be made on one page.

\section{DISCUSSION:}

This subroutine produces a "quick and dirty" plot of $Y$ versus $X$ on the page printer. The size of the plotting area is $50 \times 120$ units. Multiple plots may be made on a single page. A page eject is performed before the first plot of a series is begun, but no eject is performed after completion of a series. This allows a title to be printed at the bottom of the plot. The subroutine uses inline function FLOAT. 


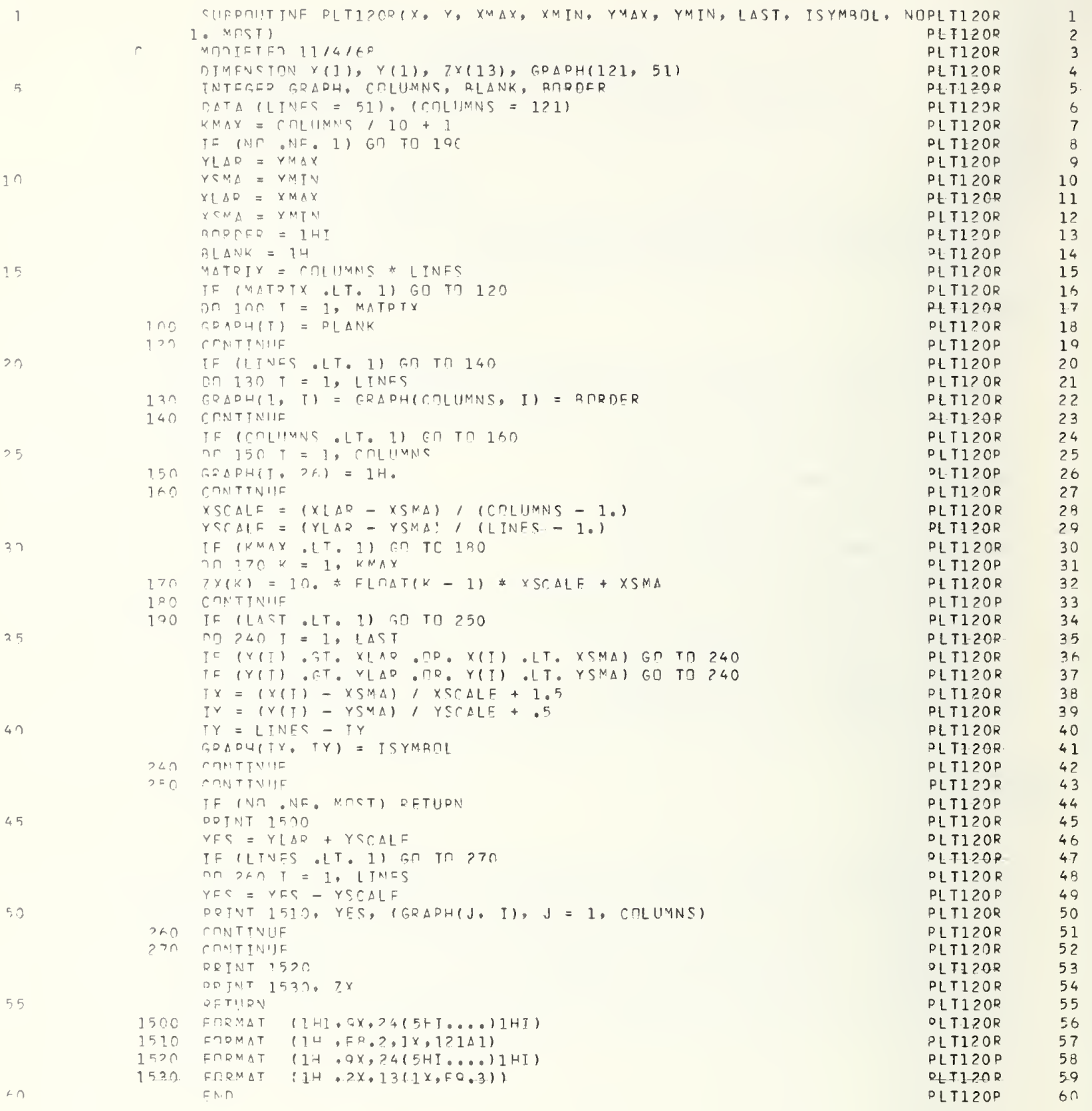


Illustrated below is a typical input card deck for program POMODL. The output produced using this deck is reproduced on the following pages.

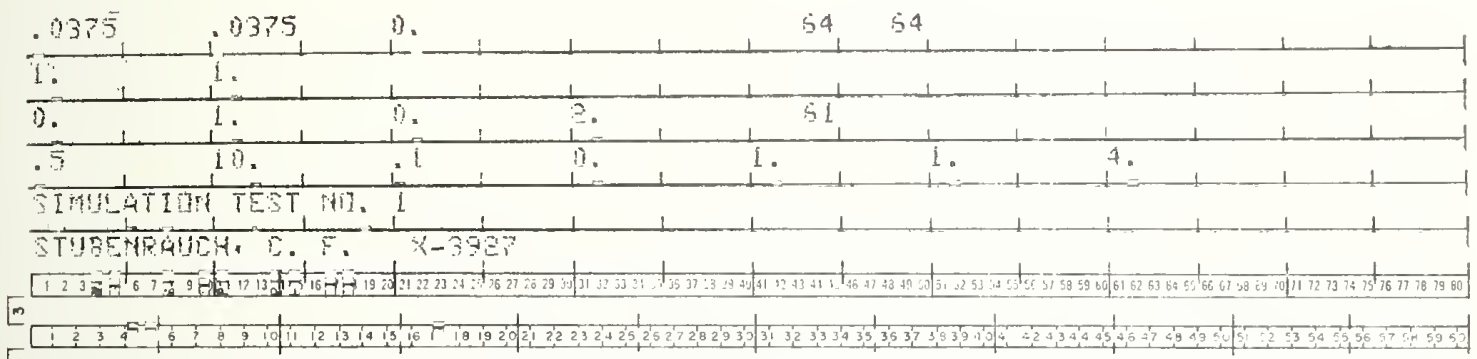

[

0010000100001000001000010000010000000000100000100000100000000001000000000010000000000

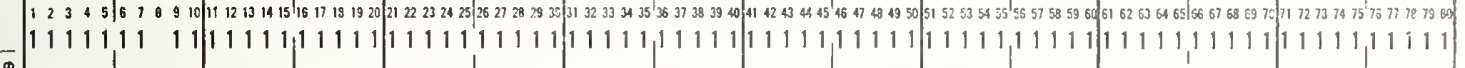




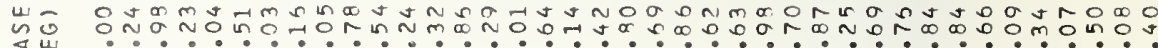

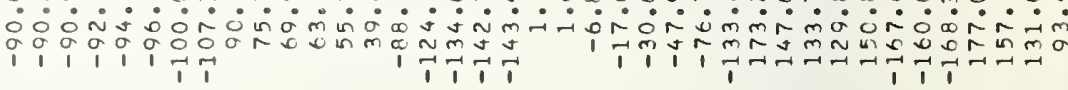

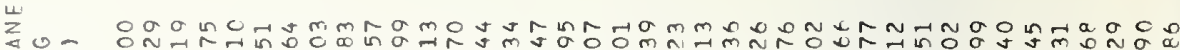

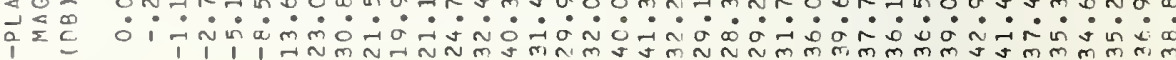
工

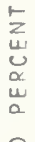

Dm DONJOmLtR N

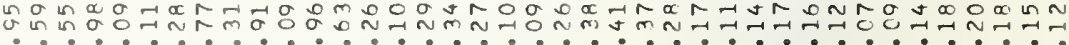
$\dot{0} \dot{0} \dot{\sim} \dot{0} \dot{\mathrm{i}}$

$\sim \overline{0}$

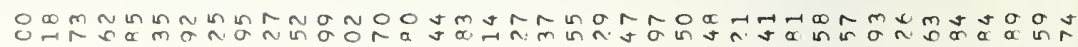
I

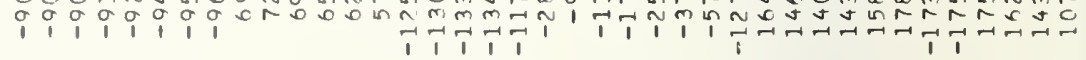

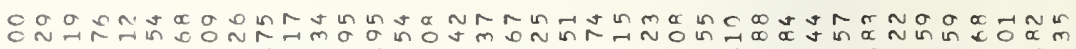

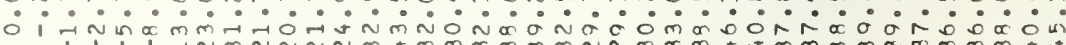

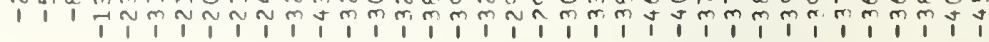

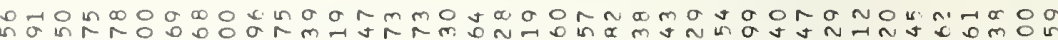
on. 000

000000000000000000000000000000000000000

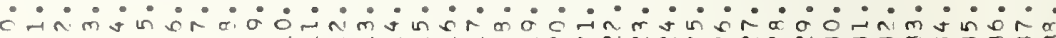




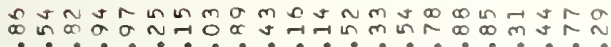

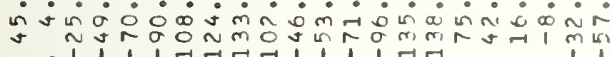

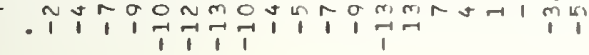

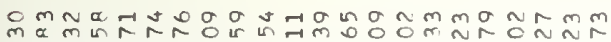

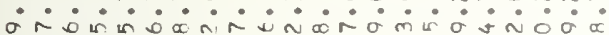

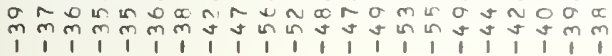

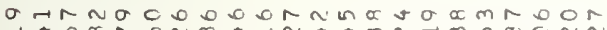

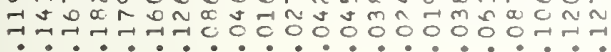

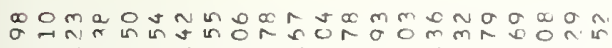

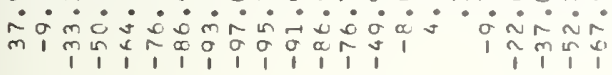

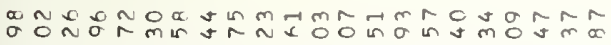

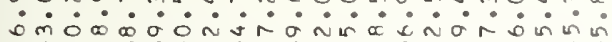

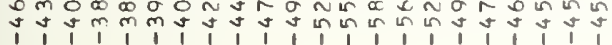

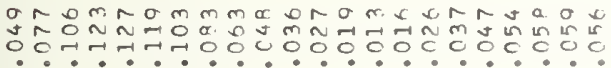

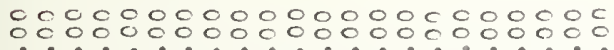

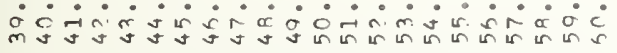




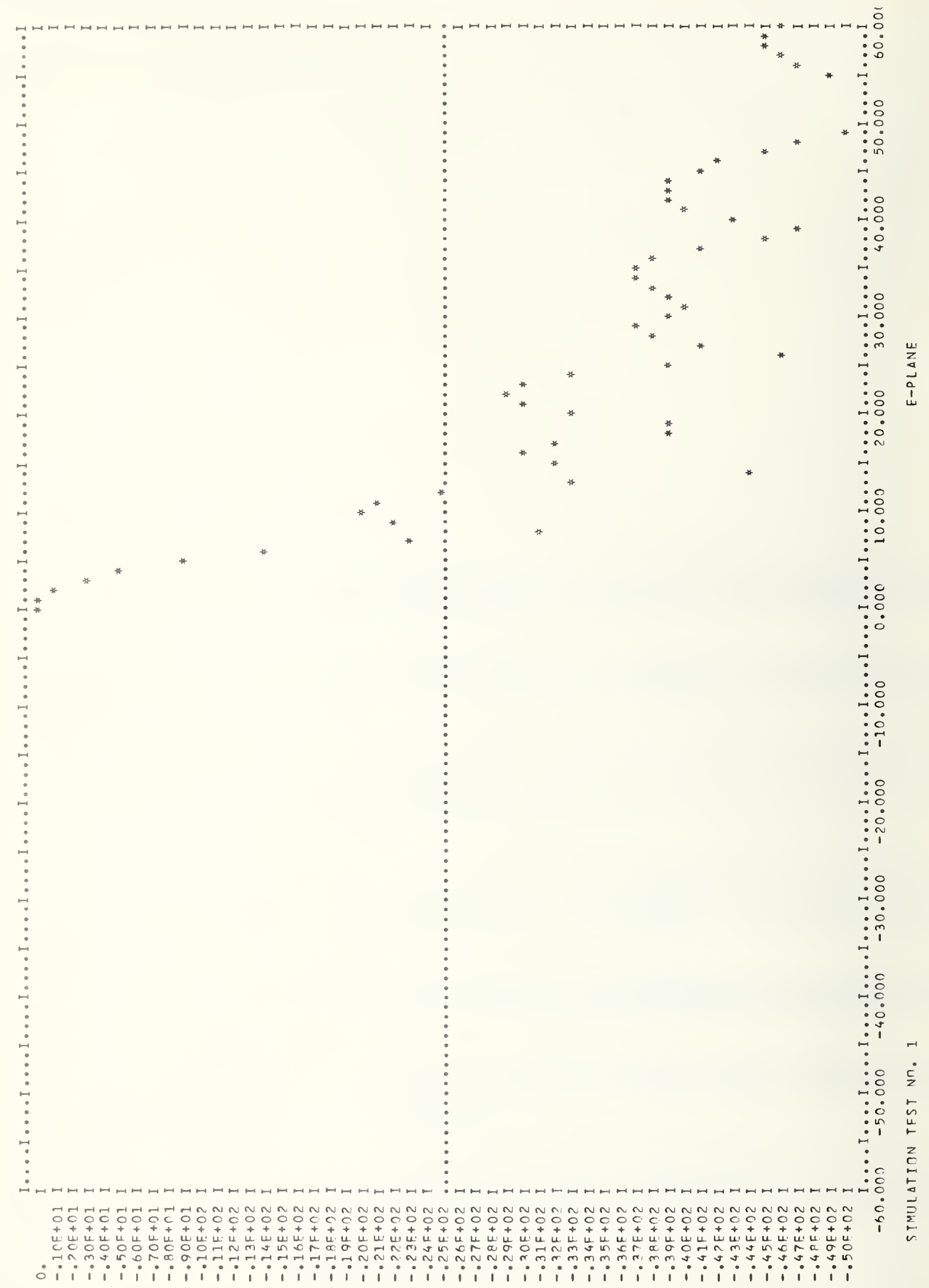




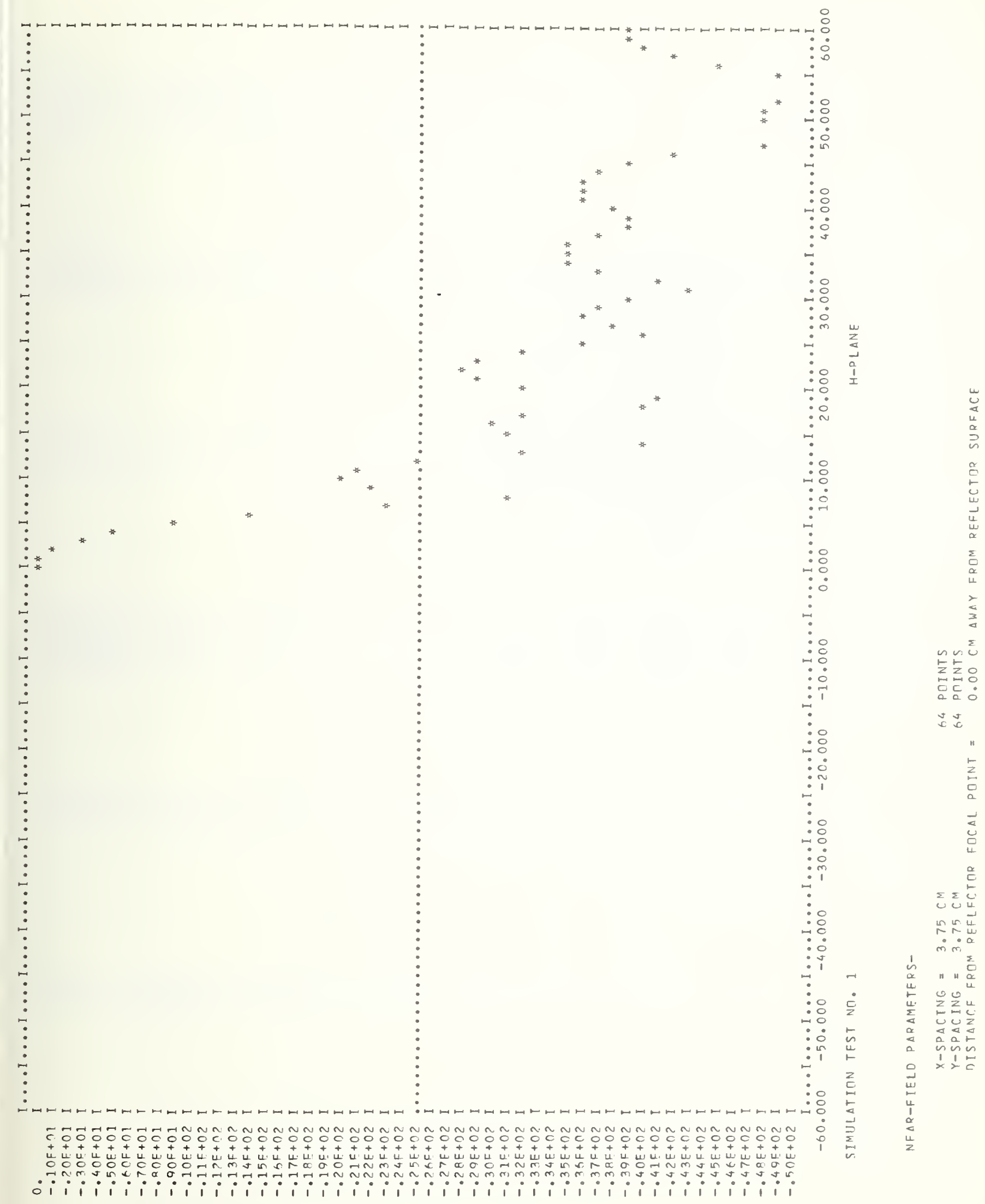




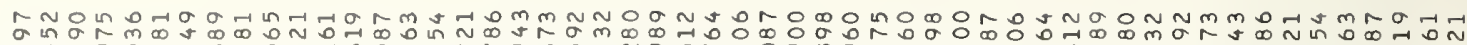

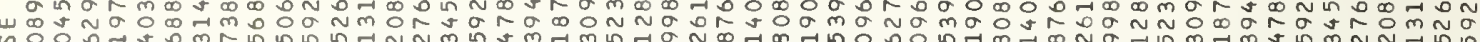

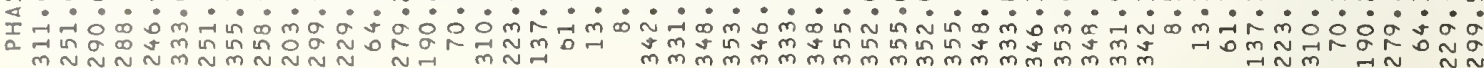

山س Z

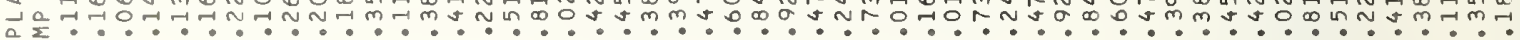
N $\stackrel{n}{\prime}$

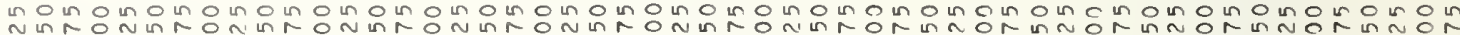

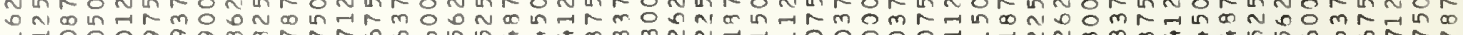

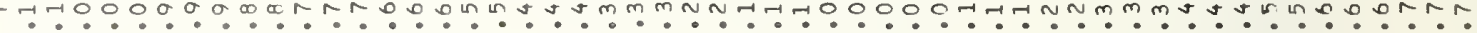
$\vec{\imath} \vec{\imath} \vec{\imath} \vec{\imath}$

Oก⿻上丨

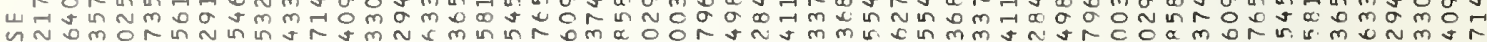

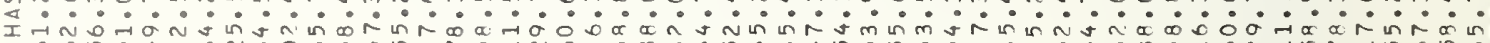
I

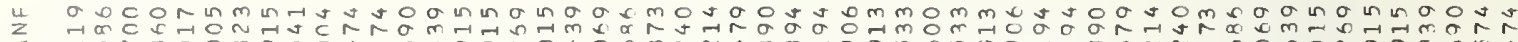

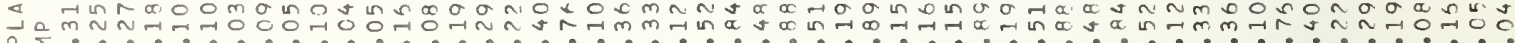
N 1

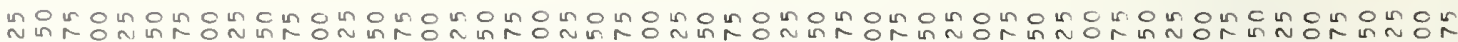

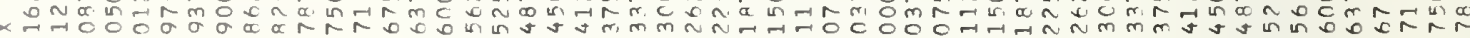

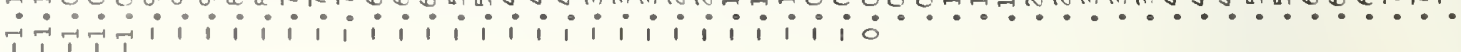




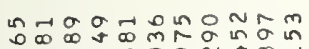

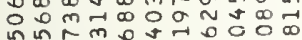
$\dot{0} \dot{0} \dot{0} \dot{0} \dot{0}-\dot{1}$

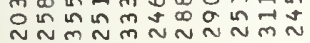

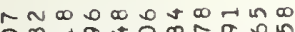
ำดั

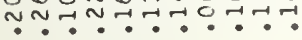

o in o n 0 in 0 m 0 in แnกำ

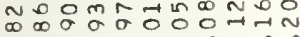
•.. - $-\dot{-i} \dot{-}$

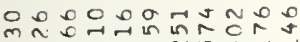

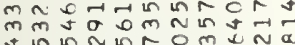

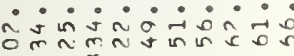
Nin

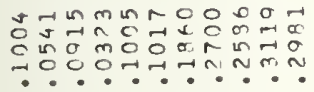

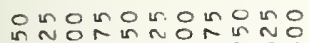

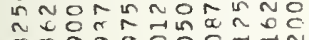

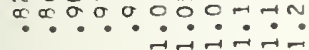




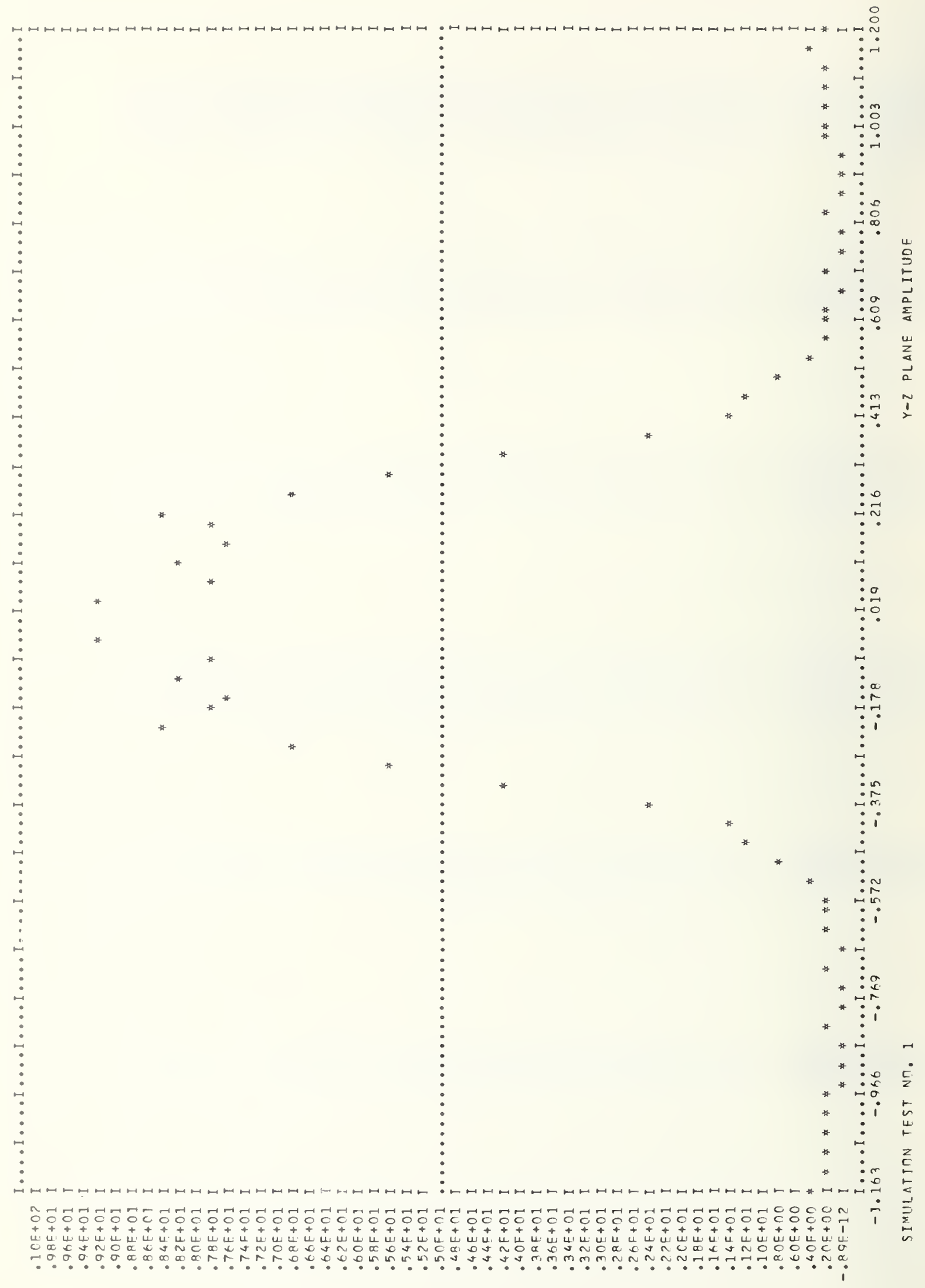




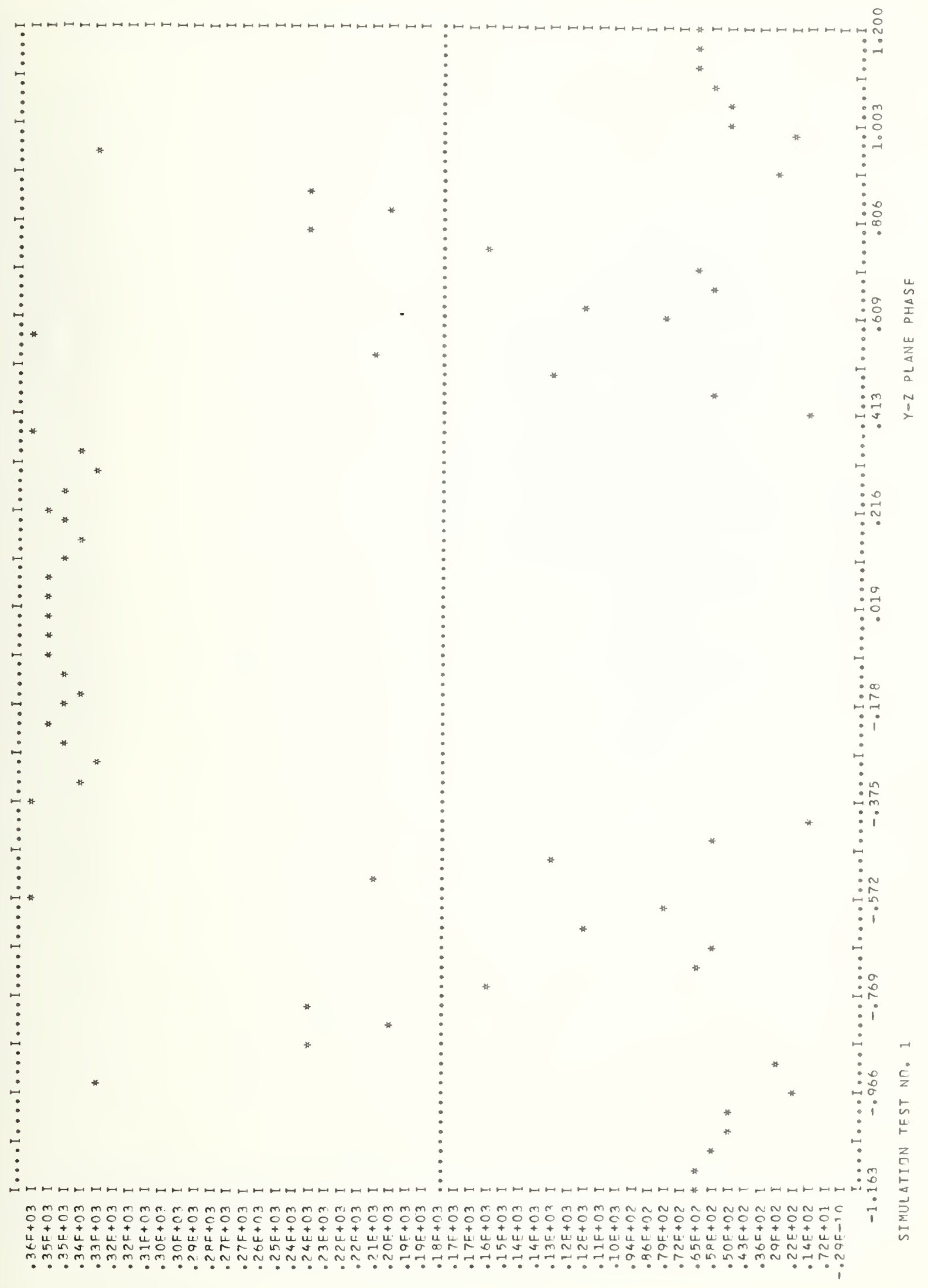




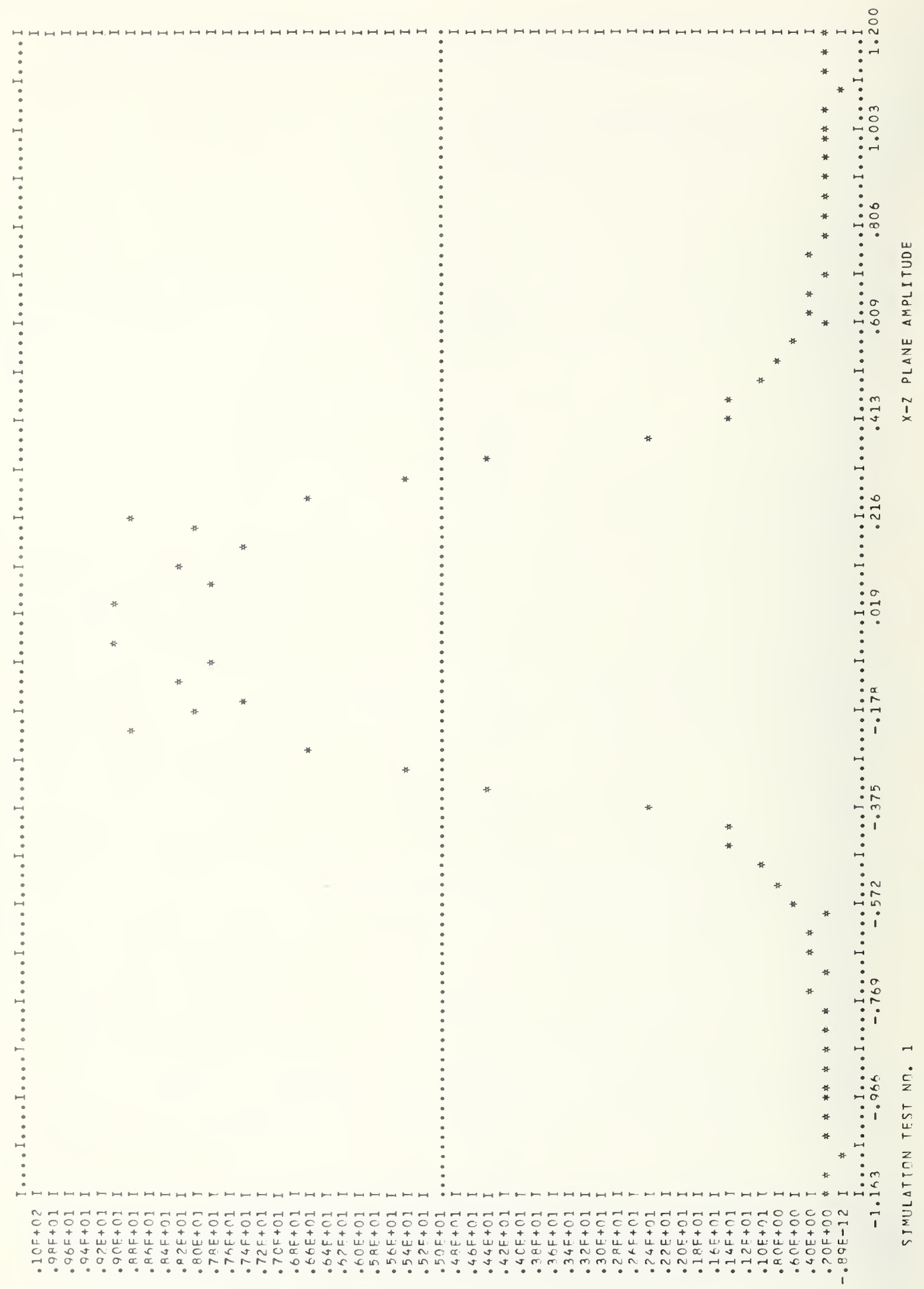




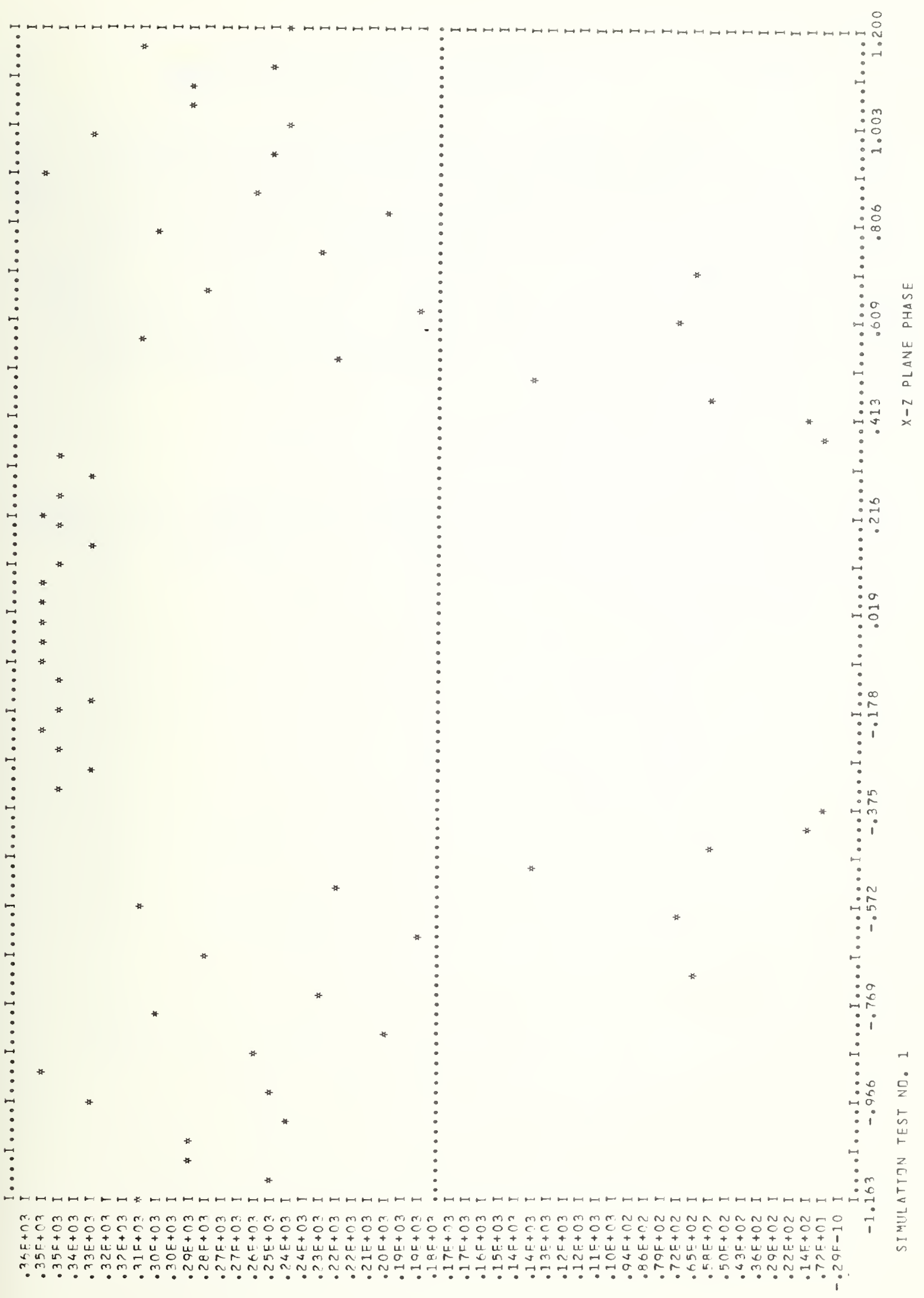


This appendix includes detailed documentation of the program which calculates coupling between two antennas given their far-field patterns. This program, as presented here, uses only a single component of the far field for each antenna, and is thus applicable only for linearly polarized antennas oriented with the major components of their polarization vectors lying in a common plane. The inclusion of the cross component in the calculation is not a difficult extension to the program. Each subroutine is individually documented except for those which are also used in PROGRAM POMODL and are discussed in Appendix A. The final section of the appendix includes a sample input deck and a sample program output.

\section{B.1 GENERAL OVERVIEW OF COMPUTER PROGRAM}

The program CUPLNF and its associated subroutines are described in detail in the following subsections. The flow chart below is presented in order to give the reader a general overview of the program package. 


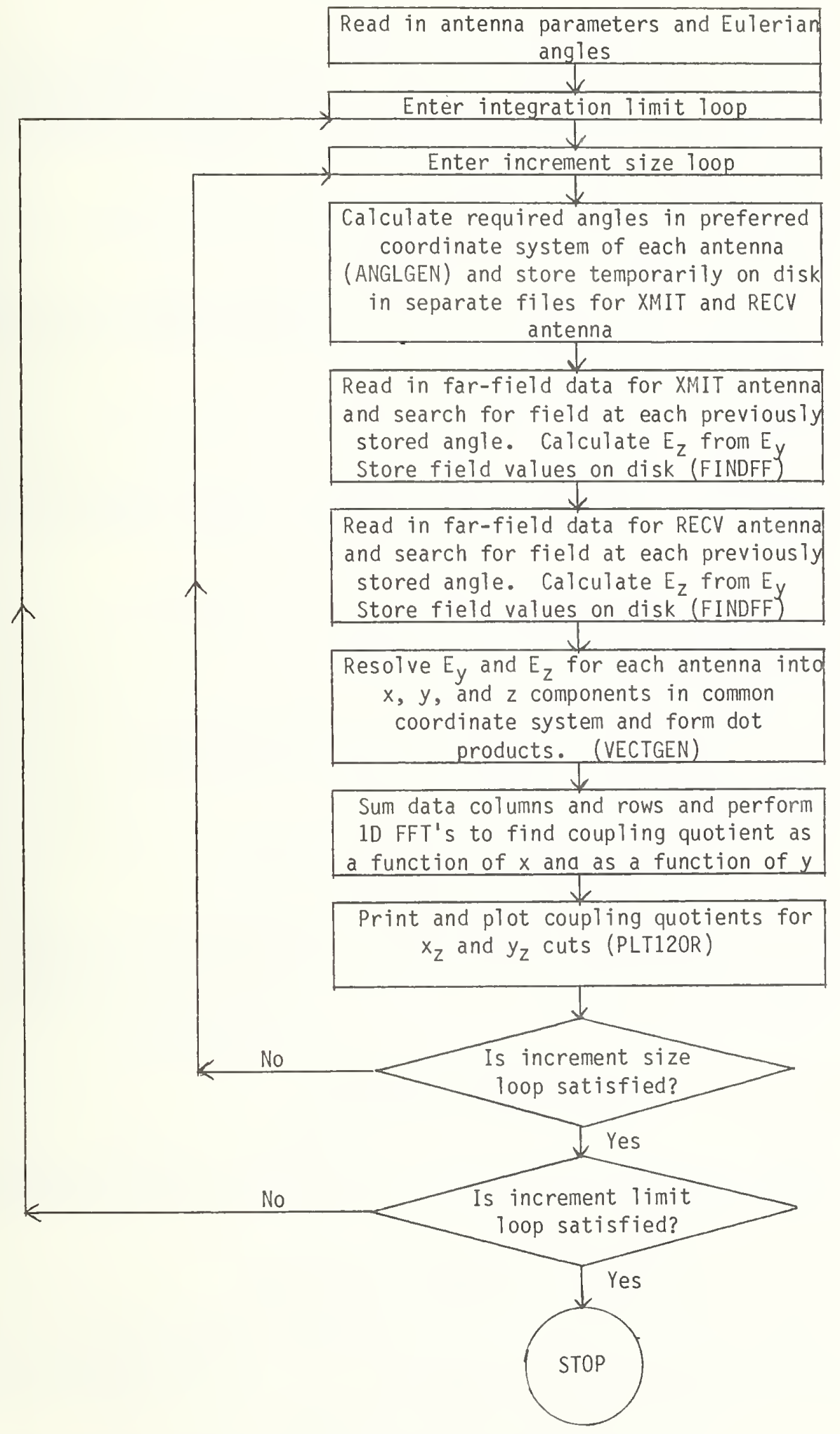


PURPOSE:

To compute and plot the mutual coupling between a transmitting and receiving antenna of arbitrary orientations and separation from the given complex far-electric-field pattern of each antenna.

METHOD:

Evaluate eq (32) of the main text along $x$ and $y$ perpendicular lines or cuts, using the fast Fourier transformation.

\section{GENERAL DISCUSSION:}

The main program divides conveniently into six subsections which list sequentially as follows:

1) General information about program,

2) Specification statements,

3) Definition and reading of input data,

4) Limits of integration and number of integration points,

5) Filling of the input matrices ( $A X$ and $A Y$ ) to the FFT FOURT, and

6) Printout and plotting.

\section{General Information about the Program}

This subsection is a self-explanatory aid providing the program user with specific definitions of the main input parameters required by the program, as well as with a general feel for what the program does.

\section{Specification Statements}

This subsection merely dimensions, equivalences, and comments the appropriate arrays, and declares the necessary complex and integer variables.

\section{Definition and Reading of Input Data}

This subsection defines and reads from data cards the input variable parameters to the program. A list of the required data cards follows:

Card 1 Col. 1-40 An alphanumeric identifier, usually the name and telephone extension of the person submitting the job. 
Card 2 Col. 1-80 An alphanumeric identifier specifying the particular case being studied.

Except where specifically noted all data on the following cards must have the decimal point explicitly specified.

Card 3 Col. 1-10 Frequency of operation in $\mathrm{GHz}$.

Co1. 11-20 Distance between origins of the reference coordinates of the two antennas in meters.

Col. 21-30 x-spacing corresponding to the near-field spacing for the transmitting antenna.

Col. 31-40 y-spacing corresponding to the near-field spacing for the transmitting antenna.

Co1. 41-50 x-spacing corresponding to the near-field spacing for the receiving antenna.

Col. 51-60 y-spacing corresponding to the near-field spacing for the receiving antenna.

Co1. 61-70 Ratio of transmitting to receiving antenna feed mode admittances.

Col. 71 Set equal to 1 if spectrum rather than far-field pattern is given

Card 4 Col. 1-10 Maximum value for plot. If this field is left blank, the scale is chosen to fill the plot page.

The remaining data on this card are integer data, and must be right justified in the field provided.

Col. 11-15 Lower index in the increment loop.

(Set equal to 1 if field is blank).

Col. 16-20 Upper index in the increment loop.

(Set equal to 1 if field is blank).

Co1. 21-25 Lower index in the integration limits loop. (Set equal to 1 if field is blank).

Col. 26-30 Upper index in the integration limits 10op. (Set equal to 1 if field is blank). 
Card 5 Col. 1-10 Transmitting antenna gain in $\mathrm{dB}$.

Co1. 11-20 Magnitude of transmitting antenna far-field pattern on boresight. (Allows for normalization of far-field pattern).

Co1. 21-30 Radius of transmitting antenna in meters.

$\begin{array}{ll}\text { Col. } 31-40 & \text { PHI } \\ \text { Co1. 41-50 } & \text { THETA } \\ \text { Col. 51-60 } & \text { PSI }\end{array}$

Co1. 61-65 NROWT - Number of rows of data in transmit antenna pattern. (Integer data right justified in field).

Co1. 66-70 NCOLT - Number of columns of data in transmit antenna pattern. (Integer data right justified in field).

Co1. 71-80 Transmit antenna pattern file identifier.

Card $6 \cdot$ Col. 1-10 Receive antenna gain in $\mathrm{dB}$.

Co1. 11-20 Magnitude of receive antenna far-field pattern on boresight. (Allows for normalization of far-field pattern).

CoT. 21-30 Radius of receive antenna in meters.

$\begin{array}{ll}\text { Col. 31-40 } & \text { PHIP } \\ \text { Col. 41-50 } & \text { THETHP } \\ \text { Col. 51-60 } & \text { PSIP }\end{array} \mid$ - Eulerian angles of reoriented receive antenna.

Co1. 61-65 NROWR - Number of rows of data in receive antenna pattern. (Integer data right justified in field).

Co1. 66-70 NCOLT - Number of columns of data in receive antenna pattern. (Integer data right justified in field.)

Co1. 71-80 Receive antenna pattern file identifier.

Card 7 Col. 1-20 GAMT - Transmit antenna reflection coefficient.

(Real part 1-10, imaginary part 11-20).

Col. 21-40 GAMR - Receive antenna reflection coefficient.

(Real part 21-30, imaginary part 31-40). 


\section{Co1. 41-60 GAML - Receiving load reflection coefficient. (Real part 41-50, imaginary part 51-60).}

\section{Limits of Integration and Number of Integration Points}

In the analysis of the main text, it is shown that only the far-field pattern within the sheaf of angles mutually subtended by the two antennas is necessary to accurately compute the coupling between the antennas. These reduced limits of integration artifically bandlimit the coupling and thus increase the integration increments required by the sampling theorem. In al1, the number of integration points is drastically reduced. This subsection of CUPLNF computes a maximum solid angle mutually subtended by the antenna and translates this information into specific limits of integration for $k_{x}$ and $k_{y}$. In addition, the integration increments and subsequently the number of integration points in the $x$ and $y$ directions are also obtained in this subsection.

\section{Filling of the Input Matrices (AX and AY) to the FFT FOURT}

Now that the previous subsection has computed the points and limits of integration, the far-field patterns of each antenna must be retrieved from input files at the specified points of integration. These far-field arrays are inserted as input into the FFT FOURT in order to compute the coupling quotient from eq (32) of the main text. The subroutine FINDFF, documented separately, takes the required array of far-field integration points (directions) searches the input files containing the far field for the value of far field in the required directions, and outputs the array of far-field values to be used eventually by FOURT.

Before calling FINDFF, the program must calculate the far-field directions corresponding to the integration variables $k_{x}$, $k_{y}$ in the integral (summation) of eq (32). This is accomplished through the subroutine ANGLGEN, which is documented separately.

After the far-fields are obtained from FINDFF, their dot product must be determined as eq (36) demonstrates explicitly. This scalar product is accomplished through the short subroutine VECTGEN, which has been documented separately from CUPLNF.

The dot products of the far-fields found from VECTGEN are appropriately placed in two arrays, $A X$ and AY, from which the FFT subroutine FOURT computes the near-field coupling quotient along two mutually perpendicular $x$ and $y$ cuts. 


\section{Printout and Plotting}

This subsection simply prints and plots the magnitude of the coupling quotient (i.e., coupling loss ratio) along the mutually orthogonal $x$ and $y$ cuts which lie normal to the line of separation. Because of the possible lack of a common plotting system, curves are made using a general purpose page printer subroutine (PLT120R). The coupling is plotted in the $x$ and $y$ directions over a distance approximately equal to twice the sum of the diameters of the two antennas.

\section{SYMBOL DICTIONARY:}

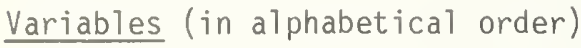

ABL = Intermediate variable for defining the range of $k_{x} / k$ and $k_{y} / k$. The range of $A B L$ beyond XKLIM is zero filled.

ACLCUT $\quad$ A real array used to store the magnitude of the coupling quotient along $\mathrm{XO}$ and Y0 perpendicular axes or cutter.

$A X, A Y=$ Complex arrays used to store first the coupling far-field product then the coupling quotient $\mathrm{along} X O$ and $Y 0$ cuts, respectively.

$(A 1, A 2),(B 1, B 2)=$ The limits of integration of $k_{x} / k$ and $k_{y} / k$, respectively.

BFAC $\quad$ Variable which adjusts the integration increments, and should be approximately 1 or 2; making BFAC larger tests whether convergence has been reached.

CEE

$=$ Speed of light in gigameters per second $=.2997925$.

COEF

= The coefficient of the summation in eq (32) of the main text with the exponential factor omitted.

CUPLDB = Coupling quotient for two antennas expressed in $\mathrm{dB}$.

$\mathrm{C} 1, \mathrm{C} 2$

$=$ The $k_{x} / k$ and $k_{y} / k$ increments, respectively, $i . e .,\left(a_{1}+a_{2}\right) / N_{1}$ and $\left(b_{1}+b_{2}\right) / N_{2}$ in eq $(32)$.

DATA = Array containing far-field pattern of transmitting or receiving antenna, used in SUBROUTINE FINDFF and included here for storage allocation purposes only.

DIAMR, (DIAMT) = Twice the larger of RADR (RADT) or WAVELGTH of the receiving (transmitting) antenna.

DIAMSUM = DIAMR plus DIAMT.

DKOK

= The approximate $k_{x} / k$ and $k_{y} / k$ integration increments, i.e., $N 1 \approx(A 1+A 2) / D K O K$ and $N 2 \approx(B 1+B 2) / D K O K$.

DLX,DLY = Subsequent labels for (DLXR and DLXT), (DLYR and DLYT).

$\mathrm{DLXR}, \mathrm{DLXT} \quad=x$ - increment which corresponds to the $k_{x}$ increment of the receiving and transmitting antenna, respectively.

DLYR,DLYT = $y$ - increment which corresponds to the $k_{y}$ increment of the receiving and transmitting antenna, respectively. 
DPHI, DPHIP, DPSI, DPSIP,

DTHETA, DTHETAP

$=$ The Eulerian angles PHI, PHIP, .....DTHETAP expressed in degrees rather than radians.

DTR = Degree to radian conversion factor.

$D X, D Y$

= The increments in $X 0$ and $Y 0$, respectively, over which the coupling quotient is computed by the FFT.

ETOER

= Ratio of characteristic admittance of the transmitting antenna feed mode to the characteristic admittance of the receiving antenna feed mode.

FDOTFP

$=$ The dot product of the complex far-electric-field pattern of the two antennas.

FFRMX, FFTMX = Magnitude of unnormalized far-field pattern at THETA $=0$ for the receiving and transmizting antennas, respectively.

FMM

= The mismatch factor, 1/(1-GAMR•GAML) in the right, receiving antenna.

FREQ

$F X, F Y, F Z$

= Frequency in $\mathrm{Hz}$.

= The complex rectangular components of far electric field in the preferred coordinate system fixed in the left, transmitting antenna. (FX and FY are also used later in the program as intermediate complex variables.)

FXP,FYP,FZP = The complex rectangular components of far electric field in the preferred coordinate system fixed in the right, receiving antenna.

FXR, FYR, FZR = The complex rectangular components of the far electric field of the right receiving antenna in its mutual coupling coordinate system.

FXT,FYT,FZT = The complex recangular components of the far electric field of the left, transmitting antenna in its mutual coupling coordinate system.

GAINR,GAINT = Gain in $\mathrm{dB}$ of receiving and transmitting antennas, respectively.

GAML,GAMR, GAMT = Reflection coefficient of receiving load, receiving antenna, and transmitting antenna, respectively.

HEAD = Integer array identifier for case under study.

IBFAC $\quad$ Loop index for varying BFAC.

ID(I) = Integer array (with index I) identifier for programmer's name and one extension.

IDAYHRR, IDAYHRT = File identifier for receiving and transmitting antenna data, respectively.

ISCL = Integer indexer for conditional statements.

I SPECT

= Spectrum flag. Set equal to 1 if spectrum rather than far-field patterns specified.

IXLIM = Loop index for varying XLIM.

$\mathrm{J1,}, \mathrm{J2}$

$=$ Dummy loop indices used in the filling of the AX, AY coupling product arrays, and later in the printout statements.

$\mathrm{L} \quad=$ Dummy index for write and read statements.

M1,M2 = Dummy loop indices used in the multiplication of the sum in eq (32) by the preceeding factors.

NBF1, NBF2 = Begin and end index for range of BFAC.

NCOLR,NCOLT = Number of columns of data in receive and transmit patterns, respectively. 
NN1, NN2

NROWR, NROWT

NRX2R, NRX2T

$N X, N Y$

NXL1, NXL2

$N 1, N 2$

NIMAX, N2MAX

NIMIN, N2MIN

$N 10, N 20$

N11, N22

PHI, THETA, PSI PHIP, THETAP,

PSIP

PHIR, THETAR

PHIT, THETAT

PI

$R, T$

RADR, RADT

RCUT

$R G, T G$

SUM2

TKOKSQ

TSUM21

WAVLGTH

WORK

$X$
= Integer arrays of dimension (1) used in call to FFT subroutine FOURT and equal to $\mathrm{N} 1$ and $\mathrm{N} 2$, respectively.

= Number of rows of data in receive and transmit pattern, respectively.

$=$ NROWR and NROWT $\times 2$.

= Four times $N 1$ and $N 2$, respectively.

= Begin and end index for range of XLIM.

$=$ Integers equal to the number of $k_{x}$ and $k_{y}$ integration points, respectively.

= Integers determining maximum of the $x$ and $y$ range, respectively, over which the coupling quotient is plotted.

= Integers determining the minimum of the $x$ and $y$ range over which the coupling quotient is plotted.

= Intermediate integers used to define (N1MIN,N1MAX) and (N2MIN,N2MAX), respectively.

= Number of points in the $x$ and $y$ range, respectively, over which the coupling quotient is plotted.

= Eulerian angles of the left transmitting antenna as shown in figure 2 .

= Eulerian angles of the right, receiving antenna as shown in figure 3 .

= Spherical angles in the preferred coordinate system fixed in the right, receiving antenna, corresponding to the direction $k_{x} / k, k_{y} / k$

$\left(\emptyset_{p}, \theta_{p}\right.$ of eqs (13) and shown in figure 3$)$.

= Spherical angles in the preferred coordinate system fixed in the left, transmitting antenna, corresponding to the direction $k_{x} / k, k_{y} / k$

$\left(\varphi_{A}, \theta_{A}\right.$ of eqs (13) and shown in figure 2).

$=\pi=3.14159 \ldots$

= Complex array containing the spherical angle coordinates for the coordinate system fixed in the receiving and transmitting antenna, respectively.

= Radii of the smallest sphere circumscribing the right receiving and left transmitting antenna, from their respective origins.

= Maximum ordinate value for plots. If RCUT equals 0 , plot is self-scaled.

= Input reflection mismatch factor for receiving and transmitting antenna, respectively.

= Dummy summation variables used in the filling of the $A X$, AY matrices.

= Magnitude squared of the transverse part of the propagation vector.

= Summation variable used to compute the coupling quotient at $x 0=0$, $X_{0}=0$ by summing directly without the use of the FFT (as a check).

= Wavelength in meters.

= Complex array required only by FFT subroutine FOURT.

= Array containing the abscissa values for plots. 
XDUM

XK

XKLIM

XKMAX

XKMIN

XKXOK, XKYOK

XLIM

XMAX, XMIN

$X N X, X N Y, X N Z$

$X 0, Y 0, z 0$
= Dummy variable used in MINMAX when this subroutine is used with a one dimensional array.

$=2 \pi / \lambda$.

= Real variable which limits the range of $k_{x} / k$, and $k_{y} / k$ integration when its value is less than XKMAX.

= An upper bound (less than 1.0) on XKLIM; except for very close antennas XKLIM will usually be less than XKMAX anyway.

= Sum of the diameters of the two antennas divided by their separation distance; this variable is approximately proportional to the mutual angle subtended by the two antennas; XKLIM = XKMIN times XLIM when this product is less than XKMAX.

$=k_{x} / k$ and $k_{y} / k$, respectively.

= Intermediate variable used for adjusting XKLIM; making XLIM larger tests whether a wide enough spectrum has been included.

= Maximum and minimum abscissa values for plots.

= Variable used for incrementing $k_{x} / k, k_{y} / k$, and $\gamma / k$, respectively.

$=X, Y, Z$ coordinates of the origin of the right receiving antenna in the mutual coupling coordinate system of the left transmitting antenna; specifically $Z 0$ is the separation distance (d in eq (32)) between antennas.

File Names

INPUT, OUTPUT, TAPE 1, TAPE $3, \ldots$, TAPE 8

Subroutines Not Within FORTRAN Library (in alphabetical order)

ANGLGEN (Documented below)

FINDFF (Documented below)

FOURT (Standrd FFT subroutine with documentation within its own comment cards)

MINMAX (Documented below)

PLT120R (Page printer subroutine)

VECTGEN (Documented below) 
Functions Inline or within Computer Library (in alphabetical order)

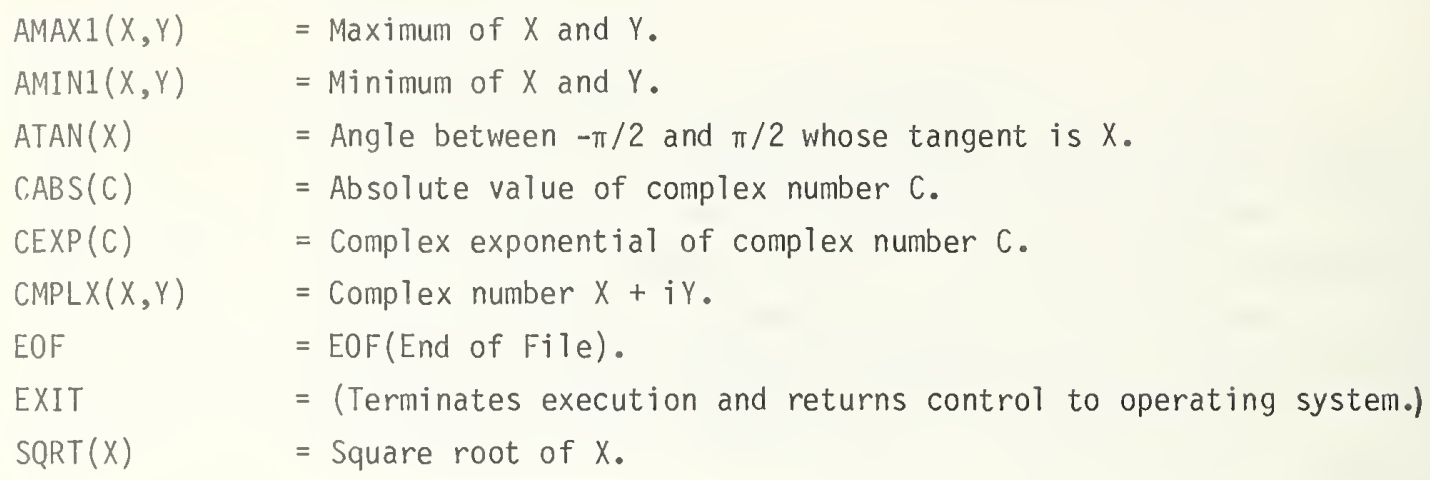

\section{List of Complex Quantities}

AX, AY, COEF, ETA, FDOTFP, FMM, FX, FXP, FXR, FXT, FY, FYP, FYR, FYT, FZ, FZP, FZR, FZT, R, SUM2, T, TSUM21, WORK, CEXP, CMPLX.

\section{COMMON BLOCKS:}

The labeled common in CUPLNF is described below with a list of routines in which it is used. The variables are defined in the symbol dictionary

COMMON /FAR/ N1, N2, NX, NY, OLX, DLY, XK, ISPECT

Routines using/FAR/: CUPLNF, FINDFF. 
PQDCRAM CIJPLNF (INPUT, ?UTPUT, TARFI, TAPF3, TAPE4, TAPF5,

UPLNF

1 TAPFS. TAPF7, TAQFE, TAQFGO=INPIJT)

CUPLNF

CUPLNF

5

GENEQAL INEDRMATITN $\triangle R O L T$ RROGRAM

THIS QRTGPAM COMDLITES THE CDUPLING OURTIFNT BETWEEN A

TPANSMITTING $\triangle N F F N N \triangle$ DN THE LFFT $\triangle N D$ A RECFIVING $\triangle N T E N N A$ ON THE

PIGHT IF $\triangle R B I T P A R Y$ PFLATIVF RRIFNTATION AND SEPARATION,

10

1 5

20 FRTM TUF GIVFN CDMPLEX FAR-FIELD PATTFRN TIF FACH ANTFNNA.

FHF CMHPLING DUTTIENT IS CMMPUTED $\triangle L O N G$ XO $\triangle N D$ YO RERPENDICULAR I INIFS MR CUTS.

$\triangle X, \triangle Y, A N O$ WIRK SHOIJLO BF OIMENSIDNED. GE. THE LARGEP DF (N1, N2). $\triangle C L C U T$ TND X SHOILD RE TIMENSIDNFD AT LFAST 2 GREATEP THAN THE L $\triangle R G F D$ DE (NI, N? ).

FYT, FYR, FXT, FZR, P, $\triangle N \cap T$ SHIULD BE DIMENSIONEN . GE. N?.

DATA SHRULD RF LARGF FNOUGH TO CINTAIN ALL DF THE INDUT FAR-FIELD CUPLNF EATA FIR FITHFR ANTENNA IE。 GF。2

RHI, THFTA, PSI $\triangle R E$ THE EUILFRIAN ANGLFS OF THF RFORIFNTFD TRANSMITTING $\triangle X F S$ WITH RESPFCT TA THE $\triangle X E S$ FIXED IN THE TDANSMITTING ANTENNA.

PHIR, THFTAR, PSIP $\triangle R F$ THF FULFPIAN $\triangle N G L F S$ CF THF RETRIFNTED

PFCFIVING $\triangle X E S$ WITH RESPECT TO THE $\triangle X F S$ FIXED IN THF

RFCFIVING ANTENNA.

(XO,YO,7O) $\triangle R E$ THF CCCRDINATES OF THE DPIGIN DF THE RECEIVING ANTFNNA IN THE RERRIFNTEO RFCTANGULAR SYSTFM TF THE TRANSMITTING $\triangle N T F N N A$.

THE RFTRIFNTED COPDINATF SYSTEMS DF FACH $\triangle N T F N N A$ ARE THE COMMDN M1JTUAL COIJPLING CROPDINATF SYSTFMS OF THE ANTENNAS.

THF COIRTINATE SYSTEM FIXFD IN FACH ANTENNA IS THE $¥ P P E F F R R E \#$ SYSTFM IN WHICH THE FAR-FIFLDS DF EACH ANTENNA ARE GIVFN.

ZO MUST QF SPFCIFTFD, BUT THF PANGE OF XO $\triangle N D$ YO ARE DFTERMINED

IMDLICTTLY RY THF PFOUIREMFNTS OF THE ALGQRITHM FDUPT.

QADT=QADIJS OF SMALLEST SRHERF WHICH CIRCUMSCRIBFS THF

TRANSMITTING ANTFNNA FRIBM ITS ORIGIN.

RADR=RARIUS OF SHALLEST SPHERE HHICH CIRCUMSCRIBES THF

PFCFIVING $\triangle N T F N N A$ FRCM ITS ORIGIN.

DI $\triangle M T=$ TWICE THF LARGEQ DF RADT OP WAVLGTH

DI $\triangle M D=$ TWTCF THE LARGER OF RADP OR WAVLGTH

BFAC $\triangle \Pi J U S T S$ THF INTFGRATION INCREMFNTS, $\triangle N O$ SHOULO BE

APPRTXIMATFLY 1 OR.? . MAKING BFAC LAPGFR TFSTS WHETHER

C CNVEPGFNCF HAS PFEN REACHED.

XLIM ATJISTS THE NONZERO-FILL PQRTICN TF THE INTEGRATION PANGE, $\triangle N D$ - SHOULO BE $\triangle R D D \cap X$. 1 MP ?. MAKING XLIM LARGFR TESTS WHETHER $\triangle$ WIDF FNDIIGH INTFGRATION RANGE HAS REFN INCLUDED. INCREASING XLIM ALSD TFCPEASFS THF INTEGPATTON INCPEMFNTS PROPDRTITNATELY I. PREVENT ALIASING.

$A 1, A 2,21, B 2$ DEFINE THE TDTAL (WITH ZERC-FILL) INTEGRATION RANGES

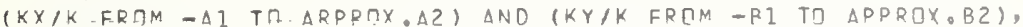
IN INCRFMFNTS DF $(\Delta 1+\Delta 2) / N 1$ QR $(R 1+R 2) / N 2 \triangle R R O X$. FQUAL TO OKDK. DKOK = WAVLGTH/(2*(DIAMT+DIAMP)*BFAC*XIIM)。

IF SQRT $(K X / K) * * 2+(K Y / K) * 2)$ IS GE . XKLIM THE SPECTRUM IS SET EOIJAL TO ZFRC. (APRRFCIALBLE ZERC FILLING IS AN GDTION DESIGNED.TO ALLOW FIN.ER INCREMFNTS DX AND DY AT WHICH THE C.CHPLING QUDTIFNT IS COMRUTED BY THF FFT.)

XKLIM MIJST RE EQUAL TO OR LESS THAN 1 BECAUSE

THF DRTGRAM NFGLECTS THF FVANESCFNT MODES. IN ORDER NUT TO GET CUPLNE TRO CLISE TH. THE I/GAMMA SINEULARITY, IT IS SAFER TH CHOOSF XKLIM NI LADGFP THAN XKMAX = $\triangle B \cap U T, Q$.

XLIM AIJUSTS XKLIM. IF AN ACCUPATE COUPLING QUOTIENT IS

R.EQUTRED ONLY FQQ SMALL VALUES OF XO $\triangle N \cap Y O /($ (DIAMT + DIAMP) * QFAC $*$ ILIM), XLIM NEED NOT BE MORE THAN 1 IOP 2. IF $\triangle C C U R A C Y$ IS DFSIRED FOR LARGER YO AND YO $\triangle S$ WELL, YLIM. SHOILD BF MADF CORPESDONBINGLY LARGFR. AS MENTIONEO ABQVE, MAKING XLIM LARGFQ TESTS WHFTHEQ A WIDE ENDUGH SPECTRUM HAS BFEN INCLUDFD.

THE XO $\triangle N \cap$ YO INCREMENTS ARE OX=WAVLGTH/ $(A 1+\Delta 2) \quad \triangle N D$ $D Y=W A V L G T H /(B I+R 2)$.

CUPLNF

CUPLNF

CUPLNF

CURLNF

CUPLNF

CUPLNF

CUPLNF

CUPLNF

CUPLNF

CURLNF

CUPLNF

C.UPLNF

CUPLNF

CUPLNF

CUPLNF

CUPLNE

CUPLNF

CUPLNF

CUPLNF

CUPLNF

CUR!NF

CUPLNF

CUPLNF

CUPLNF

CUPLNF

CUPLNF

CUPLNF

CUPLNF

CUPLNF

CUPLNF

CURLNF

CUPLNF

CUPLNF

CUPLNF

CUPLNF

CUPLNF

CUPLNF

CUPLNF

CUPLNF

CUPLNF

CUPLNF

CUPLNF

CUPLNF

CUPLNF

CUPLNF

CUPLNF

CURLNF

CUPLNF

CUPLNF

CUPLNF

CUPLNF

CUPLNF

CUPLNE

CUPLNF

CUPLNF

CUPLNF

CURLNF 
RLITTFN (WHFN XLIM\#RFAC IS GRFATER THAN QR FQUAL TO 1 ). CFF IS THF SRFFD OF LIGHT IN GIG $\triangle M E T F R S$ PER SFCOND. FMM IS THF MISM $\triangle$ TCH FACTOR FOP THE RECEIVING ANTENNA.

CUPLNF

CUPLNF

CUPLNE

CUPLNF

CUPLNE

SPERIFICATITN STATFMFNTS

CUPLNF

CUPLNE

CCMPLFX $\triangle X(1000), A Y(1000), T(1000), R(1000)$, WORK(1000)

CUPLNF

CTMPLEX FYT(I000), FYR $(1000)$, FZT(10C0), FZR 11000$)$

CחMPLFY FMM, COEF, GAMT, GAMR, GAML

CחMRIFX FXT, FXR

C. CMDLFX FX, FY, FY

COMFLFX FXR, FYP, F 7 D

COMPLFX FחDTFP

CIMRIFX TSIJM?], SUMZ

IIMFNSIMN NNI(1), NN2(1), HEAD(8), Iח(4)

DTMFNSIRN ACLCUT $(1010), x(1010)$, DATA $(8192)$

INTFGFR HE $\triangle D$

EQUIVALENCF (T,FZT), (R,FZR), (ACLCUT,FYT), (WORK,FYR)

FOUIJVLFNCF $(\triangle X, \cap \Delta T A(1)),(A Y, D \triangle T \Delta(2500)),(X, T)$

CRMMIN IFAR, NI, N?, NX, NY, DLX, DLY, XK, ISPFCT

DFFINITION $\triangle N D$ RFADING TF INPUT DATA

In $A N \cap 4 F A \cap$ ARF $A L P H A N U M F R I S$ IDFNTIFIERS

ID IS PROBR AMMERS NAMF ANID PHONE FXTENSION

HF $\triangle D$ IS THF IDENTIFIFR FOR THE CASF UNDER STUDY

FRFQ = FDEOUFNCY OF DRFRATION IN GHZ

= SFPARATION RFTWFFN ANTFNNA REFERENCF POINTS ISFE COMMENTS $\triangle D \cap$ D I

OLXT = X-INCQFMENT WHICH CORRESPONDS TO KX INCREMENT XMIT

DLYT. = Y-INCREMENT WHICH CORRESPONDS TO KY INCREMENT XMIT

NLXR = X-INCREMENT WHTCH CORRESPQNDS TO KX INCREMENT RECV

ILYR = Y-INCREMFNT WHICH CORPESPONDS TO KY INCREMENT RECV

FTOFR = RATIO OF CHARACTFRISTIC ADMITTANCF OF TRANSMITTING $\triangle N T E N N A$ FFED MIIE TO CHARACTERISTIC ADMITTANCE OF THE RECFIVING, ANTFNNA FFED MDOE

ISRFCT = SRECTRLIM FLAG - SET FQUAL TD 1 IF INPUT DATA IS SPECTRUM RATHFR THAN FAR FIFLI

RCHT = MAXTMIJM TRDINATE VALIIF FMR PLOTS, IF RCUT.FO. O, PLOT I S SFLF-SCALED

NBFI, NRF? = RFGIN $\triangle N D$ END INDFX FDR RANGE DF BFAC

$N \times L], N Y L ?=R E G I N \triangle N \cap E N \cap$ INDFX FOR RANGE TF XLIM

GAINT = GAIN TF XMIT $\triangle N T F N N A$ IN DB.

FFTMX = MAGNITUDF OF FAR-FTELO PATTERM (UNNORMALIZFD) AT THFTA $=0$, XMIT

RADT = RADILS OF TRANSMIT $\triangle N T E N N A$ (SFE COMMENTS $\triangle B O V E$ )

PHI, THFTA, PSI = EULER ANGLES IN DEGREFS FIR XMIT ANTENNA ISEE CTMMENTS $\triangle B \cap V E$,

NPחWT = NIJMRER OF ROWS OF DATA IN TRANSMIT PATTERN

NCCLT = NIJMBFR DF COLUMNS DF DATA IN TRANSMIT PATTERN

ID $\triangle H R T=$ FILE INENTIFIER FIR XMTT DATA

CUPLNF

CIJPLNE

CUPLNF

CUPLNE

CUPLNF

CUPLNF

CUPLNE

CUPLNF

CUPLNF

CUPLNF

CUPLNF

CUPLNF

CUPLNF

CUPLNF

CUPLNF

CUPLNF

CUPLNF

CUPLNF

CUPLNF

CUPLNF

CUPLNF

CURLNF

CUPLNF

C, UPLNE

CUPLNF

CUPLNF

CUPLNF

CUPLNF

CURLNF

CUPLNF

CUPLNE

CUPLNF

CUPLNF

CUPLNF

CUPLNF

CUPLNE

CUPLNF

CURLNF

CUPLNF

CUPLNF

CUPLNF

CUPLNF

CUPLNF

CUPLNF

CUPLNF

CUPLNF

CUPLNF

CUPLNF

CUPLNF

CUPLNF

CUPLNF

CUPLNF

CUPLNF

- GAINR = GAIN DF RECV ANTENNA IN DR.

FFDMX $=M \triangle G N I T U D F$ DF FAR-FIFLD PATTFRN (UNNCRMALIZFD) $\triangle T$ THFTA $=0$, RECV

D DRR = PANIUS DF RECFIVE ANTENNA (SFE COMMFNTS AROVE)

CUPLNE COMMFNTS ABOVE)

NRTWR = NUMREQ OF ROWS OF DATA IN RECEIVE PATTFRN

NC $\cap L Q=$ N!JMAFR IF COLUMNS DF DATA IN RECFIVF RATTERN

CUPLNF

C, AMT = RFFLECTIDN CCEFFICIENT OF TRANSMITTING ANTENNA

$G A M R=$ REFLFCTION COEFFICIENT TF RECFIVING $\triangle N T E N N A$

$5 \triangle M L=$ REFLFCTION CCEFEICIENT OF PECEIVING LOAD

CUPLNF

CUPLNF

CUPLNE

CUPLNE

CUPLNE

CUPLNE

CUPLNF

RRINT 5205

RFA $5000,(I \cap(I), I=1,4)$

CUPLNE 
C

21 DDINT 5001 , HEAD
CUPLNF $\quad 156$

CUPLNF 157

CUPLNF $\quad 158$

CUPLNF 159

TUPLNF 160

CUPLNF 161

CUPLNF 162

CUPLNF 163

CUPLNF $\quad 164$

CUPLNF 165

CUPLNF $\quad 166$

CUPLNF 167

CUPLNF 168

CUPLNF 169

CUPLNF 170

CUPLNF $\quad 171$

CUPLNF $\quad 172$

CUPLNF $\quad 173$

CUPLNF $\quad 174$

CUPLNF $\quad 175$

CUPLNF $\quad 176$

CUPLNF $\quad 177$

CUPLNF $\quad 178$

CUPLNF $\quad 179$

CUPLNF 180

CUPLNF $\quad 181$

CUPLNF 182

CUPLNF 183

CUPLNF 184

CUPLNF 185

CUPLNF 186

CUPLNF 187

CIIPLNF 188

CUPLNF 189

CUPLNF 190

CUPLNF 191

CUPLNF 192

CUPLNF 193

CUPLNF 194

CUPLNF 195

CUPLNF $\quad 196$

CUPLNF 197

CUPLNF 190

CUPLNF 199

CUPLNF 200

CUDLNF 201

CUPLNF 202

CUPLNF 203

CUPLNF 204

CUPLNF 205

CUPLNF 206

CIJPLNF 207

CUPLNF 208

CUPLNF 200

CUPLNF 210

CUPLNF 211

CUPLNF $21 ?$

CUPLNF 213

CUPLNF 214

CUPLNF 215

CUPLNF 213

CUPLNF 217

CUPLNF 218

CUPLNF 219

CUPLNF 220

CUPLNF

CUPLNF 222

CUPLNF 223

CUPLNF 224

CUOLNF 225

CUPLNF 226

CUPLNF 227

CUPLNF 228

CUPLNF $\quad 229$

CIIPLNF

CUPLNF 231 


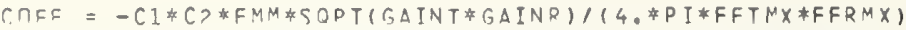

C1)PLNF

$R G=S O D T(1,-C A B S(G A M D) \neq 2)$

$T F_{3}=S 2 R T(1 .-C A R S(G A M T) * * 2$

C RFF $=$ COFF*RG*TG\#SODT(FTDFR)

T SUM? $1=(0.0 .1$

$N Y=4 * N$ ?

$N X=4 * N ?$

or in $\mathrm{J}\rangle=1, \mathrm{NY}$

$\triangle \times(J ?)=(0,0,0)$

$\triangle Y(J)=(0,0,0)$

10 CDNTINIF

PQINT 15, N1, N2

Dח $200 \mathrm{~J} 1=1, \mathrm{~N} 1$

$X N X=C 1 \neq(J 1-1 \cdot)$

$X K Y \cap K=X N X-A 1$

กก $100 \mathrm{~N} 2=1, \mathrm{~N} 2$

$X N Y=C ? *(\mathrm{~J} 2-1.1$

$X K Y \cap K=X N Y-B 1$

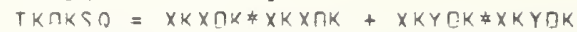

in $12 \mathrm{~J}=1$, NY

$\Delta Y(J \geq)=-\Delta Y(J 2) *(-1) * J$ ?

1? CONTINIIF

$N N 1(1)=N X$

CUPLNF

CURINF

CUPLNF

CUPLNF

CUPLNF

CUPLNF

CUPLNF

CUPLNF

CUPLNF

CUPLNF

CUPLNF

CUPLNF

CUPLNF

CURLNF

CUPLNF

CUPLNF 


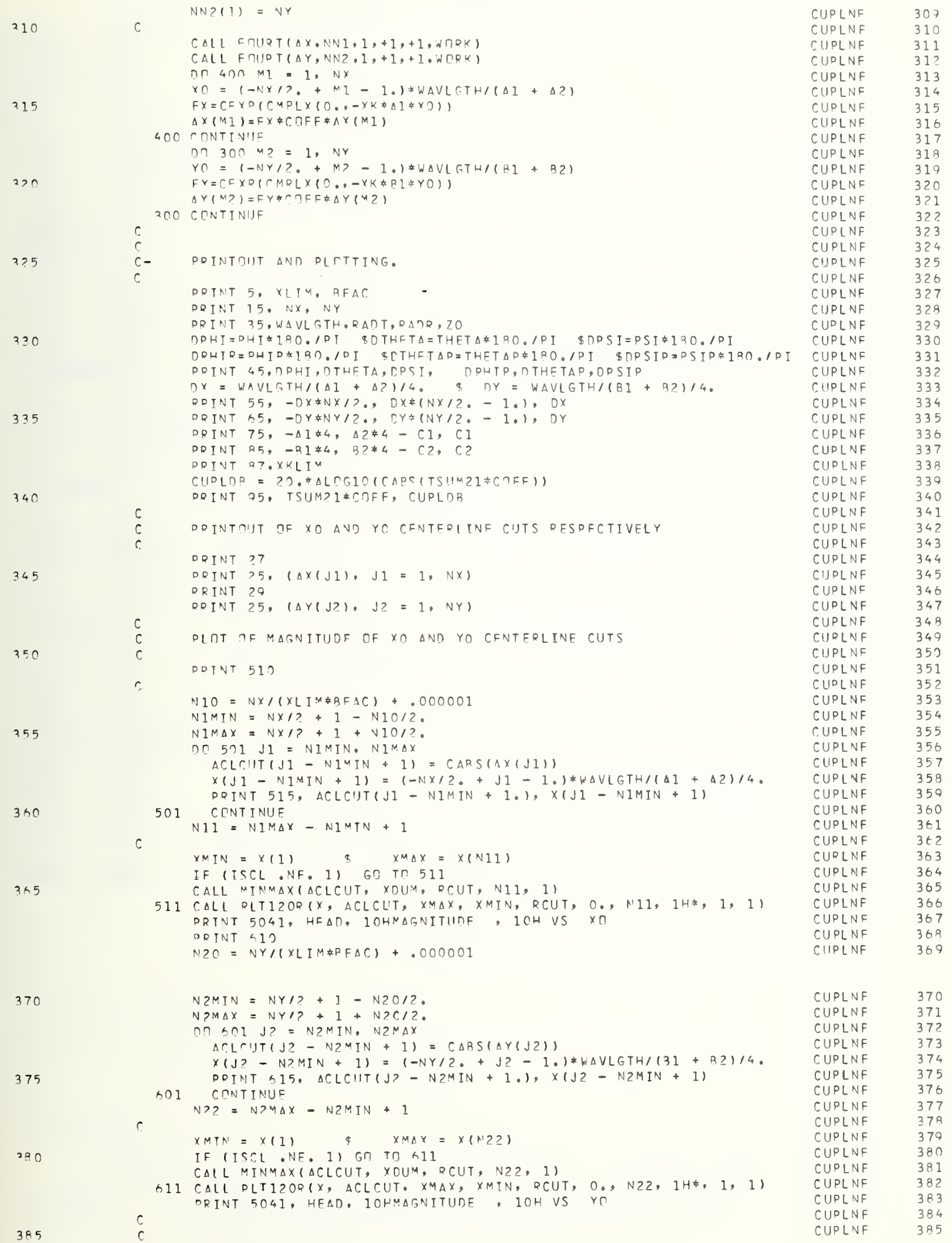




\section{B.1.2 SUBROUTINE ANGLGEN \\ (PKXOXK, PKYOXK, PHI, THETA, PSI, PHIP, THETAP, PSIP, PHIT, THETAT, PHIR, THETAR)}

\section{PURPOSE:}

To compute in the coordinate system fixed in each antenna, the far-field angles corresponding to a given direction of the propagation vector in the mutually common xyz coordinate system (see fig. 1).

METHOD:

Evaluate eqs (13a) and (13b) of the main text.

ARGUMENTS:

Input Parameters (in order of appearance)

PKXOXK,PKYOXK $=x$ and $y$ components of the normalized propagation vector $\left(k_{x} / k, k_{y} / k\right.$ of eqs (13)).

PHI, THETA,PSI = Eulerian angles of the antenna on the left as drawn in figure $2(\emptyset, \theta$, of eqs (13)).

PHIP, THETAP,

PSIP = Eulerian Angles of the antenna on the right as drawn in figure 3 $\left(\emptyset^{1}, \theta^{1}, \psi^{1}\right.$ of eq $\left.(13)\right)$.

Output Parameters (in order of appearance)

PHIT, THETAT = Spherical angles in the coordinate system fixed in the left transmitting antenna, corresponding to the direction $k_{x} / k, k_{y} / k\left(\emptyset_{A}, \theta_{A}\right.$ of eqs (13) and shown in figure 2).

PHIR, THETAR = Spherical angles in the coordinate system fixed in the right, receiving antenna, corresponding to the direction $k_{x} / k, k_{y} / k\left(\emptyset_{p}, \theta_{p}\right.$ of eqs (13) and shown in figure 3 ).

SYMBOL DICTIONARY:

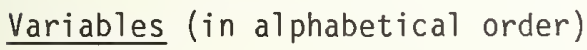

CSPH, CSPHP, CSPS, CSPSP,

CSTH,CSTHP = Cosine of PHI, PHIP, PSI, PSIP, THETA, and THETAP, respectively.

GAMOXK $\quad$ Normalized $z$-component of propagation vector $(\gamma / k)$.

PI $\quad=\pi=3.14159 \ldots$ 


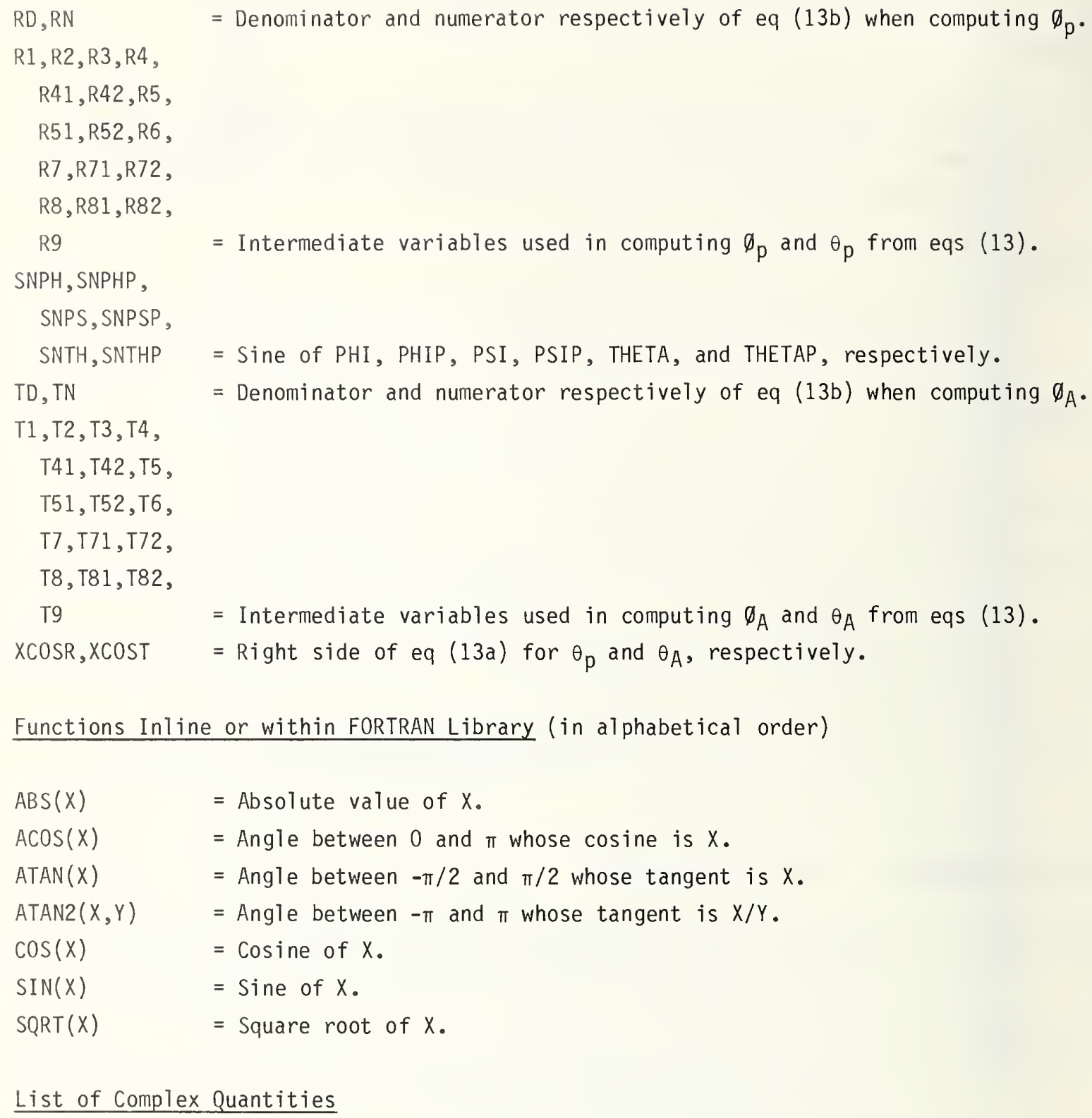

(None) 
$R 42=$ SNPHP $\$$ SNPS

$24=(241-P 42) * P K \times] \times K$

T5I = CSOH\#CSTH\#SNPS

$T=?=S N P H+C S R S$

$T 5=(T 51+T 5 ?) \neq P K Y \cap \times K$ $\triangle N G L G F N$

$\triangle N E L G E N$

$\triangle N E L G E N$

$\triangle N G L G E N$

$\triangle N G L G F N$

ANGLGEN

$\triangle N G L G E N$

$\triangle N G L G E N$

$\triangle N G L G E N$

$\triangle N G L G E N$

$\triangle N G L G E N$

$\triangle N G L G E N$

$\triangle N G L G F N$

$\triangle N G L G F N$

$\triangle N G L G E N$

$\triangle N G L G E N$

$\triangle N G L G E N$

$\triangle N G L G F N$

$\triangle N G L G E N$

$\triangle N G L G E N$

$\triangle N G L G E N$

$\triangle N G L G E N$

$\triangle N G L G E N$

$\triangle N G L G E N$

$\triangle N G L G F N$

$\triangle N G L G E N$

$\triangle N G L G E N$

$\triangle N G L G E N$

$\triangle N E L G E N$

$\triangle N E L G F N$

$\triangle N E L G E N$

$\triangle N G L G E N$

$\triangle N G L G E N$

$\triangle N G L G E N$

ANGLGFN

$\triangle N G L G E N$

$\triangle N G L G F N$

$\triangle N G L G E N$

$\triangle N G L G E N$

$\triangle N G L G E N$

$\triangle N G L G E N$

$\triangle N G L G F N$

$\triangle N E L G F N$

$\triangle N G L G E N$

$\triangle N G L G E N$

$\triangle N G L G E N$

$\triangle N G L G F N$

$\triangle N G L G F N$

ANGLGEN

$\triangle N G L G E N$

$\triangle N G L G E N$

$\triangle N \in L G E N$

ANGLGEN

$\triangle N G L G F N$

$\triangle N G L G E N$

$\triangle N G L G E N$

$\triangle N G L$ GEN

$\triangle N G L G E N$

$\triangle N G L G E N$

$\triangle N G L G E N$

$\triangle N G L G E N$

$\triangle N G L G E N$

$\triangle N G L G F N$

$\triangle N G L G E N$

$\triangle N G L G F N$

$\triangle N G L G E N$

ANGLGEN

$\triangle N E L G E N$

$\triangle N G L G E N$

$\triangle N G L G E N$

ANGL GEN

$\triangle N G L G E N$

$\triangle N G L G E N$

$\triangle N G L G E N$

$\triangle N G L G E N$

$\triangle N G L G E N$

$\triangle N G L G E N$
$T 7 ?=$ CSPH+CNPS

$T 7=(T 7 I+T 7 ?) * P K \times \cap \times K$

D71 = CNPHD\#CSTHD*CSPSR 
$T$ TPI $=$ NSPHWCSPS

$T R P=$ CNDH*CSTH\#SNPS

$\triangle N G L G E N$

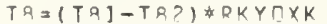

$\triangle N E L G F N$

$\triangle N G L G F N$

$\triangle N G L G F N$

$\triangle N G L G F N$

$R R 1=C S P H Q$ \& $C S P S P$

RP? = SNPHP*C.STHR *SNDSP

$\triangle N G L G F N$

$\triangle N G L G E N$

$\triangle N G L G F N$

$\triangle N G L G E N$

$\triangle N E L G F N$

$T C=$ SNPH\#SNTH*GAMOXK

an

$R G=S V R H D * S N T H P \# G A M \Gamma X K$

$\triangle N G L G E N$

ANGLGEN

RN $=R 7-R R+R Q$

C.HANGF RF RANGF DF PHIT FRחM(-RI, PI) TR $(0.2 P I)$.

$\triangle N G L G F N$

$\triangle N G L G F N$

$I F((\Delta Q S(T N)+\Delta Q S(T \cap)), F O .0$.$) EO TO 10$

ANGLGFN

$R H I T=A T A N)(T N, T \cap)$

IF (PHIT.LT.O.) PHIT $=2$. *PI + PHIT

$A N G I G F N$

$\triangle N G L G F N$

GQ Tก? ?

$1 ก$ ก

1) CONTJNIF

QHTT $=$ ?

30 CMNTINHIF

CHANEF CF DANGE CF DHJP FRMM(-PI, DI) TD $(0.2 P I)$.

$\triangle N G L G F N$

$\triangle N G L G F N$

$\triangle N E L G F N$

$\triangle N G L G F N$

$\triangle N G L F F N$

$\triangle N E L G F N$ PHIR = $\triangle T A N ?(D N, R D)$

ANGLGFN

$\triangle N E L G F N$

IF (PHID.LT.O.) PHIR=? \#DI + DHIR

$\triangle N G L G F N$

GR Tח 40

$\triangle N G L G E V$

36 CONTINIJ

$$
\text { DHIR = O . }
$$

$\triangle N G L G F N$

$\triangle N G L G F N$

RFYURN

$\triangle N G L G F N$

FND

$A N E L$ GFN 


\section{B.1.3 SUBROUTINE FINDFF (IDAYHR, LUIN, LUA, LUOZ, LUOE, DATA, NRX2, NCOL, FFY, FFZ, STOR)}

PURPOSE:

To read from an input file, spectrum or far-field data whose coordinates are $k_{x}$ and $k_{y}$ referred to the antenna's preferred coordinate system and from this produces a file containing far fields whose coordinates are specified by the angles specified on a second input file.

\section{ARGUMENTS:}

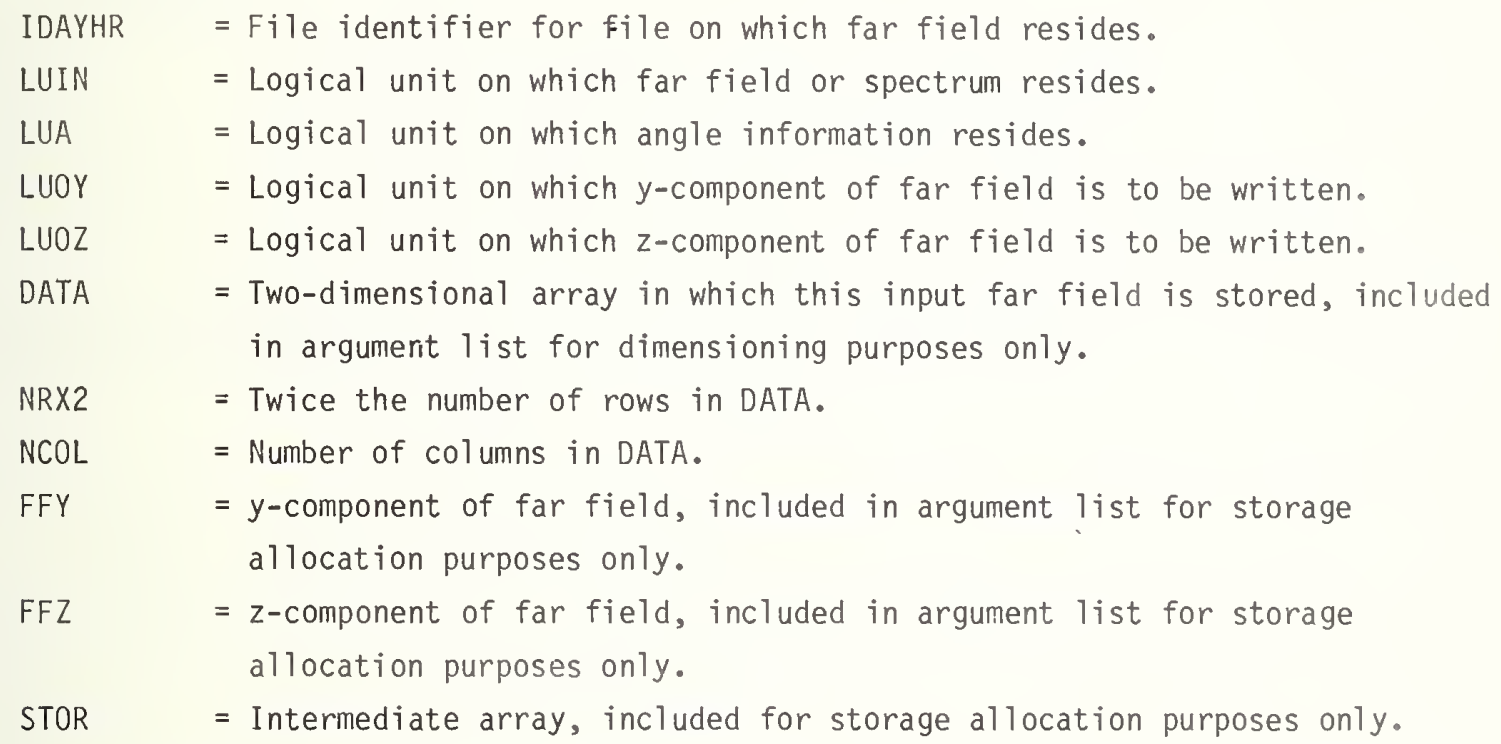

\section{METHODS:}

The subroutine reads the first record of the file containing the far-field or spectrum data from unit LUIN and compares the eighth word of the record with IDAYHR in order to make sure the proper data file is used. If LUIN contains the incorrect file, execution terminates. After correct file verification, the entire file is read in and stored in array DATA. Because the input data exist in polar form, a conversion to rectangular form is also performed in the operation of filling DATA.

A11 data transfers use FORTRAN unformatted READ and WRITE operations.

The desired far-field angles are assumed to be stored on unit LUA. These are read one record at a time into complex vector FFY with the real part containing the $\emptyset$-coordinate and the imaginary part, the $\theta$-coordinate. For each element of the vector FFY, the angles stored are used to locate the nearest far-field point in the array DATA. The z-component is then calculated and stored in FFZ. When all angles in FFY have been changed to the corresponding far-field values, the vectors FFY and FFZ are written out as a record on units LUOY and LUOZ, respectively. 
The correct point in the far-field array is found by the following procedure. calculate the reference index for the $x$ and $y$ directions by

$$
\begin{aligned}
& I_{c}=\text { integer part of } \frac{k_{y}}{\Delta k_{x}} \\
& I_{r}=\text { integer part of } \frac{k_{y}}{\Delta k_{y}}
\end{aligned}
$$

where

$$
\begin{aligned}
& k_{x}=k \sin \theta \cos \emptyset \\
& k_{y}=k \sin \theta \sin \emptyset
\end{aligned}
$$

and $\Delta K_{x}$ and $\Delta k_{y}$ are the far-field $k_{x}$ and $k_{y}$ increments. These increments are given by

$$
\begin{aligned}
\Delta k_{x} & =\frac{2 \pi}{N_{x} \delta} \\
\Delta k_{y} & =\frac{2 \pi}{N_{y} \delta_{y}}
\end{aligned}
$$

where $N_{x}, N_{y}$ are the number of $x$ or $y$ points and $\delta_{x}, \delta_{y}$ are the $x$ or $y$ near-field spacing.

The far-field increments are given in terms of near-field spacings because it is assumed that the far field is obtained either by a near-field scan or the PO model program POMODL (see appendix A), which calculates its far-field array based on a desired near-field spacing.

The row and column indices $I_{r}$ and $I_{C}$ specify "lower left-hand corner" of the square in $\left(k_{x}, k_{y}\right)$ space which contains the point specified by the angles $\theta$ and $\emptyset$. The fractional part of

$$
\frac{k_{x}}{\Delta k_{x}} \text { or } \frac{k_{x}}{\Delta k_{y}}
$$

is used to determine which corner of the square lies closest to $\theta$ and $\emptyset$. 
The z-component is found from the relationship

$$
E_{z}=E_{y} \tan \theta \sin \emptyset
$$

Because the far-field array DATA may not contain values for all angles, a test is performed to determine if DATA does, in fact, contain a far-field value at the requested $\theta$ and $\phi$. If it does not, the $y$ - and $z$-components are set to zero.

\section{SYMBOL DICTIONARY:}

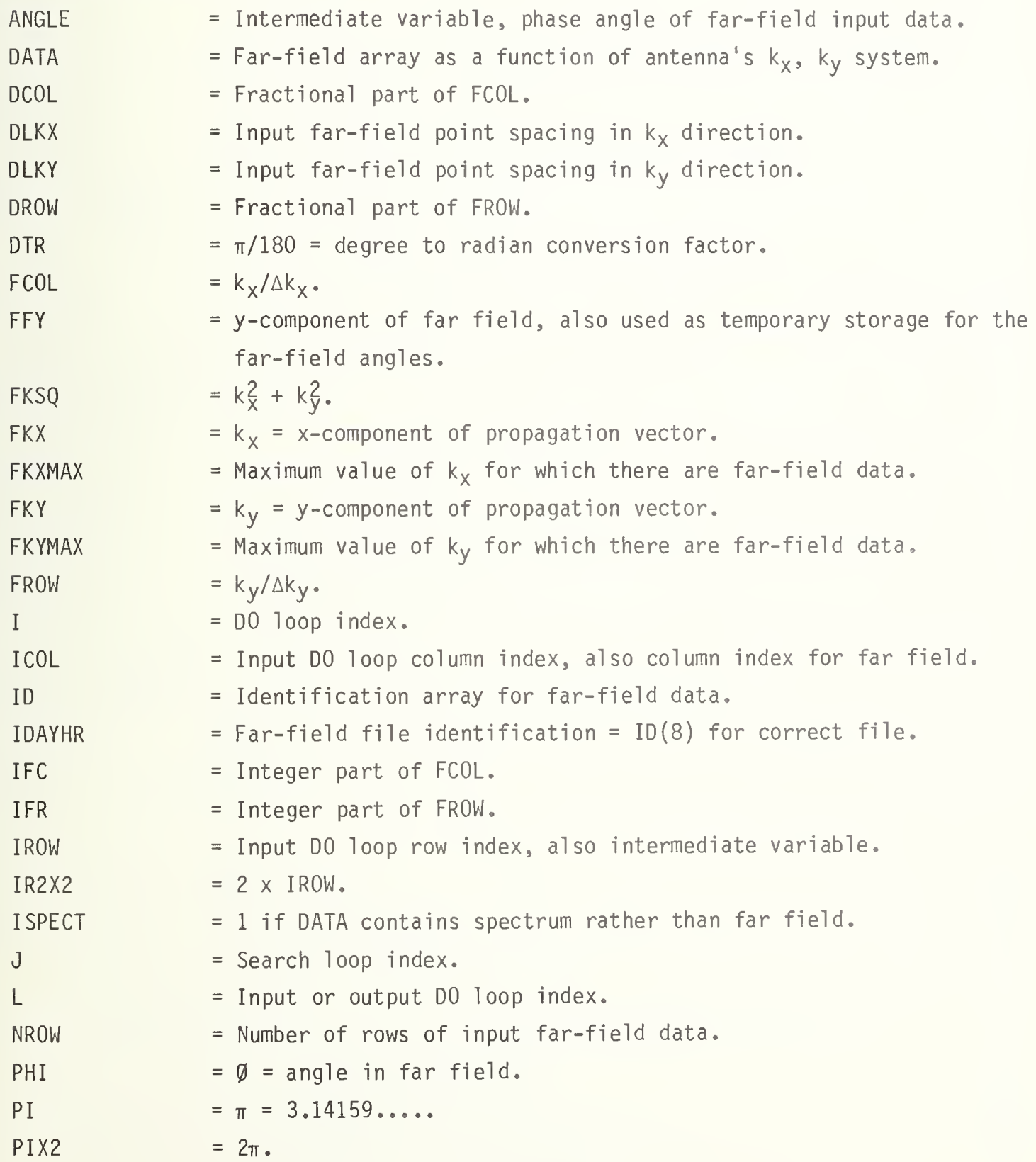


TAMP

THETA

$X N Z$
= Intermediate variable, amplitude of input far-field data.

$=\theta=$ angle in far field.

$=\cos \theta$. 
C- THIS SUIRRIIITINE READS FAF-FIELO TRR SPECTRUM DATA FROM LUTN AND

C- STIRRFS IT IN ARRAY DATA. ANGLFS CORRESOPNOING TC FAR-FIFLD

C- OIPFCTITNS IN THF ANTFNNAS CIOPOINATE SYSTEM ARF READ IN FRDM LUA.

C- DATA IS SEAPCHFI FIR THF CLIMSEST RIINT $\triangle N D$ THF Y-COMRONENT DF THE

C- FIEIT $\triangle T$ THE GIVEN ANGLE IS USFD TD CCMPUTE THE Z-COMPONENT. THFSF FIFLI CIMPONFNTS APF WRITTFN DN LUCY ANO LUOZ.

C. MMPLFX FFY $(1), F F Z(1)$

CCMMIN /FAR/ N1, NZ, AX, NY, DLX, DLY, XK, ISPFCT

DIMENSTMN DATA(NRX2, NCOL), STOR(1). ID(10)

$c-$

\section{c- MISCFLLANFOUS}

\section{RRINT 1020, LIIIN, LUA, LIITY, LUOZ, NRXZ, NCCL}

1020 FIRMAT (6I)

$T S F=0$

$P I=4 . * \Delta T A N(1$,

$P T Y 2=2 . * R T$

$D T R=D T / L B O$.

NRPW = NRX?

$n I K Y=R I X \supset / N R \cap W / \cap L Y$

$D L K X=D T X ? / N C T L / D L X$

$F K X M \triangle X=O L K X(N C O L-1) / 8$

FKYMAX $=D L K Y *(N R M W-1) / ?$

PRTNT 10OO, OLKX, DLKY, FKXMAX, FKYMAX, XK

1000 FחRMAT $(1 \times, 5620.5)$

C- FINM CROPFCT FAP-FIFLO FILF IN L RGICAL UNIT IIIIN.

6

120 PFAM(LHIN) (TOU I , T = 1,10$)$

PRINT 1510, ID

IF $(I 0(B), F Q$. ID $\triangle Y H R) \quad F \cap T D 130$

IF (FAF(LIJIN)) 125, 130

125 PRTNT 1530

CALL FXIT

40

130 C TNTINHF

$\mathrm{C}$

C- DFAD FAR-FIFLD INTO $\triangle R R A Y$ DATA.

C. -

Oก 14C. IC, חL $=1$, NCOL

RF $\triangle D(1 \| I N)(S T O P(I), I=1, N R \times ?)$

45

Dก 150 IRחW $=1$, NPCW

$I D Y ?=I P T W * 2$

$T A M P=S T C R(I R \times 2-1)$

$\triangle N C, L=S T O R(T O \times 2) * N T D$

$\triangle A T A(I P X)-1, I C[L)=T A M D C M S(\triangle N G L F)$

50

$\cap A T A(I R X), I C I L)=T \Delta M P$ SIN $(\Delta N G L F)$

150

CONT INLIF

140 CONTINIF

55

c-

- RFPLACF YALIJES TTF $\triangle N G L F S$ WITH CORPFSPONCING FAR-FIFLI.

c-

SO

WIT ND LUA

RFA (LUTN)

IF (ETF(LHIN)) 500,600

SCO CONTINUIJF

PACKSOACF LIIIN

-CO CONTINIJ

OD $200 T=1, N I$

RFAO(111A) (FFY (L), L = 1, N2)

Dก $300 \mathrm{~J}=1, \mathrm{~N}_{2}$

DHI $=$ PEAL (FFY (J))

FHETA $=\triangle I M A E(F F Y(J))$

TF (THETA LT. D.) GO TO 310

C- FIND THF TNOTCFS FIR THF DRPAY TATA WHICH CTRRFCDOND TI THF

$7 n$

$r-$ STOROINATES CLOSFST TO THF DFSIRFD THFTA ANO RHI VALUES.

$F K X=X K * S I N(T H E T A) * C T S(P H I)$

$F K Y=X K * S T N(T H F T A) * S I N(P H T)$

$F K S O=F K X * F K X+F K Y * F K Y$

75

TF $(\triangle A S(F K X)$, FF. FKXMAX) CO TC 310

IF $(\Delta R S(F K Y)$. GF. FKYMAX) G] TГ 210 


\section{B.1.4 SUBROUTINE VECTGEN ( $F O X, F O Y, F O Z, P H, T H E T, P S, F X, F Y, F Z$ )}

PURPOSE

Given the components (FOX,FOY,FOZ) of a complex vector in a right-hand rectangular coordinate system, find the transformed components ( $F X, F Y, F Z$ ) of that vector in a second coordinate system formed by rotation of the first through the Eulerian angles $(\mathrm{PH}, \mathrm{THET}, \mathrm{PS})$.

METHOD:

Use the transformation given by eq (18) of the main text.

\section{ARGUMENTS:}

Input Parameters (in order of appearance)

FOX, FOY,

FOZ $=x, y, z$ rectangular components of the given complex vector in the unrotated coordinate system.

PH, THET,PS = Eulerian angles of the rotated coordinate system.

Output Parameters (in order of appearance)

$F X, F Y, F Z$ = Transformed $x, y, z$ rectangular components of the given complex vector in the rotated coordinate system.

SYMBOL DICTIONARY:

$\underline{\text { Variables }}$

A1 1, A12,

$\mathrm{A} 13, \mathrm{~A} 21$,

$A 22, A 23$,

$A 31, A 32$,

A33 = The nine elements of the $3 \times 3$ matrix on the right side of eq (18).

CSPH, CSPS,

CSTH = Cosine of PH, PS, and THET, respectively.

SNPH, SNPS,

SNTH = Sine of PH, PS, and THET, respectively. 
Functions Inline within FORTRAN Library

$$
\begin{aligned}
& \cos (x)=\text { cosine of } x . \\
& \sin (x)=\text { Sine of } x .
\end{aligned}
$$

\section{List of Complex Quantities}

$$
F X, F Y, F Z \text {, FOX, FOY FOZ }
$$


1

5

10

15

20

25

30

35

SUIPROIJTINE VFCTGEN(FCX, FOY, FOZ, PH, THET, DS, FX, FY, FZ)

VEC TGEN IF THE COMPDNENTS (FOX.FOY,FOZ) DF A CCMPLEX VECTCR ARE GIVEN IN VECTGEN A EIGHT-HANCFD RECTANGILAD CRORDINATE SYSTEM, ANC A SECDND

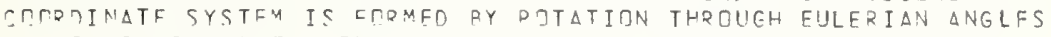
(DH,THET, PS), THFN (FX, FY,FZ) ARF THF CMMPONENTS IF THAT VECTDR IN THIC SFCINIO DOTATEN SYSTEM.

COMDLEX FOY, FOY, FOZ

CDMPLFX FX, FY,FZ

COMDUTATIMN OF THE NINF ELEMENTS OF THE DQTATIONAL TPANSFTRM $\triangle T I C N$ MATRIX.

$\angle C O H=C O S(D H)$

SNPH $=S I N(P H)$

$C S D S=\operatorname{CHS}(D S)$

SNDS = SIN $(D S)$

$C S T H=C \cap S(T H F T)$

SNTH $=$ SIN $(T H E T)$

C

$A 11=C S P H * C S T H * C S P S-S N P H * S N P S$

$\Delta 12=$ SNDH\#CSTHACSPS + CSOHASNPS

$\Delta 13=-S N T H * C S P S$

$\triangle 21=-(C S P H * C S T H * S N D S+$ SNPH*CSPS $)$

$\Delta 22=-S N D H * C S T H * S N D S+C S P H * C S P S$

$\Delta>2=S N T H * S N D S$

$\Delta 31=C S D H \# S V T H$

$\Delta 3 ?=S N P H \neq S N T H$

$\Delta 22=C S T H$

C

$F X=\Delta 11 * F 0 X+\Delta 12 * F 0 Y+\Delta 13 * F 07$

$F Y=A 21+F O X+\Delta 2 ?+F O Y+A ? 3+F O$ ?

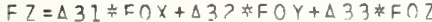

RETIIRN

FND

VECTGEN

VECTGEN

VECTGEN

VECTGEN

VECTGEN

VECTGEN

VECTGEN

VECTGEN

VECTGEN

VECTGEN

VECTGEN

VECTGEN

VECTGEN

VECTGEN

VECTGEN

VECTGEN

VECTGEN

VECTGEN

VFCTGFN

VECTGEN

VECTGEN

VECTGEN

VECTGEN

VECTGEN

VECTGEN

VECTGEN

VECTGEN

VECTGEN

VECTGEN

VECTGEN

VECTGEN

VECTGEN

VECTGEN

VECTGEN 


\section{B.1.5 SUBROUTINE MINMAX(Z,ZMIN,ZMAX, LEX, LEY)}

PURPOSE:

To determine the maximum and minimum values stored in the array $Z$.

ARGUMENTS:

$Z$ is a two-dimensional array which is to be searched for its maximum and minimum values.

ZMIN contains the minimum value in the array $Z$ on exit.

ZMAX contains the maximum value in the array $Z$ on exit.

LEX is the number of rows in $Z$.

LEY is the number of columns in $Z$.

METHODS:

Array $Z$ has dimensions (LEX,LEY). Initial7y ZMIN and ZMAX are set equal to $Z(1,1)$. Each value of $Z$ is tested to determine if it is less than ZMIN or greater than ZMAX. If either condition is satisfied, ZMIN or ZMAX is appropriately changed.

\section{SYMBOL DICTIONARY:}

= Row DO loop index.

J

= Column DO loop index.

TZ

= Temporary variable, $Z(I, J)$. 
1

5

1 ก

SIRQר!T TNF NTNMAX(7, TMYN, 7MAX, LFX, LFY

OINEVCITN T(LEX, LEY)

ZM $T:=7(1,1)$ \& $7 M A Y=7(1,1)$

ne. $120 \mathrm{~T}=1, \mathrm{~L}$ CX

$n \cap 1 ? n .1=1, \mathrm{LFY}$

$T 7=7(T, 1)$

IF (T? IIT. ZMIN) ZMTN $=T Z$

TF (TT.GT. TMAX) TMAX $=T$ T

is crittinir

$5 \mathrm{~T} \operatorname{Tin} \mathrm{V}$

FNI

MIA.M $A X$

MINMAX

$M I N M A X$

$M I N A X$

NINMAX

MINMAX

MINM $\triangle X$

MINMAX

YINM $\Delta X$

NINMAX

MINMAX

10

11 
Illustrated below is a typical input card deck for program CUPLNF. Far-field data for the two antennas were generated using POMODL. The output obtained for one of these runs is illustrated in Appendix A.2. The output produced by CUPLNF is reproduced on the following pages.

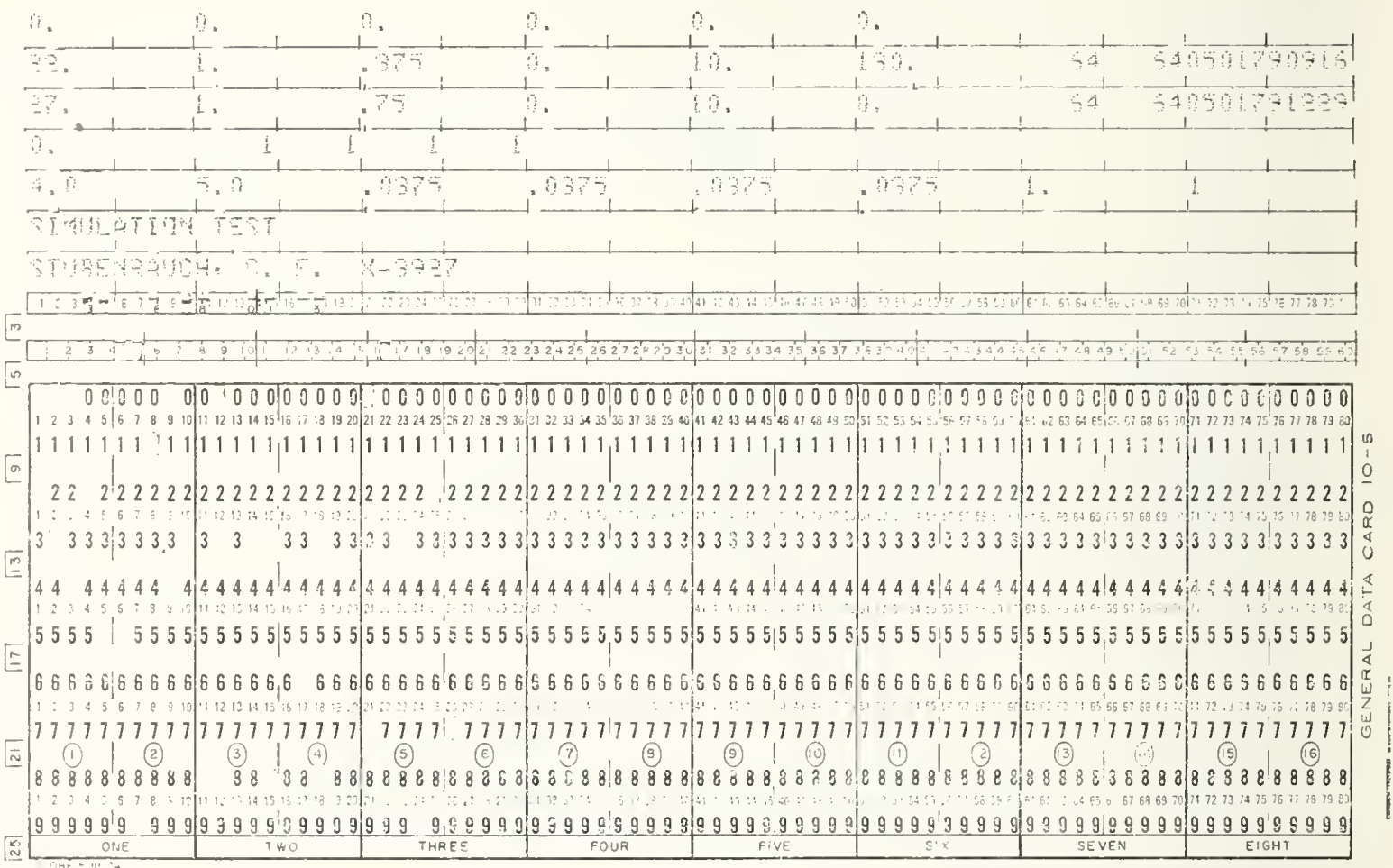




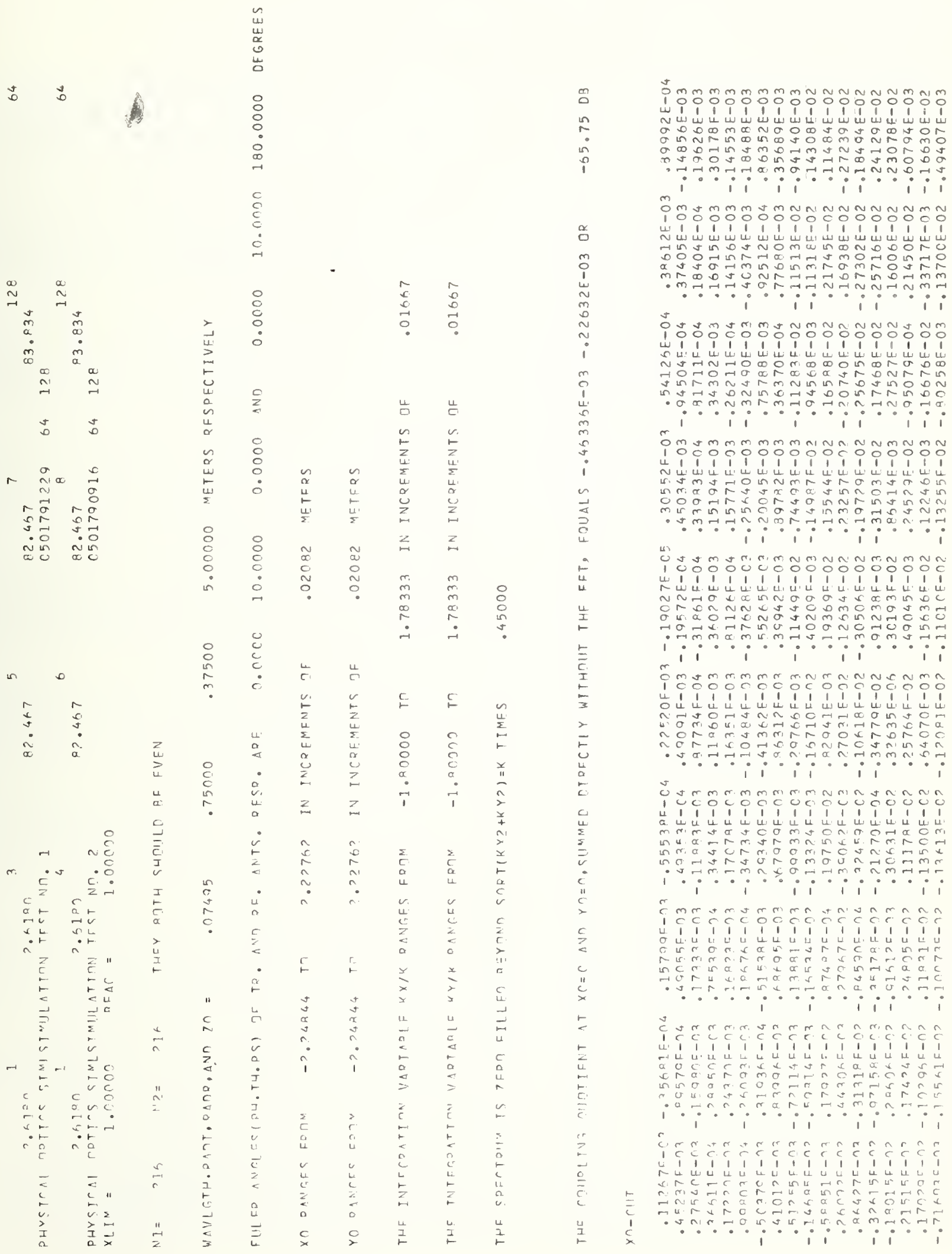


mmmmmámmmmmm

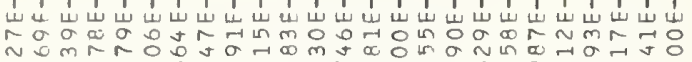

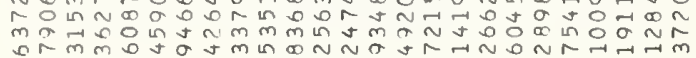

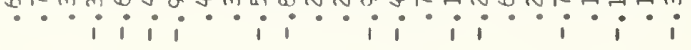

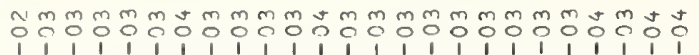

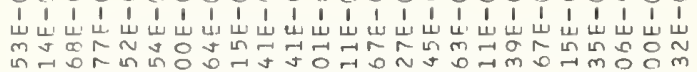

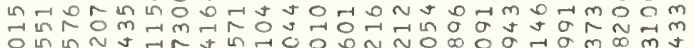

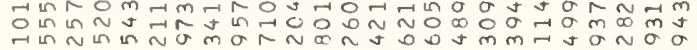

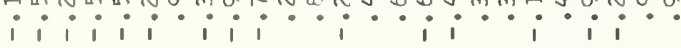

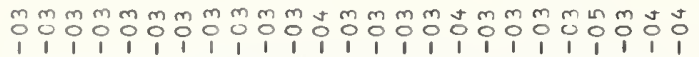

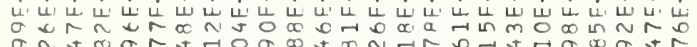

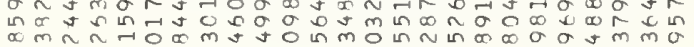

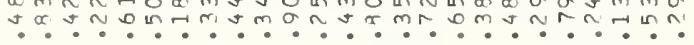

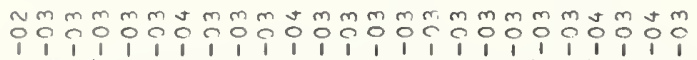

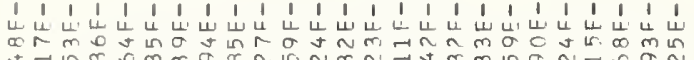

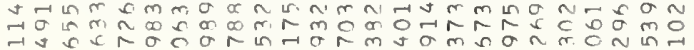

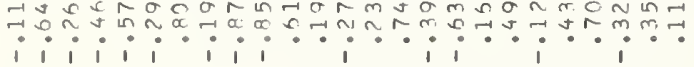
mámómmmmmmmóm

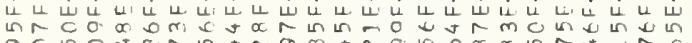

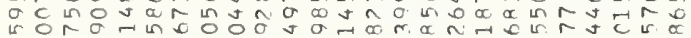

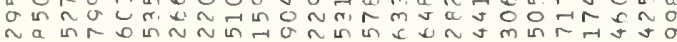

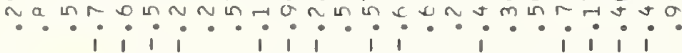

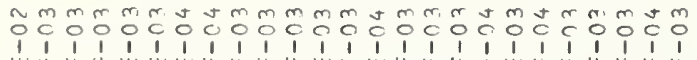

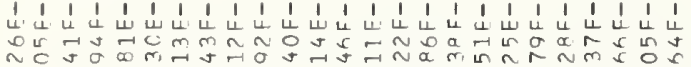
0
0

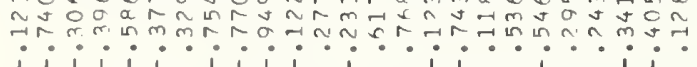

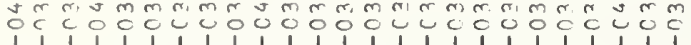

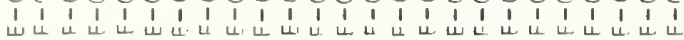

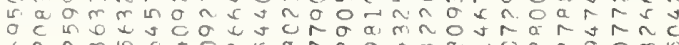

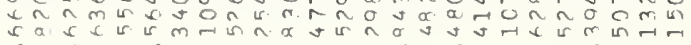

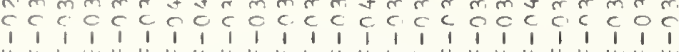

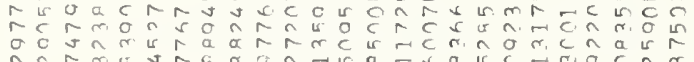

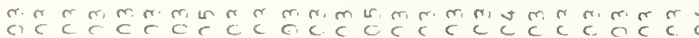

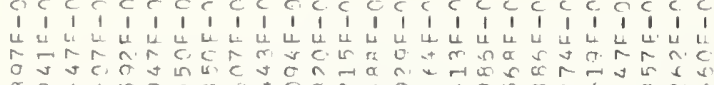

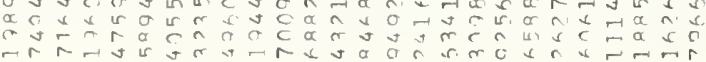

दै

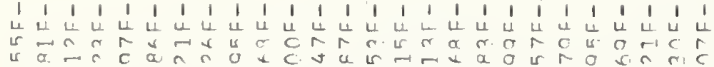

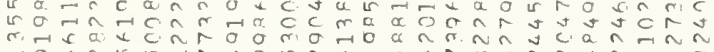

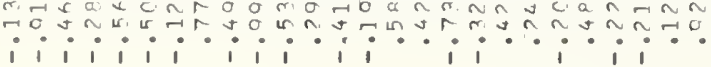

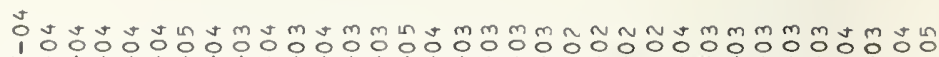

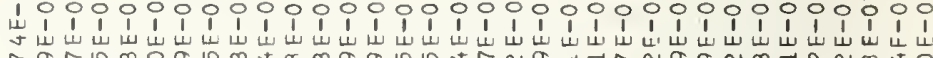

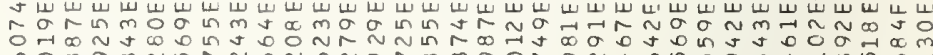

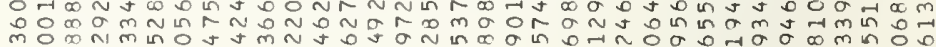

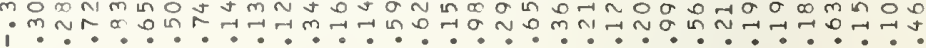

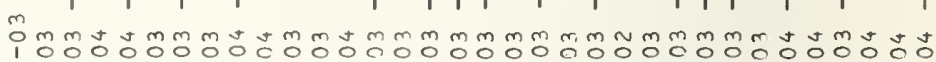

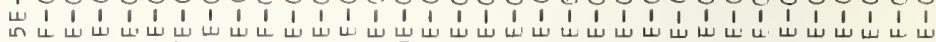

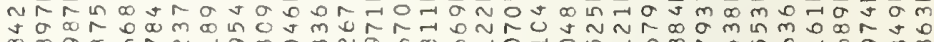

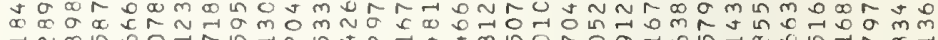

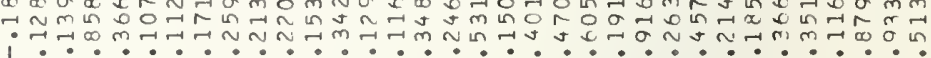
$1 \quad i i^{\prime} i_{i} \cdots i_{i} i_{i} i_{i} \cdots i_{i}$

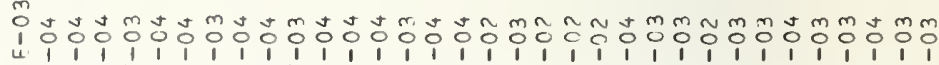

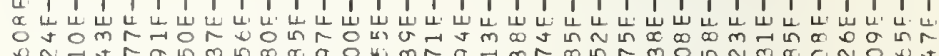

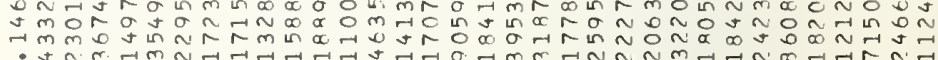

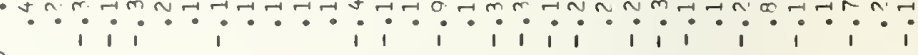

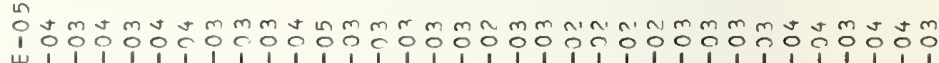

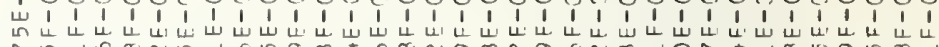

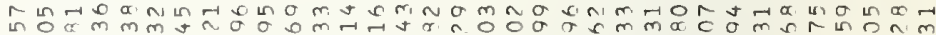

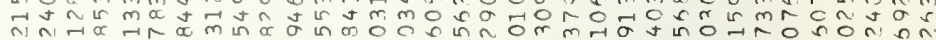

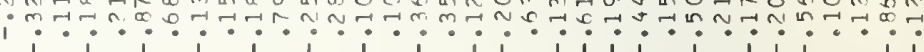

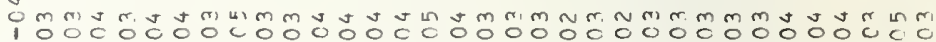

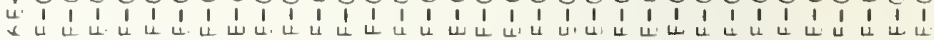

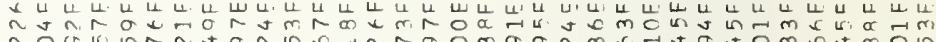

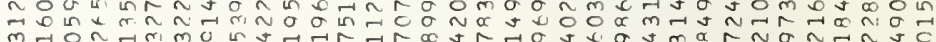
m.

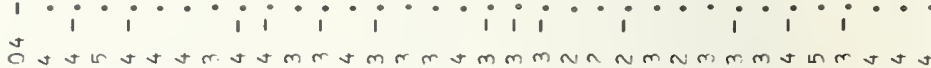

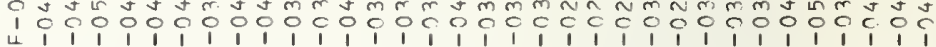

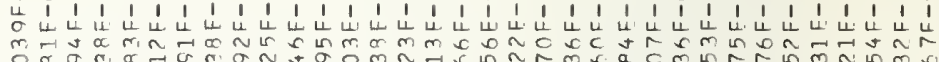

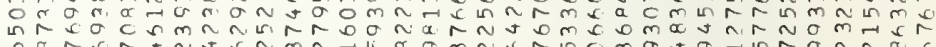

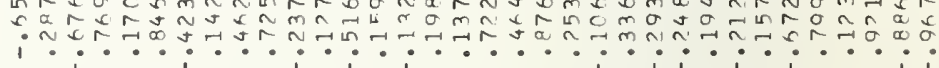

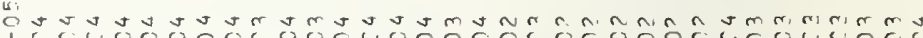

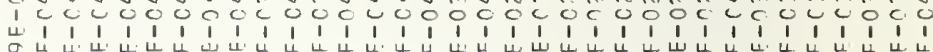

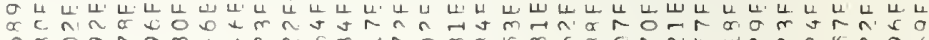

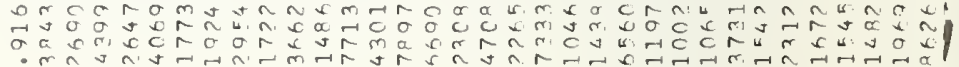

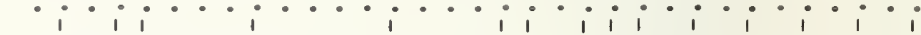

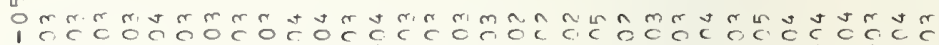

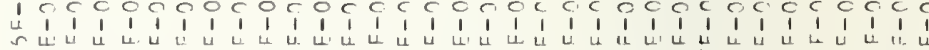

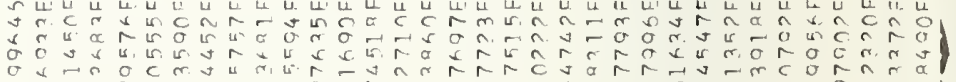
0
$i$

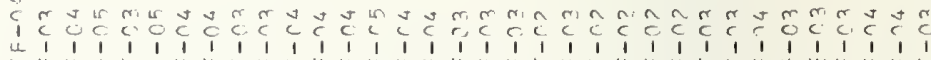

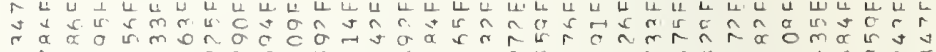

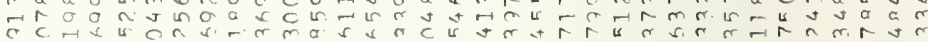

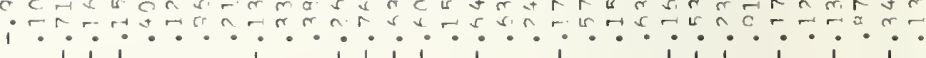

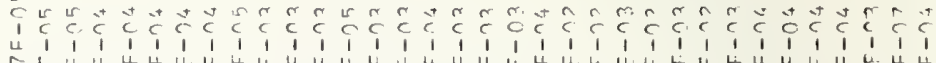

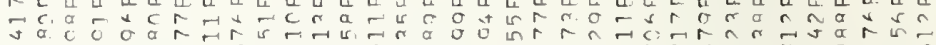

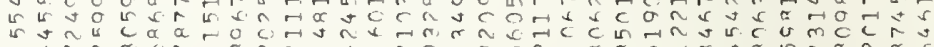

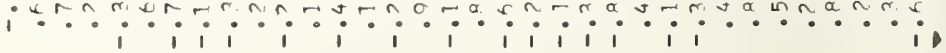




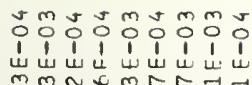

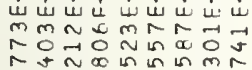

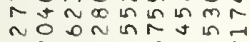

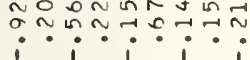
mo

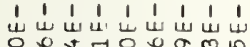

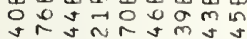
na natoma 떼 - - . i i i

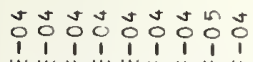

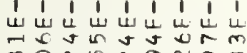
mon $00 \approx 0 m$

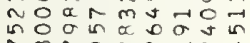
ํำ?

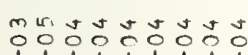

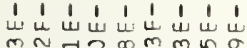

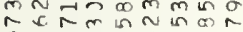
on $\rightarrow$ n

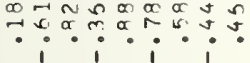

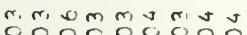

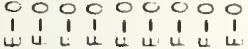
$m \sim \sigma a m s N \approx \sigma$ $m u \sigma a \alpha m m \tilde{r}$

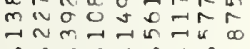
$\because \because \frac{\pi}{1} \because \cdots$

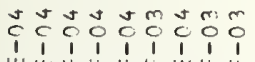

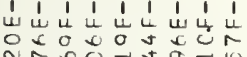

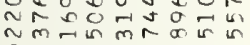

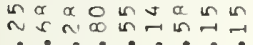
- i c i i i

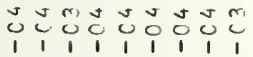

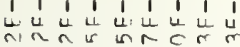

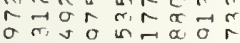

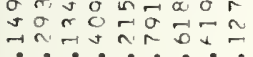
i?

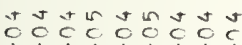

$\begin{array}{lllllll}1 & 1 & 1 & 1\end{array}$

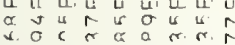

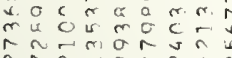

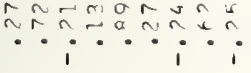

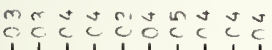

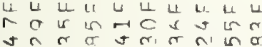

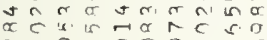

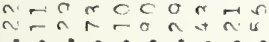

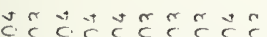
\begin{tabular}{llllllllll}
1 & 1 & 1 & 1 & 1 & 1 & 1 & 1 & 1 & 1 \\
\hline & 1 & 4 & 4 & 4 & 11 & 4 & 4 & 5 & $u$
\end{tabular}

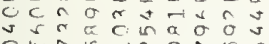
r. $i \because 0$ ㄴ

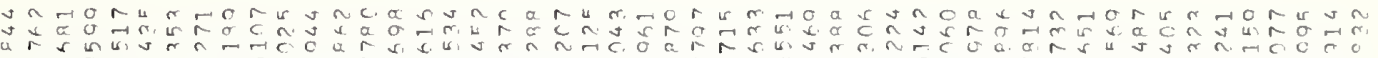

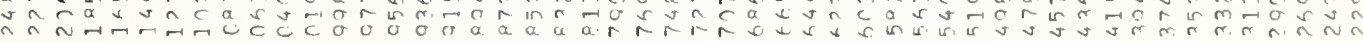

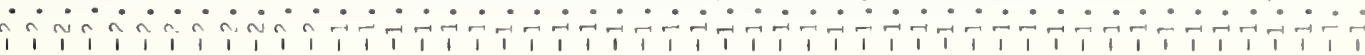

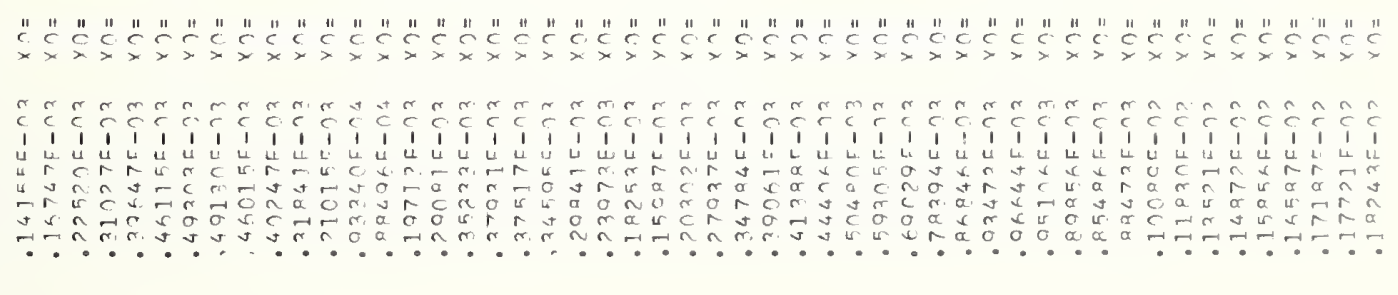




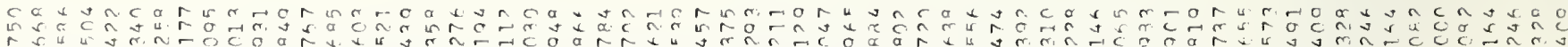

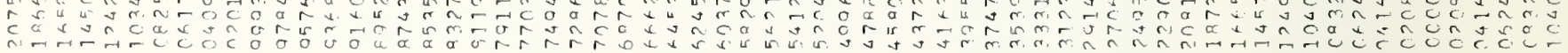

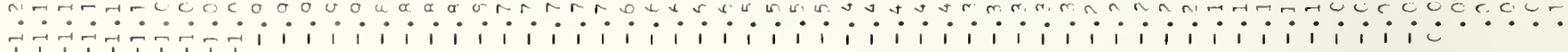

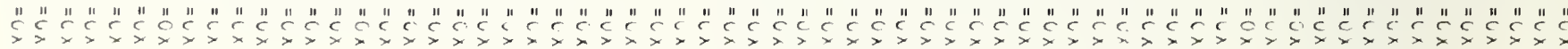

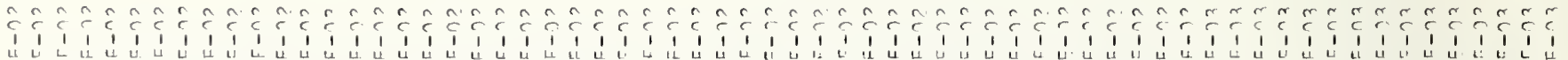
accos

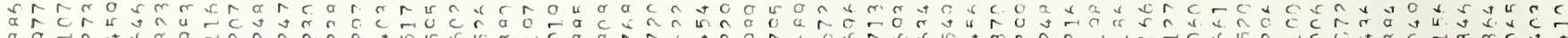
a 


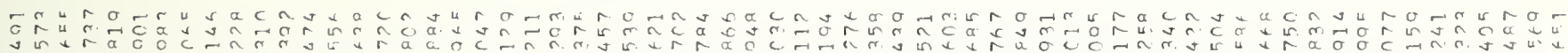

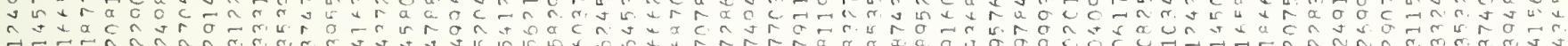

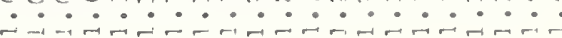

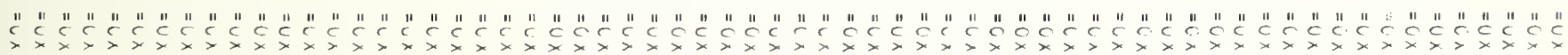

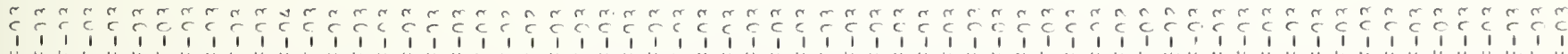
-

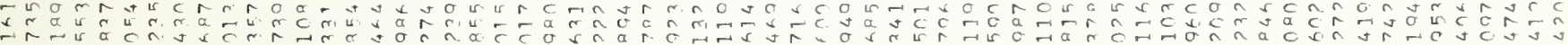

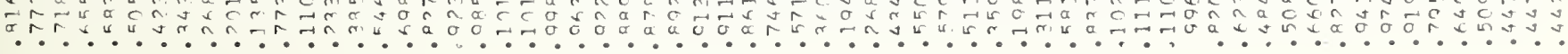




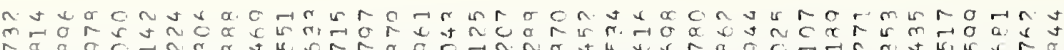

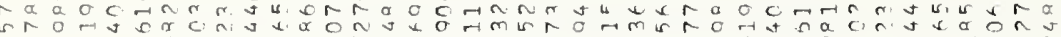
v。

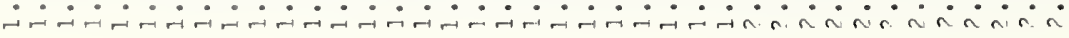

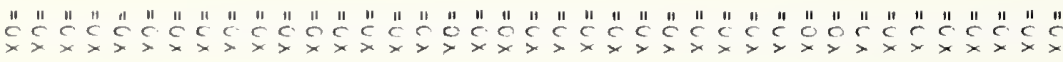

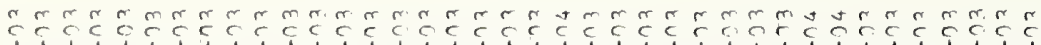

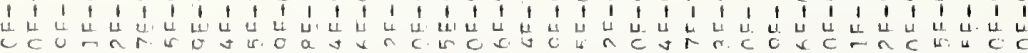

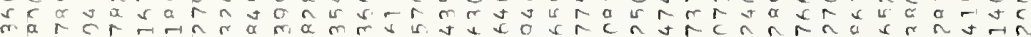

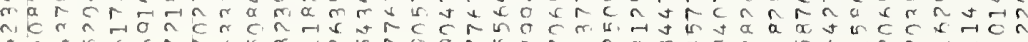

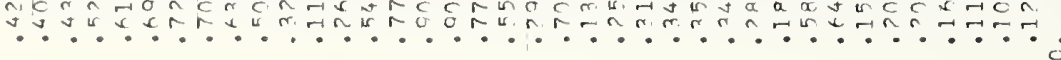




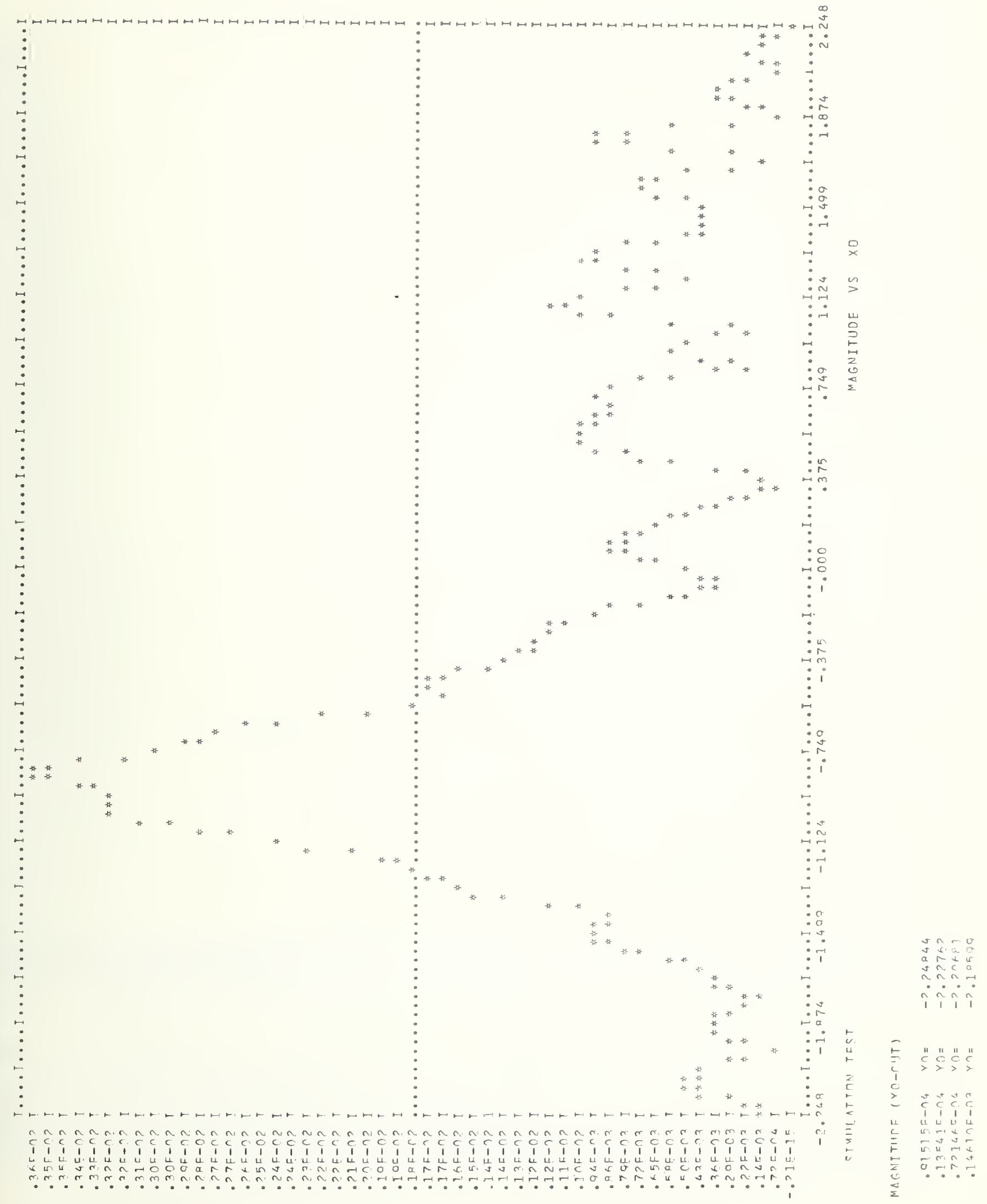




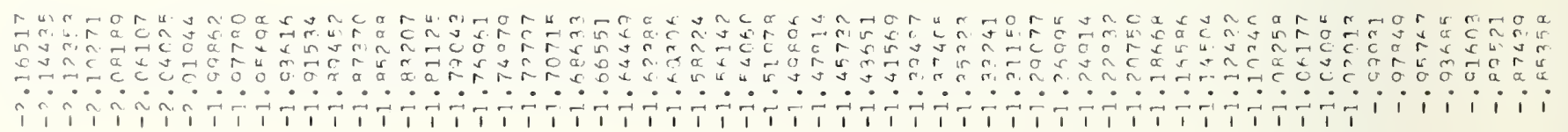

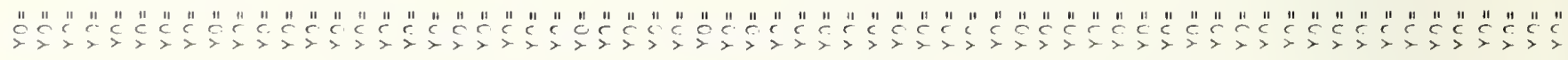

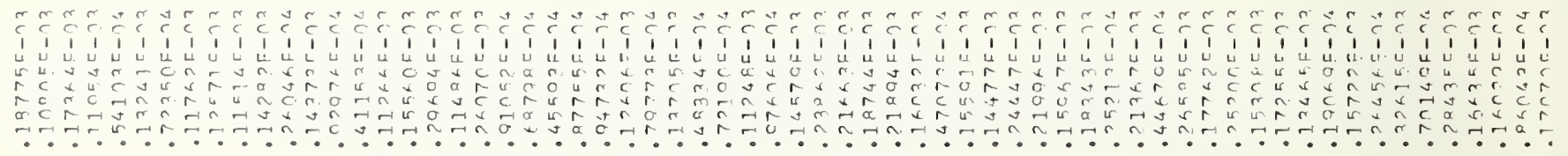




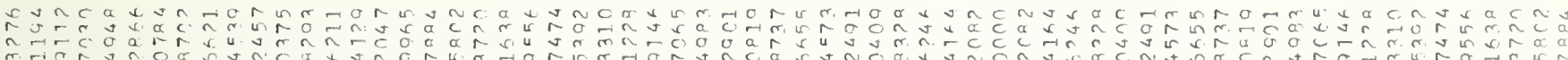
等 a . . . . . . .

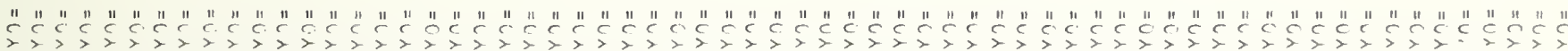

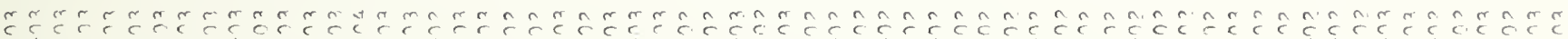

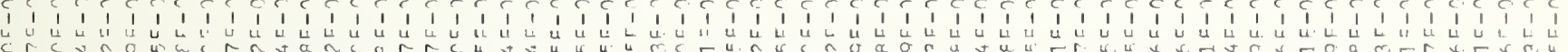

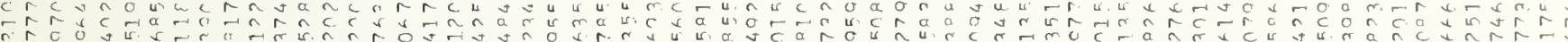

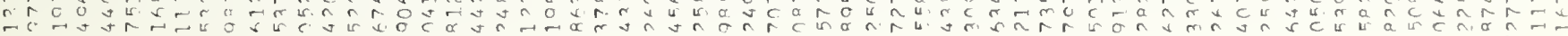

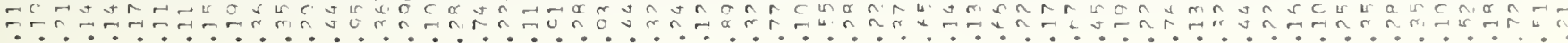




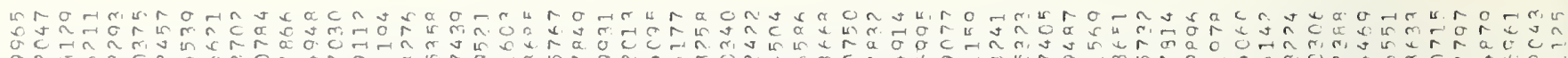

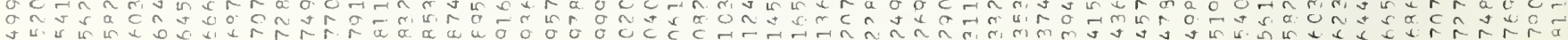

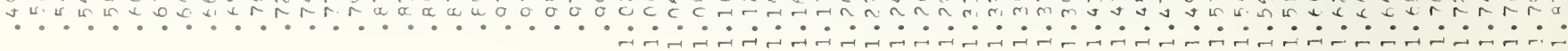

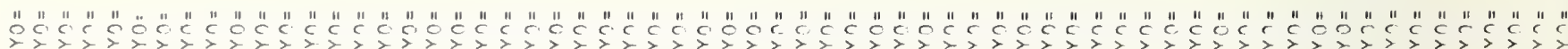

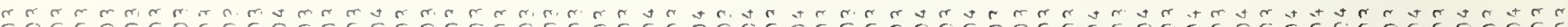
IF TFF

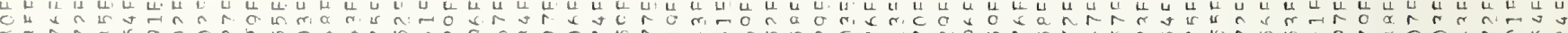
F.

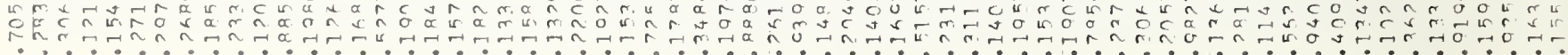




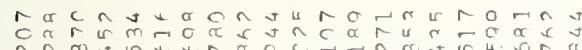

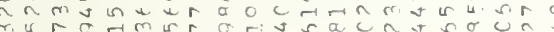

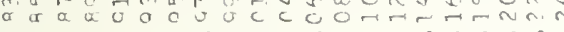

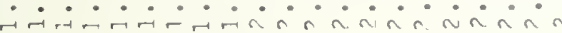

¿゙

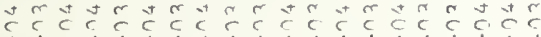

$\begin{array}{lllllllllllllllllll}1 & 1 & 1 & 1 & 1 & 1 & 1 & 1 & 1 & 1 & 1 & 1 & 1 & 1 & 1 & 1 & 1 & 1 & 1\end{array}$

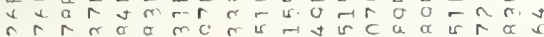

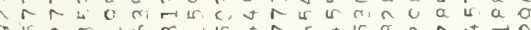

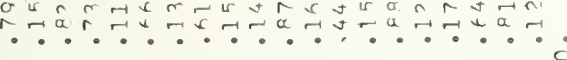




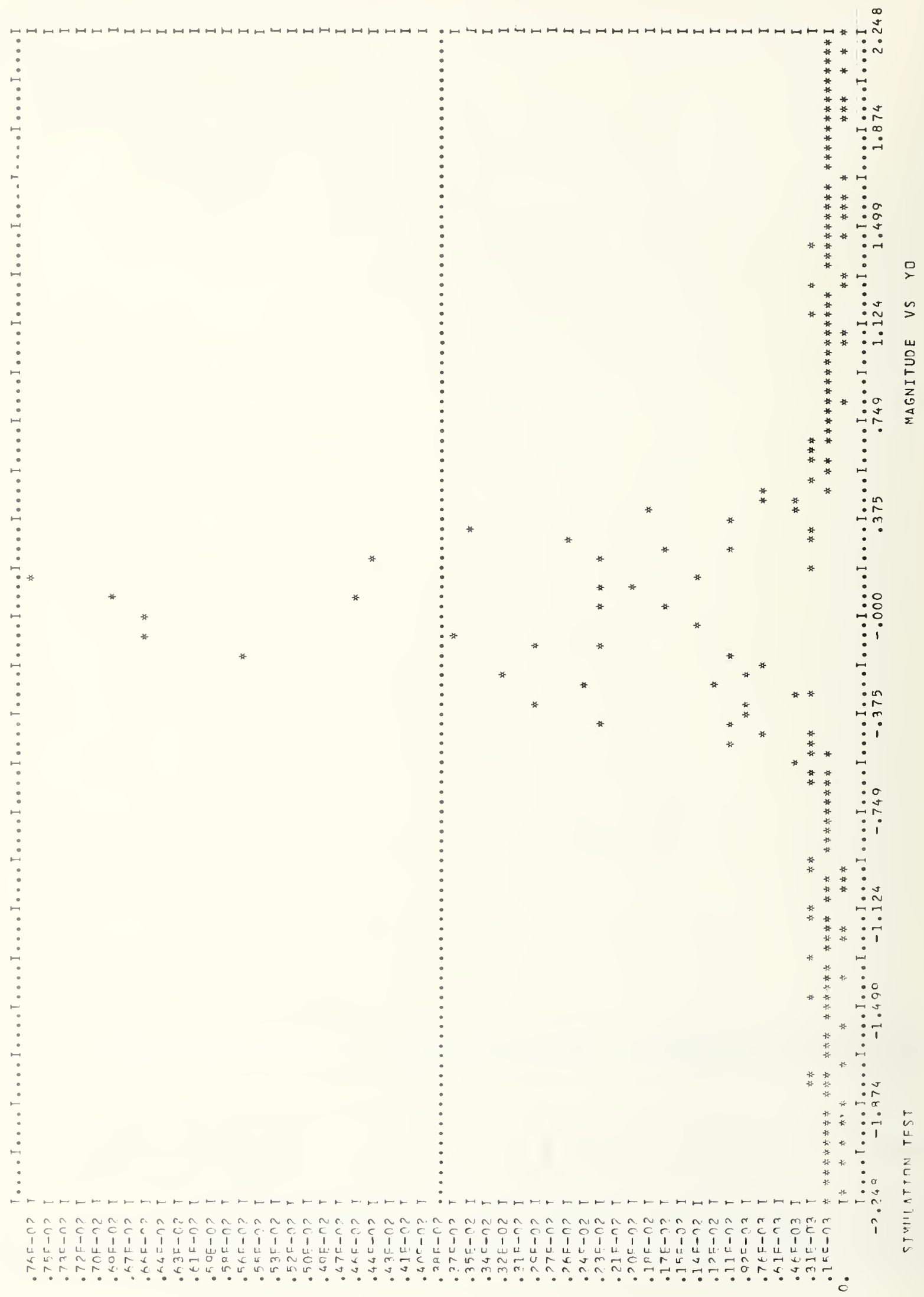


NBS-114A (REV. 2-8C)

U.S. DEPT. OF COMM.

1. PUBLICATION OR

REPORT NO.

NBSIR $80-1630$

2. Performing Organ. Report No.

3. Publication Date

BIBLIOGRAPHIC DATA

SHEET (See instructions)

June 1981

4. TITLE AND SUBTITLE

DETERMINATION OF MUTUAL COUPLING BETWEEN CO-SITED MICROWAVE ANTENNAS AND

CALCULATION OF NEAR-ZONE ELECTRIC FIELD

5. AUTHOR(S)

C. F. Stubenrauch and A. D. Yaghjian

6. PERFORMING ORGANIZATION (If joint or other than NBS, see instructions)

NATIONAL BUREAU OF STANDARDS

DEPARTMENT OF COMMERCE

WASHINGTON, D.C. 20234

. Contract/Grant No.

DAEA-76-F-D760

8. Type of Report \& Period Covered NBSIR

JuTy 1976 - June 1978

9. SPONSORING ORGANIZATION NAME AND COMPLETE ADDRESS (Street, City. State, ZIP)

US Army Communications Electronics Engineering

Installation Agency

Fort Huachuca, Arizona 85613

10. SUPPLEMENTARY NOTES

Document describes a computer program; SF-185, FIPS Software Summary, is attached.

11. ABSTRACT (A 200-word or less factual summary of most significant information. If document includes a significant bibliography or literature survey. mention it here)

The theory and computer programs which allow the efficient computation of coupling between co-sited antennas given their far-field patterns are developed. Coupling between two paraboloidal reflector antennas is computed using both measured far-field patterns and far-field patterns which were obtained from a physical optics (PO) model. These computed results are then compared to the coupling measured directly on an outdoor antenna range. Far fields calculated using the PO model are compared to those obtained from transformed near-field measurements for several reflector antennas. Theory and algorithms are also developed for calculating near-field patterns from far fields obtained from the PO model. Documentation of the near-field and coupling computer programs is presented in the appendices. Conclusions and recommendations for future work are included.

12. KEY WORDS (Six to twelve entries; alphabetical order; capitalize only proper names; and separate key words by semicolons) Co-sited antennas; coupling; far fields; near fields; physical optics; planewave spectrum; reflector antennas.

13. AVAILABILITY

$X \backslash$ Unlimited

For Official Distribution. Do Not Release to NTIS

Order From Superintendent of Documents, U.S. Government Printing Office, Washington, D.C. 20402.

X Order From National Technical Information Service (NTIS), Springfield, VA. 2216I
14. NO. OF

PRINTED PAGES

176

15. Price

$\$ 12.00$ 



